\title{
Hitchin characters and geodesic laminations
}

\author{
by
}

\section{FRANCIS BONAHON}

University of Southern California

Los Angeles, CA, U.S.A.

\author{
Guillaume Dreyer \\ University of Notre Dame \\ Notre Dame, IN, U.S.A. \\ and \\ University of Southern California \\ Los Angeles, CA, U.S.A.
}

\section{Contents}

Introduction . . . . . . . . . . . . . . . . . . . . . . . . . . . . . . . . . . 202

0.1. Background and motivation . . . . . . . . . . . . . . 202

0.2 . Main results . . . . . . . . . . . . . . . . . . 204

1. Generic configurations of flags . . . . . . . . . . . . . . . . . . 209

1.1. Flags . . . . . . . . . . . . . . . . . . . . . 209

1.2. Wedge-product invariants of generic flag triples . . . . . . . . . . . . . . . . . . . . . . . 210

1.3. Quadruple ratios . . . . . . . . . . . . . . . . . . . . 212

1.4. Double ratios . . . . . . . . . . . . . . . . . . . 213

1.5. Positivity . . . . . . . . . . . . . . . . . . . . . . . 214

2. Geodesic laminations . . . . . . . . . . . . . . . . . . . . 214

3. Triangle invariants . . . . . . . . . . . . . . . . . . . . . . . . . . . . . . . . 216

3.1. The flag curve . . . . . . . . . . . . . . . . . . 216

3.2. Triangle invariants of Hitchin characters . . . . . . . . . . 218

4. Tangent cycles for a geodesic lamination . . . . . . . . . . . . 219

4.1. Tangent cycles . . . . . . . . . . . . . . . . . . . . . . 219

4.2. Train-track neighborhoods . . . . . . . . . . . . . 220

4.3. Homological interpretation of tangent cycles . . . . . . . . . . . . 223

4.4. Tangent cycles relative to the slits . . . . . . . . . . 224

4.5. Homological interpretation of tangent cycles relative to the slits 226

4.6. Twisted relative tangent cycles . . . . . . . . . . . . . 229

4.7. Relative tangent cycles from another viewpoint . . . . . . . 231

This research was partially supported by the grants DMS-0604866, DMS-1105402 and DMS1406559 from the U.S. National Science Foundation, and by a Fellowship from the Simons Foundation (grant 301050). In addition, the authors gratefully acknowledge support from the NSF grants DMS-1107452, 1107263 and 1107367 "RNMS: GEometric structures And Representation varieties" (the GEAR Network). 
5. The shearing tangent cycle of a Hitchin character . . . . . . . . . . 234

5.1. Slithering . . . . . . . . . . . . . . . . . . . 234

5.2. The shearing cycle . . . . . . . . . . . . . . . . . . . 244

6. Hitchin characters are determined by their invariants . . . . . . . . 251

6.1. Revisiting the slithering map . . . . . . . . . . . . . 251

6.2. Reconstructing a Hitchin homomorphism from its invariants . 252

7. Length functions . . . . . . . . . . . . . . . . . . 258

7.1. Length functions associated with Hitchin characters . . . . . 258

7.2. Shearing cycles and length functions . . . . . . . . . . . 261

8. Parametrizing Hitchin components . . . . . . . . . . . . . . . . . . 272

8.1. Constraints between invariants . . . . . . . . . . . 273

8.2. An estimate from the positive intersection condition . . . . . 279

8.3. Realization of invariants, and parametrization of $\operatorname{Hit}_{n}(S)$. . 283

8.4. Constraints among triangle invariants, and on shearing cycles 286

9. The action of pseudo-Anosov homeomorphisms on the Hitchin com-

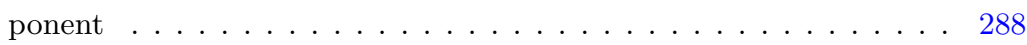

10. Length functions of measured laminations . . . . . . . . . . . 290

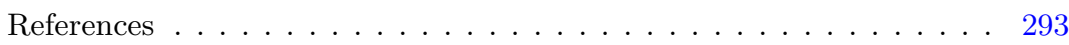

\section{Introduction}

\subsection{Background and motivation}

For a closed, connected, oriented surface $S$ of genus $g>1$, the Hitchin component $\operatorname{Hit}_{n}(S)$ is a preferred component of the character variety

$$
X_{\mathrm{PSL}_{n}(\mathbb{R})}(S)=\left\{\text { homomorphisms } \varrho: \pi_{1}(S) \rightarrow \mathrm{PSL}_{n}(\mathbb{R})\right\} / / \mathrm{PSL}_{n}(\mathbb{R})
$$

consisting of group homomorphisms $\varrho: \pi_{1}(S) \rightarrow \mathrm{PSL}_{n}(\mathbb{R})$ from the fundamental group $\pi_{1}(S)$ to the Lie group $\mathrm{PSL}_{n}(\mathbb{R})$ (equal to the special linear group $\mathrm{SL}_{n}(\mathbb{R})$ if $n$ is odd, and to $\mathrm{SL}_{n}(\mathbb{R}) /\{ \pm \mathrm{Id}\}$ if $n$ is even), where $\operatorname{PSL}_{n}(\mathbb{R})$ acts on these homomorphisms by conjugation. The quotient should normally be taken in the sense of geometric invariant theory [34], but this subtlety is irrelevant here, as this quotient construction coincides with the usual topological quotient on the Hitchin component. The "character variety" terminology is justified by the fact that, by [37], the class of $\varrho: \pi_{1}(S) \rightarrow \mathrm{PSL}_{n}(\mathbb{R})$ in $\chi_{\mathrm{PSL}_{n}(\mathbb{R})}(S)$ is determined by the character $\pi_{1}(S) \rightarrow \mathbb{R}$ associating the trace $\operatorname{Tr} \varrho(\gamma) \in \mathbb{R}$ with $\gamma \in \pi_{1}(S)$ (defined up to sign when $n$ is even). In particular, we will refer to the elements of $\chi_{\mathrm{PSL}_{n}(\mathbb{R})}(S)$ as characters.

When $n=2$, the Lie group $\mathrm{PSL}_{2}(\mathbb{R})$ is also the orientation-preserving isometry group of the hyperbolic plane $\mathbb{H}^{2}$, and the Hitchin component $\operatorname{Hit}_{2}(S)$ of $\mathcal{X}_{\mathrm{PSL}_{2}(\mathbb{R})}(S)$ consists 
of all characters represented by injective homomorphisms $\varrho: \pi_{1}(S) \rightarrow \mathrm{PSL}_{2}(\mathbb{R})$ whose image $\varrho\left(\pi_{1}(S)\right)$ is discrete in $\mathrm{PSL}_{2}(\mathbb{R})$ and for which the natural homotopy equivalence $S \rightarrow \mathbb{H}^{2} / \varrho\left(\pi_{1}(S)\right)$ has degree 1 . The Hitchin component $\operatorname{Hit}_{2}(S)$ is in this case called the Teichmüller component, and can also be described as the space of isotopy classes of hyperbolic metrics on $S$.

When $n>2$, there is a preferred homomorphism $\mathrm{PSL}_{2}(\mathbb{R}) \rightarrow \mathrm{PSL}_{n}(\mathbb{R})$ coming from the unique $n$-dimensional representation of $\mathrm{SL}_{2}(\mathbb{R})$ (or, equivalently, from the natural action of $\mathrm{SL}_{2}(\mathbb{R})$ on the vector space $\mathbb{R}[X, Y]_{n-1} \cong \mathbb{R}^{n}$ of homogeneous polynomials of degree $n-1$ in two variables). This provides a natural map $\mathcal{X}_{\mathrm{PSL}_{2}(\mathbb{R})}(S) \rightarrow \mathcal{X}_{\mathrm{PSL}_{n}(\mathbb{R})}(S)$, and the Hitchin component $\operatorname{Hit}_{n}(S)$ is the component of $\chi_{\mathrm{PSL}_{n}(\mathbb{R})}(S)$ that contains the image of $\operatorname{Hit}_{2}(S) \subset \mathcal{X}_{\mathrm{PSL}_{2}(\mathbb{R})}(S)$. The terminology is motivated by the following fundamental result by Hitchin [23], who was the first to single out this component.

THEOREM 0.1. (Hitchin) The Hitchin component $\operatorname{Hit}_{n}(S)$ is diffeomorphic to

$$
\mathbb{R}^{2(g-1)\left(n^{2}-1\right)} .
$$

A Hitchin character is an element of the Hitchin component $\operatorname{Hit}_{n}(S)$, and a Hitchin homomorphism is a homomorphism $\varrho: \pi_{1}(S) \rightarrow \mathrm{PSL}_{n}(\mathbb{R})$ representing a Hitchin character. We will use the same letter to represent the Hitchin homomorphism $\varrho: \pi_{1}(S) \rightarrow \mathrm{PSL}_{n}(\mathbb{R})$ and the corresponding Hitchin character $\varrho \in \operatorname{Hit}_{n}(S)$.

About 15 years after [23], Labourie [27] showed that Hitchin homomorphisms satisfy many important geometric and dynamical properties, and in particular are injective with discrete image; see also [18].

Hitchin's construction of the parametrization of $\operatorname{Hit}_{n}(S)$ given by Theorem 0.1 is based on geometric analysis techniques that provide little information on the geometry of the Hitchin homomorphisms themselves; see [30], [28], [29] for different geometric analytic parametrizations when $n=3$. The current article is devoted to developing another parametrization of the Hitchin component $\operatorname{Hit}_{n}(S)$ which is much more geometric, and has the additional advantage of being well-behaved with respect to the data of a geodesic lamination. Geodesic laminations were introduced by Thurston in order to develop a continuous calculus for simple closed curves on the surface $S$, and provide very powerful tools for many topological and geometric problems in dimensions 2 and 3 . See $\S 9$ and $\S 10$ for two simple applications of our parametrization, one to the dynamics of the action of a pseudo-Anosov homomorphism of $S$ on the Hitchin component, and another one to the length functions defined by a Hitchin character on Thurston's space $\mathcal{M} \mathcal{L}(S)$ of measured laminations on $S$.

In fact, our construction is a natural extension of Thurston's parametrization of the Teichmüller component by shearing coordinates [42], [5]. It draws its inspiration from 
this classical case where $n=2$, but also from work of Fock-Goncharov [18] on a variant of the Hitchin component where the surface $S$ has punctures, and where these punctures are endowed with additional information. As in the classical case where $n=2$, the situation is conceptually and analytically much more complicated for a closed surface than in the case considered in [18]. The major contributions of the article are to adapt the analysis of [42], [5] to the case where $n \geqslant 2$. As a consequence, many of the arguments are new even for the case $n=2$ : this includes the slithering map of $\S 5.1$, the explicit reconstruction of a character from its shearing invariants in $\S 6.2$, and the estimates of $\S 8.2$ relating growth rates to positivity.

The companion article [9] is devoted to a special case of our parametrization, when the geodesic lamination has only finitely many leaves. The situation is much simpler in that case, and in particular the arguments of [9] tend to be very combinatorial in nature. The current article has a much more analytic flavor. It is also more conceptual, and provides a homological interpretation of some of the invariants and phenomena that were developed in a purely computational way in [9]. And of course the framework of general geodesic laminations, possibly with uncountably many leaves, considered in this article is better suited for applications.

The article [16] was developed, to a large extent, as a first step towards the more general results of the current paper. It investigates all deformations of a Hitchin character $\varrho \in \operatorname{Hit}_{n}(S)$ that respect its triangle invariants, as discussed in the next section.

\subsection{Main results}

We can now be more specific. Let $\lambda$ be a maximal geodesic lamination in $S$. See $\S 2$ for precise definitions. What we need to know here is just that, for an arbitrary auxiliary metric of negative curvature on the surface, $\lambda$ is decomposed as a union of disjoint geodesic leaves, and that its complement $S \backslash \lambda$ consists of $4(g-1)$ infinite triangles with geodesic boundary. Some maximal geodesic laminations, such as the ones considered in [9], have only a finite number of leaves, but generic examples have uncountably many leaves.

Given a Hitchin character $\varrho \in \operatorname{Hit}_{n}(S)$, the rich dynamical structure for $\varrho$ discovered by Labourie [27] associates with each triangle component $T_{j}$ of $S \backslash \lambda$ a triple $(E, F, G)$ of three flags of $\mathbb{R}^{n}$, well defined modulo the action of $\mathrm{PGL}_{n}(\mathbb{R})$. In addition, Fock and Goncharov [18] prove that this flag triple $(E, F, G)$ is positive, in a sense discussed in $\S 1.5$, and is determined by $\frac{1}{2}(n-1)(n-2)$ invariants $\tau_{a b c}^{\varrho}(E, F, G) \in \mathbb{R}$. Since $S \backslash \lambda$ has $4(g-1)$ components, these flag triple invariants can be collected into a single triangle invariant $\tau^{\varrho} \in \mathbb{R}^{2(g-1)(n-1)(n-2)}$. 
The really new feature introduced in this article describes how to glue these flag triples across the (possibly uncountably many) leaves of the lamination, and simultaneously involves analytic and combinatorial arguments. The analytic part of this analysis is based on the slithering map constructed in $\S 5.1$, which is a higher-dimensional analogue of the horocyclic foliation that is at the basis of the case $n=2$ [42], [5]. This slithering map enables us to control the gluing by elements of the homology of a train-track neighborhood $U$ for $\lambda$, which we now briefly describe. The precise definition of train-track neighborhoods can be found in $\S 4.2$ (and is familiar to experts); at this point, it suffices to say that $U$ is obtained from $S$ by removing $4(g-1)$ disjoint disks, one in each component of $S \backslash \lambda$. In addition, the boundary $\partial U$ is decomposed into a horizontal boundary $\partial_{\mathrm{h}} U$ and a vertical boundary $\partial_{\mathrm{v}} U$ in such a way that, for each component $T$ of $S \backslash \lambda$, the intersection $T \cap \partial U$ is a hexagon made up of three arc components of $\partial_{\mathrm{h}} U$ running parallel to the sides of $T$ and three arc components of $\partial_{\mathrm{v}} U$ facing the spikes of $T$.

The geodesic lamination $\lambda$ has a well-defined 2-fold orientation cover $\hat{\lambda}$, whose leaves are continuously oriented, and the covering map $\hat{\lambda} \rightarrow \lambda$ uniquely extends to a 2 -fold cover $\widehat{U} \rightarrow U$. In particular, $\hat{\lambda}$ is a geodesic lamination in the surface $\widehat{U}$. Also, as the components of $S \backslash \lambda$ are infinite triangles, $\lambda$ is non-orientable and it follows that $\hat{\lambda}$ and $\widehat{U}$ are connected.

Our new invariant for a Hitchin character $\varrho \in \operatorname{Hit}_{n}(S)$ is a certain shearing class $\left[\sigma^{\varrho}\right] \in H_{1}\left(\widehat{U}, \partial_{\mathrm{v}} \widehat{U} ; \mathbb{R}^{n-1}\right)$. This shearing class has the property that $\iota_{*}\left(\left[\sigma^{\varrho}\right]\right)=-\overline{\left[\sigma^{\varrho}\right]}$, for the covering involution $\iota$ of the cover $\widehat{U} \rightarrow U$ and for the involution $x \mapsto \bar{x}$ of $\mathbb{R}^{n-1}$ that associates $\bar{x}=\left(x_{n-1}, x_{n-2}, \ldots, x_{1}\right)$ with $x=\left(x_{1}, x_{2}, \ldots, x_{n-1}\right)$. In particular, $\left[\sigma^{\varrho}\right]$ can also be interpreted as a twisted homology class $\left[\sigma^{\varrho}\right] \in H_{1}\left(U, \partial_{\mathrm{v}} U ; \widetilde{\mathbb{R}}^{n-1}\right)$ valued in a suitable coefficient bundle $\widetilde{\mathbb{R}}^{n-1}$ over $U$ with fiber $\mathbb{R}^{n-1}$.

The triangle invariant $\tau^{\varrho}$ and the shearing class $\left[\sigma^{\varrho}\right]$ satisfy two types of constraints. The first constraint is a homological equality.

Proposition 0.2. (Shearing cycle boundary condition) The homological boundary $\partial\left[\sigma^{\varrho}\right] \in H_{0}\left(\partial_{\mathrm{v}} \widehat{U} ; \mathbb{R}^{n-1}\right)$ of the shearing class $\left[\sigma^{\varrho}\right] \in H_{1}\left(\widehat{U}, \partial_{\mathrm{v}} \widehat{U} ; \mathbb{R}^{n-1}\right)$ of a Hitchin character $\varrho \in \operatorname{Hit}_{n}(S)$ is completely determined by the triangle invariant $\tau^{\varrho} \in \mathbb{R}^{2(g-1)(n-1)(n-2)}$, by an explicit linear formula given in Lemma 5.14 and Proposition 5.15.

The second constraint is a positivity property, proved as Corollary 7.13 in $§ 7.2$. Because the leaves of the orientation cover $\hat{\lambda}$ are oriented, a famous construction by Ruelle and Sullivan [38] interprets every transverse measure $\mu$ for $\hat{\lambda}$ as a 1-dimensional de Rham current in $\widehat{U}$. In particular, such a transverse measure $\mu$ determines a homology class $[\mu] \in H_{1}(\widehat{U} ; \mathbb{R})$. See also Proposition 4.2 for a more explicit description of this homology class $[\mu]$. 
Proposition 0.3. (Positive intersection condition) For every transverse measure $\mu$ for the orientation cover $\hat{\lambda}$, the algebraic intersection vector $[\mu] \cdot\left[\sigma^{\varrho}\right] \in \mathbb{R}^{n-1}$ of the homology class $[\mu] \in H_{1}(\widehat{U} ; \mathbb{R})$ with the shearing class $\left[\sigma^{\varrho}\right] \in H_{1}\left(\widehat{U}, \partial_{\mathrm{v}} \widehat{U} ; \mathbb{R}^{n-1}\right)$ of $\varrho \in \operatorname{Hit}_{n}(S)$ is positive, in the sense that all its coordinates are positive.

The shearing cycle boundary and positive intersection conditions restrict the pair $\left(\tau^{\varrho},\left[\sigma^{\varrho}\right]\right)$ to a convex polyhedral cone $\mathcal{P}$ in $\mathbb{R}^{2(g-1)(n-1)(n-2)} \times H_{1}\left(\widehat{U}, \partial_{\mathrm{v}} \widehat{U} ; \mathbb{R}^{n-1}\right)$, with finitely many faces. The main result of the article, proved as Theorem 8.13 in $\S 8.3$, shows that these are the only restrictions on the triangle and shearing invariants, and that these provide a parametrization of the Hitchin component $\operatorname{Hit}_{n}(S)$.

ThEOREM 0.4. (Parametrization of the Hitchin component) The $\operatorname{map}_{\operatorname{Hit}}(S) \rightarrow \mathcal{P}$, which to a Hitchin character $\varrho \in \operatorname{Hit}_{n}(S)$ assigns the pair $\left(\tau^{\varrho},\left[\sigma^{\varrho}\right]\right)$ formed by its triangle invariant $\tau^{\varrho} \in \mathbb{R}^{2(g-1)(n-1)(n-2)}$ and its shearing class $\left[\sigma^{\varrho}\right] \in H_{1}\left(\widehat{U}, \partial_{\mathrm{v}} \widehat{U} ; \mathbb{R}^{n-1}\right)$, is a homeomorphism.

When $n=2$, all triangle invariants are trivial and the shearing class $\left[\sigma^{\varrho}\right]$ is closed. The above theorem is just a rephrasing of the parametrization of the Teichmüller space $\mathcal{T}(S)$ by the shearing coordinates defined by $\lambda$, as developed in [42] and [5].

The shearing cycle boundary condition provides some unexpected constraints on the triangle invariants of Hitchin characters, as well as on their shearing classes. The following two statements are abbreviated expressions of more specific computations given in $\S 8.4$. These restrictions are somewhat surprising when one considers the relatively large dimension $2(g-1)\left(n^{2}-1\right)$ of $\operatorname{Hit}_{n}(S)$.

Proposition 0.5. An element $\tau \in \mathbb{R}^{2(g-1)(n-1)(n-2)}$ is the triangle invariant $\tau^{\varrho}$ of a Hitchin character $\varrho \in \operatorname{Hit}_{n}(S)$ if and only if it belongs to a certain explicit subspace of codimension $\left\lfloor\frac{1}{2}(n-1)\right\rfloor$ of $\mathbb{R}^{2(g-1)(n-1)(n-2)}$.

We already saw that shearing cycles have a simple symmetry property under the covering involution $\iota$ of $\widehat{U}$, and therefore they belong to the twisted homology space $H_{1}\left(U, \partial_{\mathrm{v}} U ; \widetilde{\mathbb{R}}^{n-1}\right)$, consisting of those $\alpha \in H_{1}\left(\widehat{U}, \partial_{\mathrm{v}} \widehat{U} ; \mathbb{R}^{n-1}\right)$ such that $\iota_{*}(\alpha)=-\bar{\alpha}$. The dimension of this twisted homology space $H_{1}\left(U, \partial_{\mathrm{v}} U ; \widetilde{\mathbb{R}}^{n-1}\right)$ is computed in (the proof of) Proposition 4.7, and is equal to $18(g-1)(n-1)$.

Proposition 0.6. A twisted relative homology class $[\sigma] \in H_{1}\left(U, \partial_{\mathrm{v}} U ; \widetilde{\mathbb{R}}^{n-1}\right)$ is the shearing class $\left[\sigma^{\varrho}\right]$ of a Hitchin character $\varrho \in \operatorname{Hit}_{n}(S)$ if and only if it belongs to a certain open convex polyhedral cone, bounded by finitely many faces, in an explicit linear subspace of $H_{1}\left(U, \partial_{\mathrm{v}} U ; \widetilde{\mathbb{R}}^{n-1}\right)$ of codimension $24(g-1)$ if $n>3$, of codimension $20(g-1)$ if $n=3$, and of codimension $12(g-1)$ if $n=2$. 
At first, the relative homology space $H_{1}\left(\widehat{U}, \partial_{\mathrm{v}} \widehat{U} ; \mathbb{R}^{n-1}\right)$ of a train-track neighborhood $U$, and its twisted version $H_{1}\left(U, \partial_{\mathrm{v}} U ; \widetilde{\mathbb{R}}^{n-1}\right)$, may not appear very natural. In fact, although we decided to privilege this more familiar point of view in this introduction, $H_{1}\left(\widehat{U}, \partial_{\mathrm{v}} \widehat{U} ; \mathbb{R}^{n-1}\right)$ occurs as a space $Z\left(\hat{\lambda}\right.$, slits; $\left.\mathbb{R}^{n-1}\right)$ of tangent cycles for the orientation cover $\hat{\lambda}$ relative to its slits, where the slits of $\hat{\lambda}$ are lifts of the spikes of the complement $S \backslash \lambda$; Proposition 4.5 then provides an isomorphism

$$
z\left(\hat{\lambda}, \text { slits } ; \mathbb{R}^{n-1}\right) \cong H_{1}\left(\widehat{U}, \partial_{\mathrm{v}} \widehat{U} ; \mathbb{R}^{n-1}\right)
$$

A relative tangent cycle $\alpha \in \mathcal{Z}\left(\hat{\lambda}\right.$, slits; $\left.\mathbb{R}^{n-1}\right)$ assigns a vector $\alpha(k) \in \mathbb{R}^{n-1}$ to each arc $k$ transverse to $\hat{\lambda}$, in a quasi-additive way: If $k$ is split into two subarcs $k_{1}$ and $k_{2}$, then $\alpha(k)$ is equal to the sum of $\alpha\left(k_{1}\right), \alpha\left(k_{2}\right)$ and of a correction factor depending on the slit of $\hat{\lambda}$ facing the point $k_{1} \cap k_{2}$ along which $k$ was split. In particular, $z\left(\hat{\lambda}\right.$, slits; $\left.\mathbb{R}^{n-1}\right)$ depends only on the maximal geodesic lamination $\lambda$, and not on the train-track neighborhood $U$. For the above isomorphism, the twisted homology space

$$
H_{1}\left(U, \partial_{\mathrm{v}} U ; \widetilde{\mathbb{R}}^{n-1}\right) \subset H_{1}\left(\widehat{U}, \partial_{\mathrm{v}} \widehat{U} ; \mathbb{R}^{n-1}\right)
$$

corresponds to those $\alpha \in Z\left(\hat{\lambda}\right.$, slits; $\left.\mathbb{R}^{n-1}\right)$ such that $\alpha(\iota(k))=\overline{\alpha(k)}$ for every arc $k$ transverse to $\hat{\lambda}$, and for the covering involution $\iota$; in particular, $H_{1}\left(U, \partial_{\mathrm{v}} U ; \widetilde{\mathbb{R}}^{n-1}\right)$ is also independent of the choice of $U$.

The lack of additivity of a relative tangent cycle $\alpha \in Z\left(\hat{\lambda}\right.$, slits; $\left.\mathbb{R}^{n-1}\right)$ has a nice expression in terms of the boundary map $\partial: H_{1}\left(\widehat{U}, \partial_{\mathrm{v}} \widehat{U} ; \mathbb{R}^{n-1}\right) \rightarrow H_{0}\left(\partial_{\mathrm{v}} \widehat{U} ; \mathbb{R}^{n-1}\right)$, and is at the basis of the shearing cycle boundary condition of Proposition 0.2. In the classical case where $n=2$, all triangle invariants are trivial, and the shearing cycle boundary condition says that the shearing class $\left[\sigma^{\varrho}\right] \in H_{1}\left(\widehat{U}, \partial_{\mathrm{v}} \widehat{U} ; \mathbb{R}^{n-1}\right)$ has boundary equal to zero, and in particular that the corresponding tangent cycle $\left[\sigma^{\varrho}\right] \in Z(\hat{\lambda}$, slits; $\mathbb{R})$ is additive with no correction factors; such objects were called "transverse cocycles" in [7] and [5].

This point of view enables us to shed some light on the positive intersection condition of Proposition 0.3. Given a Hitchin character $\varrho \in \operatorname{Hit}_{n}(S)$, Labourie [27] shows that for every non-trivial $\gamma \in \pi_{1}(S)$ the matrix $\varrho(\gamma) \in \operatorname{PSL}_{n}(\mathbb{R})$ is diagonalizable, and that its eigenvalues $m_{a}^{\varrho}(\gamma)$ can be ordered in such a way that $\left|m_{1}^{\varrho}(\gamma)\right|>\left|m_{2}^{\varrho}(\gamma)\right|>\ldots>\left|m_{n}^{\varrho}(\gamma)\right|$. If we define $\ell^{\varrho}(\gamma) \in \mathbb{R}^{n-1}$ by the property that its ath coordinate is

$$
\ell_{a}^{\varrho}(\gamma)=\log \frac{\left|m_{a}^{\varrho}(\gamma)\right|}{\left|m_{a+1}^{\varrho}(\gamma)\right|},
$$

the second author showed in [15] that this formula admits a continuous linear extension $\ell^{\varrho}: \mathrm{e}^{\mathrm{H}}(S) \rightarrow \mathbb{R}^{n-1}$ to the space $\mathrm{e}^{\mathrm{H}}(S)$ of Hölder geodesic currents of $S$, a topological 
vector space that contains all conjugacy classes of $\pi_{1}(S)$ in a natural way; this continuous extension $\ell^{\varrho}: \mathrm{e}^{\mathrm{H}}(S) \rightarrow \mathbb{R}^{n-1}$ is unique on the subspaces of $\mathrm{C}^{\mathrm{H}}(S)$ that are of interest to us in this paper (see Remark 7.3).

A tangent cycle $\alpha \in \mathcal{Z}(\hat{\lambda} ; \mathbb{R})$, namely a relative tangent cycle $\alpha \in \mathcal{Z}(\hat{\lambda}$, slits; $\mathbb{R})$ with no correction factor, defines a Hölder geodesic current $\alpha \in \mathrm{e}^{\mathrm{H}}(S)$ (see [7]), and we can restrict the length function of $[15]$ to $\ell^{\varrho}: Z(\hat{\lambda} ; \mathbb{R}) \rightarrow \mathbb{R}^{n-1}$.

The following result, proved as Theorem 7.5 in $\S 7.2$, relates the length vector $\ell^{\varrho}(\alpha) \in$ $\mathbb{R}^{n-1}$ to the shearing class $\left[\sigma^{\varrho}\right] \in \mathcal{Z}\left(\hat{\lambda}\right.$, slits $\left.; \mathbb{R}^{n-1}\right) \cong H_{1}\left(\widehat{U}, \partial_{\mathrm{v}} \widehat{U} ; \mathbb{R}^{n-1}\right)$.

THEOREM 0.7. (Length and intersection formula) If $\left[\sigma^{\varrho}\right] \in \mathcal{Z}\left(\hat{\lambda}\right.$, slits; $\left.\mathbb{R}^{n-1}\right) \cong$ $H_{1}\left(\widehat{U}, \partial_{\mathrm{v}} \widehat{U} ; \mathbb{R}^{n-1}\right)$ is the shearing cycle of a Hitchin character $\varrho \in \operatorname{Hit}_{n}(S)$, and if $\alpha \in$ $z(\hat{\lambda} ; \mathbb{R}) \cong H_{1}(\widehat{U} ; \mathbb{R})$ is a tangent cycle for the orientation cover $\hat{\lambda}$, then the length vector

$$
\ell^{\varrho}(\alpha)=[\alpha] \cdot\left[\sigma^{\varrho}\right] \in \mathbb{R}^{n-1}
$$

is equal to the algebraic intersection vector of the homology classes $[\alpha] \in H_{1}(\widehat{U} ; \mathbb{R})$ and $\left[\sigma^{\varrho}\right] \in H_{1}\left(\widehat{U}, \partial_{\mathrm{v}} \widehat{U} ; \mathbb{R}^{n-1}\right)$ in the train-track neighborhood $\widehat{U}$ of $\hat{\lambda}$.

This is the natural extension of the formula which, in the classical case $n=2$, relates shearing coordinates and lengths of tangent cycles through Thurston's symplectic form $[5, \S 3]$.

In the special case where the tangent cycle $\alpha \in Z(\lambda ; \mathbb{R})$ is defined by a transverse measure $\mu$ for $\hat{\lambda}$, Theorem 0.7 establishes an equivalence between the positive intersection condition of Proposition 0.3 and the property that all coordinates of the vector $\ell^{\varrho}(\mu)$ are positive. In this version, this positivity property is an immediate consequence of the Anosov property that is central to [27] (see Proposition 7.4).

The article concludes, in $\S 9$ and $\S 10$, with two brief applications of Theorems 0.4 and 0.7. The first one is concerned with the dynamics of the action of a pseudo-Anosov diffeomorphism $\varphi: S \rightarrow S$ on the Hitchin component $\operatorname{Hit}_{n}(S)$; applying the parametrization of Theorem 0.4 to the case of a maximal geodesic lamination $\lambda$ containing the stable lamination of $\varphi$ shows that the dynamics of the action of $\varphi$ on $\operatorname{Hit}_{n}(S)$ are concentrated on submanifolds of $\operatorname{Hit}_{n}(S)$ of relatively large codimension. The second application considers the restriction of the length function $\ell^{\varrho}: \mathrm{e}^{\mathrm{H}}(S) \rightarrow \mathbb{R}^{n-1}$ to Thurston's space $\mathcal{M} \mathcal{L}(S)$ of measured laminations on $S$; a consequence of Theorem 0.7 is that, at each $\alpha \in \mathcal{M} \mathcal{L}(S)$, the tangent map $T_{\alpha} \ell^{\varrho}: T_{\alpha} \mathcal{M} \mathcal{L}(S) \rightarrow \mathbb{R}^{n-1}$ is linear on each of the (possibly infinitely many) linear faces that naturally decompose $T_{\alpha} \mathcal{M} \mathcal{L}(S)$ for the piecewise linear structure of $\mathcal{M} \mathcal{L}(S)$. In particular, $T_{\alpha} \ell^{\varrho}$ is linear at those generic $\alpha \in \mathcal{M} \mathcal{L}(S)$ where $T_{\alpha} \mathcal{M} \mathcal{L}(S)$ admits a vector space structure (with a single face).

It should be possible to place the results of this article in a broader perspective, by replacing the Lie group $\operatorname{PSL}_{n}(\mathbb{R})$ with another split real algebraic group $G$. Indeed, 
many properties of the Hitchin component remain valid in this more general context [23], [27], [18], [22]. In this extended framework, our triangle invariant $\tau^{\varrho}$ should then associate with each component of $S \backslash \lambda$ a positive triple in the flag space $B \backslash G$, where $B$ is a Borel subgroup of the Lie group and where $B \backslash G$ here denotes the left quotient. The shearing class would be a relative homology class $\left[\sigma^{\varrho}\right] \in H_{1}\left(\widehat{U}, \partial_{\mathrm{v}} \widehat{U} ; \mathfrak{h}\right)$ valued in the Cartan algebra $\mathfrak{h}$ of $G$, and equivariant with respect to the covering involution $\iota: \widehat{U} \rightarrow \widehat{U}$ and to the opposition involution of $\mathfrak{h}$ multiplied by -1 . The shearing cycle boundary condition would then state that the boundary $\partial\left[\sigma^{\varrho}\right] \in H_{0}\left(\partial_{\mathrm{v}} \widehat{U} ; \mathfrak{h}\right)$ is completely determined by the triangle invariant $\tau^{\varrho} \in(B \backslash G)^{4(g-1)}$, while the positive intersection condition would require that the algebraic intersection vector $[\mu] \cdot\left[\sigma^{\varrho}\right] \in \mathfrak{h}$ belong to the principal Weyl chamber of $\mathfrak{h}$. There are still a few missing technical steps in the existing literature to complete this program in its full generality (and an additional stumbling block in the limited expertise of the authors), but a large part of the extension to this more general context should mostly be entailed by the use of the right vocabulary.

Acknowledgement. The authors are pleased to acknowledge helpful conversations with Antonin Guilloux and Anne Parreau, at a time when they (the authors) were very confused. They are also very grateful to the three referees for extensive feedback and suggestions that have greatly improved the content and exposition of the article, and to Giuseppe Martone for further comments on the manuscript.

\section{Generic configurations of flags}

Flags in $\mathbb{R}^{n}$ play a fundamental role in our construction of invariants of Hitchin characters. This section is devoted to certain invariants of finite families of flags, borrowed from [18].

\subsection{Flags}

A flag in $\mathbb{R}^{n}$ is a family $F$ of nested linear subspaces $F^{(0)} \subset F^{(1)} \subset \ldots \subset F^{(n-1)} \subset F^{(n)}$ of $\mathbb{R}^{n}$ where each $F^{(a)}$ has dimension $a$.

A pair of flags $(E, F)$ is generic if every subspace $E^{(a)}$ of $E$ is transverse to every subspace $F^{(b)}$ of $F$. This is equivalent to the property that $\mathbb{R}^{n}=E^{(a)} \oplus F^{(n-a)}$ for every $a$.

Similarly, a triple of flags $(E, F, G)$ is generic if each triple of subspaces $E^{(a)}, F^{(b)}$ and $G^{(c)}$, respectively in $E, F$ and $G$, meets transversely in the sense that

$$
\operatorname{dim}\left(E^{(a)}+F^{(b)}+G^{(c)}\right)=\min \{a+b+c, n\} .
$$




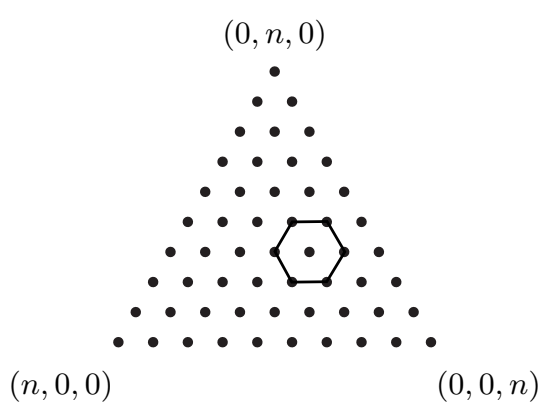

Figure 1. The discrete triangle $\Theta_{n}$, with a hexagon cycle.

Again, this is equivalent to the property that $\mathbb{R}^{n}=E^{(a)} \oplus F^{(b)} \oplus G^{(c)}$ for every $a, b$ and $c$ with $a+b+c=n$.

\subsection{Wedge-product invariants of generic flag triples}

Elementary linear algebra shows that, for any two generic flag pairs $(E, F)$ and $\left(E^{\prime}, F^{\prime}\right)$, there is a linear isomorphism $\mathbb{R}^{n} \rightarrow \mathbb{R}^{n}$ sending $E$ to $E^{\prime}$ and $F$ to $F^{\prime}$. However, the same is not true for generic flag triples. Indeed, there is a whole moduli space of generic flag triples modulo the action of $\mathrm{GL}_{n}(\mathbb{R})$, and this moduli space can be parametrized by invariants that we now describe. These invariants, originally introduced by Fock and Goncharov [18], are expressed in terms of the exterior algebra $\Lambda^{\bullet}\left(\mathbb{R}^{n}\right)$ of $\mathbb{R}^{n}$.

Consider the discrete triangle

$$
\Theta_{n}=\left\{(a, b, c) \in \mathbb{Z}^{3}: a+b+c=n \text { and } a, b, c \geqslant 0\right\}
$$

represented in Figure 1.

A function $\varphi: \Theta_{n} \rightarrow \mathbb{Z}$ is balanced if, for every $a_{0}, b_{0}$ and $c_{0}$,

$$
\sum_{\left(a_{0}, b, c\right) \in \Theta_{n}} \varphi\left(a_{0}, b, c\right)=\sum_{\left(a, b_{0}, c\right) \in \Theta_{n}} \varphi\left(a, b_{0}, c\right)=\sum_{\left(a, b, c_{0}\right) \in \Theta_{n}} \varphi\left(a, b, c_{0}\right)=0,
$$

namely if the sum of the $\varphi(a, b, c)$ over each line parallel to one side of the triangle $\Theta_{n}$ is equal to zero.

Such a balanced function $\varphi$ defines an invariant of a generic flag triple $(E, F, G)$ as follows. For each $a, b$ and $c$ between 0 and $n$, the spaces $\Lambda^{a}\left(E^{(a)}\right), \Lambda^{b}\left(F^{(b)}\right)$ and $\Lambda^{c}\left(G^{(c)}\right)$ are each isomorphic to $\mathbb{R}$. Choose non-zero elements $e^{(a)} \in \Lambda^{a}\left(E^{(a)}\right), f^{(b)} \in \Lambda^{b}\left(F^{(b)}\right)$ and $g^{(c)} \in \Lambda^{c}\left(G^{(c)}\right)$. We will use the same letters to denote their images $e^{(a)} \in \Lambda^{a}\left(\mathbb{R}^{n}\right), f^{(b)} \in$ $\Lambda^{b}\left(\mathbb{R}^{n}\right)$ and $g^{(c)} \in \Lambda^{c}\left(\mathbb{R}^{n}\right)$. We then define

$$
W_{\varphi}(E, F, G)=\prod_{(a, b, c) \in \Theta_{n}}\left(e^{(a)} \wedge f^{(b)} \wedge g^{(c)}\right)^{\varphi(a, b, c)} \in \mathbb{R}
$$


where we choose an isomorphism $\Lambda^{n}\left(\mathbb{R}^{n}\right) \cong \mathbb{R}$ to interpret each term in the product as a real number. The fact that the flag triple is generic guarantees that these numbers are non-zero, while the property that $\varphi$ is balanced is exactly what is needed to make sure that this product is independent of the choices of the elements $e^{(a)} \in \Lambda^{a}\left(E^{(a)}\right), f^{(b)} \in \Lambda^{b}\left(F^{(b)}\right)$ and $g^{(c)} \in \Lambda^{c}\left(G^{(c)}\right)$ and of the isomorphism $\Lambda^{n}\left(\mathbb{R}^{n}\right) \cong \mathbb{R}$. We say that $W_{\varphi}$ is the wedgeproduct invariant of generic flag triples associated with the balanced function $\varphi: \Theta \rightarrow \mathbb{Z}$.

We now consider a fundamental special case. For $a, b, c \geqslant 1$ with $a+b+c=n$, namely for a point $(a, b, c)$ in the interior of the triangle $\Theta_{n}$, the $(a, b, c)$-hexagon cycle is the balanced function $\varphi_{a b c}: \Theta_{n} \rightarrow \mathbb{Z}$ defined by

$$
\varphi_{a b c}=\delta_{(a+1, b, c-1)}-\delta_{(a-1, b, c+1)}+\delta_{(a, b-1, c+1)}-\delta_{(a, b+1, c-1)}+\delta_{(a-1, b+1, c)}-\delta_{(a+1, b-1, c)},
$$

where $\delta_{(a, b, c)}: \Theta_{n} \rightarrow \mathbb{Z}$ denotes the Kronecker function such that

$$
\delta_{(a, b, c)}\left(a^{\prime}, b^{\prime}, c^{\prime}\right)= \begin{cases}1, & \text { if }(a, b, c)=\left(a^{\prime}, b^{\prime}, c^{\prime}\right), \\ 0, & \text { otherwise. }\end{cases}
$$

The terminology is explained by the fact that the support of $\varphi_{a b c}$ is a small hexagon in the discrete triangle $\Theta_{n}$, centered at the point $(a, b, c)$; see Figure 1 for the case where $n=9$ and $(a, b, c)=(2,3,4)$. The wedge-product invariant associated with the hexagon cycle $\varphi_{a b c}$ is the $(a, b, c)$-triple ratio

$$
T_{a b c}(E, F, G)=\frac{e^{(a+1)} \wedge f^{(b)} \wedge g^{(c-1)}}{e^{(a-1)} \wedge f^{(b)} \wedge g^{(c+1)}} \frac{e^{(a)} \wedge f^{(b-1)} \wedge g^{(c+1)}}{e^{(a)} \wedge f^{(b+1)} \wedge g^{(c-1)}} \frac{e^{(a-1)} \wedge f^{(b+1)} \wedge g^{(c)}}{e^{(a+1)} \wedge f^{(b-1)} \wedge g^{(c)}} .
$$

Note that there is no triple ratio invariant when $n=2$, and exactly one triple ratio $T_{111}(E, F, G)$ when $n=3$. See $[18, \S 9.5]$ for a reduction of any triple ratio invariant $T_{a b c}(E, F, G)$ to the 3 -dimensional case, by consideration of the quotient space $\mathbb{R}^{n} /\left(E^{(a-1)}+F^{(b-1)}+G^{(c-1)}\right) \cong \mathbb{R}^{3}$, and its interpretation as a cross-ratio in the associated projective plane.

Triple ratios have the following properties under permutation of the flags.

LEMMA 1.1.

$$
T_{a b c}(E, F, G)=T_{b c a}(F, G, E)=T_{b a c}(F, E, G)^{-1} .
$$

Proof. The proof is elementary.

The natural action of the linear group $\mathrm{GL}_{n}(\mathbb{R})$ on the flag variety $\mathrm{Flag}\left(\mathbb{R}^{n}\right)$ descends to an action of the projective linear group $\mathrm{PGL}_{n}(\mathbb{R})$, quotient of $\mathrm{GL}_{n}(\mathbb{R})$ by its center $(\mathbb{R} \backslash\{0\}) \operatorname{Id}_{n}$ consisting of all non-zero scalar multiples of the identity matrix. Note that the projective special linear group $\mathrm{PSL}_{n}(\mathbb{R})$ is equal to $\mathrm{PGL}_{n}(\mathbb{R})$ if $n$ is odd, and is an index-2 subgroup of $\mathrm{PGL}_{n}(\mathbb{R})$ otherwise. 
Proposition 1.2. Two generic flag triples $(E, F, G)$ and $\left(E^{\prime}, F^{\prime}, G^{\prime}\right)$ are equivalent under the action of $\mathrm{PGL}_{n}(\mathbb{R})$ if and only if $T_{a b c}(E, F, G)=T_{a b c}\left(E^{\prime}, F^{\prime}, G^{\prime}\right)$ for every $a, b, c \geqslant 1$ with $a+b+c=n$.

In addition, for any set of non-zero numbers $t_{a b c} \in \mathbb{R} \backslash\{0\}$, there exists a generic flag triple $(E, F, G)$ such that $T_{a b c}(E, F, G)=t_{a b c}$ for every $a, b, c \geqslant 1$ with $a+b+c=n$.

Proof. See [18, §9].

In particular, the moduli space of generic flag triples $(E, F, G)$ under the action of $\mathrm{PGL}_{n}(\mathbb{R})$ is homeomorphic to $(\mathbb{R} \backslash\{0\})^{(n-1)(n-2) / 2}$.

Corollary 1.4 below partially accounts for the important role played by the triple ratios $T_{a b c}$ in Proposition 1.2. We will not really need this property, but it explains why we will always be able to express in terms of triple ratios $T_{a b c}$ the various wedge-product invariants that we will encounter in the paper.

LEMMA 1.3. The hexagon cycles $\left\{\varphi_{a b c}: a, b, c \geqslant 1\right.$ with $\left.a+b+c=n\right\}$ form a basis for the free abelian group consisting of all balanced functions $\varphi: \Theta_{n} \rightarrow \mathbb{Z}$.

Proof. The proof is elementary, by induction on $n$.

COROLlARY 1.4. Every wedge-product invariant can be uniquely expressed as a product of integer powers of triple ratios.

Proof. This is an immediate consequence of Lemma 1.3.

\subsection{Quadruple ratios}

In addition to triple ratios, the following wedge-product invariants of generic flag triples will play a very important role in this article.

For $a=1,2, \ldots, n-1$, the a-th quadruple ratio of the generic flag triple $(E, F, G)$ is the wedge-product invariant

$$
Q_{a}(E, F, G)=\frac{e^{(a-1)} \wedge f^{(n-a)} \wedge g^{(1)}}{e^{(a)} \wedge f^{(n-a-1)} \wedge g^{(1)}} \frac{e^{(a)} \wedge f^{(1)} \wedge g^{(n-a-1)}}{e^{(a-1)} \wedge f^{(1)} \wedge g^{(n-a)}} \frac{e^{(a+1)} \wedge f^{(n-a-1)}}{e^{(a+1)} \wedge g^{(n-a-1)}} \frac{e^{(a)} \wedge g^{(n-a)}}{e^{(a)} \wedge f^{(n-a)}},
$$

where, as usual, we consider arbitrary non-zero elements $e^{(b)} \in \Lambda^{b}\left(E^{(b)}\right), f^{(b)} \in \Lambda^{b}\left(F^{(b)}\right)$ and $g^{(b)} \in \Lambda^{b}\left(G^{(b)}\right)$, and where the ratios are computed in $\Lambda^{n}\left(\mathbb{R}^{n}\right) \cong \mathbb{R}$.

Note that $Q_{a}(E, G, F)=Q_{a}(E, F, G)^{-1}$, but that this quadruple ratio usually does not behave well under the other permutations of the flags $E, F$ and $G$, as $E$ plays a special role in $Q_{a}(E, F, G)$.

For this wedge-product invariant, we can explicitly determine the formula predicted by Corollary 1.4. 
LEMMA 1.5. For $a=1,2, \ldots, n-1$,

$$
Q_{a}(E, F, G)=\prod_{b+c=n-a} T_{a b c}(E, F, G)
$$

where the product is over all integers $b, c \geqslant 1$ with $b+c=n-a$. In particular,

$$
Q_{n-1}(E, F, G)=1 \quad \text { and } \quad Q_{n-2}(E, F, G)=T_{(n-2) 11}(E, F, G) .
$$

Proof. When computing the right-hand side of the equation, most of the terms $e^{\left(a^{\prime}\right)} \wedge f^{\left(b^{\prime}\right)} \wedge g^{\left(c^{\prime}\right)}$ cancel out and we are left with the eight terms of $Q_{a}(E, F, G)$.

\subsection{Double ratios}

We now consider quadruples $(E, F, G, H)$ of flags $E, F, G, H \in \mathrm{Flag}\left(\mathbb{R}^{n}\right)$. Such a flag quadruple is generic if each quadruple of subspaces $\left(E^{(a)}, F^{(b)}, G^{(c)}, H^{(d)}\right)$ meets transversely. As usual, we can restrict attention to the cases where $a+b+c+d=n$.

For $1 \leqslant a \leqslant n-1$, the $a$-th double ratio of the generic flag quadruple $(E, F, G, H)$ is

$$
D_{a}(E, F, G, H)=-\frac{e^{(a)} \wedge f^{(n-a-1)} \wedge g^{(1)}}{e^{(a)} \wedge f^{(n-a-1)} \wedge h^{(1)}} \frac{e^{(a-1)} \wedge f^{(n-a)} \wedge h^{(1)}}{e^{(a-1)} \wedge f^{(n-a)} \wedge g^{(1)}},
$$

where we choose arbitrary non-zero elements $e^{\left(a^{\prime}\right)} \in \Lambda^{a^{\prime}}\left(E^{\left(a^{\prime}\right)}\right), f^{\left(b^{\prime}\right)} \in \Lambda^{1}\left(F^{\left(b^{\prime}\right)}\right), g^{(1)} \in$ $\Lambda^{1}\left(G^{(1)}\right)$ and $h^{(1)} \in \Lambda^{1}\left(H^{(1)}\right)$. As usual, $D_{a}(E, F, G, H)$ is independent of these choices.

The following statement gives a better feeling of what is actually measured by this double ratio.

LEMMA 1.6. For a generic flag quadruple $(E, F, G, H)$, consider the decomposition $\mathbb{R}^{n}=\bigoplus_{a=1}^{n} L_{a}$, where $L_{a}=E^{(a)} \cap F^{(n-a+1)}$. For arbitrary non-zero vectors $g \in G^{(1)}$ and $h \in H^{(1)}$, let $g_{a}, h_{a} \in L_{a}$ be the respective projections of $g$ and $h$ to the line $L_{a}$ parallel to the other lines $L_{b}$ with $b \neq a$. Then

$$
D_{a}(E, F, G, H)=-\frac{g_{a+1}}{h_{a+1}} \frac{h_{a}}{g_{a}},
$$

where the ratios $g_{b} / h_{b} \in \mathbb{R}$ are measured in the lines $L_{b}$.

Proof. This results from a simple computation.

When $n=2,-D_{1}(E, F, G, H)$ is the familiar cross-ratio of the lines $E^{(1)}, F^{(1)}, G^{(1)}$, $H^{(1)}$ in $\mathbb{R}^{2}$. See $[18, \S 9.5]$ for a reduction of any double ratio invariant $D_{a}(E, F, G, H)$ to this 2-dimensional case, by consideration of the quotient space

$$
\frac{\mathbb{R}^{n}}{E^{(a-1)}+F^{(n-a-1)}} \cong \mathbb{R}^{2} .
$$


Note that $D_{a}(E, F, G, H)$ does not really depend on the whole flags $G$ and $H$, but only on the lines $G^{(1)}$ and $H^{(1)}$. The following elementary properties indicate how it behaves under transposition of $E$ and $F$, or of $G$ and $H$.

LEMMA 1.7.

$$
\begin{aligned}
& D_{a}(E, F, H, G)=D_{a}(E, F, G, H)^{-1} \\
& D_{a}(F, E, G, H)=D_{n-a}(E, F, G, H)^{-1}, \\
& D_{a}(E, F, G, K)=-D_{a}(E, F, G, H) D_{a}(E, F, H, K) .
\end{aligned}
$$

Proof. Again, the proof is elementary.

The minus sign in the definition of $D_{a}(E, F, G, H)$ is justified by the positivity property of the next section, and in particular by Proposition 1.8.

\subsection{Positivity}

An ordered family of flags $\left(E_{1}, E_{2}, \ldots, E_{m}\right) \in \mathrm{Flag}\left(\mathbb{R}^{n}\right)^{m}$ is positive if both the following conditions hold:

(1) for every distinct $i, j$ and $k$, and for every $a, b, c \geqslant 1$ with $a+b+c=n$, the triple ratio $T_{a b c}\left(E_{i}, E_{j}, E_{k}\right)$ is positive;

(2) for every distinct $i, j, k$ and $l$ with $i<k<j<l$ or $k<i<l<j$, and for every $1 \leqslant a \leqslant n-1$, the double ratio $D_{a}\left(E_{i}, E_{j}, E_{k}, E_{l}\right)$ is positive.

When $n=2$, the first condition is irrelevant. The second condition just means that, up to reflection, the flags $E_{1}, E_{2}, \ldots, E_{m} \in \mathrm{Flag}\left(\mathbb{R}^{2}\right)=\mathbb{R P}^{1}$ occur in this order around the projective line $\mathbb{R P}^{1}$.

Fock and Goncharov [18, §5] give a much more conceptual definition of positivity, building on earlier work of Lusztig [31], [32]. In particular, they prove the following result.

Proposition 1.8. ([18]) If the flag m-tuple $\left(E_{1}, E_{2}, \ldots, E_{m}\right)$ is positive, any flag $m$-tuple obtained by dihedral permutation of the $E_{i}$ is also positive.

Recall that a dihedral permutation is either a cyclic permutation, or the composition of the order reversal $\left(E_{1}, E_{2}, \ldots, E_{m}\right) \mapsto\left(E_{m}, E_{m-1}, \ldots, E_{1}\right)$ with a cyclic permutation.

\section{Geodesic laminations}

Geodesic laminations are a now very classical tool in 2-dimensional topology and geometry. They occur in many different contexts, for instance when one takes limits of 
sequences of simple closed curves. We state here a few basic definitions and facts, and refer to [39], [14], [36], [8] for proofs and background.

To define geodesic laminations, one first chooses a metric $m$ of negative curvature on the surface $S$.

An m-geodesic lamination is a closed subset $\lambda \subset S$ that can be decomposed as a disjoint union of simple complete $m$-geodesics, called its leaves. Recall that a geodesic is complete if it cannot be extended to a longer geodesic, and it is simple if it has no transverse self-intersection point. The leaves of a geodesic lamination can be closed or bi-infinite. A geodesic lamination can have finitely many leaves (as in the case considered in [9]), or uncountably many leaves.

An $m$-geodesic lamination has measure zero, and in fact Hausdorff dimension 1 [1], and its decomposition as a union of leaves is unique. The complement $S \backslash \lambda$ of an $m$ geodesic lamination $\lambda$ is a surface of finite topological type, bounded by finitely many leaves of $\lambda$. The completion of $S \backslash \lambda$ for the path metric induced by $m$ is a finite area surface with geodesic boundary; it is the union of a compact part and of finitely many spikes homeomorphic to $[0,1] \times[0, \infty[$, where $\{0,1\} \times[0, \infty[$ is contained in two leaves of $\lambda$. The width of these spikes decreases exponentially in the sense that the parametrization by $[0,1] \times[0, \infty[$ can be chosen so that its restriction to each $\{x\} \times[0, \infty[$ has speed 1 , and so that the length of each arc $[0,1] \times\{t\}$ decreases exponentially with $t$.

Because the leaves of $\lambda$ are disjoint, every point of $S$ has a neighborhood $U$ homeomorphic to $[0,1] \times[0,1]$ for which the intersection $U \cap \lambda$ corresponds to $K \times[0,1]$ for some totally disconnected compact subset $K \subset[0,1]$; beware that, in general, the homeomorphism cannot be made differentiable, only Hölder bicontinuous.

We will make heavy use of transverse arcs for $\lambda$. These are arcs differentiably immersed in $S$ that are transverse to the leaves of $\lambda$. In addition, we require that the endpoints of such a transverse arc be disjoint from $\lambda$.

The notion of geodesic lamination is independent of the choice of the negatively curved metric $m$ in the sense that, if $m^{\prime}$ is another negatively curved metric on $S$, there is a natural one-to-one correspondence between $m$-geodesic laminations and $m^{\prime}$-geodesic laminations.

A geodesic lamination $\lambda$ is maximal if it is not contained in any other geodesic lamination. This is equivalent to the property that each component of its complement $S \backslash \lambda$ is a triangle, bounded by three infinite leaves of $\lambda$ and containing three spikes of $S \backslash \lambda$. If the surface $S$ has genus $g$, an Euler characteristic argument shows that the number of triangle components of the complement $S \backslash \lambda$ of a maximal geodesic lamination is equal to $4(g-1)$.

Every geodesic lamination is contained in a maximal geodesic lamination. 
We can think of maximal geodesic laminations as some kind of triangulations of the surface $S$, where the edges are geodesic and where the vertices have been pushed to infinity. This point of view explains why maximal geodesic laminations are powerful tools for many problems, such as the ones considered in the current article.

\section{Triangle invariants}

Let $\varrho: \pi_{1}(S) \rightarrow \mathrm{PSL}_{n}(\mathbb{R})$ be a Hitchin homomorphism. We will use a maximal geodesic lamination $\lambda$ to construct invariants of the corresponding character $\varrho \in \operatorname{Hit}_{n}(S)$.

\subsection{The flag curve}

The key to the definition of these invariants is the following construction of Labourie [27].

Let $T^{1} S$ and $T^{1} \widetilde{S}$ be the unit tangent bundles of the surface $S$ and of its universal cover $\widetilde{S}$, respectively. For convenience, lift the homomorphism $\varrho: \pi_{1}(S) \rightarrow \mathrm{PSL}_{n}(\mathbb{R})$ to a homomorphism $\varrho^{\prime}: \pi_{1}(S) \rightarrow \mathrm{SL}_{n}(\mathbb{R})$. The fact that such a lift exists is classical when $n=2$, and therefore also holds when $\varrho: \pi_{1}(S) \rightarrow \mathrm{PSL}_{n}(\mathbb{R})$ comes from a discrete representation $\pi_{1}(S) \rightarrow \mathrm{PSL}_{2}(\mathbb{R})$; the existence of the lift in the general case follows by connectedness of the Hitchin component $\operatorname{Hit}_{n}(S)$, and by homotopy invariance of the obstruction to lift. We can then consider the twisted product

$$
T^{1} S \times \varrho_{\varrho^{\prime}} \mathbb{R}^{n}=\frac{T^{1} \widetilde{S} \times \mathbb{R}^{n}}{\pi_{1}(S)}
$$

where the fundamental group $\pi_{1}(S)$ acts on $T^{1} \widetilde{S}$ by its usual action on the universal cover $\widetilde{S}$, and acts on $\mathbb{R}^{n}$ by $\varrho^{\prime}$. The natural projection $T^{1} S \times{ }_{\varrho^{\prime}} \mathbb{R}^{n} \rightarrow T^{1} S$ presents $T^{1} S \times{ }_{\varrho^{\prime}} \mathbb{R}^{n}$ as a vector bundle over $T^{1} S$ with fiber $\mathbb{R}^{n}$.

Endow the surface $S$ with an arbitrary metric of negative curvature. This defines a circle at infinity $\partial_{\infty} \widetilde{S}$ for the universal cover $\widetilde{S}$, and a geodesic flow on the unit tangent bundle $T^{1} S$. It is well known (see for instance [20], [11] and [19]) that these objects are actually independent of the choice of the negatively curved metric, at least if we do not care about the actual parametrization of the geodesic flow (which is the case here).

The geodesic flow $\left(g_{t}\right)_{t \in \mathbb{R}}$ of $T^{1} S$ has a natural flat lift to a flow $\left(G_{t}\right)_{t \in \mathbb{R}}$ on the total space $T^{1} S \times_{\varrho^{\prime}} \mathbb{R}^{n}$. The flatness property here just means that the flow $\left(G_{t}\right)_{t \in \mathbb{R}}$ is the projection of the flow $\left(\widetilde{G}_{t}\right)_{t \in \mathbb{R}}$ on $T^{1} \widetilde{S} \times \mathbb{R}^{n}$ that acts by the geodesic flow $\left(\tilde{g}_{t}\right)_{t \in \mathbb{R}}$ of $T^{1} \widetilde{S}$ on the first factor, and by the identity $\operatorname{Id}_{\mathbb{R}^{n}}$ on the second factor.

Endow each fiber of the vector bundle $T^{1} S \times{ }_{\varrho^{\prime}} \mathbb{R}^{n} \rightarrow T^{1} S$ with a norm $\|\cdot\|$ depending continuously on the corresponding point of $T^{1} S$. 
Theorem 3.1. (Labourie [27, Theorem 4.1]) If $\varrho: \pi_{1}(S) \rightarrow \operatorname{PSL}_{n}(\mathbb{R})$ is a Hitchin homomorphism, the vector bundle $T^{1} S \times \varrho_{\varrho^{\prime}} \mathbb{R}^{n} \rightarrow T^{1} S$ admits a unique decomposition as a direct sum $L_{1} \oplus L_{2} \oplus \ldots \oplus L_{n}$ of $n$ line sub-bundles $L_{a} \rightarrow T^{1} S$ such that

(1) each line bundle $L_{a}$ is invariant under the lift $\left(G_{t}\right)_{t \in \mathbb{R}}$ of the geodesic flow;

(2) for every $a>b$, there exist constants $A_{a b}, B_{a b}>0$ such that, for every $v_{a} \in L_{a}$ and $v_{b} \in L_{b}$ in the same fiber of $T^{1} S \times \varrho^{\prime} \mathbb{R}^{n}$ and for every $t \geqslant 0$,

$$
\frac{\left\|G_{t}\left(v_{b}\right)\right\|}{\left\|v_{b}\right\|} \leqslant A_{a b} \frac{\left\|G_{t}\left(v_{a}\right)\right\|}{\left\|v_{a}\right\|} e^{-B_{a b} t} .
$$

The second property is clearly independent of the choice of the norm $\|\cdot\|$. It is referred to as the Anosov property of the Hitchin homomorphism $\varrho$. This relative property does not say anything about whether the flow $\left(G_{t}\right)_{t \in \mathbb{R}^{n}}$ expands or contracts the fibers of any individual sub-bundle $L_{a}$, but states that, when $a<b$, the flow $\left(G_{t}\right)_{t \in \mathbb{R}^{n}}$ contracts the fibers of $L_{b}$ much more than those of $L_{a}$. Writing this in a more intrinsic way, this means that $\left(G_{t}\right)_{t \in \mathbb{R}^{n}}$ induces on the line bundle $\operatorname{Hom}\left(L_{a}, L_{b}\right)$ a flow that is uniformly contracting when $a>b$.

Lift the sub-bundles $L_{a}$ of $T^{1} S \times \varrho_{\varrho^{\prime}} \mathbb{R}^{n}=\left(T^{1} \widetilde{S} \times \mathbb{R}^{n}\right) / \pi_{1}(S)$ to sub-bundles $\tilde{L}_{a}$ of $T^{1} \widetilde{S} \times \mathbb{R}^{n}$. Because the line sub-bundles $L_{a}$ are invariant under the lift $\left(G_{t}\right)_{t \in \mathbb{R}}$ of the geodesic flow, the fiber of $\tilde{L}_{a}$ over $\tilde{x} \in \widetilde{S}$ is of the form $\{\tilde{x}\} \times \tilde{L}_{a}(g)$ for some line $\tilde{L}_{a}(g) \subset \mathbb{R}^{n}$ depending only on the orbit $g$ of $\tilde{x}$ for the geodesic flow of $T^{1} \widetilde{S}$.

The line $\tilde{L}_{a}(g) \subset \mathbb{R}^{n}$ depends on the orbit $g$ of the geodesic flow of $T^{1} \widetilde{S}$ or, equivalently, on the corresponding oriented geodesic $g$ of $\widetilde{S}$. The Anosov property has the following relatively easy consequence. Define a flag $E(g) \in \mathrm{Flag}\left(\mathbb{R}^{n}\right)$ by the property that $E(g)^{(a)}=\tilde{L}_{1}(g) \oplus \tilde{L}_{2}(g) \oplus \ldots \oplus \tilde{L}_{a}(g)$; then $E(g)$ depends only on the positive endpoint of $g$. More precisely, we have the following result.

Proposition 3.2. (Labourie [27, Theorem 4.1]) For a Hitchin homomorphism

$$
\varrho: \pi_{1}(S) \longrightarrow \mathrm{PSL}_{n}(\mathbb{R})
$$

there exists a unique map $\mathcal{F}_{\varrho}: \partial_{\infty} \widetilde{S} \rightarrow \operatorname{Flag}\left(\mathbb{R}^{n}\right)$ such that

(1) $\mathcal{F}_{\varrho}$ is Hölder continuous for the visual metric of $\partial_{\infty} \widetilde{S}$ and for an arbitrary riemannian metric on $\operatorname{Flag}\left(\mathbb{R}^{n}\right)$;

(2) for every oriented geodesic $g$ of $\widetilde{S}$ with positive endpoint $\tilde{x}_{+} \in \partial_{\infty} \widetilde{S}$, the image $\mathcal{F}_{\varrho}\left(\tilde{x}_{+}\right)$is equal to the flag $E(g)$ defined above.

In addition, $\mathcal{F}_{\varrho}$ is $\varrho$-equivariant in the sense that $\mathcal{F}_{\varrho}(\gamma \tilde{x})=\varrho(\gamma)\left(\mathcal{F}_{\varrho}(\tilde{x})\right)$ for every $\tilde{x} \in \partial_{\infty} \widetilde{S}$ and $\gamma \in \pi_{1}(S)$. 
By definition, this map $\mathcal{F}_{\varrho}: \partial_{\infty} \widetilde{S} \rightarrow \operatorname{Flag}\left(\mathbb{R}^{n}\right)$ is the flag curve of the Hitchin homomorphism $\varrho: \pi_{1}(S) \rightarrow \mathrm{PSL}_{n}(\mathbb{R})$. It is independent of the choice of the lift $\varrho^{\prime}: \pi_{1}(S) \rightarrow$ $\mathrm{SL}_{n}(\mathbb{R})$ of $\varrho: \pi_{1}(S) \rightarrow \mathrm{PSL}_{n}(\mathbb{R})$, and of the negatively curved metric on $S$ used to define the geodesic flow of the unit tangent bundle $T^{1} S$.

Note that the line decomposition $\mathbb{R}^{n}=\bigoplus_{a=1}^{n} \tilde{L}_{a}(g)$, associated by Theorem 3.1 with an oriented geodesic $g$ of $\widetilde{S}$, is easily recovered from the flag curve $\mathcal{F}_{\varrho}: \partial_{\infty} \widetilde{S} \rightarrow \operatorname{Flag}\left(\mathbb{R}^{n}\right)$ by the property that $\tilde{L}_{a}(g)=\mathcal{F}_{\varrho}\left(\tilde{x}_{+}\right)^{(a)} \cap \mathcal{F}_{\varrho}\left(\tilde{x}_{-}\right)^{(n-a+1)}$ for the positive and negative endpoints $\tilde{x}_{+}, \tilde{x}_{-} \in \partial_{\infty} \widetilde{S}$ of $g$. The main content of Proposition 3.2 is that, for two oriented geodesics $g$ and $g^{\prime}$ of $\widetilde{S}$ that share the same positive endpoint $\tilde{x}_{+} \in \partial_{\infty} \widetilde{S}$, the corresponding line decompositions $\mathbb{R}^{n}=\bigoplus_{a=1}^{n} \tilde{L}_{a}(g)$ and $\mathbb{R}^{n}=\bigoplus_{a=1}^{n} \tilde{L}_{a}\left(g^{\prime}\right)$ may be different but define the same linear subspaces $\mathcal{F}_{\varrho}\left(\tilde{x}_{+}\right)^{(a)}=\bigoplus_{b=1}^{a} \tilde{L}_{b}(g)=\bigoplus_{b=1}^{a} \tilde{L}_{b}\left(g^{\prime}\right)$ for every $a=1,2, \ldots, n$.

See also [21] for a nice exposition of Theorem 3.1 and Proposition 3.2.

The flag curve $\mathcal{F}_{\varrho}$ has the following important positivity property.

TheOREm 3.3. (Fock-Goncharov [18, Theorem 1.15]) For every finite set of distinct points $x_{1}, x_{2}, \ldots, x_{k} \in \partial_{\infty} \widetilde{S}$ occurring in this order on the circle at infinity $\partial_{\infty} \widetilde{S}$, the flag $k$-tuple $\left(\mathcal{F}_{\varrho}\left(x_{1}\right), \mathcal{F}_{\varrho}\left(x_{2}\right), \ldots, \mathcal{F}_{\varrho}\left(x_{k}\right)\right)$ is positive in the sense of $\S 1.5$.

\subsection{Triangle invariants of Hitchin characters}

We now define a first set of invariants for the Hitchin character represented by a homomorphism $\varrho: \pi_{1}(S) \rightarrow \mathrm{PSL}_{n}(\mathbb{R})$.

The complement of the maximal geodesic lamination $\lambda$ consists of finitely many infinite triangles $T_{1}, T_{2}, \ldots, T_{m}$, each with three spikes.

Consider such a triangle component $T$ of $S \backslash \lambda$, and select one of its spikes $s$. Lift $T$ to an ideal triangle $\widetilde{T}$ in the universal cover $\widetilde{S}$, and let $\tilde{s}$ be the spike of $\widetilde{T}$ corresponding to $s$. The spike $\tilde{s}$ uniquely determines a point of the circle at infinity $\partial_{\infty} \widetilde{S}$, which we will also denote by $\tilde{s}$.

Label the spikes of $T$ as $s, s^{\prime}$ and $s^{\prime \prime}$ in counterclockwise order around $T$, and let $\tilde{s}, \tilde{s}^{\prime}, \tilde{s}^{\prime \prime} \in \partial_{\infty} \widetilde{S}$ be the corresponding points of the circle at infinity. The flag triple $\left(\mathcal{F}_{\varrho}(\tilde{s}), \mathcal{F}_{\varrho}\left(\tilde{s}^{\prime}\right), \mathcal{F}_{\varrho}\left(\tilde{s}^{\prime \prime}\right)\right)$, associated with $\tilde{s}, \tilde{s}^{\prime}, \tilde{s}^{\prime \prime} \in \partial_{\infty} \widetilde{S}$ by the flag curve

$$
\mathcal{F}_{\varrho}: \partial_{\infty} \widetilde{S} \longrightarrow \operatorname{Flag}\left(\mathbb{R}^{n}\right),
$$

is positive by Theorem 3.3. We can therefore consider the logarithms

$$
\tau_{a b c}^{\varrho}(s)=\log T_{a b c}\left(\mathcal{F}_{\varrho}(\tilde{s}), \mathcal{F}_{\varrho}\left(\tilde{s}^{\prime}\right), \mathcal{F}_{\varrho}\left(\tilde{s}^{\prime \prime}\right)\right)
$$

of its triple ratios, defined for every $a, b, c \geqslant 1$ with $a+b+c=n$. By $\varrho$-equivariance of the flag curve $\mathcal{F}_{\varrho}$, these triple ratio logarithms depend only on the triangle $T$ and on the spike $s$ of $T$, and not on the choice of the lift $\widetilde{T}$. 
The following statement indicates how the invariant $\tau_{a b c}^{\varrho}(s) \in \mathbb{R}$ changes if we choose a different vertex of the triangle $T$.

LEMMA 3.4. If $s, s^{\prime}$ and $s^{\prime \prime}$ are the three spikes of the component $T$ of $S \backslash \lambda$, indexed counterclockwise around $T$, then

$$
\tau_{a b c}^{\varrho}(s)=\tau_{b c a}^{\varrho}\left(s^{\prime}\right)=\tau_{c a b}^{\varrho}\left(s^{\prime \prime}\right)
$$

Proof. This is an immediate consequence of Lemma 1.1.

By invariance of triple ratios under the action of $\mathrm{PGL}_{n}(\mathbb{R})$ on $\mathrm{Flag}\left(\mathbb{R}^{n}\right)$, it is immediate that the triangle invariants $\tau_{a b c}^{\varrho}(s)$ depend only on the character $\varrho \in \operatorname{Hit}_{n}(S)$, and not on the homomorphism $\varrho: \pi_{1}(S) \rightarrow \mathrm{PSL}_{n}(\mathbb{R})$ representing it.

Because of Lemma 3.4, we can think of the invariant $\tau_{a b c}^{\varrho}(s)$ as mainly associated with the triangle component $T$ of $S \backslash \lambda$ that has the spike $s$ as a vertex, since choosing a different vertex of $T$ only affects the order in which the indices $a, b$ and $c$ are considered. For this reason, we will refer to the $\tau_{a b c}^{\varrho}(s)$ as the triangle invariants of the Hitchin character $\varrho \in \operatorname{Hit}_{n}(S)$.

Remark 3.5. The companion article [9] uses a clockwise labeling convention for the vertices of a triangle. As a consequence, the triangle invariants of [9] are the opposite of those introduced here.

\section{Tangent cycles for a geodesic lamination}

The second type of invariants associated with a Hitchin character $\varrho \in \operatorname{Hit}_{n}(S)$ are more closely tied to the maximal geodesic lamination $\lambda$, and have a homological flavor. This section is devoted to the definitions and basic properties of the corresponding objects.

Because of the scope of this article, we are restricting attention to maximal geodesic laminations. However, much of the discussion can be adapted to general geodesic laminations.

\subsection{Tangent cycles}

Let $\hat{\lambda}$ be the orientation cover of the geodesic lamination $\lambda$, consisting of all pairs $(x, o)$, where $x \in \lambda$ and $o$ is a continuous orientation of the leaves of $\lambda$ near $x$. The map $(x, o) \mapsto x$ defines a 2 -fold covering map $\hat{\lambda} \rightarrow \lambda$.

Intuitively, a tangent cycle for $\hat{\lambda}$ is a certain real-valued local weight for the leaves of $\hat{\lambda}$, and defines a 1-dimensional de Rham current supported in $\hat{\lambda}$ as in [38]. Tangent cycles were called "transverse cocycles" in [7] and in subsequent papers, with the discrepancy 
being explained by Poincaré duality. The change in terminology is motivated by the relative tangent cycles that will be introduced in $\S 4.4$.

Let $U$ be a neighborhood of the geodesic lamination $\lambda$ in $S$. If $U$ is small enough that it avoids at least one point of each component of $S \backslash \lambda$, the cover $\hat{\lambda} \rightarrow \lambda$ extends to a 2-fold cover $\widehat{U} \rightarrow U$ for some surface $\widehat{U}$.

A tangent cycle $\alpha$ for the geodesic lamination $\hat{\lambda}$ is the assignment of a number $\alpha(k) \in \mathbb{R}$ to each arc $k \subset \widehat{U}$ transverse to $\hat{\lambda}$ such that the the following conditions hold:

(1) $\alpha$ is finitely additive, in the sense that $\alpha(k)=\alpha\left(k_{1}\right)+\alpha\left(k_{2}\right)$ whenever the arc $k$ is split into two transverse $\operatorname{arcs} k_{1}$ and $k_{2}$;

(2) $\alpha$ is invariant under homotopy respecting $\hat{\lambda}$, in the sense that $\alpha(k)=\alpha\left(k^{\prime}\right)$ whenever the transverse arcs $k$ and $k^{\prime}$ are homotopic by a homotopy respecting $\hat{\lambda}$.

To be more precise in the definitions, a transverse arc is a closed differentiable arc embedded in the surface $\widehat{U}$, whose boundary is disjoint from $\hat{\lambda}$ and whose interior has transverse intersection with the leaves of $\hat{\lambda}$ that it meets. Such an arc is not assumed to be oriented. Two transverse $\operatorname{arcs} k$ and $k^{\prime}$ are homotopic respecting $\hat{\lambda}$ if there is a continuous homotopy $H:[0,1] \times[0,1] \rightarrow \widehat{U}$, restricting to homeomorphisms $[0,1] \times\{0\} \rightarrow k$ and $[0,1] \times\{1\} \rightarrow k^{\prime}$, such that $H^{-1}(\hat{\lambda})=K \times[0,1]$ for some closed subset $K \subset[0,1]$. (Experts will know that in general $H$ cannot be chosen differentiable, but this subtlety is irrelevant here.)

It easily follows from the above two conditions that $\alpha(k)=0$ for every arc $k$ disjoint from $\hat{\lambda}$. As a consequence, the notion of tangent cycle is independent of the choice of the neighborhood $U$.

A well-known example of tangent cycle are transverse measures for $\hat{\lambda}$. These can be defined as tangent cycles $\mu$ such that $\mu(k) \geqslant 0$ for every transverse $\operatorname{arc} k$. Indeed, this positivity property enhances the finite additivity condition (1) to countable additivity, so that a positive tangent cycle defines on each transverse arc $k$ a measure with support $k \cap \hat{\lambda}$ that is invariant under homotopy respecting $\hat{\lambda}$. See, for instance, [7, Proposition $18]$.

\subsection{Train-track neighborhoods}

To determine the space of tangent cycles for the geodesic lamination $\lambda$, we will use a very specific type of neighborhood $U$ for $\lambda$. Train tracks and train-track neighborhoods are standard tools to work with geodesic laminations. See, for instance, [39] and [36] for an introduction to these notions, with the warning that many slight variations in conventions and terminology occur in the existing literature.

A (trivalent) train-track neighborhood for the geodesic lamination $\lambda$ is a closed neigh- 
borhood $U$ of $\lambda$ which can be decomposed as a union of finitely many embedded rectangles $R_{i}$ such that the following conditions are satisfied:

(1) the boundary of each rectangle $R_{i} \cong[0,1] \times[0,1]$ is divided into a horizontal boundary $\partial_{\mathrm{h}} R_{i}=[0,1] \times\{0,1\}$ and a vertical boundary $\partial_{\mathrm{v}} R_{i}=\{0,1\} \times[0,1]$;

(2) each component of the intersection $R_{i} \cap R_{j}$ of two distinct rectangles $R_{i}$ and $R_{j}$ is, up to swapping $i$ and $j$, a component of $\partial_{\mathrm{v}} R_{i}$ contained in $\partial_{\mathrm{v}} R_{j}$ and containing one of the endpoints of $\partial_{\mathrm{v}} R_{j}$;

(3) each of the four endpoints of $\partial_{\mathrm{v}} R_{i}$ is contained in some rectangle $R_{j}$ different from $R_{i}$;

(4) the leaves of $\lambda$ are transverse to the $\operatorname{arcs}\{x\} \times[0,1]$ in each rectangle $R_{i} \cong$ $[0,1] \times[0,1]$;

(5) a fifth condition indicated below is satisfied.

By construction, the boundary $\partial U$ of the train-track neighborhood $U$ naturally splits into two pieces. The horizontal boundary $\partial_{\mathrm{h}} U$ is the union of the horizontal boundaries $\partial_{\mathrm{h}} R_{i}$ of all rectangles $R_{i}$. The vertical boundary consists of those points of $\partial U$ that are contained in the vertical boundary $\partial_{\mathrm{v}} R_{i}$ of some rectangle $R_{i}$.

We can now state the missing condition.

(5) no component of $S \backslash U$ is a disk with 0,1 or 2 components of the vertical boundary $\partial_{\mathrm{v}} U$ in its closure.

In particular, the $\operatorname{arcs}\{x\} \times[0,1]$ of each rectangle $R_{i} \cong[0,1] \times[0,1]$ provide a foliation of $U$, whose leaves are called the ties of the train-track neighborhood. A tie is generic if it meets the boundary of $U$ only at its endpoints. Otherwise, it is singular.

The origin of the train track terminology should become apparent when $U$ is chosen so that its ties are relatively short. See Figure 2. In particular, a singular tie is also often called a switch, and the rectangles $R_{i}$ are the branches of $U$. The branches of the train-track neighborhood $U$ correspond to the edges of the (1-dimensional) train track associated with $U$, which explains our frequent use of the letter $e$ to denote a branch.

The definitions are such that a singular tie $t$ is adjacent to three branches $R_{i}, R_{j}$ and $R_{k}$, in such a way that $t$ is equal to a component of the vertical boundary $\partial_{\mathrm{v}} R_{i}$, and is also the union of a component of $\partial_{\mathrm{v}} R_{j}$, of a component of $\partial_{\mathrm{v}} R_{k}$ and of a component of $\partial_{\mathrm{v}} U$. The rectangles $R_{j}$ and $R_{k}$ are not necessarily distinct.

Every geodesic lamination admits a train-track neighborhood; see for instance [39] and [36].

The above definitions make sense for any geodesic lamination. When the geodesic lamination $\lambda$ is maximal, there is a crucial property of its train-track neighborhoods $U$ that we will use on a regular basis. 


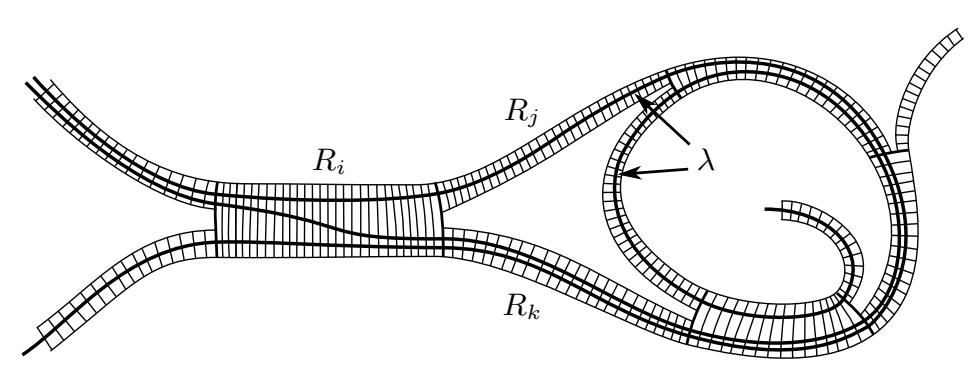

Figure 2. A train-track neighborhood.

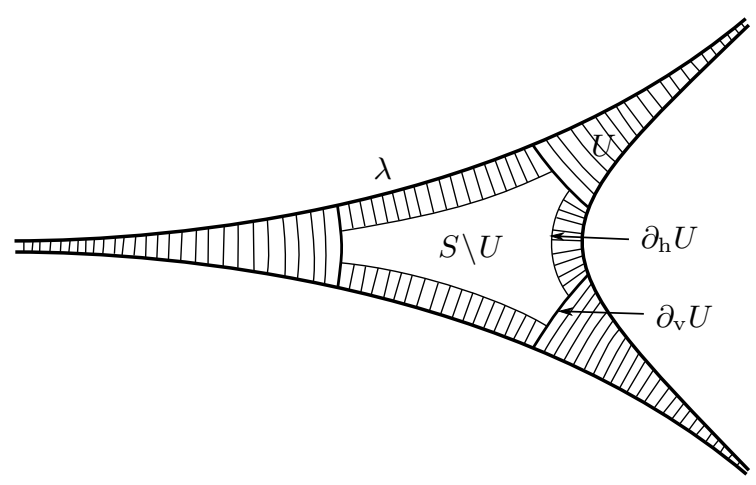

Figure 3. Train-track neighborhoods and maximal geodesic laminations.

Proposition 4.1. Let $U$ be a train-track neighborhood of the maximal geodesic lamination $\lambda$. Then, every component $T$ of the complement $S \backslash \lambda$ contains exactly one component $H=T \backslash U$ of $S \backslash U$; this component $H$ is a hexagon, namely a disk whose boundary is the union of three components of the horizontal boundary $\partial_{\mathrm{h}} U$ and three components of the vertical boundary $\partial_{\mathrm{v}} U$. In addition, the foliation of $T \cap U$ by the ties of $U$ is as indicated in Figure 3.

Proof. Since $\lambda$ is assumed to be maximal, its complement consists of infinite triangles. The property is then easily proved by extending the foliation of $U$ by its ties to a foliation of $S$ with saddle-type singularities, and by using an index computation on each component of the complement $S \backslash \lambda$.

Incidentally, another index argument applied to the whole surface $S$ shows that the complement $S \backslash U$ consists of $4(g-1)$ hexagons. In particular, this proves that the complement $S \backslash \lambda$ consists of $4(g-1)$ triangles. It also follows that the train-track neighborhood $U$ has $12(g-1)$ switches and $18(g-1)$ branches. 


\subsection{Homological interpretation of tangent cycles}

Train-track neighborhoods provide a convenient tool to perform computations in the vector space $\mathcal{C}(\hat{\lambda} ; \mathbb{R})$ consisting of all tangent cycles for the orientation cover $\hat{\lambda}$ of $\lambda$.

Let $U$ be a train-track neighborhood of the maximal geodesic lamination $\lambda$. Using Proposition 4.1, the orientation cover map $\hat{\lambda} \rightarrow \lambda$ has a unique extension to a cover $\widehat{U} \rightarrow U$. Note that $\hat{\lambda}$ is a geodesic lamination in the surface $\widehat{U}$, and that $\widehat{U}$ is a train-track neighborhood of $\hat{\lambda}$. Also, each component of $\widehat{U} \backslash \hat{\lambda}$ is an annulus bounded on one side by a chain of six leaves of $\hat{\lambda}$, and on the other side by a dodecagon made up of six components of the horizontal boundary $\partial_{\mathrm{h}} \widehat{U}$ and six components of the vertical boundary $\partial_{\mathrm{v}} \widehat{U}$.

The leaves of the orientation cover $\hat{\lambda}$ are canonically oriented (use the orientation $o$ near the point $(x, o) \in \hat{\lambda})$. This enables us to orient each tie $k$ of $\widehat{U}$ from left to right with respect to this canonical orientation of the leaves of $\hat{\lambda}$. Indeed, Proposition 4.1 guarantees that, for each component $d$ of $k \backslash \hat{\lambda}$, the left-to-right orientations of $k$ at the endpoints of $d$ coincide; as a consequence, all leaves of $\hat{\lambda}$ meeting $k$ induce the same left-to-right orientation for $k$.

Proposition 4.2. A tangent cycle $\alpha \in \mathcal{Z}(\hat{\lambda} ; \mathbb{R})$ uniquely determines a homology class $[\alpha] \in H_{1}(\widehat{U} ; \mathbb{R})$ by the property that

$$
\alpha(k)=[k] \cdot[\alpha]
$$

for every generic tie $k$ of the train-track neighborhood $\widehat{U}$, where $[k] \cdot[\alpha]$ is the algebraic intersection number of $[\alpha] \in H_{1}(\widehat{U} ; \mathbb{R})$ with the relative homology class $[k] \in H_{1}(\widehat{U}, \partial \widehat{U} ; \mathbb{R})$ defined by the tie $k$, endowed with the above left-to-right orientation.

In addition, the rule $\alpha \mapsto[\alpha]$ defines a linear isomorphism $z(\hat{\lambda} ; \mathbb{R}) \rightarrow H_{1}(\widehat{U} ; \mathbb{R})$.

Proof. Because the geodesic lamination $\lambda$ is maximal, Proposition 4.1 shows that it is tightly carried by the train track $U$, in the sense that each component of $U \backslash \lambda$ is an annulus. It follows that $\hat{\lambda}$ is tightly carried by $\widehat{U}$. The result is then a consequence of $[7$, Theorem 11].

Lemma 4.3. If the surface $S$ has genus $g$,

$$
\mathcal{C}(\hat{\lambda} ; \mathbb{R}) \cong H_{1}(\widehat{U} ; \mathbb{R}) \cong \mathbb{R}^{12 g-11} .
$$

Proof. Since the complement $S \backslash \lambda$ consists of infinite triangles, the geodesic lamination $\lambda$ is non-orientable. This implies that $\hat{\lambda}$ is connected, and therefore so is $\widehat{U}$. By definition of the Euler characteristic $\chi$,

$$
\operatorname{dim} H_{1}(\widehat{U} ; \mathbb{R})=-\chi(\widehat{U})+\operatorname{dim} H_{0}(\widehat{U} ; \mathbb{R})=-\chi(\widehat{U})+1=-2 \chi(U)+1 .
$$

We observed that the complement of $U$ in $S$ consists of $4(g-1)$ hexagons. Therefore, $\chi(U)=\chi(S)-4(g-1)=-6(g-1)$. The result follows. 


\subsection{Tangent cycles relative to the slits}

We now relax the additivity condition for a tangent cycle.

Let $U$ be a neighborhood of $\lambda$ in $S$ that avoids at least one point of each component of $S \backslash \lambda$. For instance, $U$ can be a train-track neighborhood of $\lambda$. Extend the orientation cover $\hat{\lambda} \rightarrow \lambda$ to a 2-fold cover $\widehat{U} \rightarrow U$. The complement $\widehat{U} \backslash \hat{\lambda}$ has a certain number of infinite spikes, in fact $24(g-1)$ spikes because the complement $S \backslash \lambda$ consists of $4(g-1)$ infinite triangles and because each spike of $S \backslash \lambda$ lifts to two spikes of $\widehat{U} \backslash \hat{\lambda}$. In particular, the spikes of $\widehat{U} \backslash \hat{\lambda}$ are really independent of the choice of the neighborhood $U$. For this reason, we will also refer to the spikes of $\widehat{U} \backslash \hat{\lambda}$ as the slits of $\hat{\lambda}$.

We need to restrict our attention to a special class of transverse arcs for $\hat{\lambda}$. An arc $k \subset \widehat{U}$ is tightly transverse to the geodesic lamination $\hat{\lambda}$ if it is transverse to $\hat{\lambda}$, if it has non-empty intersection with $\hat{\lambda}$ and if, for every component $d$ of $k \backslash \hat{\lambda}$, one of the following holds:

- $d$ contains one of the endpoints of $k$;

- $d$ separates one of the spikes of $\widehat{U} \backslash \hat{\lambda}$ from the rest of $\widehat{U} \backslash \hat{\lambda}$.

A fundamental example arises when $U$ is a train-track neighborhood of the maximal geodesic lamination $\lambda$, so that its lift $\widehat{U}$ is a train-track neighborhood of $\hat{\lambda}$. It then follows from Proposition 4.1 that every tie of $\widehat{U}$ is tightly transverse to $\hat{\lambda}$.

The slits of $\hat{\lambda}$, namely the spikes of $\widehat{U} \backslash \hat{\lambda}$, come in two types because of the canonical orientation of the leaves of the orientation cover $\hat{\lambda}$ : the positive slits $s$ where the two leaves of $\hat{\lambda}$ that are adjacent to $s$ are oriented towards $s$ for the canonical orientation of $\hat{\lambda}$, and the negative slits where these two leaves are oriented away from $s$. Define the sign $\varepsilon$ of the slit $s$ of $\widehat{U} \backslash \hat{\lambda}$ by

$$
\varepsilon(s)=\left\{\begin{array}{cl}
+1, & \text { if } s \text { is positive } \\
-1, & \text { if } s \text { is negative }
\end{array}\right.
$$

For mnemonic purposes, it may be useful to observe that our positive/negative terminology is compatible with the boundary orientation coming from orientation of the leaves of $\hat{\lambda}$. A positive slit corresponds to the positive endpoints of the two leaves of $\hat{\lambda}$ that are adjacent to it, whereas a negative slit corresponds to their negative endpoints.

An $\mathbb{R}$-valued tangent cycle relative to the slits for $\hat{\lambda}$ assigns a number $\alpha(k) \in \mathbb{R}$ to each arc $k \subset \widehat{U}$ tightly transverse to $\hat{\lambda}$ in such a way that

- $\alpha$ is, as before, invariant under homotopy respecting $\hat{\lambda}$ in the sense that $\alpha(k)=$ $\alpha\left(k^{\prime}\right)$ whenever the transverse arcs $k$ and $k^{\prime}$ are homotopic by a homotopy that keeps each point of $k \cap \hat{\lambda}$ in the same leaf of $\hat{\lambda}$;

- $\alpha$ is quasi-additive in the following sense: there is a number $\partial \alpha(s) \in \mathbb{R}$ associated 
with each slit $s$ of $\hat{\lambda}$ such that

$$
\alpha(k)=\alpha\left(k_{1}\right)+\alpha\left(k_{2}\right)-\varepsilon(s) \partial \alpha(s)
$$

whenever the arc $k \subset \widehat{U}$ is tightly transverse to $\hat{\lambda}$, the arcs $k_{1}$ and $k_{2}$ are obtained by splitting $k$ at a point $x \in k \backslash \hat{\lambda}$ contained in a component $d$ of $k \backslash \hat{\lambda}$ that is disjoint from $\partial k$, $s$ is the spike separated from the rest of $\widehat{U} \backslash \hat{\lambda}$ by the component $d$, and $\varepsilon(s)= \pm 1$ is its sign as defined above.

The function $\partial \alpha:\{$ slits of $\hat{\lambda}\} \rightarrow \mathbb{R}$ is the boundary of the relative cycle $\alpha$. We could have combined $\partial \alpha$ with the $\operatorname{sign} \varepsilon$ to create a single function $\{$ slits of $\hat{\lambda}\} \rightarrow \mathbb{R}$, but the current convention simplifies the homological interpretation of relative tangent cycles that is given in Proposition 4.5 below. This homological interpretation will also explain the boundary terminology.

We let $z(\hat{\lambda}$, slits; $\mathbb{R})$ denote the space of tangent cycles relative to the slits for $\hat{\lambda}$.

Using the quasi-additivity property, one easily shows that the notion of tangent cycle relative to the slits is independent of the choice of the neighborhood $U$ of $\lambda$.

These relative tangent cycles generalize the tangent cycles of $\S 4.1$, as indicated by the following statement.

LEMMA 4.4. There is a natural correspondence between the set $\mathcal{Z}(\hat{\lambda} ; \mathbb{R})$ of tangent cycles for $\hat{\lambda}$ and the set $\{\alpha \in \mathcal{Z}(\hat{\lambda}$, slits; $\mathbb{R}): \partial \alpha=0\}$ of tangent cycles relative to the slits with boundary zero.

Proof. A relative tangent cycle with boundary equal to zero is additive. So the only point that requires some discussion is the fact that relative tangent cycles are restricted to arcs tightly transverse to $\hat{\lambda}$, whereas the definition of tangent cycles involves all arcs transverse to $\hat{\lambda}$. In particular, a transverse arc $k$ is allowed to backtrack so that a component $d$ of $k \backslash \hat{\lambda}$ has both endpoints on the same leaf of $\hat{\lambda}$, or to cut a component of $\widehat{U} \backslash \hat{\lambda}$ diagonally so that the endpoints of a component $d$ of $k \backslash \hat{\lambda}$ sit on leaves of $\hat{\lambda}$ that are not asymptotic to each other; both possibilities are forbidden for tightly transverse arcs.

However, every arc $k$ transverse to $\hat{\lambda}$ can be split into the union of finitely many arcs $k_{1}, k_{2}, \ldots, k_{l}$ that are tightly transverse to $\hat{\lambda}$. It easily follows that every relative tangent cycle $\alpha \in \mathcal{Z}(\hat{\lambda}$, slits; $\mathbb{R})$ with $\partial \alpha=0$ uniquely extends to a tangent cycle, by the property that $\alpha(k)=\sum_{i=1}^{l} \alpha\left(k_{i}\right)$ for every transverse arc $k$ split as above into finitely many tightly transverse $\operatorname{arcs} k_{i}$. Indeed, the additivity property guarantees that this $\alpha(k)$ does not depend on the decomposition of $k$ into tightly transverse arcs. 


\subsection{Homological interpretation of tangent cycles relative to the slits}

We now focus on a train-track neighborhood $U$ of the maximal geodesic laminations $\lambda$. As before, let $\hat{\lambda}$ be the orientation cover of $\lambda$, and extend the covering map $\hat{\lambda} \rightarrow \lambda$ to a cover $\widehat{U} \rightarrow U$. The canonical orientation of the leaves of $\hat{\lambda}$ provides a left-to-right orientation for the ties of $\widehat{U}$.

By Proposition 4.1, there is a one-to-one correspondence between the slits of $\hat{\lambda}$ and the components of the vertical boundary $\partial_{\mathrm{v}} \widehat{U}$. Indeed, each component $c$ of $\partial_{\mathrm{v}} \widehat{U}$ faces a unique spike $s$ of $U \backslash \hat{\lambda}$ (that is, a slit of $\hat{\lambda}$ ) in the sense that, if $k$ is the singular tie of $\widehat{U}$ that contains $c$ and if $d$ is the component of $k \backslash \hat{\lambda}$ that contains $c$, then $d$ separates $s$ from the rest of $\widehat{U} \backslash \hat{\lambda}$; see Figure 3 .

For a relative tangent cycle $\alpha \in \mathcal{Z}(\hat{\lambda}$, slits; $\mathbb{R})$, the boundary $\partial \alpha:\{$ slits of $\hat{\lambda}\} \rightarrow \mathbb{R}$ therefore assigns a real-valued weight to each component of $\partial_{\mathrm{v}} \widehat{U}$, and therefore can be interpreted as an element of $H_{0}\left(\partial_{\mathrm{v}} \widehat{U} ; \mathbb{R}\right)$.

Proposition 4.5. Let $U$ be a train-track neighborhood of the maximal geodesic lamination $\lambda$, and let $\widehat{U}$ be its lift to a train-track neighborhood of the orientation cover $\hat{\lambda}$. A tangent cycle $\alpha \in \mathcal{Z}(\hat{\lambda}$, slits; $\mathbb{R})$ relative to the slits of $\hat{\lambda}$ uniquely determines a relative homology class $[\alpha] \in H_{1}\left(\widehat{U}, \partial_{\mathrm{v}} \widehat{U} ; \mathbb{R}\right)$ by the property that

$$
\alpha(k)=[k] \cdot[\alpha]
$$

for every generic tie $k$ of the train-track neighborhood $\widehat{U}$, where $[k] \cdot[\alpha]$ is the algebraic intersection number of $[\alpha] \in H_{1}\left(\widehat{U}, \partial_{\mathrm{v}} \widehat{U} ; \mathbb{R}\right)$ with the relative homology class

$$
[k] \in H_{1}\left(\widehat{U}, \partial_{\mathrm{h}} \widehat{U} ; \mathbb{R}\right)
$$

defined by the tie $k$, endowed with the above left-to-right orientation.

In addition, the rule $\alpha \mapsto[\alpha]$ defines a linear isomorphism

$$
z(\hat{\lambda}, \operatorname{slits} ; \mathbb{R}) \cong H_{1}\left(\widehat{U}, \partial_{\mathrm{v}} \widehat{U} ; \mathbb{R}\right)
$$

for which the boundary $\partial \alpha:\{$ slits of $\hat{\lambda}\} \rightarrow \mathbb{R}$ of the relative tangent cycle $\alpha$ corresponds to the image of $[\alpha] \in H_{1}\left(\widehat{U}, \partial_{\mathrm{v}} \widehat{U} ; \mathbb{R}\right)$ under the boundary homomorphism

$$
\partial: H_{1}\left(\widehat{U}, \partial_{\mathrm{v}} \widehat{U} ; \mathbb{R}\right) \longrightarrow H_{0}\left(\partial_{\mathrm{v}} \widehat{U} ; \mathbb{R}\right) .
$$

Proof. We split the proof into a few steps to improve readability.

Step 1. Construct a linear map $\varphi: \mathcal{Z}(\hat{\lambda}$, slits; $\mathbb{R}) \rightarrow H_{1}\left(\widehat{U}, \partial_{\mathrm{v}} \widehat{U} ; \mathbb{R}\right)$. 
Pick a generic tie $k_{e}$ in each branch $e$ of $\widehat{U}$. An easy homological computation (for instance using a Mayer-Vietoris-type argument) shows that, as $e$ ranges over all branches of $\widehat{U}$, the relative homology classes $\left[k_{e}\right]$ form a basis for $H_{1}\left(\widehat{U}, \partial_{\mathrm{h}} \widehat{U} ; \mathbb{R}\right)$.

If we are given a relative tangent cycle $\alpha \in \mathcal{Z}(\hat{\lambda}$, slits; $\mathbb{R})$, the map $\left[k_{e}\right] \mapsto \alpha\left(k_{e}\right)$ therefore extends to a linear map $H_{1}\left(\widehat{U}, \partial_{\mathrm{h}} \widehat{U} ; \mathbb{R}\right) \rightarrow \mathbb{R}$. By Poincaré-Lefschetz duality and since the boundary $\partial \widehat{U}$ is the union of $\partial_{\mathrm{h}} \widehat{U}$ and $\partial_{\mathrm{v}} \widehat{U}$, there consequently exists a unique class $[\alpha] \in H_{1}\left(\widehat{U}, \partial_{\mathrm{v}} \widehat{U} ; \mathbb{R}\right)$ such that $\alpha\left(k_{e}\right)=\left[k_{e}\right] \cdot[\alpha]$ for every branch $e$.

An arbitrary generic tie $k$ of $\widehat{U}$ is contained in a branch $e$. Therefore, $[k]=\left[k_{e}\right]$ in $H_{1}\left(\widehat{U}, \partial_{\mathrm{h}} \widehat{U} ; \mathbb{R}\right)$, and $\alpha(k)=\alpha\left(k_{e}\right)$ by invariance of $\alpha$ under homotopy respecting $\hat{\lambda}$. This proves that $\alpha(k)=[k] \cdot[\alpha]$ for every generic tie $k$ of $\widehat{U}$. As a consequence, $[\alpha]$ satisfies the identity indicated in the statement of Proposition 4.5.

This provides a map $\varphi: z(\hat{\lambda}$, slits; $\mathbb{R}) \rightarrow H_{1}\left(\widehat{U}, \partial_{\mathrm{v}} \widehat{U} ; \mathbb{R}\right)$, associating the above class $[\alpha] \in H_{1}\left(\widehat{U}, \partial_{\mathrm{v}} \widehat{U} ; \mathbb{R}\right)$ with $\alpha \in \mathcal{Z}(\hat{\lambda}$, slits; $\mathbb{R})$.

Step 2. Construct a linear map $\psi: H_{1}\left(\widehat{U}, \partial_{\mathrm{v}} \widehat{U} ; \mathbb{R}\right) \rightarrow Z(\hat{\lambda}$, slits; $\mathbb{R})$.

We first associate a homology class $[k] \in H_{1}\left(\widehat{U}, \partial_{\mathrm{h}} \widehat{U} ; \mathbb{R}\right)$ with each arc $k$ that is tightly transverse to $\hat{\lambda}$.

A key observation is that the canonical orientation of $\hat{\lambda}$ specifies a natural orientation for $k$. Indeed, the definition of tight transversality implies that, if the $\operatorname{arc} k$ is tightly transverse to $\hat{\lambda}$, the canonical orientations of the leaves of $\hat{\lambda}$ passing through the endpoints of a component $d$ of $k \backslash \hat{\lambda}$ induce the same left-to-right orientation for $k$. As a consequence, all leaves of $\hat{\lambda}$ define the same left-to-right orientation for $k$. We orient $k$ with this orientation.

We now extend the tightly transverse arc $k$ to an $\operatorname{arc} k^{\prime} \subset \widehat{U}$ with $\partial k^{\prime} \subset \partial_{\mathrm{h}} \widehat{U}$. There is a natural one-to-one correspondence between the components of the horizontal boundary $\partial_{\mathrm{h}} \widehat{U}$ and the boundary leaves of $\hat{\lambda}$ (namely those which are in the boundary of $\widehat{U} \backslash \hat{\lambda}$, counting isolated leaves twice); indeed, Proposition 4.1 shows that all ties originating from a component of $\partial_{\mathrm{h}} \widehat{U}$ leave $\widehat{U} \backslash \hat{\lambda}$ on the same boundary leaf of $\hat{\lambda}$. For each component $d$ of $k \backslash \hat{\lambda}$ containing an endpoint of $k$, we can extend $d$ to an arc $d^{\prime} \subset \widehat{U} \backslash \hat{\lambda}$ going from a boundary leaf $l$ of $\hat{\lambda}$ to the component of $\partial_{\mathrm{h}} \widehat{U}$ that faces $l$ in the component of $\widehat{U} \backslash \hat{\lambda}$ containing $d$, in the homotopy class specified by the arcs in ties of $\widehat{U}$ that connect $l$ to $\partial_{\mathrm{h}} \widehat{U}$. Performing this operation for each of the two components $d$ of $k \backslash \hat{\lambda}$ that contain an endpoint of $k$, we have extended $k$ to an oriented arc $k^{\prime} \supset k$ whose boundary is contained in $\partial_{\mathrm{h}} \widehat{U}$. There are many possible choices for $k^{\prime}$ but all give the same relative homology class in $H_{1}\left(\widehat{U}, \partial_{\mathrm{h}} \widehat{U} ; \mathbb{R}\right)$, which we denote by $[k]$.

Given a relative homology class $c \in H_{1}\left(\widehat{U}, \partial_{\mathrm{v}} \widehat{U} ; \mathbb{R}\right)$, we can consider, for every arc $k$ tightly transverse to $\hat{\lambda}$, the algebraic intersection number

$$
\alpha_{c}(k)=[k] \cdot c \in \mathbb{R}
$$




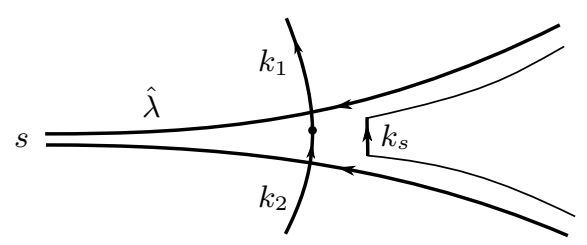

The positive slit case.

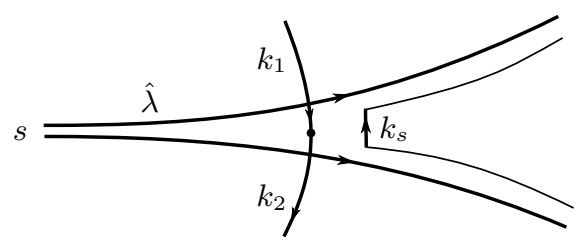

The negative slit case.

Figure 4. Splitting a tightly transverse arc.

of $c$ with the homology class $[k] \in H_{1}\left(\widehat{U}, \partial_{\mathrm{h}} \widehat{U} ; \mathbb{R}\right)$ associated with $k$ as above. We want to show that this defines a relative tangent cycle $\mathcal{Z}(\hat{\lambda}$, slits; $\mathbb{R})$.

The invariance of $\alpha_{c}(k)$ under homotopy of $k$ respecting $\hat{\lambda}$ is immediate.

We need to check the quasi-additivity property. Let the arc $k \subset \widehat{U}$ be tightly transverse to $\hat{\lambda}$, let $k_{1}$ and $k_{2}$ be obtained by splitting $k$ at a point $x \in k \backslash \hat{\lambda}$ contained in a component $d$ of $k \backslash \hat{\lambda}$ that is disjoint from $\partial k$, and let $s$ be the spike separated from the rest of $\widehat{U} \backslash \hat{\lambda}$ by the component $d$. Let $k_{s}$ be the component of $\partial_{\mathrm{v}} \widehat{U}$ that faces the slit $s$. Orient $k_{s}$ by the boundary orientation of $\partial \widehat{U}$.

Then, by the definition of the relative homology classes $[k],\left[k_{1}\right],\left[k_{2}\right] \in H_{1}\left(\widehat{U}, \partial_{\mathrm{h}} \widehat{U} ; \mathbb{R}\right)$, including the left-to-right choice of orientation for $k$, one has

$$
[k]=\left[k_{1}\right]+\left[k_{2}\right]+\varepsilon(s)\left[k_{s}\right] \in H_{1}\left(\widehat{U}, \partial_{\mathrm{h}} \widehat{U} ; \mathbb{R}\right),
$$

where $\varepsilon(s)= \pm 1$ is the sign of the slit $s$. See Figure 4. Taking intersection numbers with $c \in H_{1}\left(\widehat{U}, \partial_{\mathrm{v}} \widehat{U} ; \mathbb{R}\right)$, it follows that

$$
\alpha_{c}(k)=\alpha_{c}\left(k_{1}\right)+\alpha_{c}\left(k_{2}\right)+\varepsilon(s)\left[k_{s}\right] \cdot c .
$$

This proves that $\alpha_{c}$ is a tangent cycle for $\hat{\lambda}$ relative to its slits, with boundary $\partial \alpha_{c}$ defined by the property that $\partial \alpha_{c}(s)=-\left[k_{s}\right] \cdot c$ for every slit $s$. The minus sign comes from our convention in the definition of quasi-additivity for relative tangent cycles in $\S 4.4$.

We define $\psi: H_{1}\left(\widehat{U}, \partial_{\mathrm{v}} \widehat{U} ; \mathbb{R}\right) \rightarrow Z(\hat{\lambda}$, slits; $\mathbb{R})$ by the property that $\psi(c)=\alpha_{c}$ for every $c \in H_{1}\left(\widehat{U}, \partial_{\mathrm{v}} \widehat{U} ; \mathbb{R}\right)$.

Step 3. For every $c \in H_{1}\left(\widehat{U}, \partial_{\mathrm{v}} \widehat{U} ; \mathbb{R}\right)$ and every slit $s$ of $\hat{\lambda}, \partial \psi(c)(s) \in \mathbb{R}$ is the weight associated by $\partial c \in H_{0}\left(\partial_{\mathrm{v}} \widehat{U} ; \mathbb{R}\right)$ with the component $k_{s}$ of $\partial_{\mathrm{v}} \widehat{U}$ facing $s$.

This is just a rephrasing of the property that $\partial \alpha_{c}(s)=-\left[k_{s}\right] \cdot c$.

Step 4. The maps

$$
\varphi: Z(\hat{\lambda}, \text { slits } ; \mathbb{R}) \longrightarrow H_{1}\left(\widehat{U}, \partial_{\mathrm{v}} \widehat{U} ; \mathbb{R}\right) \quad \text { and } \quad \psi: H_{1}\left(\widehat{U}, \partial_{\mathrm{v}} \widehat{U} ; \mathbb{R}\right) \longrightarrow z(\hat{\lambda}, \text { slits } ; \mathbb{R})
$$

are inverse of each other. 
Pick a generic tie $k_{e}$ in each branch $e$ of $\widehat{U}$. By construction, the image $c=\varphi(\alpha)$ of $\alpha \in Z(\hat{\lambda}$, slits; $\mathbb{R})$ is defined by the property that $\alpha\left(k_{e}\right)=\left[k_{e}\right] \cdot c$ for every branch $e$. Conversely, for every $c \in H_{1}\left(\widehat{U}, \partial_{\mathrm{v}} \widehat{U} ; \mathbb{R}\right), \alpha=\psi(c)$ is characterized by the fact that $\alpha(k)=[k] \cdot c$ for every arc $k$ tightly transverse to $\hat{\lambda}$.

In particular, $\left[k_{e}\right] \cdot \varphi(\psi(c))=\left[k_{e}\right] \cdot c$ for every branch $e$, and it follows that $\varphi(\psi(c))=c$ in $H_{1}\left(\widehat{U}, \partial_{\mathrm{v}} \widehat{U} ; \mathbb{R}\right)$ by Poincaré-Lefschetz duality, since the $\left[k_{e}\right]$ generate $H_{1}\left(\widehat{U}, \partial_{\mathrm{h}} \widehat{U} ; \mathbb{R}\right)$. This proves that $\varphi \circ \psi$ is equal to the identity.

Conversely, for a relative tangent cycle $\alpha \in Z(\hat{\lambda}$, slits; $\mathbb{R})$, the same argument shows that $\psi(\varphi(\alpha))\left(k_{e}\right)=\alpha\left(k_{e}\right)$ for every branch $e$ of $\widehat{U}$. For a slit $s$, let $k_{s}$ be the component of $\partial_{\mathrm{v}} \widehat{U}$ that faces $s$, let $e$ be the branch of $\widehat{U}$ that contains $k_{s}$, and let $e_{1}$ and $e_{2}$ be the other two branches that touch $k_{s}$. Then, by quasi-additivity of $\alpha$ and $\psi(\varphi(\alpha)) \in \mathcal{Z}(\hat{\lambda}$, slits; $\mathbb{R})$,

$$
\begin{aligned}
\varepsilon(s) \partial \alpha(s) & =\alpha\left(k_{e_{1}}\right)+\alpha\left(k_{e_{2}}\right)-\alpha\left(k_{e}\right)=\psi(\varphi(\alpha))\left(k_{e_{1}}\right)+\psi(\varphi(\alpha))\left(k_{e_{2}}\right)-\psi(\varphi(\alpha))\left(k_{e}\right) \\
& =\varepsilon(s) \partial \psi(\varphi(\alpha))(s) .
\end{aligned}
$$

This proves that $\psi(\varphi(\alpha))-\alpha$ has boundary zero, and is therefore a tangent cycle by Lemma 4.4. Since $\psi(\varphi(\alpha))\left(k_{e}\right)-\alpha\left(k_{e}\right)=0$ for every branch $e$ of $\widehat{U}$, it follows from Proposition 4.2 that $\psi(\varphi(\alpha))-\alpha=0$.

This proves that $\psi \circ \varphi$ is the identity, and completes the proof of Proposition 4.5.

\subsection{Twisted relative tangent cycles}

So far, we have considered relative tangent cycles valued in $\mathbb{R}$. In our analysis of Hitchin characters, we will encounter relative tangent cycles that are valued in $\mathbb{R}^{n-1}$ and behave in a very specific manner with respect to the involution $\iota: \widehat{U} \rightarrow \widehat{U}$ that exchanges the two sheets of the cover $\widehat{U} \rightarrow U$.

More precisely, an $\mathbb{R}^{n-1}$-valued tangent cycle for $\hat{\lambda}$ relative to its slits associates a vector $\alpha(k) \in \mathbb{R}^{n-1}$ with each arc $k$ tightly transverse to $\hat{\lambda}$, in such a way that $\alpha$ is invariant under homotopy respecting $\hat{\lambda}$ and is quasi-additive with respect to a boundary function $\partial \alpha$ : slits of $\hat{\lambda}\} \rightarrow \mathbb{R}^{n-1}$.

A twisted tangent cycle for $\lambda$ relative to its slits and valued in $\widehat{\mathbb{R}}^{n-1}$ is an $\mathbb{R}^{n-1}$ valued relative tangent cycle $\alpha$ for $\hat{\lambda}$ such that, for every tightly transverse arc $k$,

$$
\alpha(\iota(k))=\overline{\alpha(k)},
$$

where $x \mapsto \bar{x}$ is the involution of $\mathbb{R}^{n-1}$ that reverses the order of the coordinates, namely that associates $\bar{x}=\left(x_{n-1}, x_{n-2}, \ldots, x_{1}\right)$ to $x=\left(x_{1}, x_{2}, \ldots, x_{n-1}\right) \in \mathbb{R}^{n-1}$. Let

$$
Z\left(\lambda, \text { slits } ; \widehat{\mathbb{R}}^{n-1}\right)=\left\{\alpha \in \mathcal{Z}\left(\hat{\lambda}, \text { slits } ; \mathbb{R}^{n-1}\right): \alpha(\iota(k))=\overline{\alpha(k)} \text { for all } k\right\}
$$


denote the space of these twisted relative tangent cycles.

The terminology and notation is justified by the fact that these twisted relative tangent cycles can be interpreted as tangent cycles for the geodesic lamination $\lambda$, relative to the slits of $\lambda$, and valued in the twisted coefficient bundle $\widehat{\mathbb{R}}^{n-1}=\left(\widehat{U} \times \mathbb{R}^{n-1}\right) / \mathbb{Z}_{2}$ over $U$, where $\mathbb{Z}_{2}$ acts by $\iota$ on $\widehat{U}$ and by $x \mapsto \bar{x}$ on $\mathbb{R}^{n-1}$.

We can similarly define the space of twisted tangent cycles $Z\left(\lambda ; \widehat{\mathbb{R}}^{n-1}\right)=\left\{\alpha \in \mathcal{Z}\left(\hat{\lambda} ; \mathbb{R}^{n-1}\right): \alpha(\iota(k))=\overline{\alpha(k)}\right.$ for all $\left.k\right\}=\left\{\alpha \in \mathcal{Z}\left(\lambda\right.\right.$, slits $\left.\left.; \widehat{\mathbb{R}}^{n-1}\right): \partial \alpha=0\right\}$, where the second equality comes from Lemma 4.4 .

To compute the dimensions of $z\left(\lambda ; \widehat{\mathbb{R}}^{n-1}\right)$ and $z\left(\lambda\right.$, slits; $\left.\widehat{\mathbb{R}}^{n-1}\right)$, we use a version of Propositions 4.2 and 4.5 that gives a homological interpretation of twisted tangent cycles. Because the orientation of arcs is irrelevant for relative tangent cycles but does matter in homology, we need to consider a different coefficient bundle $\widetilde{\mathbb{R}}^{n-1}=\left(\widehat{U} \times \mathbb{R}^{n-1}\right) / \mathbb{Z}_{2}$, where $\mathbb{Z}_{2}$ still acts by the covering involution $\iota$ on $\widehat{U}$ but now acts on $\mathbb{R}^{n-1}$ by $x \mapsto-\bar{x}$.

More precisely, because $\iota$ reverses the orientation of $\hat{\lambda}$, the map

$$
\varphi: Z(\hat{\lambda}, \text { slits } ; \mathbb{R}) \longrightarrow H_{1}\left(\widehat{U}, \partial_{\mathrm{v}} \widehat{U} ; \mathbb{R}\right)
$$

of the proof of Proposition 4.5 conjugates the action of $\iota$ on $Z(\hat{\lambda}$, slits; $\mathbb{R})$ to $-\iota_{*}$, where $\iota_{*}: H_{1}\left(\widehat{U}, \partial_{\mathrm{v}} \widehat{U} ; \mathbb{R}\right) \rightarrow H_{1}\left(\widehat{U}, \partial_{\mathrm{v}} \widehat{U} ; \mathbb{R}\right)$ is the homomorphism induced by $\iota$. Therefore, the tensor product $\varphi \otimes \operatorname{Id}_{\mathbb{R}^{n-1}}$ sends $Z\left(\lambda\right.$, slits; $\left.\widehat{\mathbb{R}}^{n-1}\right)$ to $\left\{c \in H_{1}\left(\widehat{U}, \partial_{\mathrm{v}} \widehat{U} ; \mathbb{R}^{n-1}\right) ; \iota_{*}(c)=-\bar{c}\right\}$, which is naturally identified to $H_{1}\left(U, \partial_{\mathrm{v}} U ; \widetilde{\mathbb{R}}^{n-1}\right)$. This provides a natural isomorphism $\mathcal{Z}\left(\lambda\right.$, slits; $\left.\widehat{\mathbb{R}}^{n-1}\right) \cong H_{1}\left(U, \partial_{\mathrm{v}} U ; \widetilde{\mathbb{R}}^{n-1}\right)$, which also induces an isomorphism

$$
z\left(\lambda ; \widehat{\mathbb{R}}^{n-1}\right) \cong H_{1}\left(U ; \widetilde{\mathbb{R}}^{n-1}\right) .
$$

Proposition 4.6. The above construction provides natural isomorphisms

$$
\begin{aligned}
& z\left(\lambda, \text { slits } ; \widehat{\mathbb{R}}^{n-1}\right) \cong H_{1}\left(U, \partial_{\mathrm{v}} U ; \widetilde{\mathbb{R}}^{n-1}\right), \\
& z\left(\lambda ; \widehat{\mathbb{R}}^{n-1}\right) \cong H_{1}\left(U ; \widetilde{\mathbb{R}}^{n-1}\right) .
\end{aligned}
$$

Proof. This follows from the above discussion.

Proposition 4.7. The vector spaces $Z\left(\lambda ; \widehat{\mathbb{R}}^{n-1}\right)$ and $Z\left(\lambda\right.$, slits; $\left.\widehat{\mathbb{R}}^{n-1}\right)$ have dimensions

$$
\begin{aligned}
\operatorname{dim} z\left(\lambda ; \widehat{\mathbb{R}}^{n-1}\right) & =6(g-1)(n-1)+\left\lfloor\frac{1}{2}(n-1)\right\rfloor, \\
\operatorname{dim} Z\left(\lambda, \operatorname{slits} ; \widehat{\mathbb{R}}^{n-1}\right) & =18(g-1)(n-1),
\end{aligned}
$$

where $\lfloor x\rfloor$ denotes the largest integer that is less than or equal to $x$. 
Proof. Considering Euler characteristics,

$$
\chi(U)(n-1)=\operatorname{dim} H_{0}\left(U ; \widetilde{\mathbb{R}}^{n-1}\right)-\operatorname{dim} H_{1}\left(U ; \widetilde{\mathbb{R}}^{n-1}\right) .
$$

Since $\widehat{U}$ is connected,

$$
H_{0}\left(U ; \widetilde{\mathbb{R}}^{n-1}\right)=\left\{c \in H_{0}\left(\widehat{U} ; \mathbb{R}^{n-1}\right): \iota_{*}(c)=-\bar{c}\right\} \cong\left\{x \in \mathbb{R}^{n-1}: x=-\bar{x}\right\}
$$

has dimension $\left\lfloor\frac{1}{2}(n-1)\right\rfloor$. Moreover, because the complement $S \backslash U$ consists of $4(g-1)$ hexagons, $\chi(U)=\chi(S)-4(g-1)=-6(g-1)$. It follows that

$$
\begin{aligned}
\operatorname{dim} z\left(\lambda ; \widehat{\mathbb{R}}^{n-1}\right) & =\operatorname{dim} H_{1}\left(U ; \widetilde{\mathbb{R}}^{n-1}\right)=-\chi(U)(n-1)+\operatorname{dim} H_{0}\left(U ; \widetilde{\mathbb{R}}^{n-1}\right) \\
& =6(g-1)(n-1)+\left\lfloor\frac{1}{2}(n-1)\right\rfloor .
\end{aligned}
$$

For $Z\left(\lambda\right.$, slits $\left.; \widehat{\mathbb{R}}^{n-1}\right) \cong H_{1}\left(U, \partial_{\mathrm{v}} U ; \widetilde{\mathbb{R}}^{n-1}\right)$, consider the exact sequence

$$
0 \longrightarrow H_{1}\left(U ; \widetilde{\mathbb{R}}^{n-1}\right) \longrightarrow H_{1}\left(U, \partial_{\mathrm{v}} U ; \widetilde{\mathbb{R}}^{n-1}\right) \longrightarrow H_{0}\left(\partial_{\mathrm{v}} U ; \widetilde{\mathbb{R}}^{n-1}\right) \longrightarrow H_{0}\left(U ; \widetilde{\mathbb{R}}^{n-1}\right) \longrightarrow 0 .
$$

We already observed that $\operatorname{dim} H_{0}\left(U ; \widetilde{\mathbb{R}}^{n-1}\right)=\left\lfloor\frac{1}{2}(n-1)\right\rfloor$. Since $\iota$ respects no component of $\partial_{\mathrm{v}} \widehat{U}$, the twisted homology space $H_{0}\left(\partial_{\mathrm{v}} U ; \widetilde{\mathbb{R}}^{n-1}\right)$ is isomorphic to $H_{0}\left(\partial_{\mathrm{v}} U ; \mathbb{R}^{n-1}\right)$ and therefore has dimension $12(g-1)(n-1)$, as $\partial_{\mathrm{v}} U$ has $12(g-1)$ components. It follows from the exact sequence above that

$$
\begin{aligned}
\operatorname{dim} Z\left(\lambda, \operatorname{sits} ; \widehat{\mathbb{R}}^{n-1}\right) & =\operatorname{dim} H_{1}\left(U, \partial_{\mathrm{v}} U ; \widetilde{\mathbb{R}}^{n-1}\right) \\
& =\operatorname{dim} H_{1}\left(U ; \widetilde{\mathbb{R}}^{n-1}\right)+\operatorname{dim} H_{0}\left(\partial_{\mathrm{v}} U ; \widetilde{\mathbb{R}}^{n-1}\right)-\operatorname{dim} H_{0}\left(U ; \widetilde{\mathbb{R}}^{n-1}\right) \\
& =18(g-1)(n-1) .
\end{aligned}
$$

As suggested by the formula and the fact that $U$ has $18(g-1)$ branches, there is a more elementary (non-homological) computation which deduces the dimension of $Z\left(\lambda\right.$, slits; $\left.\widehat{\mathbb{R}}^{n-1}\right)$ from Proposition 4.5 , but this one is more intrinsic and conceptual.

\subsection{Relative tangent cycles from another viewpoint}

We give a different description of relative tangent cycles. Compared to the original definition, this presentation does not lend itself as well to the homological interpretation and computations of the previous sections. However, it will be better adapted to the geometric constructions that form the core of this article. It also bypasses the need to consider the orientation cover $\hat{\lambda}$.

In the universal cover $\widetilde{S}$ of $S$, let $\widetilde{U}$ be the preimage of a train-track neighborhood $U$ of $\lambda$. 
A relative tangent cycle $\alpha \in \mathcal{Z}(\hat{\lambda}$, slits; $\mathbb{R})$ associates a number $\alpha\left(T, T^{\prime}\right) \in \mathbb{R}$ with each ordered pair of distinct components $T$ and $T^{\prime}$ of $\widetilde{S} \backslash \tilde{\lambda}$ as follows. Choose an oriented arc $\tilde{k} \subset \widetilde{S}$ that is tightly transverse to $\tilde{\lambda}$ and joins $T$ to $T^{\prime}$; in this preliminary stage, one can for instance take for $\tilde{k}$ any geodesic arc going from $T$ to $T^{\prime}$, since every component of $\widetilde{S} \backslash \tilde{\lambda}$ is a triangle. Using Proposition 4.1, one can modify $\tilde{k}$ by a homotopy respecting $\tilde{\lambda}$ so that it is contained in $\widetilde{U}$, and is tightly transverse to $\tilde{\lambda}$ in $\widetilde{U}$. Project $\tilde{k}$ to an $\operatorname{arc}$ $k \subset U$, which is tightly transverse to $\lambda$.

The tightly transverse arc $k$ admits two lifts to the 2-fold cover $\widehat{U}$ of $U$, each oriented so that the canonical orientation of the leaves of the orientation cover $\hat{\lambda}$ points to the left of these arcs at each intersection point. Let $\hat{k} \subset \widehat{U}$ be the lift whose orientation projects to the same orientation of $k$ as that of $\tilde{k}$. By construction, $\hat{k}$ is tightly transverse to $\hat{\lambda}$ in $\widehat{U}$, and we can consider the number $\alpha(\hat{k}) \in \mathbb{R}$ defined by $\alpha \in \mathcal{Z}(\hat{\lambda}$, slits; $\mathbb{R})$.

In this construction, the $\operatorname{arc} \tilde{k}$ is uniquely determined by $T$ and $T^{\prime}$ up to homotopy respecting $\tilde{\lambda}$ in $\widetilde{U}$, which determines $\hat{k}$ up to homotopy respecting $\hat{\lambda}$ in $\widehat{U}$. It follows that $\alpha(\hat{k})$ depends only on $T$ and $T^{\prime}$, and we can define $\alpha\left(T, T^{\prime}\right)=\alpha(\hat{k}) \in \mathbb{R}$.

The quasi-additivity property of $\alpha \in \mathcal{Z}(\hat{\lambda}$, slits; $\mathbb{R})$ has a relatively simple translation in this context. Each slit $s$ of $\lambda$, namely each spike of $S \backslash \lambda$, lifts to two slits of $\hat{\lambda}$ : a positive spike $s^{+}$of $\widehat{U} \backslash \hat{\lambda}$ where the leaves of $\hat{\lambda}$ adjacent to $s^{+}$are oriented towards the end of this spike by the canonical orientation of $\hat{\lambda}$, and a negative spike $s^{-}$where the adjacent leaves are oriented away from the end of $s^{-}$. Define two functions $\partial^{+} \alpha, \partial^{-} \alpha$ : slits of $\left.\lambda\right\} \rightarrow \mathbb{R}$ by the property that $\partial^{+} \alpha(s)=\partial \alpha\left(s^{+}\right)$and $\partial^{-} \alpha(s)=\partial \alpha\left(s^{-}\right)$for every slit $s$ of $\lambda$, where $\partial \alpha:\{$ slits of $\hat{\lambda}\} \rightarrow \mathbb{R}$ is the boundary of $\alpha \in \mathcal{Z}(\hat{\lambda}$, slits; $\mathbb{R})$.

If $T, T^{\prime}$ and $T^{\prime \prime}$ are three components of $\widetilde{S} \backslash \tilde{\lambda}$ such that $T^{\prime \prime}$ separates $T$ from $T^{\prime}$ in $\widetilde{S}$, let $\tilde{s}^{\prime \prime}$ be the spike of $T^{\prime \prime}$ delimited by the two sides of $T^{\prime \prime}$ that separate $T$ from $T^{\prime}$, and let $s^{\prime \prime}$ be the projection of $\tilde{s}^{\prime \prime}$ to $S$. The quasi-additivity of $\alpha \in \mathcal{Z}(\hat{\lambda}$, slits; $\mathbb{R})$ then translates to the property that

$$
\alpha\left(T, T^{\prime}\right)=\alpha\left(T, T^{\prime \prime}\right)+\alpha\left(T^{\prime \prime}, T^{\prime}\right)-\partial^{+} \alpha\left(s^{\prime \prime}\right)
$$

if the spike $\tilde{s}^{\prime \prime}$ of $T^{\prime \prime}$ points to the left as seen from $T$, and

$$
\alpha\left(T, T^{\prime}\right)=\alpha\left(T, T^{\prime \prime}\right)+\alpha\left(T^{\prime \prime}, T^{\prime}\right)+\partial^{-} \alpha\left(s^{\prime \prime}\right)
$$

if $\tilde{s}^{\prime \prime}$ points to the right as seen from $T$.

Proposition 4.8. The above construction provides a one-to-one correspondence between relative tangent cycles $\alpha \in \mathcal{Z}(\hat{\lambda}$, slits; $\mathbb{R})$ and maps $\alpha$ associating a number $\alpha\left(T, T^{\prime}\right) \in$ $\mathbb{R}$ with each ordered pair of distinct components $T$ and $T^{\prime}$ of $\widetilde{S} \backslash \tilde{\lambda}$ for which there exist two functions $\partial^{ \pm} \alpha$ : $\{$ slits of $\lambda\} \rightarrow \mathbb{R}$ with the following properties:

(1) $\alpha$ is $\pi_{1}(S)$-invariant, in the sense that $\alpha\left(\gamma T, \gamma T^{\prime}\right)=\alpha\left(T, T^{\prime}\right)$ for every $\gamma \in \pi_{1}(S)$ and every pair of distinct components $T$ and $T^{\prime}$ of $\widetilde{S} \backslash \tilde{\lambda}$; 
(2) if $T^{\prime \prime}$ separates $T$ from $T^{\prime}$ in $\widetilde{S}$, if $\tilde{s}^{\prime \prime}$ is the spike of $T^{\prime \prime}$ delimited by the two sides of $T^{\prime \prime}$ that separate $T$ from $T^{\prime}$, and if $s^{\prime \prime}$ is the slit of $\lambda$ defined by the projection of $\tilde{s}^{\prime \prime}$ to $S$, then

$$
\alpha\left(T, T^{\prime}\right)=\alpha\left(T, T^{\prime \prime}\right)+\alpha\left(T^{\prime \prime}, T^{\prime}\right)-\partial^{+} \alpha\left(s^{\prime \prime}\right)
$$

if $\tilde{s}^{\prime \prime}$ points to the left as seen from $T$, and

$$
\alpha\left(T, T^{\prime}\right)=\alpha\left(T, T^{\prime \prime}\right)+\alpha\left(T^{\prime \prime}, T^{\prime}\right)+\partial^{-} \alpha\left(s^{\prime \prime}\right)
$$

if $\tilde{s}^{\prime \prime}$ points to the right as seen from $T$.

In addition, the boundary $\partial \alpha:\{$ slits of $\hat{\lambda}\} \rightarrow \mathbb{R}$ is related to the functions

$$
\partial^{ \pm} \alpha:\{\text { slits of } \lambda\} \longrightarrow \mathbb{R}
$$

by the property that $\partial \alpha\left(s^{ \pm}\right)=\partial^{ \pm} \alpha(s)$ for every slit $s$ of $\lambda$ lifting to a positive slit $s^{+}$and a negative slit $s^{-}$of the orientation cover $\hat{\lambda}$.

Proof. This is an immediate consequence of the discussion above.

Proposition 4.8 has an immediate factor-by-factor extension to relative tangent cycles valued in $\mathbb{R}^{n-1}$. By restriction to the space of twisted relative tangent cycles $z\left(\lambda\right.$, slits; $\left.\widehat{\mathbb{R}}^{n-1}\right) \subset Z\left(\hat{\lambda}\right.$, slits; $\left.\mathbb{R}^{n-1}\right)$, this automatically gives the following statement. Recall that $x \mapsto \bar{x}$ denotes the involution of $\mathbb{R}^{n-1}$ that sends $x=\left(x_{1}, x_{2}, \ldots, x_{n-1}\right)$ to $\bar{x}=\left(x_{n-1}, x_{n-2}, \ldots, x_{1}\right)$.

Proposition 4.9. Proposition 4.8 provides a one-to-one correspondence between twisted relative tangent cycles $\alpha \in Z\left(\lambda\right.$, slits; $\left.\widehat{\mathbb{R}}^{n-1}\right)$ and maps $\alpha$ which associate a vector $\alpha\left(T, T^{\prime}\right) \in \mathbb{R}^{n-1}$ with each ordered pair of components $T$ and $T^{\prime}$ of $\widetilde{S} \backslash \tilde{\lambda}$ such that there exists a function $\partial^{+} \alpha:\{$ slits of $\lambda\} \rightarrow \mathbb{R}^{n-1}$ with the following properties:

(1) $\alpha$ is $\pi_{1}(S)$-equivariant, in the sense that $\alpha\left(\gamma T, \gamma T^{\prime}\right)=\alpha\left(T, T^{\prime}\right)$ for each $\gamma \in \pi_{1}(S)$ and each pair of distinct components $T$ and $T^{\prime}$ of $\widetilde{S} \backslash \tilde{\lambda}$;

(2) if $T^{\prime \prime}$ separates $T$ from $T^{\prime}$ in $\widetilde{S}$, if $\tilde{s}^{\prime \prime}$ is the spike of $T^{\prime \prime}$ delimited by the two sides of $T^{\prime \prime}$ that separate $T$ from $T^{\prime}$, and if $s^{\prime \prime}$ is the slit of $\lambda$ defined by the projection of $\tilde{s}^{\prime \prime}$ to $S$, then

$$
\alpha\left(T, T^{\prime}\right)=\alpha\left(T, T^{\prime \prime}\right)+\alpha\left(T^{\prime \prime}, T^{\prime}\right)-\partial^{+} \alpha\left(s^{\prime \prime}\right)
$$

if $\tilde{s}^{\prime \prime}$ points to the left as seen from $T$, and

$$
\alpha\left(T, T^{\prime}\right)=\alpha\left(T, T^{\prime \prime}\right)+\alpha\left(T^{\prime \prime}, T^{\prime}\right)-\overline{\partial^{+} \alpha\left(s^{\prime \prime}\right)}
$$

if $\tilde{s}^{\prime \prime}$ points to the right as seen from $T$;

(3) $\alpha\left(T^{\prime}, T\right)=\overline{\alpha\left(T, T^{\prime}\right)}$ for every pair of distinct components $T$ and $T^{\prime}$ of $\widetilde{S} \backslash \tilde{\lambda}$. 
In addition, the boundary $\partial \alpha:\{$ slits of $\hat{\lambda}\} \rightarrow \mathbb{R}$ is related to the function

$$
\partial^{+} \alpha:\{\text { slits of } \lambda\} \longrightarrow \mathbb{R}
$$

by the property that $\partial \alpha\left(s^{+}\right)=\partial^{+} \alpha(s)$ and $\partial \alpha\left(s^{-}\right)=-\overline{\partial^{+} \alpha(s)}$ for every slit $s$ of $\lambda$ lifting to a positive slit $s^{+}$and a negative slit $s^{-}$of the orientation cover $\tilde{\lambda}$.

Proof. This statement immediately follows from Proposition 4.8.

Note that the function $\partial^{-} \alpha$ : slits of $\left.\lambda\right\} \rightarrow \mathbb{R}^{n-1}$ that one would have expected in this case is equal to $\partial^{-} \alpha=-\overline{\partial^{+} \alpha}$ by the third condition of Proposition 4.9. In particular, $\partial \alpha\left(s^{\mp}\right)=-\overline{\partial \alpha\left(s^{ \pm}\right)}$for every $\alpha \in \mathcal{Z}\left(\lambda\right.$, slits; $\left.\widehat{\mathbb{R}}^{n-1}\right)$, when $s^{+}$and $s^{-}$are the positive and negative slits of the orientation cover $\hat{\lambda}$ that lift the same slit $s$ of $\lambda$.

\section{The shearing tangent cycle of a Hitchin character}

We will now associate a twisted relative tangent cycle

$$
\sigma^{\varrho} \in \mathcal{Z}\left(\lambda, \text { slits; } \widehat{\mathbb{R}}^{n-1}\right) \cong H_{1}\left(U, \partial_{\mathrm{v}} U ; \widetilde{\mathbb{R}}^{n-1}\right)
$$

with each Hitchin character $\varrho \in \operatorname{Hit}_{n}(S)$. The key ingredient of this construction is the slithering map introduced in the next section.

\subsection{Slithering}

The slithering construction is a higher-dimensional analogue of the horocyclic foliation defined, in the case where $n=2$, by a hyperbolic metric and a maximal geodesic lamination $\lambda$ on the surface $S$; see [42] and [5] for that case. It only uses the Anosov property of Theorem 3.1 and Proposition 3.2, and is independent of the positivity property of Theorem 3.3. In particular, it quite possibly extends to more general Anosov contexts, such as those of [22].

Consider a Hitchin homomorphism $\varrho: \pi_{1}(S) \rightarrow \mathrm{PSL}_{n}(\mathbb{R})$, and its associated flag map $\mathcal{F}_{\varrho}: \partial_{\infty} \widetilde{S} \rightarrow \operatorname{Flag}\left(\mathbb{R}^{n}\right)$ as in $\S 3.1$.

In the universal cover $\widetilde{S}$, let $g$ be a leaf of the preimage $\tilde{\lambda} \subset \widetilde{S}$ of the maximal geodesic lamination $\lambda \subset S$. Choose an arbitrary orientation for $g$, and let $x_{+}$and $x_{-}$be its positive and negative endpoints, respectively. By Theorem 3.3, the flag pair $\left(\mathcal{F}_{\varrho}\left(x_{+}\right), \mathcal{F}_{\varrho}\left(x_{-}\right)\right)$ is generic. It therefore defines a decomposition of $\mathbb{R}^{n}$ as the direct sum of the lines $\tilde{L}_{a}(g)=\mathcal{F}_{\varrho}\left(x_{+}\right)^{(a)} \cap \mathcal{F}_{\varrho}\left(x_{-}\right)^{(n-a+1)}$, as in $\S 3.1$.

Note that reversing the orientation of $g$ exchanges $x_{+}$and $x_{-}$, and therefore replaces $\tilde{L}_{a}(g)$ by $\tilde{L}_{n-a+1}(g)$. 
Now consider two leaves $g$ and $g^{\prime} \subset \tilde{\lambda}$. We say that $g$ and $g^{\prime}$ are oriented in parallel if exactly one of the orientations of $g$ and $g^{\prime}$ coincides with the boundary orientation determined by the component of $S \backslash\left(g \cup g^{\prime}\right)$ that separates $g$ from $g^{\prime}$.

Proposition 5.1. There exists a unique family of linear isomorphisms

$$
\Sigma_{g g^{\prime}}: \mathbb{R}^{n} \longrightarrow \mathbb{R}^{n}
$$

indexed by all pairs of leaves $g, g^{\prime} \subset \tilde{\lambda}$, such that the following conditions hold:

(1) $\Sigma_{g g}=\operatorname{Id}_{\mathbb{R}^{n}}, \Sigma_{g^{\prime} g}=\left(\Sigma_{g g^{\prime}}\right)^{-1}$ and $\Sigma_{g g^{\prime \prime}}=\Sigma_{g g^{\prime}} \circ \Sigma_{g^{\prime} g^{\prime \prime}}$ when $g^{\prime}$ separates $g$ from $g^{\prime \prime}$;

(2) $\Sigma_{g g^{\prime}}$ depends locally Hölder continuously on $g$ and $g^{\prime}$; namely, for an arbitrary riemannian metric on the manifold consisting of all geodesics of the universal cover $\widetilde{S}$, the map $\left(g, g^{\prime}\right) \mapsto \Sigma_{g g^{\prime}}$ is Hölder continuous on (the square of) any compact subset of the space of leaves of $\tilde{\lambda}$;

(3) if $g$ and $g^{\prime}$ have an endpoint $x \in \partial_{\infty} \widetilde{S}$ in common and are oriented towards $x$, and if $E=\mathcal{F}_{\varrho}(x) \in \operatorname{Flag}\left(\mathbb{R}^{n}\right)$, then $\Sigma_{g g^{\prime}}$ sends each line $\tilde{L}_{a}\left(g^{\prime}\right)$ to $\tilde{L}_{a}(g)$ and its restriction $\tilde{L}_{a}\left(g^{\prime}\right) \rightarrow \tilde{L}_{a}(g)$ is the composition of the two natural isomorphisms

$$
\tilde{L}_{a}\left(g^{\prime}\right) \cong E^{(a)} / E^{(a-1)} \cong \tilde{L}_{a}(g) .
$$

In addition, the maps $\Sigma_{g g^{\prime}}$ satisfy:

(4) if $g$ and $g^{\prime}$ are oriented in parallel, $\Sigma_{g g^{\prime}}$ sends each line $\tilde{L}_{a}\left(g^{\prime}\right)$ to the line $\tilde{L}_{a}(g)$;

(5) $\Sigma_{g g^{\prime}}: \mathbb{R}^{n} \rightarrow \mathbb{R}^{n}$ has determinant 1 .

By definition, $\Sigma_{g g^{\prime}}: \mathbb{R}^{n} \rightarrow \mathbb{R}^{n}$ is the slithering map from the line decomposition

$$
\mathbb{R}^{n}=\bigoplus_{a=1}^{n} \tilde{L}_{a}\left(g^{\prime}\right)
$$

to the line decomposition

$$
\mathbb{R}^{n}=\bigoplus_{a=1}^{n} \tilde{L}_{a}(g)
$$

We will construct $\Sigma_{g g^{\prime}}$ by sweeping through all the leaves of $\tilde{\lambda}$ that separate $g$ from $g^{\prime}$, and by composition of a (usually infinite) sequence of pivot moves as in condition (3) of Proposition 5.1. The terminology of "slithering" is motivated by the fact that, in general, any small section of this sweep involves both pivot moves to the left and pivot moves to the right. $\left({ }^{1}\right)$

Note that, although the line decomposition $\mathbb{R}^{n}=\bigoplus_{a=1}^{n} \tilde{L}_{a}(g)$ depends on an orientation for the leaf $g$, the slithering map $\Sigma_{g g^{\prime}}: \mathbb{R}^{n} \rightarrow \mathbb{R}^{n}$ is independent of a choice of orientation for $g$ or $g^{\prime}$.

( ${ }^{1}$ In particular, this is unrelated to Thurston's notion [41], [12] of "slithering" for foliations of 3-dimensional manifolds, beyond the analogy with the movements of a snake. 
Proof of Proposition 5.1. We will split the construction of the slithering map of Proposition 5.1 into several steps and lemmas.

Let $T$ be a component of $\widetilde{S} \backslash \tilde{\lambda}$ that separates the leaves $g$ and $g^{\prime}$ of $\tilde{\lambda}$. It is a triangle since the geodesic lamination $\lambda$ is maximal, and two of its three sides separate $g$ from $g^{\prime}$; among these two sides, let $g_{T}$ be the one that is closest to $g$, and $g_{T}^{\prime}$ the one closest to $g^{\prime}$. Define $\Sigma_{T}=\Sigma_{g_{T} g_{T}^{\prime}}$ by condition (3) of Proposition 5.1. Namely, if $E_{T}=\mathcal{F}_{\varrho}\left(x_{T}\right) \in \operatorname{Flag}\left(\mathbb{R}^{n}\right)$ is the image under the flag map $\mathcal{F}_{\varrho}: \partial_{\infty} \widetilde{S} \rightarrow \operatorname{Flag}\left(\mathbb{R}^{n}\right)$ of the common endpoint $x_{T} \in \partial_{\infty} \widetilde{S}$ of $g_{T}$ and $g_{T}^{\prime}$, the map $\Sigma_{T}=\Sigma_{g_{T} g_{T}^{\prime}}$ sends $\mathbb{R}^{n}=\bigoplus_{a=1}^{n} \tilde{L}_{a}\left(g_{T}^{\prime}\right)$ to $\mathbb{R}^{n}=\bigoplus_{a=1}^{n} \tilde{L}_{a}\left(g_{T}\right)$ by the property that its restriction $\tilde{L}_{a}\left(g_{T}^{\prime}\right) \rightarrow \tilde{L}_{a}\left(g_{T}\right)$ coincides with the composition of the natural isomorphisms $\tilde{L}_{a}\left(g_{T}^{\prime}\right) \cong E_{T}^{(a)} / E_{T}^{(a-1)} \cong \tilde{L}_{a}\left(g_{T}\right)$. Note that, in a basis of $\mathbb{R}^{n}$ where the $a$ th vector belongs to $\tilde{L}_{a}\left(g_{T}^{\prime}\right)$, the map $\Sigma_{T}$ is represented by a triangular matrix with all diagonal entries equal to 1 . In particular, $\tilde{L}_{a}\left(g_{T}^{\prime}\right)$ has determinant 1 and therefore belongs to $\mathrm{SL}_{n}(\mathbb{R})$.

We will now define

$$
\Sigma_{g g^{\prime}}=\prod_{T} \Sigma_{T}
$$

as the composition of the maps $\Sigma_{T}=\Sigma_{g_{T} g_{T}^{\prime}}: \mathbb{R}^{n} \rightarrow \mathbb{R}^{n}$ as $T$ ranges over all components of $\widetilde{S} \backslash \tilde{\lambda}$ separating $g$ from $g^{\prime}$. Of course, there usually are infinitely many maps in this composition, and we also must be careful with the order in which we compose these maps; the arrow over the product symbol is here to remind us that this is an ordered product, if the components $T$ are ordered from $g$ to $g^{\prime}$. To make sense of this composition, let $\mathcal{T}_{g g^{\prime}}$ be the set of components of $\widetilde{S} \backslash \tilde{\lambda}$ that separate $g$ from $g^{\prime}$. Let $\mathcal{T}=\left\{T_{1}, T_{2}, \ldots, T_{m}\right\}$ be a finite subset of $\mathcal{T}_{g g^{\prime}}$, where the indexing is chosen so that each ideal triangle $T_{j}$ separates $g$ from $T_{j+1}$. We can then consider the finite composition

$$
\Sigma_{\mathcal{T}}=\Sigma_{T_{1}} \circ \Sigma_{T_{2}} \circ \ldots \circ \Sigma_{T_{m-1}} \circ \Sigma_{T_{m}} \in \mathrm{SL}_{n}(\mathbb{R}) .
$$

We will then show that $\Sigma_{\mathcal{T}}$ converges to some linear map $\Sigma_{g g^{\prime}} \in \mathrm{SL}_{n}(\mathbb{R})$ as the finite subset $\mathcal{T}=\left\{T_{1}, T_{2}, \ldots, T_{m}\right\}$ tends to the whole set $\mathcal{T}_{g g^{\prime}}$ of those components of $\widetilde{S} \backslash \tilde{\lambda}$ which separate $g$ from $g^{\prime}$.

The proof of convergence relies on the following estimate. Choose an $\operatorname{arc} k \subset \widetilde{S}$ that is tightly transverse to the geodesic lamination $\tilde{\lambda}$, and crosses both $g$ and $g^{\prime}$; for instance, we can choose $k$ to be a geodesic arc.

In particular, for every component $T$ of $\widetilde{S} \backslash \tilde{\lambda}$ that separates $g$ from $g^{\prime}, k \cap T$ consists of a single arc.

Endow the space $\operatorname{End}\left(\mathbb{R}^{n}\right)$ of linear maps $\mathbb{R}^{n} \rightarrow \mathbb{R}^{n}$ with any of the classical norms $\|\cdot\|$ such that $\left\|\operatorname{Id}_{\mathbb{R}^{n}}\right\|=1$ and $\|\varphi \circ \psi\| \leqslant\|\varphi\|\|\psi\|$. Our estimates will also depend on the choice of a negatively curved metric $m$ on $S$ for which the leaves of $\lambda$ are geodesic. 
Lemma 5.2. Given an arc $k$ as above, there exist constants $A$ and $\nu>0$ such that

$$
\left\|\Sigma_{T}-\operatorname{Id}_{\mathbb{R}^{n}}\right\| \leqslant A \ell(k \cap T)^{\nu}
$$

for every component $T$ of $\widetilde{S} \backslash \tilde{\lambda}$ that separates $g$ from $g^{\prime}$, where $\ell$ denotes the arc length for the auxiliary metric $m$.

Proof. Let $x_{T}, y_{T}, y_{T}^{\prime} \in \partial_{\infty} \widetilde{S}$ denote the three vertices of the triangle $T$, in such a way that $x_{T}$ and $y_{T}$ are the endpoints of the side $g_{T}$ that is closest to $g$, and $x_{T}$ and $y_{T}^{\prime}$ are the endpoints of the side $g_{T}^{\prime}$ closest to $g^{\prime}$. Then $\Sigma_{T}=\Sigma_{g_{T} g_{T}^{\prime}}$ depends only on the two generic flag pairs $\left(\mathcal{F}_{\varrho}\left(x_{T}\right), \mathcal{F}_{\varrho}\left(y_{T}\right)\right)$ and $\left(\mathcal{F}_{\varrho}\left(x_{T}\right), \mathcal{F}_{\varrho}\left(y_{T}^{\prime}\right)\right)$. In fact, $\Sigma_{T}$ depends differentiably on these two flag pairs and, as $T$ varies, these pairs stay in a compact subset of the space of generic flag pairs (depending on $k$ and on the continuity of the flag curve $\mathcal{F}_{\varrho}$ ). Therefore,

$$
\left\|\Sigma_{T}-\operatorname{Id}_{\mathbb{R}^{n}}\right\|=O\left(d\left(\mathcal{F}_{\varrho}\left(y_{T}\right), \mathcal{F}_{\varrho}\left(y_{T}^{\prime}\right)\right)\right),
$$

where $d$ is an arbitrary riemannian metric on $\operatorname{Flag}\left(\mathbb{R}^{n}\right)$.

Since the flag curve $\mathcal{F}_{\varrho}$ is Hölder continuous (Proposition 3.2),

$$
d\left(\mathcal{F}_{\varrho}\left(y_{T}\right), \mathcal{F}_{\varrho}\left(y_{T}^{\prime}\right)\right)=O\left(d\left(y_{T}, y_{T}^{\prime}\right)^{\nu}\right)
$$

for some Hölder exponent $\nu$, where $d$ now denotes the visual metric of $\partial_{\infty} \widetilde{S}$. The required estimate then follows from an easy geometric argument in the triangle $T$ showing that

$$
d\left(y_{T}, y_{T}^{\prime}\right)=O(\ell(k \cap T)) .
$$

Note that the constant $A$ depends on the arc $k$. The Hölder exponent $\nu$ depends only on the flag curve $\mathcal{F}_{\varrho}$.

The second ingredient is a now classical property of geodesic laminations, based on the fundamental property that $S \backslash \lambda$ has only finitely many spikes and that the width of these spikes decay exponentially.

LEMMA 5.3. As $T$ ranges over all components of $\widetilde{S} \backslash \tilde{\lambda}$ separating $g$ from $g^{\prime}$, the sum

$$
\sum_{T \in \mathcal{T}_{g g^{\prime}}} \ell(k \cap T)^{\nu}
$$

is convergent for every $\nu>0$.

More precisely, there is a function $r: \mathcal{T}_{g g^{\prime}} \rightarrow \mathbb{N}$ and constants $B, C, B^{\prime}, C^{\prime}>0$ such that the following conditions hold:

(1) $B e^{-C r(T)} \leqslant \ell(k \cap T) \leqslant B^{\prime} e^{-C^{\prime} r(T)}$ for every $T \in \mathcal{T}_{g g^{\prime}}$;

(2) for every $m \in \mathbb{N}$, the number of triangles $T \in \mathcal{T}_{\text {gg' }}$ with $r(T)=m$ is uniformly bounded, independently of $m$. 
Proof. See for instance [5, Lemmas 4 and 5], and compare $§ 8.2$.

We are now ready to show the convergence of the infinite product $\vec{\prod}_{T} \Sigma_{T}$.

Recall that $\mathcal{T}_{g g^{\prime}}$ denotes the set of components of $\widetilde{S} \backslash \tilde{\lambda}$ that separate $g$ from $g^{\prime}$ and that, for every finite subset $\mathcal{T}=\left\{T_{1}, T_{2}, \ldots, T_{m}\right\}$ of $\mathcal{T}_{g g^{\prime}}$ where the $T_{i}$ are ordered from $g$ to $g^{\prime}$,

$$
\Sigma_{\mathcal{T}}=\Sigma_{T_{1}} \circ \Sigma_{T_{2}} \circ \ldots \circ \Sigma_{T_{m-1}} \circ \Sigma_{T_{m}} .
$$

Lemma 5.4. As $\mathcal{T}$ ranges over all finite subsets of $\mathcal{T}_{g g^{\prime}}$, the matrices $\Sigma_{\mathcal{T}}$ remain uniformly bounded.

Proof. If $\mathcal{T}=\left\{T_{1}, T_{2}, \ldots, T_{m}\right\}$, Lemma 5.2 shows that $\left\|\Sigma_{T_{i}}\right\| \leqslant 1+A \ell\left(k \cap T_{i}\right)^{\nu}$ for some constants $A, \nu>0$. Then,

$$
\left\|\Sigma_{\mathcal{T}}\right\| \leqslant \prod_{i=1}^{m}\left(1+A \ell\left(k \cap T_{i}\right)^{\nu}\right) \leqslant \prod_{T \in \mathcal{T}_{g g^{\prime}}}\left(1+A \ell(k \cap T)^{\nu}\right)<\infty,
$$

where the finiteness of the second product follows from Lemma 5.3.

Lemma 5.5. As the finite subset $\mathcal{T}$ tends to $\mathcal{T}_{g g^{\prime}}$, the limit

$$
\Sigma_{g g^{\prime}}=\prod_{T \in \mathcal{T}_{g g^{\prime}}} \Sigma_{T}=\lim _{\mathcal{T} \rightarrow \mathcal{T}_{g g^{\prime}}} \Sigma_{\mathcal{T}}
$$

exists in $\mathrm{SL}_{n}(\mathbb{R})$.

Proof. Let $\mathcal{T}=\left\{T_{1}, T_{2}, \ldots, T_{m}\right\}$ be a finite subset of $\mathcal{T}_{g g^{\prime}}$, where the $T_{i}$ are ordered from $g$ to $g^{\prime}$. If $\mathcal{T}^{\prime}=\mathcal{T} \cup\{T\}$ has one more element $T \in \mathcal{T}_{g g^{\prime}}$, and if $T$ separates $T_{i}$ from $T_{i+1}$, set $\mathcal{T}_{1}=\left\{T_{1}, T_{2}, \ldots, T_{i}\right\}$ and $\mathcal{T}_{2}=\left\{T_{i+1}, T_{2}, \ldots, T_{m}\right\}$; then

$$
\left\|\Sigma_{\mathcal{T}^{\prime}}-\Sigma_{\mathcal{T}}\right\|=\left\|\Sigma_{\mathcal{T}_{1}} \circ\left(\Sigma_{T}-\operatorname{Id}_{\mathbb{R}^{n}}\right) \circ \Sigma_{\mathcal{T}_{2}}\right\|=O\left(\ell(k \cap T)^{\nu}\right)
$$

by Lemmas 5.2 and 5.4. Lemma 5.3 then shows that, as $\mathcal{T}$ ranges over all finite subsets of $\mathcal{T}_{g g^{\prime}}$, the family of maps $\Sigma_{\mathcal{T}} \in \mathrm{SL}_{n}(\mathbb{R})$ satisfies the Cauchy property. The limit therefore exists.

Having defined the slithering map $\Sigma_{g g^{\prime}}: \mathbb{R}^{n} \rightarrow \mathbb{R}^{n}$, we now show that it satisfies the properties of Proposition 5.1. We begin with condition (1).

Lemma 5.6. For any two leaves $g$ and $g^{\prime}$ of $\tilde{\lambda}$, one has $\Sigma_{g g}=\operatorname{Id}_{\mathbb{R}^{n}}$ and $\Sigma_{g^{\prime} g}=\Sigma_{g g^{\prime}}^{-1}$. In addition, $\Sigma_{g g^{\prime \prime}}=\Sigma_{g g^{\prime}} \circ \Sigma_{g^{\prime} g^{\prime \prime}}$ when one of the three leaves $g, g^{\prime}$ and $g^{\prime \prime}$ separates the other two. 
In the missing case where none of $g, g^{\prime}$ and $g^{\prime \prime}$ separates the other two, the relationship between $\Sigma_{g g^{\prime \prime}}, \Sigma_{g g^{\prime}}$ and $\Sigma_{g^{\prime} g^{\prime \prime}}$ is more complicated and involves the triangle invariants of the unique component $T$ of $\widetilde{S} \backslash \tilde{\lambda}$ that separates these three leaves, as we will implicitly see in $\S 5.2$ (and in particular in the proof of Proposition 5.15).

Proof. The first two properties are immediate from definitions. When $g^{\prime}$ separates $g$ from $g^{\prime \prime}, \mathcal{T}_{g g^{\prime \prime}}$ is the disjoint union of $\mathcal{T}_{g g^{\prime}}$ and $\mathcal{T}_{g^{\prime} g^{\prime \prime}}$, and the property that $\Sigma_{g g^{\prime \prime}}=$ $\Sigma_{g g^{\prime}} \circ \Sigma_{g^{\prime} g^{\prime \prime}}$ is again an immediate consequence of the construction. The other two cases follow from this one by an algebraic manipulation.

We now turn to condition (2).

Lemma 5.7. The slithering map $\Sigma_{g g^{\prime}}$ provided by Lemma 5.5 depends Hölder continuously on the leaves $g, g^{\prime} \subset \tilde{\lambda}$ meeting the tightly transverse arc $k$.

Proof. If the leaf $h$ is close to $g$, and if the leaf $h^{\prime}$ is close to the leaf $g^{\prime}$, we can apply Lemma 5.6 to decompose $\Sigma_{h h^{\prime}}$ as

$$
\Sigma_{h h^{\prime}}=\Sigma_{h g^{\circ}} \Sigma_{g g^{\prime}} \circ \Sigma_{g^{\prime} h^{\prime}}
$$

The argument used in the proof of Lemma 5.5 shows that, for some $\nu>0$,

$$
\left\|\Sigma_{h g}-\operatorname{Id}_{\mathbb{R}^{n}}\right\|=O\left(\sum_{T \in \mathcal{T}_{h g}} \ell(k \cap T)^{\nu}\right) .
$$

By Lemma 5.3, the above series is dominated by a geometric series and, using the precise estimate provided by the second half of that statement,

$$
\begin{aligned}
\sum_{T \in \mathcal{T}_{h g}} \ell(k \cap T)^{\nu} & =O\left(\max _{T \in \mathcal{T}_{h g}} e^{-\nu C^{\prime} r(T)}\right)=O\left(\max _{T \in \mathcal{T}_{h g}} \ell(k \cap T)^{\nu^{\prime}}\right) \\
& =O\left(\ell\left(k_{h g}\right)^{\nu^{\prime}}\right)=O\left(d(h, g)^{\nu^{\prime}}\right)
\end{aligned}
$$

for $\nu^{\prime}=\nu C^{\prime} / C$ with the constants $C, C^{\prime}>0$ of Lemma 5.3, and where $k_{h g}$ is the subarc of $k$ that joins the two points $k \cap g$ and $k \cap h$. Therefore,

$$
\left\|\Sigma_{h g}-\operatorname{Id}_{\mathbb{R}^{n}}\right\|=O\left(d(g, h)^{\nu^{\prime}}\right) .
$$

Similarly,

$$
\left\|\Sigma_{g^{\prime} h^{\prime}}-\operatorname{Id}_{\mathbb{R}^{n}}\right\|=O\left(d\left(g^{\prime}, h^{\prime}\right)^{\nu^{\prime}}\right) .
$$

Combining these two estimates with the bound provided by Lemma 5.4,

$$
\begin{aligned}
\left\|\Sigma_{h h^{\prime}}-\Sigma_{g g^{\prime}}\right\| & \leqslant\left\|\Sigma_{h h^{\prime}}-\Sigma_{g h^{\prime}}\right\|+\left\|\Sigma_{g h^{\prime}}-\Sigma_{g g^{\prime}}\right\| \\
& \leqslant\left\|\Sigma_{h g}-\operatorname{Id}_{\mathbb{R}^{n}}\right\|\left\|\Sigma_{g h^{\prime}}\right\|+\left\|\Sigma_{g g^{\prime}}\right\|\left\|\Sigma_{g^{\prime} h^{\prime}}-\operatorname{Id}_{\mathbb{R}^{n}}\right\| \\
& =O\left(d(g, h)^{\nu^{\prime}}+d\left(g^{\prime}, h^{\prime}\right)^{\nu^{\prime}}\right),
\end{aligned}
$$

which proves that the map $\left(g, g^{\prime}\right) \mapsto \Sigma_{g g^{\prime}} \in \mathrm{SL}_{n}(\mathbb{R})$ is Hölder continuous over the square of the space of leaves of $\tilde{\lambda}$ that cross the arc $k$. 


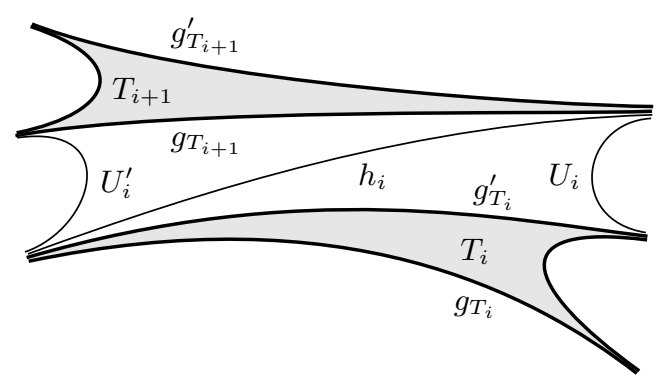

Figure 5. Adding triangles between $T_{i}$ and $T_{i+1}$.

Lemma 5.7 proves the local Hölder continuity condition (2) of Proposition 5.1.

If the leaves $g$ and $g^{\prime}$ share a common endpoint $x \in \partial_{\infty} \widetilde{S}$, then all leaves of $\tilde{\lambda}$ that separate $g$ from $g^{\prime}$ also have $x$ as an endpoint. In particular, $\Sigma_{g g^{\prime}}$ is defined as an infinite product of elementary slitherings $\Sigma_{T}=\Sigma_{g_{T} g_{T}^{\prime}}$ that respect the flag $E=\mathcal{F}_{\varrho}(x)$ and act as the identity on each line $E^{(a)} / E^{(a-1)}$. It follows that $\Sigma_{g g^{\prime}}$ satisfies the same property, which proves condition (3) of Proposition 5.1.

LEMma 5.8. Suppose that the leaves $g, g^{\prime} \subset \tilde{\lambda}$ are oriented in parallel. Then, the slithering map $\Sigma_{g g^{\prime}}$ provided by Lemma 5.5 sends the line decomposition $\mathbb{R}^{n}=\bigoplus_{a=1}^{n} \tilde{L}_{a}\left(g^{\prime}\right)$ to the line decomposition $\mathbb{R}^{n}=\bigoplus_{a=1}^{n} \tilde{L}_{a}(g)$.

Proof. The strategy is to approximate by a finite lamination the part of $\tilde{\lambda}$ that separates $g$ from $g^{\prime}$. The slithering map associated with this finite lamination will send the line decomposition $\mathbb{R}^{n}=\bigoplus_{a=1}^{n} \tilde{L}_{a}\left(g^{\prime}\right)$ to the line decomposition $\mathbb{R}^{n}=\bigoplus_{a=1}^{n} \tilde{L}_{a}(g)$, and approximate the slithering map $\Sigma_{g g^{\prime}}$. Passing to the limit in the approximation process will conclude the proof.

Let $\mathcal{T}=\left\{T_{1}, T_{2} \ldots, T_{m}\right\}$ be a finite subset of $\mathcal{T}_{g g^{\prime}}$, where the $T_{i}$ are ordered from $g$ to $g^{\prime}$. We insert two triangles $U_{i}$ and $U_{i}^{\prime}$ between $T_{i}$ and $T_{i+1}$ as follows. Recall that $g_{T_{i}}$ and $g_{T_{i}}^{\prime}$ are the two sides of $T_{i}$ separating $g$ from $g^{\prime}$, with $g_{T_{i}}$ closest to $g$. Let $h_{i}$ be the geodesic of $\widetilde{S}$ that joins the left-hand side (as seen from $g$ ) endpoint of $g_{T_{i}}^{\prime}$ to the right-hand side endpoint of $g_{T_{i+1}}$. The two geodesics $g_{T_{i}}^{\prime}$ and $h_{i}$ are two sides of a unique ideal triangle $U_{i} \subset \widetilde{S}$, possibly reduced to a single geodesic when $g_{T_{i}}^{\prime}=h_{i}$. We can similarly consider the ideal triangle $U_{i}^{\prime}$, possibly reduced to a single geodesic, with sides $h_{i}$ and $g_{T_{i+1}}$. See Figure 5 . The same construction with the conventions that $g_{T_{0}}^{\prime}=g$ and $g_{T_{m+1}}=g^{\prime}$ also defines triangles $U_{0}, U_{0}^{\prime}, U_{m}$ and $U_{m}^{\prime}$.

As before, the triangles $U_{i}$ and $U_{i}^{\prime}$ define an elementary slithering map $\Sigma_{U_{i}}$ sending the line decomposition $\mathbb{R}^{n}=\bigoplus_{a=1}^{n} \tilde{L}_{a}\left(h_{i}\right)$ to the line decomposition $\mathbb{R}^{n}=\bigoplus_{a=1}^{n} \tilde{L}_{a}\left(g_{T_{i}}^{\prime}\right)$, and another one $\Sigma_{U_{i}^{\prime}}$ sending the line decomposition $\mathbb{R}^{n}=\bigoplus_{a=1}^{n} \tilde{L}_{a}\left(g_{T_{i+1}}\right)$ to the line decomposition $\mathbb{R}^{n}=\bigoplus_{a=1}^{n} \tilde{L}_{a}\left(h_{i}\right)$. These slithering maps are equal to the identity when 
the corresponding triangles are reduced to geodesics.

Now consider

$$
\begin{gathered}
\widehat{\Sigma}_{\mathcal{T}}=\left(\Sigma_{U_{0}} \circ \Sigma_{U_{0}^{\prime}}\right) \circ \Sigma_{T_{1}} \circ\left(\Sigma_{U_{1}} \circ \Sigma_{U_{1}^{\prime}}\right) \circ \Sigma_{T_{2}} \circ\left(\Sigma_{U_{2}} \circ \Sigma_{U_{2}^{\prime}}\right) \circ \Sigma_{T_{3}} \circ \ldots \\
\ldots \circ \Sigma_{T_{m-1}} \circ\left(\Sigma_{U_{m-1}} \circ \Sigma_{U_{m-1}^{\prime}}\right) \circ \Sigma_{T_{m}} \circ\left(\Sigma_{U_{m}} \circ \Sigma_{U_{m}^{\prime}}\right) .
\end{gathered}
$$

By construction, $\widehat{\Sigma}_{\mathcal{T}}$ sends the line decomposition

$$
\mathbb{R}^{n}=\bigoplus_{a=1}^{n} \tilde{L}_{a}\left(g_{T_{m+1}}\right)=\bigoplus_{a=1}^{n} \tilde{L}_{a}\left(g^{\prime}\right)
$$

to the line decomposition

$$
\mathbb{R}^{n}=\bigoplus_{a=1}^{n} \tilde{L}_{a}\left(g_{T_{0}}^{\prime}\right)=\bigoplus_{a=1}^{n} \tilde{L}_{a}(g)
$$

To compare $\widehat{\Sigma}_{\mathcal{T}}$ and $\Sigma_{\mathcal{T}}$, choose an arc $k$ tightly transverse to $\tilde{\lambda}$ and meeting both $g$ and $g^{\prime}$. Then, Lemma 5.2 provides constants $A, \nu>0$ such that $\left\|\Sigma_{U_{i}}-\operatorname{Id}_{\mathbb{R}^{n}}\right\| \leqslant A \ell\left(k \cap U_{i}\right)^{\nu}$ and $\left\|\Sigma_{U_{i}^{\prime}}-\operatorname{Id}_{\mathbb{R}^{n}}\right\| \leqslant A \ell\left(k \cap U_{i}^{\prime}\right)^{\nu}$.

Since a $\nu$-Hölder continuous function on a compact set is also $\nu^{\prime}$-Hölder continuous for every $\nu^{\prime}<\nu$, we can assume that $\nu \leqslant 1$ without loss of generality. Then, with this condition,

$$
\ell\left(k \cap U_{i}\right)^{\nu} \leqslant \ell\left(k \cap\left(U_{i} \cup U_{i}^{\prime}\right)\right)^{\nu} \leqslant \sum_{T \in \mathcal{T}_{{ }_{{ }^{\prime}}{ }_{T_{i}}{ }^{g} T_{i+1}}} \ell(k \cap T)^{\nu},
$$

where the sum is over all components $T$ of $\widetilde{S} \backslash \tilde{\lambda}$ that separate $T_{i}$ from $T_{i+1}$. A similar estimate holds for $\ell\left(k \cap U_{i}^{\prime}\right)^{\nu}$. It follows that

$$
\left\|\Sigma_{U_{i}} \circ \Sigma_{U_{i}^{\prime}}-\operatorname{Id}_{\mathbb{R}^{n}}\right\|=O\left(\sum_{T \in \mathcal{T}_{g_{T_{i}}^{\prime} g_{T_{i+1}}}} \ell(k \cap T)^{\nu}\right) .
$$

The arguments used in the proof of Lemmas 5.4 and 5.5 can then be applied to show that

$$
\left\|\widehat{\Sigma}_{\mathcal{T}}-\Sigma_{\mathcal{T}}\right\|=O\left(\sum_{T \in \mathcal{T}_{g g^{\prime}}-\mathcal{T}} \ell(k \cap T)^{\nu}\right) .
$$

Lemma 5.3 then shows that $\widehat{\Sigma}_{\mathcal{T}}$ and $\Sigma_{\mathcal{T}}$ have the same limit as the finite subset $\mathcal{T}$ tends to $\mathcal{T}_{g g^{\prime}}$. Therefore, $\widehat{\Sigma}_{\mathcal{T}}$ also converges to the slithering map $\Sigma_{g g^{\prime}}$ as $\mathcal{T}$ tends to $\mathcal{T}_{g g^{\prime}}$.

We already observed that each $\widehat{\Sigma}_{\mathcal{T}}$ sends the line decomposition $\mathbb{R}^{n}=\bigoplus_{a=1}^{n} \tilde{L}_{a}\left(g^{\prime}\right)$ to the line decomposition $\mathbb{R}^{n}=\bigoplus_{a=1}^{n} \tilde{L}_{a}(g)$. Passing to the limit, we conclude that $\Sigma_{g g^{\prime}}$ has the same property. 
Lemma 5.8 proves condition (4) of Proposition 5.1.

We already observed in Lemma 5.5 that $\Sigma_{g g^{\prime}}$ has determinant 1 , which is condition (5).

The only property of Proposition 5.1 remaining to prove is the uniqueness of the slithering map.

LEMMA 5.9. If a family of linear isomorphisms $\Sigma_{g g^{\prime}}^{\prime}: \mathbb{R}^{n} \rightarrow \mathbb{R}^{n}$, indexed by all pairs of leaves $g, g^{\prime} \subset \tilde{\lambda}$, satisfies conditions (1)-(3) of Proposition 5.1, then $\Sigma_{g g^{\prime}}^{\prime}$ is equal to the map $\Sigma_{g g^{\prime}}$ constructed above for every $g$ and $g^{\prime}$.

In particular, conditions (4) and (5) are consequences of conditions (1)-(3).

Proof. As usual, let $k$ be a tightly transverse arc that crosses both $g$ and $g^{\prime}$. Let $\mathcal{T}=\left\{T_{1}, T_{2}, \ldots, T_{m}\right\}$ be a finite subset of the set $\mathcal{T}_{g g^{\prime}}$ of components of $\widetilde{S} \backslash \tilde{\lambda}$ that separate $g$ from $g^{\prime}$, indexed in such a way that the $T_{i}$ occur in this order as one goes from $g$ to $g^{\prime}$. Let $g_{T_{i}}$ and $g_{T_{i}}^{\prime}$ be the sides of $T_{i}$ that are closest to $g$ and $g^{\prime}$, respectively.

By condition (1),

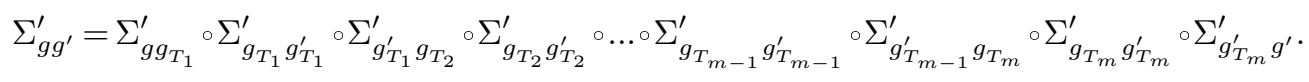

Condition (3) implies that $\Sigma_{g_{T_{i}} g_{T_{i}}^{\prime}}^{\prime}=\Sigma_{g_{T_{i}} g_{T_{i}}^{\prime}}=\Sigma_{T_{i}}$, so that

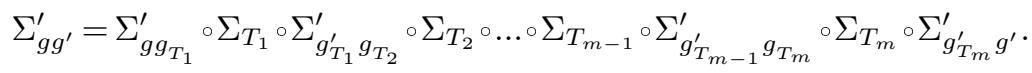

By condition (2), the map $\left(h, h^{\prime}\right) \mapsto \Sigma_{h h^{\prime}}^{\prime}$ is Hölder continuous over the space of leaves of $\tilde{\lambda}$ that meet the arc $k$. As a consequence, there exists a constant $\nu>0$ such that, for every $i$,

$$
\left\|\Sigma_{g_{T_{i}}^{\prime} g_{T_{i+1}}}^{\prime}-\operatorname{Id}_{\mathbb{R}^{n}}\right\|=O\left(d\left(g_{T_{i}}^{\prime}, g_{T_{i+1}}\right)^{\nu}\right) .
$$

Because the leaves $g_{T_{i}}^{\prime}$ and $g_{T_{i+1}}$ are disjoint, a classical estimate in negative curvature geometry (see, for instance, $[13, \S 5.2 .6])$ shows that $d\left(g_{T_{i}}^{\prime}, g_{T_{i+1}}\right)$ is bounded by a constant times the length $\ell\left(k_{g_{T_{i}}^{\prime} g_{T_{i+1}}}\right)$ of the subarc $k_{g_{T_{i}}^{\prime} g_{T_{i+1}}} \subset k$ delimited by the points $k \cap g_{T_{i}}^{\prime}$ and $k \cap g_{T_{i+1}}$.

The geodesic lamination $\tilde{\lambda}$ has area zero $([39, \S 8.5],[1])$. Its intersection with the transverse arc $k$ therefore has length zero, and

$$
\ell\left(k_{g_{T_{i}}^{\prime} g_{T_{i+1}}}\right)=\sum_{T \in \mathcal{T}_{g_{T_{i}}^{\prime}{ }^{g} T_{i+1}}} \ell(k \cap T) .
$$

Assuming $\nu \leqslant 1$ without loss of generality, we can combine all these estimates and conclude that

$$
\left\|\Sigma_{g_{T_{i}}^{\prime} g_{T_{i+1}}^{\prime}}^{\prime}-\operatorname{Id}_{\mathbb{R}^{n}}\right\|=O\left(\sum_{T \in \mathcal{T}_{g_{T_{i}}^{\prime} g_{T_{i+1}}}} \ell(k \cap T)^{\nu}\right) .
$$


This also holds for $i=0$ and $i=m$, with the convention that $g_{T_{0}}^{\prime}=g$ and $g_{T_{m+1}}=g^{\prime}$.

From this estimate, we can then use the arguments of the proofs of Lemmas 5.4 and 5.5 to show that

$$
\left\|\Sigma_{g g^{\prime}}^{\prime}-\Sigma_{\mathcal{T}}\right\|=O\left(\sum_{T \in \mathcal{T}_{g g^{\prime}}-\mathcal{T}} \ell(k \cap T)^{\nu}\right) .
$$

By Lemma 5.3, this proves that

$$
\Sigma_{g g^{\prime}}^{\prime}=\lim _{\mathcal{T} \rightarrow \mathcal{T}_{g g^{\prime}}} \Sigma_{\mathcal{T}}=\Sigma_{g g^{\prime}},
$$

which concludes the proof of Lemma 5.9.

This uniqueness property completes the proof of Proposition 5.1.

Remark 5.10. In Proposition 5.1 (and in Lemma 5.9), the uniqueness property would be false without the hypothesis that the slithering map $\Sigma_{g g^{\prime}}$ depends locally Hölder continuously (and not just continuously) on the leaves $g$ and $g^{\prime}$. This is already true in the classical case where $n=2$. Indeed, let $\alpha$ be a transverse measure for $\lambda$ that has no atom (which is automatic if $\lambda$ has no closed leaf). The atom-free hypothesis guarantees that the $\alpha$-mass $\alpha\left(g, g^{\prime}\right)$ of the set of leaves of $\tilde{\lambda}$ separating $g$ from $g^{\prime}$ depends continuously on $g$ and $g^{\prime}$. If $g$ and $g^{\prime}$ are oriented in parallel in such a way that $g^{\prime}$ is to the left of $g$, let $\Sigma_{g g^{\prime}}^{\prime}: \mathbb{R}^{2} \rightarrow \mathbb{R}^{2}$ be obtained by postcomposing the slithering map $\Sigma_{g g^{\prime}}$ with the linear map that acts by multiplication by $e^{-\alpha\left(g, g^{\prime}\right)}$ on the line $\tilde{L}_{1}(g)$, and by multiplication by $e^{\alpha\left(g, g^{\prime}\right)}$ on $\tilde{L}_{2}(g)$. This new family of maps $\Sigma_{g g^{\prime}}^{\prime}$ satisfies conditions (1) and (3)-(5) of Proposition 5.1, the maps $\Sigma_{g g^{\prime}}^{\prime}$ depend continuously (but not locally Hölder continuously) on $g$ and $g^{\prime}$, and they are of course different from the original family of slithering maps $\Sigma_{g g^{\prime}}$ if $\alpha$ is non-trivial.

To generalize this construction to all $n$, let $\alpha_{1}, \alpha_{2}, \ldots, \alpha_{n-1}$ be atom-free transverse measures for $\lambda$ such that $\alpha_{n-a}=\alpha_{a}$ for every $a$ (so that in practice we only have $\left\lfloor\frac{1}{2} n\right\rfloor$ such $\left.\alpha_{a}\right)$. For two leaves $g$ and $g^{\prime}$ of $\tilde{\lambda}$, define $\beta_{1}\left(g, g^{\prime}\right), \beta_{2}\left(g, g^{\prime}\right), \ldots, \beta_{n}\left(g, g^{\prime}\right)$ by the properties that $\alpha_{a}\left(g, g^{\prime}\right)=\beta_{a+1}\left(g, g^{\prime}\right)-\beta_{a}\left(g, g^{\prime}\right)$ and $\sum_{a=1}^{n} \beta_{a}\left(g, g^{\prime}\right)=0$. If $g$ and $g^{\prime}$ are oriented in parallel in such a way that $g^{\prime}$ is to the left of $g$, let $\Sigma_{g g^{\prime}}^{\prime}: \mathbb{R}^{n} \rightarrow \mathbb{R}^{n}$ be obtained by postcomposing the slithering map $\Sigma_{g g^{\prime}}$ with the linear map that respects each line $\tilde{L}_{a}(g)$ and acts by $e^{\beta_{a}\left(g, g^{\prime}\right)}$ on $\tilde{L}_{a}(g)$. The maps $\Sigma_{g g^{\prime}}^{\prime}$ satisfy conditions $(1)$ and $(3)-(5)$ of Proposition 5.1, and depend continuously on $g$ and $g^{\prime}$. They clearly differ from the slithering maps $\Sigma_{g g^{\prime}}$ if at least one of the $\alpha_{a}$ is non-zero. This construction automatically generalizes to the situation where the $\alpha_{a}$ are topological differential forms in the sense of [26], in which case it completely describes how the uniqueness can fail if we remove the Hölder condition from Proposition 5.1. 

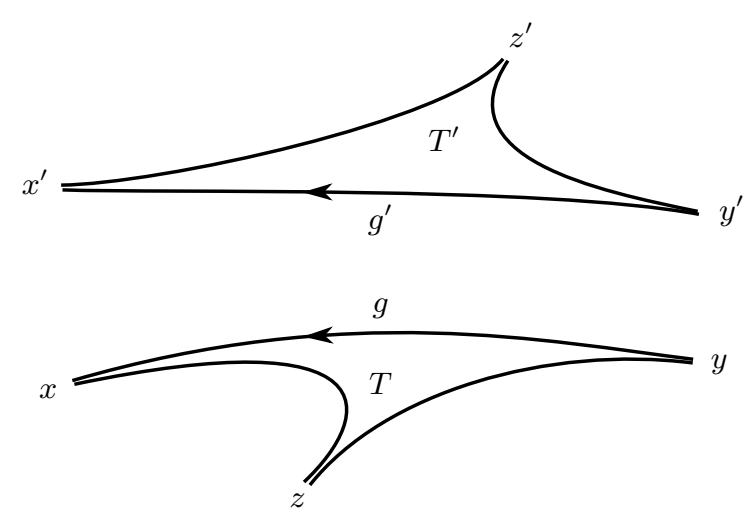

Figure 6 . The triangles $T$ and $T^{\prime}$ and their vertices.

\subsection{The shearing cycle}

We now use the slithering map to associate with the Hitchin homomorphism

$$
\varrho: \pi_{1}(S) \longrightarrow \mathrm{PSL}_{n}(\mathbb{R})
$$

a certain twisted tangent cycle $\sigma^{\varrho} \in \mathcal{Z}\left(\lambda\right.$, slits; $\left.\widehat{\mathbb{R}}^{n-1}\right)$ relative to the slits of $\lambda$. This relative tangent cycle is the shearing cycle of the Hitchin homomorphism $\varrho$.

Unlike $\S 5.1$, this construction uses in a fundamental way the positivity property of Theorem 3.3; see Lemma 5.11.

We will use the point of view of $\S 4.7$. Let $T$ and $T^{\prime}$ be two components of $\widetilde{S} \backslash \tilde{\lambda}$.

Let $g$ be the side of $T$ that is closest to $T^{\prime}$, and let $g^{\prime}$ be the side of $T^{\prime}$ closest to $T$. We orient these two leaves of $\tilde{\lambda}$ to the left as seen from $T$. In particular, $g$ and $g^{\prime}$ are oriented in parallel, and the slithering map $\Sigma_{g g^{\prime}}: \mathbb{R}^{n} \rightarrow \mathbb{R}^{n}$ of Proposition 5.1 sends each line $\tilde{L}_{a}\left(g^{\prime}\right)$ to the line $\tilde{L}_{a}(g)$.

Let $x, y \in \partial_{\infty} \widetilde{S}$ be the positive and negative endpoints of $g$, and let $z$ be the third vertex of the ideal triangle $T$. Similarly, let $x^{\prime}, y^{\prime} \in \partial_{\infty} \widetilde{S}$ be the positive and negative endpoints of $g^{\prime}$, and let $z^{\prime}$ be the third vertex of $T^{\prime}$. See Figure 6 . The flag curve $\mathcal{F}_{\varrho}: \partial_{\infty} \widetilde{S} \rightarrow \operatorname{Flag}\left(\mathbb{R}^{n}\right)$ of Proposition 3.2 now associates six flags

$$
\mathcal{F}_{\varrho}(x), \mathcal{F}_{\varrho}(y), \mathcal{F}_{\varrho}(z), \mathcal{F}_{\varrho}\left(x^{\prime}\right), \mathcal{F}_{\varrho}\left(y^{\prime}\right), \mathcal{F}_{\varrho}\left(z^{\prime}\right) \in \operatorname{Flag}\left(\mathbb{R}^{n}\right)
$$

with these vertices. By our definitions, the slithering map $\Sigma_{g g^{\prime}}$ sends $\mathcal{F}_{\varrho}\left(x^{\prime}\right)$ to $\mathcal{F}_{\varrho}(x)$ and $\mathcal{F}_{\varrho}\left(y^{\prime}\right)$ to $\mathcal{F}_{\varrho}(y)$.

We want to consider the double ratio $D_{a}\left(\mathcal{F}_{\varrho}(x), \mathcal{F}_{\varrho}(y), \mathcal{F}_{\varrho}(z), \Sigma_{g g^{\prime}}\left(\mathcal{F}_{\varrho}\left(z^{\prime}\right)\right)\right)$, as in $\S 1.4$.

Lemma 5.11. The double ratio $D_{a}\left(\mathcal{F}_{\varrho}(x), \mathcal{F}_{\varrho}(y), \mathcal{F}_{\varrho}(z), \Sigma_{g g^{\prime}}\left(\mathcal{F}_{\varrho}\left(z^{\prime}\right)\right)\right)$ is finite and positive. 
Proof. When $T$ and $T^{\prime}$ are adjacent so that $g=g^{\prime}$, then $\Sigma_{g g^{\prime}}=\operatorname{Id}_{\mathbb{R}^{n}}$ and the statement is an immediate consequence of the positivity property of Theorem 3.3. In the general case, however, the appearance of the slithering map $\Sigma_{g g^{\prime}}$ requires a more elaborate argument. The key ingredient is a deeper consequence of the positivity property, which is that the family of the line bundles $L_{a} \rightarrow T^{1} S$ of Theorem 3.1 carries a canonical joint orientation, as we now define.

A joint orientation for the line bundles $L_{a}$ is the choice of an orientation for (the fibers of) each bundle, but determined only up to simultaneous reversal of all orientations. More formally, a joint orientation for the bundles $L_{a}$ is an orientation for each of the line bundles $L_{a} \otimes L_{a+1}$.

Actually, we will see that the line bundles $L_{a}$ admit two equally canonical but opposite joint orientations: the left-hand-side and right-hand-side joint orientations.

To define these joint orientations, focus attention on a point $\tilde{u} \in T^{1} \widetilde{S}$. As in $\S 3.1$, consider the line decomposition $\mathbb{R}^{n}=\bigoplus_{a=1}^{n} \tilde{L}_{a}(\tilde{u})$ defined by the fibers over $\tilde{u}$ of the line bundles $\tilde{L}_{a} \rightarrow T^{1} \widetilde{S}$ lifting the bundles $L_{a} \rightarrow T^{1} S$. Then, if $p, q \in \partial_{\infty} \widetilde{S}$ are the positive and negative endpoints of the orbit $g$ of $\tilde{u}$ under the geodesic flow, we have

$$
\tilde{L}_{a}(\tilde{u})=\mathcal{F}_{\varrho}(p)^{(a)} \cap \mathcal{F}_{\varrho}(q)^{(n-a+1)},
$$

by definition of the flag curve $\mathcal{F}_{\varrho}$ in Proposition 3.2. Consider another point $r \in \partial_{\infty} \widetilde{S}$ that is different from $p$ and $q$, and that sits to the left of $p$ as seen from $q$. By Theorem 3.3, the flag triple $\left(\mathcal{F}_{\varrho}(p), \mathcal{F}_{\varrho}(q), \mathcal{F}_{\varrho}(r)\right)$ is generic. As a consequence, if $v$ is a non-trivial vector in the line $\mathcal{F}_{\varrho}(r)^{(1)}$, the projection of $v \in \mathbb{R}^{n}=\bigoplus_{b=1}^{n} \tilde{L}_{b}(\tilde{u})$ to the line $\tilde{L}_{a}(\tilde{u})$ parallel to all $\tilde{L}_{b}(\tilde{u})$ with $b \neq a$ is non-trivial, and therefore specifies an orientation for $\tilde{L}_{a}(\tilde{u})$. Replacing $v$ by any other non-trivial vector $v^{\prime} \in \mathcal{F}_{\varrho}(r)^{(1)}$ determines the same orientation on $\tilde{L}_{a}(\tilde{u})$ if the ratio $v / v^{\prime}$ in the line $\mathcal{F}_{\varrho}(r)^{(1)}$ is positive, or reverses all these orientations if $v / v^{\prime}<0$. Therefore, the joint orientation of the lines $\tilde{L}_{a}(\tilde{u})$ is independent of the choice of $v \in \mathcal{F}_{\varrho}(r)^{(1)}$.

To show that the joint orientation of the lines $\tilde{L}_{a}(\tilde{u})$ is independent of the choice of the point $r \in \partial_{\infty} \widetilde{S}$, consider another point $r^{\prime} \in \partial_{\infty} \widetilde{S}$ different from $p$ and $q$, and now located on the right of $p$ as seen from $q$. This point $r^{\prime}$ similarly defines a joint orientation for the lines $\tilde{L}_{a}(\tilde{u})$, and we will see that this joint orientation is exactly the opposite of that defined by $r$ (considering joint orientations as orientations of the line bundles $\left.L_{a} \otimes L_{a+1}\right)$. To prove this, pick non-trivial vectors $v \in \mathcal{F}_{\varrho}(r)^{(1)}$ and $v^{\prime} \in \mathcal{F}_{\varrho}\left(r^{\prime}\right)^{(1)}$. Let $v_{a}$ and $v_{a}^{\prime}$ denote the respective projections of $v$ and $v^{\prime}$ to the line $\tilde{L}_{a}(\tilde{u})$ parallel to all $\tilde{L}_{b}(\tilde{u})$ with $b \neq a$. Since $r$ and $r^{\prime}$ are in different components of $\partial_{\infty} \widetilde{S} \backslash\{p, q\}$, Lemma 1.6 and the positivity condition of Theorem 3.3 show that

$$
0<D_{a}\left(\mathcal{F}_{\varrho}(p), \mathcal{F}_{\varrho}(q), \mathcal{F}_{\varrho}(r), \mathcal{F}_{\varrho}\left(r^{\prime}\right)\right)=-\frac{v_{a+1}}{v_{a+1}^{\prime}} \frac{v_{a}^{\prime}}{v_{a}},
$$


where the ratios $v_{b}^{\prime} / v_{b} \in \mathbb{R} \backslash\{0\}$ are computed in the lines $\tilde{L}_{b}(\tilde{u})$. As a consequence, $v$ and $v^{\prime}$ induce opposite orientations on the lines $\tilde{L}_{a}(\tilde{u}) \otimes \tilde{L}_{a+1}(\tilde{u})$. In other words, the joint orientation of the lines $\tilde{L}_{a}(\tilde{u})$ defined by the point $r^{\prime} \in \partial_{\infty} \widetilde{S}$ is the opposite of that defined by $r$. It immediately follows that the joint orientation defined by $r$ is independent of the choice of $r$ in the left-hand-side component of $\partial_{\infty} \widetilde{S} \backslash\{p, q\}$ (as seen from $q$ ).

We will refer to the joint orientation defined by $r$ as the left-hand-side joint orientation of the lines $\tilde{L}_{a}(\tilde{u})$, whereas the right-hand-side joint orientation will be the one defined by $r^{\prime}$. These two joint orientations are opposite of each other.

Let $h$ and $h^{\prime}$ be two oriented geodesics of $\widetilde{S}$ that share the same positive endpoint $p \in$ $\partial_{\infty} \widetilde{S}$, and let $\Sigma_{h h^{\prime}}: \mathbb{R}^{n} \rightarrow \mathbb{R}^{n}$ be the elementary slithering map, sending each line $\tilde{L}_{a}\left(h^{\prime}\right)$ to $\tilde{L}_{a}(h)$, defined as in Proposition $5.1(4)$. The definition of $\Sigma_{h h^{\prime}}$ through the isomorphisms

$$
\tilde{L}_{a}\left(h^{\prime}\right) \cong \mathcal{F}^{\varrho}(p)^{(a)} / \mathcal{F}^{\varrho}(p)^{(a-1)} \cong \tilde{L}_{a}(h)
$$

makes it clear that $\Sigma_{h h^{\prime}}$ sends the left-hand-side joint orientation of the family of lines $\tilde{L}_{a}\left(h^{\prime}\right)$ to the left-hand-side joint orientation of the $\tilde{L}_{a}(h)$.

We now return to the leaves $g$ and $g^{\prime}$ of $\tilde{\lambda}$. As in the proof of Lemma 5.8 and with the notation used there, approximate the part of $\tilde{\lambda}$ that separates $g$ and $g^{\prime}$ by a finite lamination, and the slithering map $\Sigma_{g g^{\prime}}$ by a finite composition

$$
\begin{gathered}
\widehat{\Sigma}_{\mathcal{T}}=\left(\Sigma_{U_{0}} \circ \Sigma_{U_{0}^{\prime}}\right) \circ \Sigma_{T_{1}} \circ\left(\Sigma_{U_{1}} \circ \Sigma_{U_{1}^{\prime}}\right) \circ \Sigma_{T_{2}} \circ\left(\Sigma_{U_{2}} \circ \Sigma_{U_{2}^{\prime}}\right) \circ \Sigma_{T_{3}} \circ \ldots \\
\ldots \circ \Sigma_{T_{m-1}} \circ\left(\Sigma_{U_{m-1}} \circ \Sigma_{U_{m-1}^{\prime}}\right) \circ \Sigma_{T_{m}} \circ\left(\Sigma_{U_{m}} \circ \Sigma_{U_{m}^{\prime}}\right)
\end{gathered}
$$

of elementary slitherings where, for any two consecutive terms, the corresponding triangles $T_{i}$ and $U_{i}$, or $U_{i}$ and $U_{i}^{\prime}$, or $U_{i}^{\prime}$ and $T_{i+1}$, share a side $g_{T_{i}}, h_{i}$ or $g_{T_{i+1}}^{\prime}$, respectively. By our earlier observation, each of these elementary slitherings respects joint orientations of the appropriate families of lines. It follows that $\widehat{\Sigma}_{\mathcal{T}}$ sends the left-hand-side joint orientation of the lines $\tilde{L}_{a}\left(g^{\prime}\right)$ to the left-hand-side joint orientation of the $\tilde{L}_{a}(g)$. Passing to the limit as the approximation $\widehat{\Sigma}_{\mathcal{T}}$ tends to $\Sigma_{g g^{\prime}}$, we conclude that the slithering map $\Sigma_{g g^{\prime}}$ sends the left-hand-side joint orientation of the lines $\tilde{L}_{a}\left(g^{\prime}\right)$ to the left-hand-side joint orientation of the $\tilde{L}_{a}(g)$.

We are now ready to determine the sign of the double ratio

$$
D_{a}\left(\mathcal{F}_{\varrho}(x), \mathcal{F}_{\varrho}(y), \mathcal{F}_{\varrho}(z), \Sigma_{g g^{\prime}}\left(\mathcal{F}_{\varrho}\left(z^{\prime}\right)\right)\right) .
$$

Pick non-trivial vectors $v$ and $v^{\prime}$ in the lines $\mathcal{F}_{\varrho}(z)^{(1)}$ and $\mathcal{F}_{\varrho}\left(z^{\prime}\right)^{(1)}$, respectively. The left-hand-side joint orientation of the family of lines $\tilde{L}_{a}(g)$ is defined by the projections $v_{a}$ of $v$ to $\tilde{L}_{a}(g)$ parallel to the other lines $\tilde{L}_{b}(g)$ with $b \neq a$. Similarly, the right-hand-side joint orientation of the lines $\tilde{L}_{a}\left(g^{\prime}\right)$ is defined by the projections $v_{a}^{\prime}$ of $v^{\prime}$ to $\tilde{L}_{a}\left(g^{\prime}\right)$ parallel 
to the lines $\tilde{L}_{b}\left(g^{\prime}\right)$ with $b \neq a$. Since we just proved that the slithering map $\Sigma_{g g^{\prime}}$ respects joint orientations, and since the left- and right-hand-side orientations are opposite of each other, the joint orientation of the $\tilde{L}_{a}(g)$ by the vectors $v_{a}$ is opposite to that defined by the vectors $\Sigma_{g g^{\prime}}\left(v_{a}^{\prime}\right)$. In other words, all ratios

$$
\frac{\Sigma_{g g^{\prime}}\left(v_{a}^{\prime}\right)}{v_{a}} \frac{v_{a+1}}{\Sigma_{g g^{\prime}}\left(v_{a+1}^{\prime}\right)}
$$

are negative. By Lemma 1.6,

$$
D_{a}\left(\mathcal{F}_{\varrho}(x), \mathcal{F}_{\varrho}(y), \mathcal{F}_{\varrho}(z), \Sigma_{g g^{\prime}}\left(\mathcal{F}_{\varrho}\left(z^{\prime}\right)\right)\right)=-\frac{v_{a+1}}{\Sigma_{g g^{\prime}}\left(v_{a+1}^{\prime}\right)} \frac{\Sigma_{g g^{\prime}}\left(v_{a}^{\prime}\right)}{v_{a}}>0,
$$

which concludes the proof of Lemma 5.11.

Lemma 5.11 enables us to define the a-th shearing parameter of the Hitchin homomorphism $\varrho$ between the components $T$ and $T^{\prime}$ of $\widetilde{S} \backslash \tilde{\lambda}$ as

$$
\sigma_{a}^{\varrho}\left(T, T^{\prime}\right)=\log D_{a}\left(\mathcal{F}_{\varrho}(x), \mathcal{F}_{\varrho}(y), \mathcal{F}_{\varrho}(z), \Sigma_{g g^{\prime}}\left(\mathcal{F}_{\varrho}\left(z^{\prime}\right)\right)\right) \in \mathbb{R} .
$$

These shearing parameters are then combined in the shearing vector

$$
\sigma^{\varrho}\left(T, T^{\prime}\right)=\left(\sigma_{1}^{\varrho}\left(T, T^{\prime}\right), \sigma_{2}\left(T, T^{\prime}\right), \ldots, \sigma_{n-1}^{\varrho}\left(T, T^{\prime}\right)\right) \in \mathbb{R}^{n-1} .
$$

We now show that the family of shearing vectors $\sigma^{\varrho}\left(T, T^{\prime}\right)$ defines a relative tangent cycle $\sigma^{\varrho} \in \mathcal{Z}\left(\lambda\right.$, slits; $\left.\widehat{\mathbb{R}}^{n-1}\right)$ for $\lambda$ valued in the twisted coefficient bundle $\widehat{\mathbb{R}}^{n}$, as in Proposition 4.9. We begin with the easier part, namely condition (3) of that statement.

Lemma 5.12. For any two components $T$ and $T^{\prime}$ of $\widetilde{S} \backslash \tilde{\lambda}$,

$$
\sigma_{a}^{\varrho}\left(T^{\prime}, T\right)=\sigma_{n-a}^{\varrho}\left(T, T^{\prime}\right) .
$$

Proof. Using the notation of Figure 6,

$$
\begin{aligned}
\sigma_{a}^{\varrho}\left(T^{\prime}, T\right) & =\log D_{a}\left(\mathcal{F}_{\varrho}\left(y^{\prime}\right), \mathcal{F}_{\varrho}\left(x^{\prime}\right), \mathcal{F}_{\varrho}\left(z^{\prime}\right), \Sigma_{g^{\prime} g}\left(\mathcal{F}_{\varrho}(z)\right)\right) \\
& =\log D_{n-a}\left(\mathcal{F}_{\varrho}\left(x^{\prime}\right), \mathcal{F}_{\varrho}\left(y^{\prime}\right), \Sigma_{g^{\prime} g}\left(\mathcal{F}_{\varrho}(z)\right), \mathcal{F}_{\varrho}\left(z^{\prime}\right)\right) \\
& =\log D_{n-a}\left(\Sigma_{g^{\prime} g}\left(\mathcal{F}_{\varrho}(x)\right), \Sigma_{g^{\prime} g}\left(\mathcal{F}_{\varrho}(y)\right), \Sigma_{g^{\prime} g}\left(\mathcal{F}_{\varrho}(z)\right), \mathcal{F}_{\varrho}\left(z^{\prime}\right)\right) \\
& =\log D_{n-a}\left(\mathcal{F}_{\varrho}(x), \mathcal{F}_{\varrho}(y), \mathcal{F}_{\varrho}(z), \Sigma_{g g^{\prime}}\left(\mathcal{F}_{\varrho}\left(z^{\prime}\right)\right)\right) \\
& =\sigma_{n-a}^{\varrho}\left(T, T^{\prime}\right),
\end{aligned}
$$

where the second equality is a consequence of the elementary properties of double ratios stated in Lemma 1.7, the third equality comes from the fact that $\Sigma_{g^{\prime} g}$ sends each line $\tilde{L}_{b}(g)$ to $\tilde{L}_{b}\left(g^{\prime}\right)$, and the fourth equality follows from the invariance of double ratios under the action of $\Sigma_{g g^{\prime}}=\Sigma_{g^{\prime} g}^{-1} \in \mathrm{SL}_{n}(\mathbb{R})$. 
The quasi-additivity property, namely condition (2) of Proposition 4.9, will involve the quadruple ratios $Q_{a}(E, F, G)$ of $\S 1.3$. The following computation, which connects quadruple ratios to double ratios and slithering maps, is the key to this relationship.

Lemma 5.13. Let $x, y, y^{\prime} \in \partial_{\infty} \widetilde{S}$ be the vertices of a component $T$ of $\widetilde{S} \backslash \tilde{\lambda}$, occurring in this order counterclockwise around $T$. Let $g$ be the side of $T$ going from $x$ to $y$, and let $g^{\prime}$ be the side going from $x$ to $y^{\prime}$. In particular, the elementary slithering map $\Sigma_{g g^{\prime}}: \mathbb{R}^{n} \rightarrow \mathbb{R}^{n}$ respects the flag $E=\mathcal{F}_{\varrho}(x) \in \operatorname{Flag}\left(\mathbb{R}^{n}\right)$ and sends $F^{\prime}=\mathcal{F}_{\varrho}\left(y^{\prime}\right)$ to $F=\mathcal{F}_{\varrho}(y)$. Then,

$$
Q_{a}\left(E, F, F^{\prime}\right)=D_{a}\left(E, F, F^{\prime}, \Sigma_{g g^{\prime}}(F)\right)^{-1} .
$$

Proof. By definition of the double ratio,

$$
D_{a}\left(E, F, F^{\prime}, \Sigma_{g g^{\prime}}(F)\right)=-\frac{e^{(a)} \wedge f^{(n-a-1)} \wedge f^{\prime(1)}}{e^{(a)} \wedge f^{(n-a-1)} \wedge \Sigma_{g g^{\prime}}\left(f^{(1)}\right)} \frac{e^{(a-1)} \wedge f^{(n-a)} \wedge \Sigma_{g g^{\prime}}\left(f^{(1)}\right)}{e^{(a-1)} \wedge f^{(n-a)} \wedge f^{\prime(1)}}
$$

for arbitrary non-zero $e^{(b)} \in \Lambda^{b}\left(E^{(b)}\right), f^{(b)} \in \Lambda^{b}\left(F^{(b)}\right), f^{\prime(b)} \in \Lambda^{b}\left(F^{\prime(b)}\right)$.

The elementary slithering map $\Sigma_{g g^{\prime}}$ respects the flag $E$ and sends $F^{\prime}$ to $F$. By condition (3) of Proposition 5.1, it acts trivially on each $\Lambda^{b}\left(E^{(b)}\right)$, including $\Lambda^{n}\left(E^{(n)}\right)=$ $\Lambda^{n}\left(\mathbb{R}^{n}\right)$. If we choose $f^{\prime(b)}=\Sigma_{g g^{\prime}}^{-1}\left(f^{(b)}\right)$, we consequently have that

$$
\begin{aligned}
e^{(b)} \wedge f^{(n-b-1)} \wedge \Sigma_{g g^{\prime}}\left(f^{(1)}\right) & =\Sigma_{g g^{\prime}}\left(e^{(b)}\right) \wedge \Sigma_{g g^{\prime}}\left(f^{\prime(n-b-1)}\right) \wedge \Sigma_{g g^{\prime}}\left(f^{(1)}\right) \\
& =\Sigma_{g g^{\prime}}\left(e^{(b)} \wedge f^{\prime(n-b-1)} \wedge f^{(1)}\right)=e^{(b)} \wedge f^{\prime(n-b-1)} \wedge f^{(1)}
\end{aligned}
$$

for every $b$. Similarly, $e^{(b)} \wedge f^{(n-b)}=e^{(b)} \wedge f^{\prime(n-b)}$ for every $b$.

Combining these properties and rearranging terms provides

$$
\begin{aligned}
D_{a} & \left(E, F, F^{\prime}, \Sigma_{g g^{\prime}}(F)\right) \\
& =-\frac{e^{(a)} \wedge f^{(n-a-1)} \wedge f^{\prime(1)}}{e^{(a)} \wedge f^{\prime(n-a-1)} \wedge f^{(1)}} \frac{e^{(a-1)} \wedge f^{\prime(n-a)} \wedge f^{(1)}}{e^{(a-1)} \wedge f^{(n-a)} \wedge f^{\prime(1)}} \\
& =\frac{e^{(a)} \wedge f^{(n-a-1)} \wedge f^{\prime(1)}}{e^{(a-1)} \wedge f^{(n-a)} \wedge f^{\prime(1)}} \frac{e^{(a-1)} \wedge f^{(1)} \wedge f^{\prime(n-a)}}{e^{(a)} \wedge f^{(1)} \wedge f^{\prime(n-a-1)}} \frac{e^{(a+1)} \wedge f^{\prime(n-a-1)}}{e^{(a+1)} \wedge f^{(n-a-1)}} \frac{e^{(a)} \wedge f^{(n-a)}}{e^{(a)} \wedge f^{\prime(n-a)}} \\
& =Q_{a}\left(E, H, H^{\prime}\right)^{-1} .
\end{aligned}
$$

Let $s$ be a slit of $\lambda$ or, equivalently, a spike of the complement $S \backslash \lambda$. Lift $s$ to a spike of $\widetilde{S} \backslash \tilde{\lambda}$, namely to a vertex $x \in \partial_{\infty} \widetilde{S}$ of a triangle component $T$ of $\widetilde{S} \backslash \tilde{\lambda}$. Let $y$ and $z$ be the other two vertices of $T$, indexed so that $x, y$ and $z$ occur in this order counterclockwise around $T$. The flag curve $\mathcal{F}_{\varrho}$ then determines a positive triple of flags $\mathcal{F}_{\varrho}(x), \mathcal{F}_{\varrho}(y)$ and $\mathcal{F}_{\varrho}(z) \in \operatorname{Flag}\left(\mathbb{R}^{n}\right)$. Considering their quadruple ratios as in $\S 1.3$, define

$$
\xi_{a}^{\varrho}(s)=\log Q_{a}\left(\mathcal{F}_{\varrho}(x), \mathcal{F}_{\varrho}(y), \mathcal{F}_{\varrho}(z)\right),
$$


which is clearly independent of the lift of the slit $s$ to the universal cover $\widetilde{S}$.

This invariant $\xi_{a}^{\varrho}(s)$ can be expressed in terms of the triangle invariants $\tau_{a b c}^{\varrho}(s)$ of $\varrho$ that we introduced in $\S 3.2$.

LemMa 5.14 .

$$
\xi_{a}^{\varrho}(s)=\sum_{b+c=n-a} \tau_{a b c}^{\varrho}(s)
$$

Proof. This is an immediate consequence of Lemma 1.5.

Recall that, by definition, a slit $\hat{s}$ of the orientation cover $\hat{\lambda}$ is positive if the canonical orientation of $\hat{\lambda}$ orients towards $\hat{s}$ the two leaves that are adjacent to $\hat{s}$, and that $\hat{s}$ is negative when these two leaves are oriented away from $\hat{s}$.

Proposition 5.15. The rule $\left(T, T^{\prime}\right) \mapsto \sigma_{a}^{\varrho}\left(T, T^{\prime}\right)$ defines a relative tangent cycle $\sigma_{a}^{\varrho} \in$ $Z(\hat{\lambda}$, slits $; \mathbb{R})$. The boundary $\partial \sigma_{a}^{\varrho}:\{$ slits of $\hat{\lambda}\} \rightarrow \mathbb{R}$ is defined by the property that, for every slit $\hat{s}$ of $\hat{\lambda}$ projecting to a slit $s$ of $\lambda$,

$$
\partial \sigma_{a}^{\varrho}(\hat{s})= \begin{cases}\xi_{a}^{\varrho}(s), & \text { if } \hat{s} \text { is a positive slit of } \hat{\lambda} \\ -\xi_{n-a}^{\varrho}(s), & \text { if } \hat{s} \text { is negative. }\end{cases}
$$

In the classical case where $n=2$, the boundary $\partial \sigma_{1}^{\varrho}$ is trivial, since there are no triangle invariants, and $\sigma_{1}^{\varrho} \in \mathcal{Z}(\hat{\lambda} ; \mathbb{R})$ is consequently a (non-relative) tangent cycle.

Proof. Using the framework of Proposition 4.8, let $T, T^{\prime}$ and $T^{\prime \prime}$ be three components of $\widetilde{S} \backslash \tilde{\lambda}$ such that $T^{\prime \prime}$ separates $T$ from $T^{\prime}$ in $\widetilde{S}$. Let $\tilde{s}^{\prime \prime}$ be the spike of $T^{\prime \prime}$ delimited by the two sides of $T^{\prime \prime}$ that separate $T$ from $T^{\prime}$.

We first consider the case where $\tilde{s}^{\prime \prime}$ points to the left as seen from $T$.

Let $g$ be the side of $T$ that is closest to $T^{\prime}$ and $T^{\prime \prime}$, and let $g^{\prime}$ be the side of $T^{\prime}$ that is closest to $T$ and $T^{\prime \prime}$. Let $f$ be the side of $T^{\prime \prime}$ that faces $T$, and let $f^{\prime}$ be the side of $T^{\prime \prime}$ that faces $T^{\prime}$. Orient these leaves of $\tilde{\lambda}$ to the left as seen from $T$. Let $E, F, E^{\prime}, F^{\prime}, E^{\prime \prime}, H, H^{\prime} \in$ $\operatorname{Flag}\left(\mathbb{R}^{n}\right)$ be the flags respectively assigned by the flag curve $\mathcal{F}_{\varrho}: \partial_{\infty} \widetilde{S} \rightarrow \mathrm{Flag}\left(\mathbb{R}^{n}\right)$ to the positive endpoint of $g$, the negative endpoint of $g$, the positive endpoint of $g^{\prime}$, the negative endpoint of $g^{\prime}$, the positive endpoint $\tilde{s}^{\prime \prime}$ of $f$ and $f^{\prime}$, the negative endpoint of $f$, and the negative endpoint of $f^{\prime}$. Similarly, let $G, G^{\prime} \in \mathrm{Flag}\left(\mathbb{R}^{n}\right)$ be respectively associated with the vertex of $T$ that is not contained in $g$, and with the vertex of $T^{\prime}$ that is not contained in $g^{\prime}$. See Figure 7, where the vertices of $T, T^{\prime}$ and $T^{\prime \prime}$ are labelled by the flags assigned to them by the flag curve $\mathcal{F}_{\varrho}$.

Then,

$$
\sigma_{a}^{\varrho}\left(T, T^{\prime}\right)=\log D_{a}\left(E, F, G, \Sigma_{g g^{\prime}}\left(G^{\prime}\right)\right)=\log D_{a}\left(E^{\prime \prime}, H, \Sigma_{f g}(G), \Sigma_{f g^{\prime}}\left(G^{\prime}\right)\right),
$$




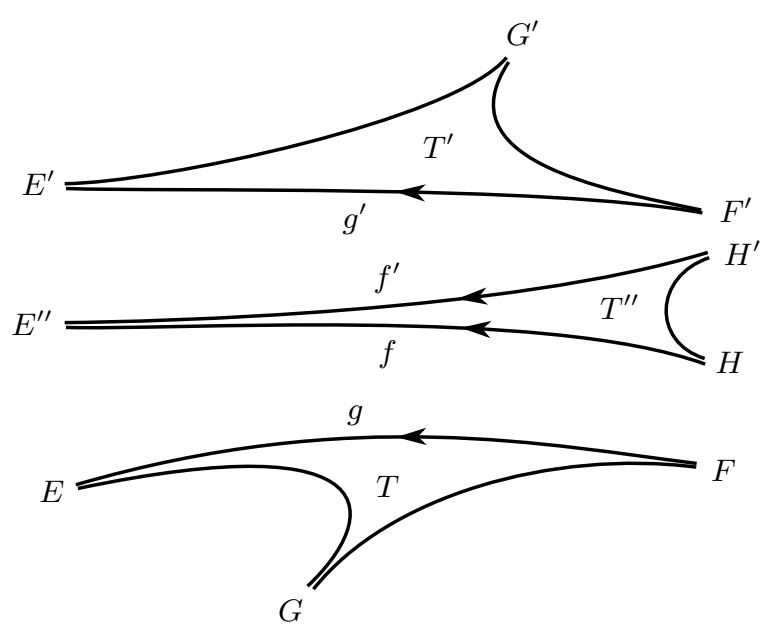

Figure 7. The triangles $T, T^{\prime}$ and $T^{\prime \prime}$, their sides, and the flags associated with their vertices.

by using the fact that the slithering map $\Sigma_{f g}$ sends $E$ to $E^{\prime \prime}$ and $F$ to $H$. Similarly,

$$
\sigma_{a}^{\varrho}\left(T, T^{\prime \prime}\right)=\log D_{a}\left(E, F, G, \Sigma_{g f}\left(H^{\prime}\right)\right)=\log D_{a}\left(E^{\prime \prime}, H, \Sigma_{f g}(G), H^{\prime}\right)
$$

and

$$
\sigma_{a}^{\varrho}\left(T^{\prime \prime}, T^{\prime}\right)=\log D_{a}\left(E^{\prime \prime}, H^{\prime}, H, \Sigma_{f^{\prime} g^{\prime}}\left(G^{\prime}\right)\right)=\log D_{a}\left(E^{\prime \prime}, H, \Sigma_{f f^{\prime}}(H), \Sigma_{f g^{\prime}}\left(G^{\prime}\right)\right)
$$

Using the elementary properties of double ratios stated in Lemma 1.7, it follows that

$$
\sigma_{a}^{\varrho}\left(T, T^{\prime}\right)=\sigma_{a}^{\varrho}\left(T, T^{\prime \prime}\right)+\sigma_{a}^{\varrho}\left(T^{\prime \prime}, T^{\prime}\right)+\log D_{a}\left(E^{\prime \prime}, H, H^{\prime}, \Sigma_{f f^{\prime}}(H)\right) .
$$

Lemma 5.13 now shows that

$$
\sigma_{a}^{\varrho}\left(T, T^{\prime}\right)=\sigma_{a}^{\varrho}\left(T, T^{\prime \prime}\right)+\sigma_{a}^{\varrho}\left(T^{\prime \prime}, T^{\prime}\right)-\log Q_{a}\left(E^{\prime \prime}, H, H^{\prime}\right)=\sigma_{a}^{\varrho}\left(T, T^{\prime \prime}\right)+\sigma_{a}^{\varrho}\left(T^{\prime \prime}, T^{\prime}\right)-\xi_{a}^{\varrho}\left(s^{\prime \prime}\right)
$$

where $s^{\prime \prime}$ is the slit of $\lambda$ that is the projection of the slit $\tilde{s}^{\prime \prime}$ of $\tilde{\lambda}$.

This computation holds when $\tilde{s}^{\prime \prime}$ points to the left as seen from $T$. When $\tilde{s}^{\prime \prime}$ points to the right, a very similar computation or an application of Lemma 5.12 shows that in this case

$$
\sigma_{a}^{\varrho}\left(T, T^{\prime}\right)=\sigma_{a}^{\varrho}\left(T, T^{\prime \prime}\right)+\sigma_{a}^{\varrho}\left(T^{\prime \prime}, T^{\prime}\right)-\xi_{n-a}^{\varrho}\left(s^{\prime \prime}\right)
$$

Considering these two cases, Proposition 4.8 then shows that the rule

$$
\left(T, T^{\prime}\right) \longmapsto \sigma_{a}^{\varrho}\left(T, T^{\prime}\right)
$$

defines a relative tangent cycle $\sigma_{a}^{\varrho} \in \mathcal{Z}(\hat{\lambda}$, slits; $\mathbb{R})$, whose boundary $\partial \sigma_{a}^{\varrho}:\{$ slits of $\hat{\lambda}\} \rightarrow \mathbb{R}$ is the one described in the statement of Proposition 5.15. This concludes the proof of that proposition. 
Through Proposition 4.9, the combination of Lemma 5.12 and Proposition 5.15 shows that the relative tangent cycles $\sigma_{a}^{\varrho} \in \mathcal{Z}(\hat{\lambda}$, slits; $\mathbb{R})$ can be combined to define a relative tangent cycle $\sigma^{\varrho} \in \mathcal{Z}\left(\lambda\right.$, slits; $\left.\widehat{\mathbb{R}}^{n-1}\right)$ valued in the twisted coefficient bundle $\widehat{\mathbb{R}}^{n-1}$ introduced in $\S 4.6$. This twisted relative tangent cycle is the shearing cycle of the Hitchin character $\varrho \in \operatorname{Hit}_{n}(S)$ with respect to the maximal geodesic lamination $\lambda$.

\section{Hitchin characters are determined by their invariants}

The goal of this section is to show that, if two Hitchin homomorphisms $\varrho, \varrho^{\prime}: \pi_{1}(S) \rightarrow$ $\mathrm{PSL}_{n}(\mathbb{R})$ have the same triangle invariants and the same shearing cycle, then they represent the same character in the Hitchin component $\operatorname{Hit}_{n}(S)$.

\subsection{Revisiting the slithering map}

We give in Proposition 6.2 below a different description of the slithering map $\Sigma_{g g^{\prime}}$ of §5.1. This new formulation is based on the following simple algebraic trick.

Lemma 6.1. Let $A_{1}, A_{2}, \ldots, A_{m}$ be elements of a group. Then,

$$
A_{1} A_{2} \ldots A_{m-1} A_{m}=\hat{A}_{m} \hat{A}_{m-1} \ldots \hat{A}_{2} \hat{A}_{1}
$$

where $\hat{A}_{i}=\left(A_{1} A_{2} \ldots A_{i-1}\right) A_{i}\left(A_{1} A_{2} \ldots A_{i-1}\right)^{-1}$.

Proof. Observe that $A_{1} A_{2} \ldots A_{m-1} A_{m}=\hat{A}_{m} A_{1} A_{2} \ldots A_{m-1}$, and proceed by induction.

We return to the construction of the slithering map $\Sigma_{g g^{\prime}}$ in $\S 5.1$. Let $g$ and $g^{\prime}$ be two leaves of the preimage $\tilde{\lambda} \subset \widetilde{S}$ of the geodesic lamination $\lambda$, and let $\mathcal{T}_{g g^{\prime}}$ be the set of components of $\widetilde{S} \backslash \tilde{\lambda}$ that separate $g$ from $g^{\prime}$, where these components are ordered from $g$ to $g^{\prime}$. For such a component $T \in \mathcal{T}_{g g^{\prime}}$, we consider the elementary slithering $\Sigma_{T}=\Sigma_{g_{T} g_{T}^{\prime}}$ defined by condition (3) of Proposition 5.1, where $g_{T}$ and $g_{T}^{\prime}$ are the two sides of $T$ that are respectively closest to $g$ and $g^{\prime}$.

We now consider the infinite product of the maps

$$
\widehat{\Sigma}_{T}=\Sigma_{g g_{T}} \circ \Sigma_{T} \circ \Sigma_{g g_{T}}^{-1} .
$$

More precisely, let $\mathcal{T}=\left\{T_{1}, T_{2}, \ldots, T_{m}\right\}$ be a finite subset of $\mathcal{T}_{g g^{\prime}}$, where each $T_{i}$ separates $T_{i+1}$ from $g$. We then consider the limit

$$
\prod_{T \in \mathcal{T}_{g g^{\prime}}} \widehat{\Sigma}_{T}=\lim _{\mathcal{T} \rightarrow \mathcal{T}_{g g^{\prime}}} \widehat{\Sigma}_{T_{m}} \circ \widehat{\Sigma}_{T_{m-1}} \circ \ldots \circ \widehat{\Sigma}_{T_{2}} \circ \widehat{\Sigma}_{T_{1}}
$$


The reverse arrow on top of the product sign is here to remind us that the composition of the $\widehat{\Sigma}_{T}$ is taken in the order opposite to the ordering of the elements of $\mathcal{T}_{g g^{\prime}}$ from $g$ to $g^{\prime}$,

Proposition 6.2. The slithering map $\Sigma_{g g^{\prime}}$ is equal to

$$
\Sigma_{g g^{\prime}}=\coprod_{T \in \mathcal{T}_{g g^{\prime}}} \widehat{\Sigma}_{T}
$$

Proof. First of all, the fact that the infinite product converges is proved by the estimates of $\S 5.1$, using the fact that the $\Sigma_{g_{g_{T}}}$ are uniformly bounded (Lemma 5.4) and the estimates on $\Sigma_{T}-\operatorname{Id}_{\mathbb{R}^{n}}$ given by Lemmas 5.2 and 5.3 .

As usual, let $\mathcal{T}=\left\{T_{1}, T_{2}, \ldots, T_{m}\right\}$ be a finite subset of $\mathcal{T}_{g g^{\prime}}$, where each $T_{i}$ separates $T_{i+1}$ from $g$. By Lemma 6.1,

$$
\prod_{T \in \mathcal{T}} \Sigma_{T}=\Sigma_{T_{1}} \circ \Sigma_{T_{2}} \circ \ldots \circ \Sigma_{T_{m-1}} \circ \Sigma_{T_{m}}=\widehat{\Sigma}_{T_{m}}^{\mathcal{T}} \circ \widehat{\Sigma}_{T_{m-1}}^{\mathcal{T}} \circ \ldots \circ \widehat{\Sigma}_{T_{2}}^{\mathcal{T}} \circ \widehat{\Sigma}_{T_{1}}^{\mathcal{T}}=\overleftarrow{\prod_{T \in \mathcal{T}}} \widehat{\Sigma}_{T}^{\mathcal{T}}
$$

where

$$
\widehat{\Sigma}_{T_{i}}^{\mathcal{T}}=\left(\Sigma_{T_{1}} \circ \Sigma_{T_{2}} \circ \ldots \circ \Sigma_{T_{i-1}}\right) \circ \Sigma_{T_{i}} \circ\left(\Sigma_{T_{1}} \circ \Sigma_{T_{2}} \circ \ldots \circ \Sigma_{T_{i-1}}\right)^{-1} .
$$

For a fixed $T$, the map $\widehat{\Sigma}_{T}^{\mathcal{T}}$ tends to $\widehat{\Sigma}_{T}=\Sigma_{g g_{T}} \circ \Sigma_{T} \circ \Sigma_{g g_{T}}^{-1}$ as the finite family $\mathcal{T}$ tends to the set $\mathcal{T}_{g g^{\prime}}$ of all components of $\widetilde{S} \backslash \tilde{\lambda}$ separating $g$ from $g^{\prime}$, by definition of the slithering map. By uniformity in the exponential estimates guaranteeing the convergence of the infinite products, it follows that

$$
\Sigma_{g g^{\prime}}=\lim _{\mathcal{T} \rightarrow \mathcal{T}_{g g^{\prime}}} \overrightarrow{\prod_{T \in \mathcal{T}}} \Sigma_{T}=\lim _{\mathcal{T} \rightarrow \mathcal{T}_{g g^{\prime}}} \overleftarrow{\prod_{T \in \mathcal{T}}} \widehat{\Sigma}_{T}^{\mathcal{T}}=\lim _{\mathcal{T} \rightarrow \mathcal{T}_{g g^{\prime}}} \overleftarrow{\prod_{T \in \mathcal{T}}} \widehat{\Sigma}_{T}=\prod_{T \in \mathcal{T}_{g g^{\prime}}} \widehat{\Sigma}_{T}
$$

\subsection{Reconstructing a Hitchin homomorphism from its invariants}

We now show how to reconstruct, up to conjugation by an element of $\operatorname{PSL}_{n}(\mathbb{R})$, a Hitchin homomorphism $\varrho: \pi_{1}(S) \rightarrow \mathrm{PSL}_{n}(\mathbb{R})$ from its triangle invariants and its shearing cycle.

For this, we first normalize $\varrho$ to avoid having to worry about conjugations. Fix a component $T_{0}$ of $\widetilde{S} \backslash \tilde{\lambda}$, with vertices $x_{0}, y_{0}, z_{0} \in \partial_{\infty} \widetilde{S}$. Also, choose a generic flag triple $\left(E_{0}, F_{0}, G_{0}\right)$.

Lemma 6.3. The flag triple $\left(E_{0}, F_{0}, G_{0}\right)$ can be chosen so that every Hitchin character is represented by a unique homomorphism $\varrho: \pi_{1}(S) \rightarrow \mathrm{PSL}_{n}(\mathbb{R})$ for which the flag $\mathcal{F}_{\varrho}\left(x_{0}\right)$ is equal to $E_{0}$, the flag $\mathcal{F}_{\varrho}\left(y_{0}\right)$ is equal to $F_{0}$, and the line $\mathcal{F}_{\varrho}\left(z_{0}\right)^{(1)}$ is equal to the line $G_{0}^{(1)}$. 
Note that the last condition involves only the line $\mathcal{F}_{\varrho}\left(z_{0}\right)^{(1)}$, not the whole flag $\mathcal{F}_{\varrho}\left(z_{0}\right)$.

Proof. For a Hitchin homomorphism $\varrho: \pi_{1}(S) \rightarrow \mathrm{PSL}_{n}(\mathbb{R})$, we know from Theorem 3.3 that the flag triple $\left(\mathcal{F}_{\varrho}\left(x_{0}\right), \mathcal{F}_{\varrho}\left(y_{0}\right), \mathcal{F}_{\varrho}\left(z_{0}\right)\right)$ is positive, and in particular generic. By elementary linear algebra, there consequently exists a unique projective isomorphism $\varphi_{\varrho} \in \mathrm{PGL}_{n}(\mathbb{R})$ sending the flag $\mathcal{F}_{\varrho}\left(x_{0}\right)$ to $E_{0}$, the flag $\mathcal{F}_{\varrho}\left(y_{0}\right)$ to $F_{0}$, and the line $\mathcal{F}_{\varrho}\left(z_{0}\right)^{(1)}$ to the line $G_{0}^{(1)}$. This $\varphi_{\varrho}$ depends continuously on $\varrho$.

If $\varphi_{\varrho_{0}} \in \mathrm{PGL}_{n}(\mathbb{R})$ is in $\mathrm{PSL}_{n}(\mathbb{R})$ for some Hitchin homomorphism $\varrho_{0}$ (which is automatic when $n$ is odd), then $\varphi_{\varrho}$ belongs to $\operatorname{PSL}_{n}(\mathbb{R})$ for all $\varrho$, by connectedness of the space of all Hitchin homomorphisms. Conjugating $\varrho$ by $\varphi_{\varrho} \in \mathrm{PSL}_{n}(\mathbb{R})$ replaces the flag curve $\mathcal{F}_{\varrho}: \partial_{\infty} \widetilde{S} \rightarrow \operatorname{Flag}\left(\mathbb{R}^{n}\right)$ by its composition with the action of $\varphi_{\varrho}$ on $\operatorname{Flag}\left(\mathbb{R}^{n}\right)$, and consequently provides a representative of the class of $\varrho$ in $\operatorname{Hit}_{n}(S)$ with the requested properties. The uniqueness of this representative is an immediate consequence of the fact that the stabilizer of $\left(E_{0}, F_{0}, G_{0}^{(1)}\right)$ in $\mathrm{PSL}_{n}(\mathbb{R})$ is trivial.

Otherwise, $\varphi_{\varrho}$ is in the complement of $\mathrm{PSL}_{n}(\mathbb{R})$ in $\mathrm{PGL}_{n}(\mathbb{R})$ for every Hitchin homomorphism $\varrho$. Replace the flag triple $\left(E_{0}, F_{0}, G_{0}\right)$ by its image under an arbitrary $\psi$ in this complement. This replaces each $\varphi_{\varrho}$ by $\psi \circ \varphi_{\varrho}$, which is now in $\operatorname{PSL}_{n}(\mathbb{R})$ for every Hitchin homomorphism. We then conclude as in the earlier case.

Note that all generic flag triples $\left(E_{0}, F_{0}, G_{0}\right)$ can be used in Lemma 6.3 when $n$ is odd, and "half" of them (namely those in one of the two equivalence classes of an appropriate equivalence relation) when $n$ is even.

The following lemma will help in the exposition, by decreasing the number of cases to consider. Let $g_{0}$ be the side of $T_{0}$ joining $x_{0}$ and $y_{0}$, and let $h_{0}$ be the side joining $x_{0}$ and $z_{0}$.

LEMMA 6.4. The fundamental group $\pi_{1}(S)$ is generated by finitely many elements $\gamma \in \pi_{1}(S)$ whose axes cross both $g_{0}$ and $h_{0}$, and send $T_{0}$ to a triangle $\gamma T_{0}$ contained in the component of $\widetilde{S} \backslash T_{0}$ that is adjacent to $g_{0}$, as in Figure 8.

Proof. The axes of $\pi_{1}(S)$ are dense in the space of geodesics of $\widetilde{S}$. Therefore, there exists an element $\gamma_{0} \in \pi_{1}(S)$ whose axis crosses both $g_{0}$ and $h_{0}$, and whose attracting fixed point in $\partial_{\infty} \widetilde{S}$ is contained in the closure of the component $U$ of $\widetilde{S} \backslash T_{0}$ delimited by $g_{0}$. In particular, $\gamma_{0} T_{0}$ is contained in $U$.

Let $\gamma_{1}, \gamma_{2}, \ldots, \gamma_{k}$ be a set of generators for $\pi_{1}(S)$. The ping-pong lemma shows that, for $m_{i}, n_{i}>0$ large enough, the attracting and repelling fixed points of $\gamma_{i}^{\prime}=\gamma_{0}^{m_{i}} \gamma_{i} \gamma_{0}^{n_{i}}$ are very close to the attracting and repelling fixed points of $\gamma_{0}$. In particular, the axis of $\gamma_{i}^{\prime}$ crosses both $g_{0}$ and $h_{0}$, and $\gamma_{i}^{\prime} T_{0}$ is contained in $U$. 

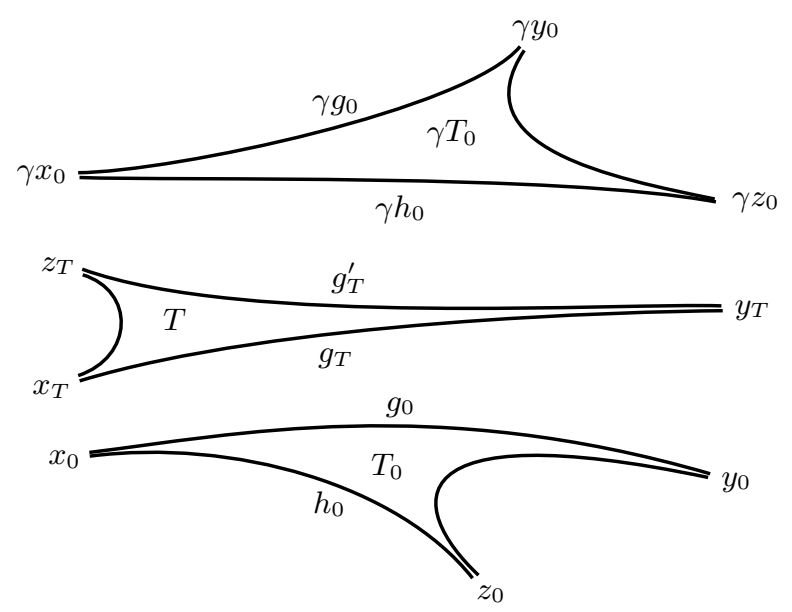

Figure 8. The triangles $T_{0}, \gamma T_{0}$ and $T$.

Then, the family of elements $\gamma_{0}, \gamma_{1}^{\prime}, \gamma_{2}^{\prime}, \ldots, \gamma_{k}^{\prime}$ generates $\pi_{1}(S)$ and has the required properties.

For a Hitchin homomorphism $\varrho: \pi_{1}(S) \rightarrow \operatorname{PSL}_{n}(\mathbb{R})$ and an element $\gamma \in \pi_{1}(S)$, the projective map $\varrho(\gamma) \in \operatorname{PSL}_{n}(\mathbb{R})$ sends the flag pair $\left(\mathcal{F}_{\varrho}\left(x_{0}\right), \mathcal{F}_{\varrho}\left(z_{0}\right)\right)$ to $\left(\mathcal{F}_{\varrho}\left(\gamma x_{0}\right), \mathcal{F}_{\varrho}\left(\gamma z_{0}\right)\right)$ by $\varrho$-equivariance of the flag map $\mathcal{F}_{\varrho}$. We would like to compare it to another map sending $\left(\mathcal{F}_{\varrho}\left(x_{0}\right), \mathcal{F}_{\varrho}\left(z_{0}\right)\right)$ to $\left(\mathcal{F}_{\varrho}\left(\gamma x_{0}\right), \mathcal{F}_{\varrho}\left(\gamma z_{0}\right)\right)$, namely the slithering map $\Sigma_{\left(\gamma h_{0}\right) h_{0}}=$ $\Sigma_{\left(\gamma h_{0}\right) g_{0}} \circ \Sigma_{g_{0} h_{0}}: \mathbb{R}^{n} \rightarrow \mathbb{R}^{n}$. Focusing on the side $g_{0}$ of the triangle $T_{0}$ instead of $h_{0}$, we

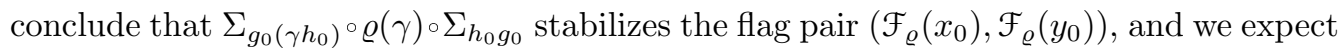
this element of the stabilizer to be related to the shearing vector $\sigma^{\varrho}\left(T_{0}, \gamma T_{0}\right) \in \mathbb{R}^{n-1}$.

With this in mind, we introduce a family of elements of the stabilizer of $\left(E_{0}, F_{0}\right)=$ $\left(\mathcal{F}_{\varrho}\left(x_{0}\right), \mathcal{F}_{\varrho}\left(y_{0}\right)\right)$, assuming $\varrho$ normalized as in Lemma 6.3. For $t=\left(t_{1}, t_{2}, \ldots, t_{n-1}\right) \in \mathbb{R}^{n-1}$, let $u_{1}, u_{2}, \ldots, u_{n}$ be uniquely determined by the properties that $t_{a}=u_{a}-u_{a+1}$ and $\sum_{a=1}^{n} u_{a}=0$. Namely,

$$
u_{a}=\frac{1}{n} \sum_{b=1}^{n-1}(n-b) t_{b}-\sum_{b=1}^{a-1} t_{b} .
$$

Then, let $\Theta_{E_{0} F_{0}}^{t}: \mathbb{R}^{n} \rightarrow \mathbb{R}^{n}$ be the element of $\mathrm{SL}_{n}(\mathbb{R})$ that acts by multiplication by $e^{u_{a}}$ on each line $E_{0}^{(a)} \cap F_{0}^{(n-a+1)}$.

We will also need to replace the slithering map $\Sigma_{g_{0} h_{0}}$ by another map $\Phi_{0} \in \mathrm{PGL}_{n}(\mathbb{R})$ that stabilizes the flag $\mathcal{F}_{\varrho}\left(x_{0}\right)$, and sends $\mathcal{F}_{\varrho}\left(z_{0}\right)$ to $\mathcal{F}_{\varrho}\left(y_{0}\right)$, but is normalized in such a way that

$$
D_{a}\left(\mathcal{F}_{\varrho}\left(x_{0}\right), \mathcal{F}_{\varrho}\left(y_{0}\right), \mathcal{F}_{\varrho}\left(z_{0}\right), \Phi_{0}\left(\mathcal{F}_{\varrho}\left(y_{0}\right)\right)\right)=1
$$

for every $a \in\{1,2, \ldots, n-1\}$. The existence and uniqueness of the projective map $\Phi_{0} \in$ $\mathrm{PGL}_{n}(\mathbb{R})$ is elementary. By comparison, the slithering map $\Sigma_{g_{0} h_{0}}$ was normalized so that 
it acts by the identity on each $\mathcal{F}_{\varrho}\left(x_{0}\right)^{(a+1)} / \mathcal{F}_{\varrho}\left(x_{0}\right)^{(a)} \cong \mathbb{R}$. The normalization defining $\Phi_{0}$ is better adapted to shearing parameters.

Lemma 6.5. Let $\varrho$ be normalized as in Lemma 6.3 and $\gamma \in \pi_{1}(S)$ be as in Lemma 6.4. Then,

$$
\varrho(\gamma)=\Sigma_{g_{0}\left(\gamma h_{0}\right)}^{-1} \circ \Theta_{E_{0} F_{0}}^{\sigma^{\varrho}\left(T_{0}, \gamma T_{0}\right)} \circ \Phi_{0} \in \mathrm{PGL}_{n}(\mathbb{R})
$$

where $\Theta_{E_{0} F_{0}}^{t}$ and $\Phi_{0}$ are defined as above, and where $\sigma^{\varrho}\left(T_{0}, \gamma T_{0}\right) \in \mathbb{R}^{n-1}$ is the shearing vector of $\varrho$ between $T_{0}$ and $\gamma T_{0}$ defined in $\S 5.2$.

Proof. Note that, since $\gamma$ satisfies the conclusions of Lemma 6.4, the side of $\gamma T_{0}$ that is closest to $T_{0}$ is $\gamma h_{0}$, while $g_{0}$ is the side of $T_{0}$ closest to $\gamma T_{0}$. See Figure 8.

The map $\Sigma_{g_{0}\left(\gamma h_{0}\right)} \varrho(\gamma)$ sends the flag triple $\left(\mathcal{F}_{\varrho}\left(x_{0}\right), \mathcal{F}_{\varrho}\left(z_{0}\right), \mathcal{F}_{\varrho}\left(y_{0}\right)\right)$ to

$$
\left(\mathcal{F}_{\varrho}\left(x_{0}\right), \mathcal{F}_{\varrho}\left(y_{0}\right), G_{0}^{\prime}\right)=\left(E_{0}, F_{0}, G_{0}^{\prime}\right),
$$

where $G_{0}^{\prime}=\Sigma_{g_{0}\left(\gamma h_{0}\right)}{ }^{\circ} \mathcal{F}_{\varrho}\left(\gamma y_{0}\right)$.

Similarly, $\Theta_{E_{0} F_{0}}^{\sigma^{\varrho}\left(T_{0}, \gamma T_{0}\right)} \circ \Phi_{0}$ sends $\left(\mathcal{F}_{\varrho}\left(x_{0}\right), \mathcal{F}_{\varrho}\left(z_{0}\right), \mathcal{F}_{\varrho}\left(y_{0}\right)\right)$ to

$$
\left(\mathcal{F}_{\varrho}\left(x_{0}\right), \mathcal{F}_{\varrho}\left(y_{0}\right), G_{0}^{\prime \prime}\right)=\left(E_{0}, F_{0}, G_{0}^{\prime \prime}\right),
$$

where $G_{0}^{\prime \prime}=\Theta_{E_{0} F_{0}}^{\sigma^{\varrho}\left(T_{0}, \gamma T_{0}\right)} \circ \Phi_{0} \circ \mathcal{F}_{\varrho}\left(y_{0}\right)$.

We want to compare the lines $G_{0}^{\prime(1)}$ and $G_{0}^{\prime \prime(1)}$. The key property is that

$$
\begin{aligned}
D_{a}\left(\mathcal{F}_{\varrho}\left(x_{0}\right), \mathcal{F}_{\varrho}\left(y_{0}\right), \mathcal{F}_{\varrho}\left(z_{0}\right), G_{0}^{\prime}\right) & =D_{a}\left(\mathcal{F}_{\varrho}\left(x_{0}\right), \mathcal{F}_{\varrho}\left(y_{0}\right), \mathcal{F}_{\varrho}\left(z_{0}\right), \Sigma_{g_{0}\left(\gamma h_{0}\right)}{ }^{\circ} \mathcal{F}_{\varrho}\left(\gamma y_{0}\right)\right) \\
& =e^{\sigma_{a}^{\varrho}\left(T_{0}, \gamma T_{0}\right)},
\end{aligned}
$$

by definition of the shearing parameter $\sigma_{a}^{\varrho}\left(T_{0}, \gamma T_{0}\right)$.

We have designed the definitions so that

$$
\begin{aligned}
\left.D_{a}\left(\mathcal{F}_{\varrho}\left(x_{0}\right), \mathcal{F}_{\varrho}\left(y_{0}\right), \mathcal{F}_{\varrho}\left(z_{0}\right), G_{0}^{\prime \prime}\right)\right) & =D_{a}\left(\mathcal{F}_{\varrho}\left(x_{0}\right), \mathcal{F}_{\varrho}\left(y_{0}\right), \mathcal{F}_{\varrho}\left(z_{0}\right), \Theta_{E_{0} F_{0}}^{\sigma^{\varrho}\left(T_{0}, \gamma T_{0}\right)} \circ \Phi_{0}\left(\mathcal{F}_{\varrho}\left(y_{0}\right)\right)\right) \\
& =e^{\sigma_{a}^{\varrho}\left(T_{0}, \gamma T_{0}\right)} D_{a}\left(\mathcal{F}_{\varrho}\left(x_{0}\right), \mathcal{F}_{\varrho}\left(y_{0}\right), \mathcal{F}_{\varrho}\left(z_{0}\right), \Phi_{0}\left(\mathcal{F}_{\varrho}\left(y_{0}\right)\right)\right) \\
& =e^{\sigma_{a}^{\varrho}\left(T_{0}, \gamma T_{0}\right)}
\end{aligned}
$$

where the second equality comes from Lemma 1.6 and from the definition of $\Theta_{E_{0} F_{0}}^{\sigma^{\varrho}\left(T_{0}, \gamma T_{0}\right)}$.

Comparing these two double ratios for every $a=1,2, \ldots, n$, we conclude that the lines $G_{0}^{\prime(1)}$ and $G_{0}^{\prime \prime(1)}$ are equal (use for instance Lemma 1.6 again).

Therefore, the two projective maps $\Sigma_{g_{0}\left(\gamma h_{0}\right)}{ }^{\circ} \varrho(\gamma)$ and $\Theta_{E_{0} F_{0}}^{\sigma^{\varrho}\left(T_{0}, \gamma T_{0}\right)} \circ \Phi_{0}$ send the flag $\mathcal{F}_{\varrho}\left(x_{0}\right)$ to the same flag $\mathcal{F}_{\varrho}\left(x_{0}\right)$, send the flag $\mathcal{F}_{\varrho}\left(z_{0}\right)$ to the same flag $\mathcal{F}_{\varrho}\left(y_{0}\right)$, and send the line $\mathcal{F}_{\varrho}\left(y_{0}\right)^{(1)}$ to the same line $G_{0}^{\prime(1)}=G_{0}^{\prime \prime(1)}$. By genericity of the flag triple $\left(\mathcal{F}_{\varrho}\left(x_{0}\right), \mathcal{F}_{\varrho}\left(z_{0}\right), \mathcal{F}_{\varrho}\left(y_{0}\right)\right)$ they consequently coincide, which proves the lemma. 
In the formula of Lemma 6.5, the term $\Theta_{E_{0} F_{0}}^{\sigma^{\varrho}\left(T_{0}, \gamma T_{0}\right)}$ depends only on the shearing cycle $\sigma^{\varrho}$. The term $\Phi_{0}$ is defined by the generic flag triple $\left(\mathcal{F}_{\varrho}\left(x_{0}\right), \mathcal{F}_{\varrho}\left(z_{0}\right), \mathcal{F}_{\varrho}\left(y_{0}\right)\right)$ and, because of our normalization that $\mathcal{F}_{\varrho}\left(x_{0}\right)=E_{0}, \mathcal{F}_{\varrho}\left(y_{0}\right)=F_{0}$ and $\mathcal{F}_{\varrho}\left(z_{0}\right)^{(1)}=G_{0}^{(1)}$, is completely determined by the triangle invariants $\tau_{a b c}^{\varrho}(s)$ of the base triangle $T_{0}$. We now turn our attention to the remaining term, the slithering map $\Sigma_{g_{0}\left(\gamma h_{0}\right)}$.

By Proposition 6.2,

$$
\Sigma_{g_{0}\left(\gamma h_{0}\right)}=\coprod_{T \in \mathcal{T}_{g_{0}\left(\gamma h_{0}\right)}} \widehat{\Sigma}_{T}
$$

with the notation of that statement.

Consider the contribution $\widehat{\Sigma}_{T}=\Sigma_{g_{0} g_{T}} \circ \Sigma_{T} \circ \Sigma_{g_{0} g_{T}}^{-1}$ of a triangle $T \in \mathcal{T}_{g_{0}\left(\gamma h_{0}\right)}$, separating $g_{0}$ from $\gamma h_{0}$.

LEMma 6.6. For each triangle $T \in \mathcal{T}_{g_{0}\left(\gamma h_{0}\right)}$, the map $\widehat{\Sigma}_{T} \in \mathrm{PSL}_{n}(\mathbb{R})$ is uniquely determined by the shearing vector $\sigma^{\varrho}\left(T_{0}, T\right) \in \mathbb{R}^{n-1}$ and by the triangle invariants $\tau_{\text {abc }}^{\varrho}(s) \in \mathbb{R}$ of the triangle $T$.

More precisely,

$$
\widehat{\Sigma}_{T}=\Theta^{\sigma^{\varrho}\left(T_{0}, T\right)} \circ \widehat{\Sigma}_{T}^{\prime} \circ \Theta^{-\sigma^{\varrho}\left(T_{0}, T\right)}
$$

where $\widehat{\Sigma}_{T}^{\prime}$ depends only on the triangle invariants $\tau_{a b c}^{\varrho}(s)$ of $T$.

Proof. Index the vertices of $T$ as $x_{T}, y_{T}$ and $z_{T}$, in such a way that the side $g_{T}=y_{T} x_{T}$ is the one that is closest to $g_{0}=y_{0} x_{0}$, and is oriented in parallel with $g_{0}$. See Figure 8 .

As in the definition of the map $\Phi_{0}$, there is a unique projective map $\Phi_{T} \in \mathrm{PGL}_{n}(\mathbb{R})$ sending $\mathcal{F}_{\varrho}\left(x_{T}\right)$ to $\mathcal{F}_{\varrho}\left(x_{0}\right)=E_{0}, \mathcal{F}_{\varrho}\left(y_{T}\right)$ to $\mathcal{F}_{\varrho}\left(y_{0}\right)=F_{0}$, and $\mathcal{F}_{\varrho}\left(z_{T}\right)$ to a flag $G_{T}^{\prime}$ such that

$$
D_{a}\left(\mathcal{F}_{\varrho}\left(x_{0}\right), \mathcal{F}_{\varrho}\left(y_{0}\right), \mathcal{F}_{\varrho}\left(z_{0}\right), G_{T}^{\prime}\right)=1
$$

for every $a=1,2, \ldots, n$.

A fundamental observation is that, although the map $\Phi_{T}$ depends on the flag triple $\left(\mathcal{F}_{\varrho}\left(x_{T}\right), \mathcal{F}_{\varrho}\left(y_{T}\right), \mathcal{F}_{\varrho}\left(z_{T}\right)\right)$, the flag $G_{T}^{\prime}=\Phi_{T}\left(\mathcal{F}_{\varrho}\left(z_{T}\right)\right)$ depends only on the orbit of that flag triple under the action of $\mathrm{PGL}_{n}(\mathbb{R})$ or, equivalently, on the triangle invariants $\tau_{a b c}^{\varrho}(s)$ of the triangle $T$.

The two maps $\Phi_{T}$ and $\Sigma_{g_{0} g_{T}}$ both send $\mathcal{F}_{\varrho}\left(x_{T}\right)$ to $\mathcal{F}_{\varrho}\left(x_{0}\right)$ and $\mathcal{F}_{\varrho}\left(y_{T}\right)$ to $\mathcal{F}_{\varrho}\left(y_{0}\right)$. As in the proof of Lemma 6.5,

$$
D_{a}\left(\mathcal{F}_{\varrho}\left(x_{0}\right), \mathcal{F}_{\varrho}\left(y_{0}\right), \mathcal{F}_{\varrho}\left(z_{0}\right), \Sigma_{g_{0} g_{T}}\left(\mathcal{F}_{\varrho}\left(z_{T}\right)\right)=e^{\sigma_{a}\left(T_{0}, T\right)}\right.
$$

by definition of the shearing parameter $\sigma_{a}\left(T_{0}, T\right)$, and

$$
D_{a}\left(\mathcal{F}_{\varrho}\left(x_{0}\right), \mathcal{F}_{\varrho}\left(y_{0}\right), \mathcal{F}_{\varrho}\left(z_{0}\right), \Theta_{E_{0} F_{0}}^{\sigma^{\varrho}\left(T_{0}, T\right)} \circ \Phi_{T}\left(\mathcal{F}_{\varrho}\left(z_{T}\right)\right)\right)=e^{\sigma_{a}\left(T_{0}, T\right)}
$$


by construction. Again as in the proof of Lemma 6.5, it follows that

$$
\Sigma_{g_{0} g_{T}}=\Theta_{E_{0} F_{0}}^{\sigma^{\varrho}\left(T_{0}, T\right)} \circ \Phi_{T} .
$$

Noting that $\left(\Theta_{E_{0} F_{0}}^{t}\right)^{-1}=\Theta_{E_{0} F_{0}}^{-t}$, we set

$$
\begin{aligned}
\widehat{\Sigma}_{T}^{\prime} & =\Theta_{E_{0} F_{0}}^{-\sigma^{\varrho}\left(T_{0}, T\right)} \circ \widehat{\Sigma}_{T} \circ \Theta_{E_{0} F_{0}}^{\sigma^{\varrho}\left(T_{0}, T\right)} \\
& =\Theta_{E_{0} F_{0}}^{-\sigma^{\varrho}\left(T_{0}, T\right)} \circ \Sigma_{g_{0} g_{T}} \circ \Sigma_{T} \circ \Sigma_{g_{0} g_{T}}^{-1} \circ \Theta_{E_{0} F_{0}}^{\sigma^{\varrho}\left(T_{0}, T\right)}=\Phi_{T} \circ \Sigma_{T} \circ \Phi_{T}^{-1} .
\end{aligned}
$$

There are now two cases to distinguish in the exposition, according to whether the side $g_{T}^{\prime}$ of $T$ that is closest to $\gamma T_{0}$ is equal to $z_{T} x_{T}$ or to $y_{T} z_{T}$.

Consider the case where $T$ points to the right, namely where $g_{T}^{\prime}$ is equal to $y_{T} z_{T}$, as in Figure 8. Then, the elementary slithering $\Sigma_{T}=\Sigma_{g_{T} g_{T}^{\prime}}$ is the unique linear map that fixes the flag $\mathcal{F}_{\varrho}\left(y_{T}\right)$, acts by the identity on each line $\mathcal{F}_{\varrho}\left(y_{T}\right)^{(a+1)} / \mathcal{F}_{\varrho}\left(y_{T}\right)^{(a)}$, and sends the flag $\mathcal{F}_{\varrho}\left(z_{T}\right)$ to $\mathcal{F}_{\varrho}\left(x_{T}\right)$. It follows that $\widehat{\Sigma}_{T}^{\prime}=\Phi_{T} \circ \Sigma_{T} \circ \Phi_{T}^{-1}$ is the unique linear map that fixes the flag $\Phi_{T}\left(\mathcal{F}_{\varrho}\left(y_{T}\right)\right)=F_{0}$, acts as the identity on each line $F_{0}^{(a+1)} / F_{0}^{(a)}$, and sends the flag $\Phi_{T}\left(\mathcal{F}_{\varrho}\left(z_{T}\right)\right)=G_{T}^{\prime}$ to $\Phi_{T}\left(F_{\varrho}\left(x_{T}\right)\right)=E_{0}$. Since we already observed that the flag $G_{T}^{\prime}$ is uniquely determined by the triangle invariants $\tau_{a b c}^{\varrho}(s)$, so is the map $\widehat{\Sigma}_{T}^{\prime}$ and

$$
\widehat{\Sigma}_{T}=\Theta^{\sigma^{\varrho}\left(T_{0}, T\right)} \circ \widehat{\Sigma}_{T}^{\prime} \circ \Theta^{-\sigma^{\varrho}\left(T_{0}, T\right)}
$$

depends only on these triangle invariants as well as the shearing vector $\sigma^{\varrho}\left(T_{0}, T\right)$.

The argument is essentially identical in the case where the triangle $T$ points to the left, namely when $g_{t}^{\prime}$ is equal to the geodesic $z_{T} x_{T}$. In this case, $\widehat{\Sigma}_{T}^{\prime}$ is the unique linear map that fixes $E_{0}$, acts as the identity on each line $E_{0}^{(a+1)} / E_{0}^{(a)}$, and sends $G_{T}^{\prime}$ to $F_{0}$. Again, this proves that $\widehat{\Sigma}_{T}^{\prime}$ is uniquely determined by the triangle invariants $\tau_{a b c}^{\varrho}(s)$.

Lemma 6.7. Let $\varrho$ be normalized as in Lemma 6.3 and $\gamma \in \pi_{1}(S)$ be as in Lemma 6.4. Then,

$$
\varrho(\gamma)=\left(\prod_{T \in \mathcal{T}_{g_{0}\left(\gamma h_{0}\right)}}^{\overleftarrow{T_{0}}}\left(\Theta_{E_{0} F_{0}}^{\sigma^{\varrho}\left(T_{0}, T\right)} \circ \widehat{\Sigma}_{T}^{\prime} \circ \Theta_{E_{0} F_{0}}^{-\sigma^{\varrho}\left(T_{0}, T\right)}\right)\right)^{-1} \circ \Theta_{E_{0} F_{0}}^{\sigma^{\varrho}\left(T_{0}, \gamma T_{0}\right)} \circ \Phi_{0}
$$

in $\mathrm{PGL}_{n}(\mathbb{R})$, with the definitions introduced above. In particular, the maps $\widehat{\Sigma}_{T}^{\prime}$ and $\Phi_{0}$ depend only on the triangle invariants $\tau_{a b c}^{\varrho}(s)$ of $\varrho$, while the terms $\Theta_{E_{0} F_{0}}^{ \pm \sigma_{0}}\left(_{0}, T\right)$ are determined by its shearing cycle $\sigma^{\varrho} \in \mathcal{C}\left(\lambda\right.$, slits; $\left.\widehat{\mathbb{R}}^{n-1}\right)$.

Proof. This follows from the combination of Lemmas 6.5 and 6.6, together with the observation that $\Phi_{0}$ depends only on the triangle invariants $\tau_{a b c}^{\varrho}$.

COROllary 6.8. Two Hitchin homomorphisms $\varrho, \varrho^{\prime}: \pi_{1}(S) \rightarrow \mathrm{PSL}_{n}(\mathbb{R})$ that have the same triangle invariants $\sigma_{a b c}^{\varrho}(s)=\sigma_{a b c}^{\varrho^{\prime}}(s)$ and the same shearing cycles $\sigma^{\varrho}=\sigma^{\varrho^{\prime}} \in$ $\mathcal{C}\left(\lambda\right.$, slits; $\left.\widehat{\mathbb{R}}^{n}\right)$ are conjugate by an element of $\operatorname{PSL}_{n}(\mathbb{R})$, and therefore represent the same character in $\operatorname{Hit}_{n}(S)$. 
Proof. Conjugate $\varrho$ and $\varrho^{\prime}$ by elements of $\operatorname{PSL}_{n}(\mathbb{R})$ in order to normalize them as in Lemma 6.3. Then, for every element $\gamma \in \pi_{1}(S)$ satisfying the conditions of Lemma 6.4, the formula of Lemma 6.7 shows that $\varrho(\gamma)=\varrho^{\prime}(\gamma)$. Since these $\gamma$ generate $\pi_{1}(S)$, this proves that $\varrho=\varrho^{\prime}$.

\section{Length functions}

Our next goal is to determine which triangle invariants and shearing cycles can be realized as invariants of Hitchin characters. The length functions considered in this section provide one of the constraints that need to be satisfied by these invariants.

\subsection{Length functions associated with Hitchin characters}

Let $\varrho: \pi_{1}(S) \rightarrow \mathrm{PSL}_{n}(\mathbb{R})$ be a Hitchin homomorphism. Labourie proves in [27] that, for every non-trivial $\gamma \in \pi_{1}(S)$, the matrix $\varrho(\gamma) \in \mathrm{PSL}_{n}(\mathbb{R})$ is diagonalizable and its eigenvalues can be indexed as $\mu_{1}(\varrho(\gamma)), \mu_{2}(\varrho(\gamma)), \ldots, \mu_{n}(\varrho(\gamma))$ in such a way that

$$
\frac{\mu_{a}(\varrho(\gamma))}{\mu_{a+1}(\varrho(\gamma))}>1
$$

for every $i=1,2, \ldots, n-1$. (Note that eigenvalues of an element of $\mathrm{PSL}_{n}(\mathbb{R})$ are only defined up to sign when $n$ is even, but that the quotient between two such eigenvalues makes intrinsic sense.) This property is in fact an easy consequence of Theorem 3.1.

Eigenvalues are invariant under conjugation. This consequently defines $n-1$ functions

$$
\ell_{a}^{\varrho}:\left\{\text { non-trivial conjugacy classes of } \pi_{1}(S)\right\} \longrightarrow \mathbb{R}
$$

by the property that

$$
\ell_{a}^{\varrho}(\gamma)=\log \frac{\mu_{a}(\varrho(\gamma))}{\mu_{a+1}(\varrho(\gamma))}>0 .
$$

The same conjugation invariance shows that the length function $\ell_{a}^{\varrho}$ depends only on the Hitchin character $\varrho \in \operatorname{Hit}_{n}(S)$, not on the Hitchin homomorphism $\varrho: \pi_{1}(S) \rightarrow \mathrm{PSL}_{n}(\mathbb{R})$ that represents it.

The set of conjugacy classes of the fundamental group $\pi_{1}(S)$ is discrete, but these length functions have a natural extension to a continuous space. Indeed, endowing the surface $S$ with an arbitrary negatively curved riemannian metric, a conjugacy class of $\pi_{1}(S)$ uniquely determines an oriented closed geodesic of $S$, and therefore a closed orbit of the geodesic flow of the unit tangent bundle $T^{1} S$. This closed leaf is endowed with an integer weight $m>0$ if the conjugacy class is an $m$-power of a primitive class. Considering 
the Dirac transverse measure defined by this closed orbit and this weight, this provides an analytic interpretation of a conjugacy class of $\pi_{1}(S)$ as a transverse measure for the geodesic foliation $\mathcal{F}_{S}$ of $T^{1} S$, whose leaves are the orbits of the geodesic flow.

This defines a completion of the set of conjugacy classes of $\pi_{1}(S)$ by the space $\mathrm{e}^{\mathrm{m}}(S)$ of all (positive Radon) transverse measures for the geodesic foliation $\mathcal{F}_{S}$ [2], [3], [4], analogous to Thurston's completion [39], [17], [36] of the set of isotopy classes of simple closed curves in $S$ by the space $\mathcal{M} \mathcal{L}(S)$ of measured laminations on $S$.

For differentiability properties, it is useful to consider more general transverse structures for the geodesic foliation, namely transverse Hölder distributions in the sense of [7] and [6]. When the geodesic foliation $\mathcal{F}_{S}$ is realized by the geodesic flow of a riemannian metric $m$ of constant negative curvature, it inherits from that construction a transverse differential structure, namely a smooth structure on the space of leaves of $\mathcal{F}_{S} \cap U$ for every sufficiently small open subset of $T^{1} S$. A transverse Hölder distribution then is a distribution, in the usual sense, on these local leaf spaces with additional regularity properties that makes the notion independent of the auxiliary riemannian metric $m$. See [7] and [6] for details.

In particular, a transverse measure is a special type of transverse Hölder distribution. This embeds the set of conjugacy classes of $\pi_{1}(S)$ in the topological vector space $\mathrm{e}^{\mathrm{H}}(S)$ of all transverse Hölder distributions for the geodesic foliation $\mathcal{F}_{S}$. In other words, we now have embeddings

$$
\left\{\text { non-trivial conjugacy classes of } \pi_{1}(S)\right\} \subset \mathrm{e}^{\mathrm{m}}(S) \subset \mathrm{e}^{\mathrm{H}}(S) \text {. }
$$

The elements of $\mathrm{e}^{\mathrm{m}}(S)$ and $\mathrm{e}^{\mathrm{H}}(S)$ are respectively called measure geodesic currents and Hölder geodesic currents for the surface $S$. See the references mentioned above for a proof that these constructions depend only on the topology of the surface $S$.

THEOREM 7.1. ([15]) For each Hitchin character $\varrho \in \operatorname{Hit}_{n}(S)$ and for each $a=1,2, \ldots$, $n-1$, the length function

$$
\ell_{a}^{\varrho}:\left\{\text { non-trivial conjugacy classes of } \pi_{1}(S)\right\} \longrightarrow \mathbb{R}
$$

extends to a continuous linear map $\ell_{a}^{\varrho}: \mathrm{e}^{\mathrm{H}}(S) \rightarrow \mathbb{R}$.

Remark 7.2. The reader should beware that the above functions $\ell_{a}^{\varrho}$ are slightly different from those introduced in [15]. Namely, our functions $\ell_{a}^{\varrho}$ would be called $\ell_{a}^{\varrho}-\ell_{a+1}^{\varrho}$ in [15]. Although mathematically equivalent to those of [15], our conventions are better adapted to the framework of the current article, as will be apparent in Proposition 7.4 and Theorem 7.5 below. 
Remark 7.3. By linearity and continuity, the extension $\ell_{a}^{\varrho}: \mathrm{e}^{\mathrm{H}}(S) \rightarrow \mathbb{R}$ is uniquely determined on the closure of the set of all linear combinations of conjugacy classes of $\pi_{1}(S)$. We do not know if this closure is equal to all of $\mathrm{e}^{\mathrm{H}}(S)$ (this seems unlikely), but it can be shown to contain all the Hölder geodesic currents that we will encounter in this article, including the image of the embedding $z(\hat{\lambda} ; \mathbb{R}) \rightarrow \mathrm{e}^{\mathrm{H}}(S)$ that will appear in $\S 7.2$.

The following statement will be particularly important in our characterization of which relative tangent cycles can occur as shearing cycles of Hitchin characters.

Proposition 7.4. Let $\alpha \in \mathrm{C}^{\mathrm{m}}(S)$ be a non-zero measure geodesic current. Then,

$$
\ell_{a}^{\varrho}(\alpha)>0
$$

for every Hitchin character $\varrho \in \operatorname{Hit}_{n}(S)$ and every $a=1,2, \ldots, n-1$.

Proof. This is a simple consequence of the Anosov property of Theorem 3.1.

For this, we need to remind the reader of the construction of the length functions $\ell_{a}^{\varrho}: \mathrm{e}^{\mathrm{H}}(S) \rightarrow \mathbb{R}$ in [15], taking Remark 7.2 into account. As in $\S 3.1$, consider the geodesic flow $\left(g_{t}\right)_{t \in \mathbb{R}}$ on the unit tangent bundle $T^{1} S$ (for an arbitrary metric of negative curvature) and its flat lift to a flow $\left(G_{t}\right)_{t \in \mathbb{R}}$ on the vector bundle $T^{1} S \times{ }_{\varrho^{\prime}} \mathbb{R}^{n}$, twisted by a homomorphism $\varrho^{\prime}: \pi_{1}(S) \rightarrow \mathrm{SL}_{n}(\mathbb{R})$ lifting $\varrho$. In addition, choose a riemannian metric $\|\cdot\|$ on the vector bundle $T^{1} S \times{ }_{\varrho^{\prime}} \mathbb{R}^{n} \rightarrow T^{1} S$.

The vector bundle $T^{1} S \times{ }_{\varrho^{\prime}} \mathbb{R}^{n} \rightarrow T^{1} S$ splits as a direct sum of line bundles $L_{a} \rightarrow T^{1} S$ as in $\S 3.1$. For $a=1,2, \ldots, n$, this data provides a function $f_{a}: T^{1} S \rightarrow \mathbb{R}$ defined by the property that, for $x \in T^{1} S$,

$$
f_{a}(x)=-\left.\left(\frac{d}{d t} \log \left\|G_{t}\left(v_{a}(x)\right)\right\|_{g_{t}(x)}\right)\right|_{t=0},
$$

where $v_{a}(x)$ is an arbitrary non-zero vector in the fiber $L_{a}(x)$ of the line bundle $L_{a} \rightarrow T^{1} S$. For a measure geodesic current $\alpha \in \mathcal{C}^{\mathrm{m}}(S)$, the length $\ell_{a}^{\varrho}(\alpha)$ is then defined as the integral

$$
\ell_{a}^{\varrho}(\alpha)=\int_{T^{1} S}\left(f_{a}-f_{a+1}\right) \alpha \times d t
$$

of the function $f_{a}-f_{a+1}$ with respect to the measure $\alpha \times d t$ on $T^{1} S$ that, locally, is the product of the transverse measure $\alpha$ for the geodesic flow $\left(g_{t}\right)_{t \in \mathbb{R}}$ with the measure $d t$ along the orbits of this geodesic flow. 
The measure $\alpha \times d t$ is invariant under the geodesic flow. Therefore, for every $t_{0}>0$,

$$
\begin{aligned}
\int_{T^{1} S} f_{a} \alpha \times d t & =\int_{T^{1} S} f_{a} \circ g_{u} \alpha \times d t \\
& =\frac{1}{t_{0}} \int_{T^{1} S} \int_{0}^{t_{0}} f_{a} \circ g_{u} d u \alpha \times d t \\
& =\frac{1}{t_{0}} \int_{T^{1} S} \int_{0}^{t_{0}}-\frac{d}{d u} \log \left\|G_{u}\left(v_{a}(x)\right)\right\|_{g_{u}(x)} d u \alpha \times d t(x) \\
& =\frac{1}{t_{0}} \int_{T^{1} S} \log \frac{\left\|v_{a}(x)\right\|_{x}}{\left\|G_{t_{0}}\left(v_{a}(x)\right)\right\|_{g_{t_{0}}(x)}} \alpha \times d t(x),
\end{aligned}
$$

so that

$$
\ell_{a}^{\varrho}(\alpha)=\frac{1}{t_{0}} \int_{T^{1} S} \log \frac{\left\|v_{a}(x)\right\|_{x}}{\left\|G_{t_{0}}\left(v_{a}(x)\right)\right\|_{g_{t_{0}}(x)}} \frac{\left\|G_{t_{0}}\left(v_{a+1}(x)\right)\right\|_{g_{t_{0}}(x)}}{\left\|v_{a+1}(x)\right\|_{x}} \alpha \times d t(x) .
$$

Theorem 3.1 provides constants $A, B>0$ such that

$$
\log \frac{\left\|v_{a}(x)\right\|_{x}}{\left\|G_{t_{0}}\left(v_{a}(x)\right)\right\|_{g_{t_{0}}(x)}} \frac{\left\|G_{t_{0}}\left(v_{a+1}(x)\right)\right\|_{g_{t_{0}}(x)}}{\left\|v_{a+1}(x)\right\|_{x}} \geqslant \log A+B t_{0}
$$

for every $t_{0}>0$. In particular, this integrand is strictly positive for $t_{0}$ large enough, and it follows that the integral $\ell_{a}^{\varrho}(\alpha)$ is strictly positive.

\subsection{Shearing cycles and length functions}

We now consider a special type of Hölder geodesic current.

We saw in $\S 4.1$ that a positive tangent cycle $\mu \in \mathcal{Z}(\hat{\lambda} ; \mathbb{R})$ determines a transverse measure for $\hat{\lambda}$. A general tangent cycle $\alpha \in Z(\hat{\lambda} ; \mathbb{R})$ determines a transverse Hölder distribution, which lifts to a Hölder geodesic current $\alpha \in \mathrm{e}^{\mathrm{H}}(S)$ [7], [6]. This provides an embedding $\mathcal{Z}(\hat{\lambda} ; \mathbb{R}) \subset \mathrm{e}^{\mathrm{H}}(S)$, and the length functions $\ell_{a}^{\varrho}: \mathrm{e}^{\mathrm{H}}(S) \rightarrow \mathbb{R}$ of the previous section restrict to linear functions $\ell_{a}^{\varrho}: z(\hat{\lambda} ; \mathbb{R}) \rightarrow \mathbb{R}$.

The following statement connects these length functions to the homological interpretation of relative tangent cycles in $\S 4.5$, based on a train-track neighborhood $\widehat{U}$ of the orientation cover $\hat{\lambda}$. Proposition 4.5 provides isomorphisms $z(\hat{\lambda}$, slits; $\mathbb{R}) \cong H_{1}\left(\widehat{U}, \partial_{\mathrm{v}} \widehat{U} ; \mathbb{R}\right)$ and $z(\hat{\lambda} ; \mathbb{R}) \cong H_{1}(\widehat{U} ; \mathbb{R})$.

THEOREM 7.5. Let $\varrho \in \operatorname{Hit}_{n}(S)$ be a Hitchin character with shearing cyle

$$
\sigma^{\varrho} \in Z\left(\lambda, \text { slits } ; \widehat{\mathbb{R}}^{n-1}\right) \subset Z\left(\hat{\lambda}, \text { slits; } \mathbb{R}^{n-1}\right) .
$$

Then, for every $a=1,2, \ldots, n-1$, the a-th component $\sigma_{a}^{\varrho} \in Z(\hat{\lambda}, \operatorname{slits} ; \mathbb{R}) \cong H_{1}\left(\widehat{U}, \partial_{\mathrm{v}} \widehat{U} ; \mathbb{R}\right)$ of $\sigma^{\varrho}$ is related to the length function $\ell_{a}^{\varrho}: \mathcal{Z}(\hat{\lambda} ; \mathbb{R}) \rightarrow \mathbb{R}$ by the property that

$$
\ell_{a}^{\varrho}(\alpha)=[\alpha] \cdot\left[\sigma_{a}^{\varrho}\right]
$$


for every tangent cycle $\alpha \in Z(\hat{\lambda} ; \mathbb{R}) \cong H_{1}(\widehat{U} ; \mathbb{R})$, where $\cdot$ denotes the algebraic intersection number of relative homology classes in the train-track neighborhood $\widehat{U}$ of the orientation cover $\hat{\lambda}$.

In the case where $n=2$, this statement is implicit in [42], and explicitly expressed in $[5$, Theorem $\mathrm{E}]$ in terms of the Thurston intersection form on the space $z(\lambda ; \mathbb{R})$ of tangent cycles.

We will split the proof of Theorem 7.5 into several lemmas.

We first give a homological interpretation of the length function $\ell_{a}^{\varrho}: z(\hat{\lambda} ; \mathbb{R}) \rightarrow \mathbb{R}$, by connecting it to a certain closed 1-form $\Omega_{a}$ defined on $\widehat{U}$. Because of the lack of transverse regularity of the geodesic lamination $\hat{\lambda}$ and of the line bundles $L_{a} \rightarrow T^{S}$, we however need a version of closed 1-form that is weaker than the usual differentiable definition.

\subsubsection{Topological closed 1-forms}

Remember that a differential form is closed if and only if it is locally exact. This motivates the following definition. A topological closed 1 -form $\Omega$ on a topological space $X$ is defined by a family $\left\{\left(V_{i}, F_{i}\right)\right\}_{i \in I}$ such that the following conditions hold:

(1) each $V_{i}$ is open in $X$, and $X=\bigcup_{i \in I} V_{i}$;

(2) each $F_{i}: V_{i} \rightarrow \mathbb{R}$ is a continuous function;

(3) for every $i, j \in I$, the function $F_{i}-F_{j}$ is locally constant on $V_{i} \cap V_{j}$.

By definition, two such families $\left\{\left(V_{i}, F_{i}\right)\right\}_{i \in I}$ and $\left\{\left(V_{i^{\prime}}^{\prime}, F_{i^{\prime}}^{\prime}\right)\right\}_{i^{\prime} \in I^{\prime}}$ define the same topological closed 1-form when, for every $i \in I$ and $i^{\prime} \in I^{\prime}$, the function $F_{i}-F_{i^{\prime}}^{\prime}$ is locally constant on $V_{i} \cap V_{i^{\prime}}^{\prime}$. In other words, a topological closed 1 -form $\Omega$ is defined as an equivalence class of families $\left\{\left(V_{i}, F_{i}\right)\right\}_{i \in I}$ satisfying the above three conditions. Equivalently, a topological closed 1-form is a maximal family $\left\{\left(V_{i}, F_{i}\right)\right\}_{i \in I}$ satisfying the above three conditions.

The motivation of the definition comes of course from the case where $X$ is a differentiable manifold and all functions $F_{i}$ are differentiable. Such a family of charts $\left\{\left(V_{i}, F_{i}\right)\right\}_{i \in I}$ uniquely determines a closed differential 1-form $\Omega$ by the property that $\left.\Omega\right|_{V_{i}}=d F_{i}$ on each chart $V_{i} \subset X$.

The definition of a topological closed 1 -form $\Omega$ is specially designed so that we can integrate $\Omega$ over every oriented continuous arc $k$. Indeed, if $\Omega$ is defined by a family of charts $\left\{\left(V_{i}, F_{i}\right)\right\}_{i \in I}$, the arc $k$ can be split into a finite family of arcs $k_{1}, k_{2}, \ldots, k_{p}$ such that each $k_{j}$ is contained in some chart $V_{i_{j}}$. Then, the integral of $\Omega$ over the arc $k$ is defined as

$$
\int_{k} \Omega=\sum_{j=1}^{p}\left(F_{i_{j}}\left(x_{j}^{+}\right)-F_{i_{j}}\left(x_{j}^{-}\right)\right),
$$


where $x_{j}^{+}, x_{j}^{-} \in V_{i_{j}}$ are the positive and negative endpoints of the arc $k_{j}$. It is immediate that this definition is independent of the decomposition of $k$ into subarcs $k_{j}$ (and of the chart $V_{i_{j}}$ containing each individual $k_{j}$ ), and that the integral $\int_{k} \Omega$ is invariant under homotopy of $k$ fixing its endpoints.

The construction is of course designed to coincide with the usual definition when $\Omega$ is a closed differential 1 -form and the arc $k$ is differentiable.

The invariance of the integral $\int_{k} \Omega$ under homotopy of $k$ fixing endpoints yields the following result.

Lemma 7.6. A topological closed 1-form $\Omega$ on the space $X$ defines a cohomology class $[\Omega] \in H^{1}(X ; \mathbb{R})$, represented by the singular cochain assigning the integral $\int_{k} \Omega$ to each continuous arc (= singular 1-simplex) $k$.

When $X$ is a metric space, we say that a topological closed 1 -form $\Omega$ is Hölder continuous if it can be defined by a family of charts $\left\{\left(V_{i}, F_{i}\right)\right\}_{i \in I}$, where each function $F_{i}: V_{i} \rightarrow \mathbb{R}$ is Hölder continuous. The terminology is convenient but somewhat abusive, because such a form is only, locally, the "differential" of a Hölder continuous function.

\subsubsection{A homological interpretation of the lengths of tangent cycles}

The length functions $\ell_{a}^{\varrho}$ are constructed in [15] by using the differential 1-forms $\omega_{a}=f_{a} d t$, defined on the orbits of the geodesic flow in the unit tangent bundle $T^{1} S$, that we encountered in the proof of Proposition 7.4.

The (geodesic) leaves of $\hat{\lambda}$ have canonical lifts to orbits of the geodesic flow in $T^{1} S$, so that the 1 -forms $\omega_{a}$ induce 1 -forms along the leaves of $\hat{\lambda}$, which we will also denote by $\omega_{a}$. We want to extend the forms $\omega_{a}$ to the train-track neighborhood $\widehat{U}$ of $\hat{\lambda}$.

We first extend $\omega_{a}$ to the subset $\hat{\lambda}$.

Lemma 7.7. There exists a Hölder continuous topological closed 1-form $\Omega_{a}$ on the orientation cover $\hat{\lambda}$ whose restriction to each leaf of $\hat{\lambda}$ is equal to the form $\omega_{a}=f_{a} d t$ above.

Proof. We need to remember the precise definition of the form $\omega_{a}$. We fix a riemannian metric $\|\cdot\|$ on the flat bundle $T^{1} S \widetilde{\times}_{\varrho^{\prime}} \mathbb{R}^{n}$, where $\varrho^{\prime}: \pi_{1}(S) \rightarrow \mathrm{SL}_{n}(\mathbb{R})$ lifts the Hitchin homomorphism $\varrho: \pi_{1}(S) \rightarrow \operatorname{PSL}_{n}(\mathbb{R})$. Each $x \in \hat{\lambda}$ determines a point $(x, v)$ in the unit tangent bundle $T^{1} S$ by considering the unit tangent vector $v \in T_{x}^{1} S$ of the (oriented) leaf of $\hat{\lambda}$ passing through $x$. This enables us to identify $\hat{\lambda}$ to a subset of $T^{1} S$, which is a union of orbits of the geodesic flow.

Locally choose a non-zero section $x \mapsto v_{a}(x)$ of the restriction of the line bundle $L_{a}$ over this subset $\hat{\lambda}$, in such a way that the section is flat over each of the leaves of $\hat{\lambda}$. This 
restriction is possible because the line bundle is flat over these leaves, by invariance of $L_{a}$ under the flat lift of the geodesic flow (see Theorem 3.1). The choice of the local section $x \mapsto v_{a}(x)$ locally defines a function $F_{a}$ on $\hat{\lambda}$ by the property that

$$
F_{a}(x)=-\log \left\|v_{a}(x)\right\|_{x}
$$

which is smooth along each leaf of $\hat{\lambda}$. Then $\omega_{a}$ is defined as $\omega_{a}=d F_{a}$ on each leaf of $\hat{\lambda}$. The construction is designed so that $\omega_{a}$ does not depend on the choice of the local section $v_{a}(x)$. However, its "antidifferential" $F_{a}$ does, and the crux of the argument is to choose the local sections $x \mapsto v_{a}(x)$ so that locally $F_{a}$ only changes by a constant as one moves from one chart to another, as required in the definition of topological closed 1-forms. We will use the slithering maps of $\S 5.1$ for this purpose.

Every $x \in \hat{\lambda}$ admits a small neighborhood $V_{x} \subset \widehat{U}$ such that, if we lift $V_{x}$ to an open subset $\widetilde{V}_{x}$ in the universal covering $\widetilde{S}$, the leaves of $\tilde{\lambda} \subset \widetilde{S}$ that meet $\widetilde{V}_{x}$ are parallel in the following sense: given any three such leaves $g, g^{\prime}$ and $g^{\prime \prime}$, one of them separates the other two in $\widetilde{S}$. The leaves of $\tilde{\lambda} \cap \widetilde{V}_{x}$ are oriented by the canonical orientation of the leaves of $\hat{\lambda} \cap V_{x}$. As in $\S 5.1$, the lift of the line bundle $L_{a}$ associates a line $\tilde{L}_{a}(g) \subset \mathbb{R}^{n}$ with each oriented leaf of $\tilde{\lambda}$.

Pick an arbitrary non-zero vector $\tilde{v}_{a}^{x}(x) \in \tilde{L}_{a}\left(g_{x}\right)$ for the leaf $g_{x}$ of $\tilde{\lambda}$ that contains the lift $\tilde{x} \in \widetilde{V}_{x}$ of $x \in V_{x}$. Then, for every $y \in V_{x} \cap \hat{\lambda}$, define $\tilde{v}_{a}^{x}(y)=\Sigma_{g_{y} g_{x}}\left(\tilde{v}_{a}^{x}(x)\right) \in \tilde{L}_{a}\left(g_{y}\right)$, where $\Sigma_{g_{y} g_{x}}: \mathbb{R}^{n} \rightarrow \mathbb{R}^{n}$ is the slithering map of $\S 5.1$. This map $\tilde{v}_{a}$ projects to a section $v_{a}$ of the line bundle $L_{a}$ over $V_{x} \cap \hat{\lambda}$, which is flat along the leaves of $V_{x} \cap \hat{\lambda}$ because $\tilde{v}_{a}^{x}(y)$ depends only on the leaf $g_{y}$, and which is Hölder continuous because of the Hölder continuity property of the slithering map $\Sigma_{g_{y} g_{x}}$ (condition (2) of Proposition 5.1). We can then consider the Hölder continuous function $F_{a}^{x}: V_{x} \cap \hat{\lambda} \rightarrow \mathbb{R}$ defined by

$$
F_{a}^{x}(y)=-\log \left\|v_{a}^{x}(y)\right\|_{y}
$$

We claim that the family $\left\{\left(V_{x} \cap \hat{\lambda}, F_{a}^{x}\right)\right\}_{x \in \hat{\lambda}}$ defines a Hölder continuous topological 1form $\Omega_{a}$. Namely, we will prove that $F_{a}^{x}-F_{a}^{x^{\prime}}$ is locally constant near each $y \in V_{x} \cap V_{x^{\prime}} \cap \hat{\lambda}$.

Consider such a point $y \in V_{x} \cap V_{x^{\prime}} \cap \hat{\lambda}$. We arranged that the leaves of $\tilde{\lambda}$ that meet $\widetilde{V}_{x}$ are parallel to each other. By Lemma 5.6, it follows that for every $z \in V_{x} \cap V_{x^{\prime}} \cap \hat{\lambda}$

$$
\tilde{v}_{a}^{x}(z)=\Sigma_{g_{z} g_{x}}\left(\tilde{v}_{a}^{x}(x)\right)=\Sigma_{g_{z} g_{y}} \circ \Sigma_{g_{y} g_{x}}\left(\tilde{v}_{a}^{x}(x)\right)=\Sigma_{g_{z} g_{y}}\left(\tilde{v}_{a}^{x}(y)\right)
$$

In the construction of the local sections $v_{a}^{x}$ and $v_{a}^{x^{\prime}}$, we can choose the lift $\widetilde{V}_{x^{\prime}} \subset \widetilde{S}$ so that it contains the lift $\tilde{y} \in \widetilde{V}_{x}$ of $y \in V_{x} \cap V_{x^{\prime}}$. In particular, the vectors $\tilde{v}_{a}^{x}(y)$ and $\tilde{v}_{a}^{x^{\prime}}(y)$ are both in the line $\tilde{L}_{a}\left(g_{y}\right)$ associated with the leaf of $\tilde{\lambda}$ passing through $\tilde{y}$, and there exists 


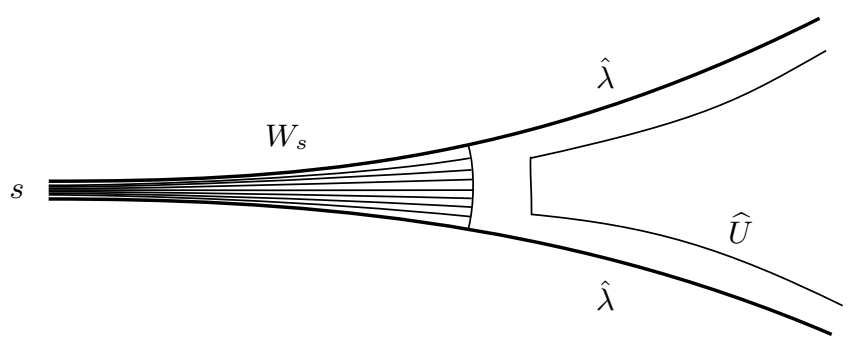

Figure 9. A spike $s$ of $\widehat{U} \backslash \hat{\lambda}$ and its neighborhood $W_{s}$.

a non-zero number $c \in \mathbb{R}$ such that $\tilde{v}_{a}^{x^{\prime}}(y)=c \tilde{v}_{a}^{x}(y)$. Each $z \in V_{x} \cap V_{x^{\prime}} \cap \hat{\lambda}$ that is sufficiently close to $y$ lifts to the same point $\tilde{z}$ in $\widetilde{V}_{x^{\prime}}$ and in $\widetilde{V}_{x}$, and as before,

$$
\tilde{v}_{a}^{x^{\prime}}(z)=\Sigma_{g_{z} g_{y}}\left(\tilde{v}_{a}^{x^{\prime}}(y)\right)=\Sigma_{g_{z} g_{y}}\left(c \tilde{v}_{a}^{x}(y)\right)=c \tilde{v}_{a}^{x}(z)
$$

It follows that $v_{a}^{x^{\prime}}(z)=c v_{a}^{x}(z)$ for $z \in \hat{\lambda}$ sufficiently close to $y$.

This proves that

$$
F_{a}^{x}(z)-F_{a}^{x^{\prime}}(z)=-\log \left\|v_{a}^{x}(z)\right\|_{z}+\log \left\|v_{a}^{x^{\prime}}(z)\right\|_{z}=-\log \left\|v_{a}^{x}(z)\right\|_{z}+\log \left\|c v_{a}^{x}(z)\right\|_{z}=\log |c|
$$

for every $z \in \hat{\lambda}$ near $y$. As a consequence, we have that $F_{a}^{x}-F_{a}^{x^{\prime}}$ is locally constant near each $y \in V_{x} \cap V_{x^{\prime}} \cap \hat{\lambda}$.

This concludes the proof that the family $\left\{\left(V_{x} \cap \hat{\lambda}, F_{a}^{x}\right)\right\}_{x \in \hat{\lambda}}$ defines a Hölder continuous topological closed 1-form $\Omega_{a}$ on $\hat{\lambda}$. This form $\Omega_{a}$ was specially designed so that its restriction to the leaves of $\hat{\lambda}$ coincides with the original 1 -form $\omega_{a}$.

We now extend $\Omega_{a}$ to the whole train-track neighborhood $\widehat{U}$.

LEMma 7.8. There exists a Hölder continuous topological closed 1-form $\Omega_{a}$ on the train-track neighborhood $\widehat{U}$ of $\hat{\lambda}$ whose restriction to each leaf of $\hat{\lambda}$ is equal to the form $\omega_{a}$.

Proof. Each spike $s$ of $\widehat{U} \backslash \hat{\lambda}$ admits a neighborhood $W_{s}$ foliated by half-geodesics converging to $s$; see Figure 9 . Choose these neighborhoods so that they are pairwise disjoint, and so that each of them is delimited by a differentiable (say) arc.

The union $W$ of $\hat{\lambda}$ and of these neighborhoods $W_{s}$ is then foliated by complete geodesics (namely leaves of $\hat{\lambda}$ ) and complete half-geodesics (in the neighborhoods $W_{s}$ of the spikes). The slithering maps of $\S 5.1$ straightforwardly extend to the preimage of $W$ in the universal cover $\widetilde{S}$, by elementary slitherings on each component of the preimage of the $W_{s}$. This enables us to use the argument of the proof of Lemma 7.7 to extend the topological closed 1-form $\Omega_{a}$ to $W$. 
Each component $A$ of $\widehat{U} \backslash W$ is an annulus, bounded on one side by a component of $\partial \widehat{U}$ and on the other side by a dodecagon, made up of six arcs contained in leaves of $\hat{\lambda}$ and of six arcs delimiting the $W_{s}$ associated with the six spikes of the component of $\widehat{U} \backslash \hat{\lambda}$ that contains $A$. As a consequence, there is a Hölder continuous retraction $r: \widehat{U} \rightarrow W$. We can then pull back by $r$ the topological closed 1-form $\Omega$ that we have constructed on $W$ to extend it to $\widehat{U}$.

Since the retraction $r$ fixes $W$, this does not change the restriction of $\Omega$ to the leaves of $\hat{\lambda}$, which consequently coincides with the form $\omega_{a}=f_{a} d t$.

By Lemma 7.6, the topological closed 1-form $\Omega_{a}$ provided by Lemma 7.8 defines a cohomology class $\left[\Omega_{a}\right] \in H^{1}(\widehat{U} ; \mathbb{R})$. Also, a tangent cycle $\alpha \in Z(\hat{\lambda} ; \mathbb{R})$ determines a homology class $[\alpha] \in H_{1}(\widehat{U} ; \mathbb{R})$ by Proposition 4.2 . The following statement connects these to the length $\ell_{a}^{\varrho}(\alpha) \in \mathbb{R}$.

LEMMA 7.9. For every tangent cycle $\alpha \in \mathcal{Z}(\hat{\lambda} ; \mathbb{R})$, the length $\ell_{a}^{\varrho}(\alpha)$ is equal to the evaluation

$$
\ell_{a}^{\varrho}(\alpha)=\left\langle\left[\Omega_{a}\right]-\left[\Omega_{a+1}\right],[\alpha]\right\rangle
$$

of the cohomology class $\left[\Omega_{a}\right]-\left[\Omega_{a+1}\right] \in H^{1}(\widehat{U} ; \mathbb{R})$ over the homology class $[\alpha] \in H_{1}(\widehat{U} ; \mathbb{R})$.

Proof. The tangent cycle $\alpha \in Z(\hat{\lambda} ; \mathbb{R})$ defines a transverse Hölder distribution for the geodesic lamination $\hat{\lambda}$; see [7] and [6]. As in [38], we can then interpret the data of the geodesic lamination $\hat{\lambda}$ endowed with this transverse Hölder distribution as a closed de Rham current in $\widehat{U}$. The homology class of $H_{1}(\widehat{U} ; \mathbb{R})$ defined by this de Rham current is exactly the class $[\alpha]$ introduced in Proposition 4.2 .

By definition, the length $\ell_{a}^{\varrho}(\alpha)$ is obtained by locally integrating the differential form $\omega_{a}-\omega_{a+1}$ over the leaves of $\hat{\lambda}$, and then integrating the corresponding function of the leaves of $\hat{\lambda}$ with respect to the transverse Hölder distribution defined by $\alpha$. See [15] for precise details, using a suitable partition of unity for $T^{1} S$. Since $\omega_{a}$ is the restriction of the topological closed 1-form $\Omega$ to the leaves of $\hat{\lambda}$, this construction is identical to the expression of [38] for the evaluation of $\left[\Omega_{a}-\Omega_{a+1}\right] \in H^{1}(\widehat{U} ; \mathbb{R})$ over the homology class $[\alpha] \in H_{1}(\widehat{U} ; \mathbb{R})$ represented by the de Rham current $\alpha \in \mathcal{C}^{\mathrm{H}}(\hat{\lambda})$.

\subsubsection{A homological interpretation of the shearing cycle $\sigma_{a}^{\varrho}$}

We now relate the shearing cycle $\sigma_{a}^{\varrho}$ to the topological closed 1-form $\Omega_{a}$ provided by Lemma 7.8 .

For this, consider an arc $k$ in $\widehat{U}$ that is tightly transverse to $\hat{\lambda}$. As usual, orient $k$ to the right of the leaves of $\hat{\lambda}$, and lift $k$ to an oriented arc $\tilde{k}$ in the universal cover $\widetilde{S}$. 


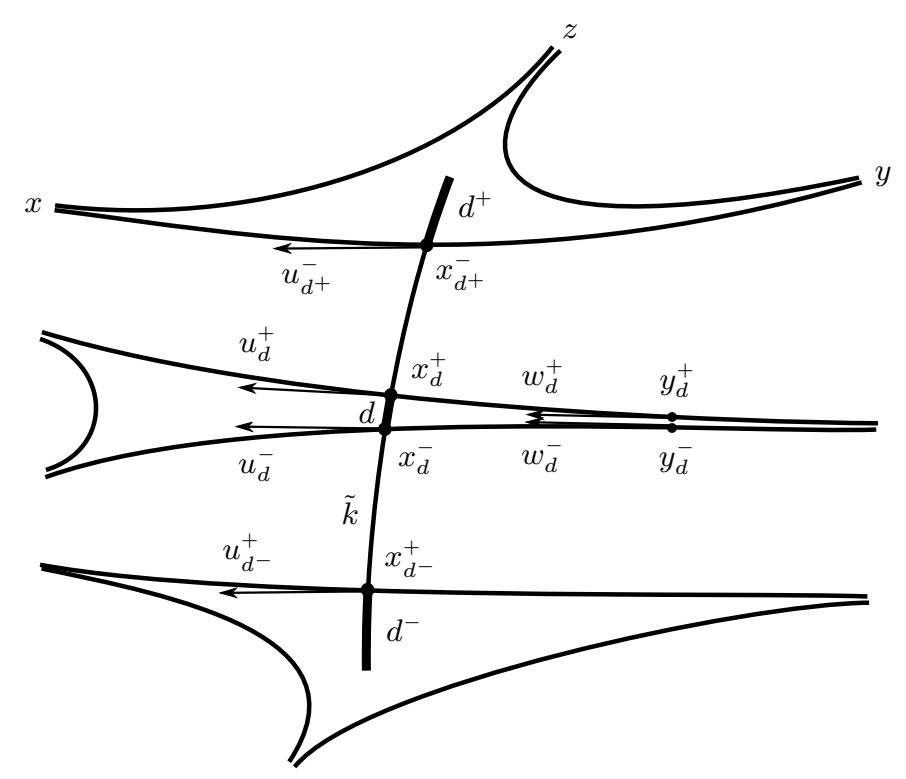

Figure 10. Integrating $\Omega_{a}$ over a tightly transverse $\operatorname{arc} k$.

Consistently with the canonical orientation of the leaves of $\hat{\lambda}$, we orient the leaves of $\tilde{\lambda}$ that meet $\tilde{k}$ to the left of $\tilde{k}$.

We first consider a component $d$ of $\tilde{k} \backslash \tilde{\lambda}$ that does not contain any of the two endpoints of $k$. In particular, the positive and negative endpoints $x_{d}^{+}$and $x_{d}^{-}$of $d$ belong to $\tilde{\lambda}$.

The tangent of the oriented leaf of $\tilde{\lambda}$ passing through $x_{d}^{ \pm}$determines an element $u_{d}^{ \pm} \in T^{1} \widetilde{S}$ of the unit tangent bundle of $\widetilde{S}$. If $g_{d}^{ \pm}$denotes the leaf of $\tilde{\lambda}$ passing through $x_{d}^{ \pm}$ and if we use the same letter to denote the projection $d \subset k \subset \widehat{U}$ of the arc $d \subset \tilde{k} \subset \widetilde{S}$, we now connect the integral $\int_{d} \Omega_{a}$ to the elementary slithering map $\Sigma_{g_{d}^{+} g_{d}^{-}}: \mathbb{R}^{n} \rightarrow \mathbb{R}^{n}$. The riemannian metric on the vector bundle $T^{1} S \widetilde{\times} \varrho_{\varrho^{\prime}} \mathbb{R}^{n}$ used in the definition of the forms $\Omega_{a}=f_{a} d t$ along $\hat{\lambda}$ defines, for each $u \in T^{1} \widetilde{S}$, a norm $\|\cdot\|_{u}$ on $\mathbb{R}^{n}$.

LEMMA 7.10. Let $k$ be an arc in $\widehat{U}$ that is tightly transverse to $\hat{\lambda}$, and let $d$ be a component of $k \backslash \hat{\lambda}$ that contains none of the two endpoints of $k$. Then,

$$
\int_{d} \Omega_{a}=\log \frac{\left\|v_{a}\left(u_{d}^{-}\right)\right\|_{u_{d}^{-}}}{\left\|\Sigma_{g_{d}^{+} g_{d}^{-}}\left(v_{a}\left(u_{d}^{-}\right)\right)\right\|_{u_{d}^{+}}}
$$

for any non-zero vector $v_{a}\left(u_{d}^{-}\right)$in the line $\tilde{L}_{a}\left(u_{d}^{-}\right)$.

The property is rather immediate when $\Omega_{a}$ is the form provided by our proof of Lemmas 7.7 and 7.8, and when $d$ is sufficiently small. However, we need a proof that is 
valid for all components $d$ of $k \backslash \hat{\lambda}$ that are disjoint from the endpoints. Our argument will be valid for all forms $\Omega_{a}$ satisfying the conclusions of Lemma 7.8, not just the one provided by our proof.

Proof. The two leaves $g_{d}^{+}$and $g_{d}^{-}$are asymptotic. We can therefore find points $y_{d}^{+} \in g_{d}^{+}$and $y_{d}^{-} \in g_{d}^{-}$which are arbitrarily close to each other. Let $w_{d}^{ \pm}$be the element of the unit tangent bundle $T^{1} \widetilde{S}$ determined by the tangent of the oriented geodesic $g_{d}^{ \pm}$at the point $y_{d}^{ \pm}$. See Figure 10. We can then deform $d$ to an arc consisting of the arc from $x_{d}^{-}$to $y_{d}^{-}$in the leaf $g_{d}^{-}$, followed by a short arc from $y_{d}^{-}$to $y_{d}^{+}$, and completed by the arc from $y_{d}^{+}$to $x_{d}^{+}$in $g_{d}^{+}$. Then, by definition of the restriction $\omega_{a}$ of $\Omega_{a}$ to the leaves of $\hat{\lambda}$,

$$
\int_{d} \Omega_{a}=\int_{x_{d}^{-}}^{y_{d}^{-}} \Omega_{a}+\int_{y_{d}^{-}}^{y_{d}^{+}} \Omega_{a}+\int_{y_{d}^{+}}^{x_{d}^{+}} \Omega_{a}=\log \frac{\left\|v_{a}\left(u_{d}^{-}\right)\right\|_{u_{d}^{-}}}{\left\|v_{a}\left(u_{d}^{-}\right)\right\|_{w_{d}^{-}}}+\int_{y_{d}^{-}}^{y_{d}^{+}} \Omega_{a}+\log \frac{\left\|v_{a}\left(u_{d}^{+}\right)\right\|_{w_{d}^{+}}}{\left\|v_{a}\left(u_{d}^{+}\right)\right\|_{u_{d}^{+}}}
$$

for arbitrary non-zero vectors $v_{a}\left(u_{d}^{+}\right) \in \tilde{L}_{a}\left(u_{d}^{+}\right)$and $v_{a}\left(u_{d}^{-}\right) \in \tilde{L}_{a}\left(u_{d}^{-}\right)$. In particular, we can choose $v_{a}\left(u_{d}^{+}\right)=\Sigma_{g_{d}^{+} g_{d}^{-}}\left(v_{a}\left(u_{d}^{-}\right)\right)$, in which case

$$
\int_{d} \Omega_{a}=\log \frac{\left\|v_{a}\left(u_{d}^{-}\right)\right\|_{u_{d}^{-}}}{\left\|\Sigma_{g_{d}^{+} g_{d}^{-}}\left(v_{a}\left(u_{d}^{-}\right)\right)\right\|_{u_{d}^{+}}}-\log \frac{\left\|v_{a}\left(u_{d}^{-}\right)\right\|_{w_{d}^{-}}}{\left\|\Sigma_{g_{d}^{+} g_{d}^{-}}\left(v_{a}\left(u_{d}^{-}\right)\right)\right\|_{w_{d}^{+}}}+\int_{y_{d}^{-}}^{y_{d}^{+}} \Omega_{a} .
$$

Now, we let the points $y_{d}^{+}$and $y_{d}^{-}$tend to the common endpoint of $g_{d}^{+}$and $g_{d}^{-}$in such a way that the distance from $y_{d}^{+}$to $y_{d}^{-}$tends to zero. Looking at the projections to $S$, the integral $\int_{y_{d}^{-}}^{y^{+}} \Omega_{a}$ tends to zero by continuity of the functions $F_{a}$ that locally define $\Omega_{a}$, while the quotient

$$
\frac{\left\|v_{a}\left(u_{d}^{-}\right)\right\|_{w_{d}^{-}}}{\left\|\Sigma_{g_{d}^{+} g_{d}^{-}}\left(v_{a}\left(u_{d}^{-}\right)\right)\right\|_{w_{d}^{+}}}
$$

tends to 1 (compare Lemma 5.2 and use the $\varrho$-equivariance of the riemannian metric $\|\cdot\|)$. It follows that

$$
\int_{d} \Omega_{a}=\log \frac{\left\|v_{a}\left(u_{d}^{-}\right)\right\|_{u_{d}^{-}}}{\left\|\Sigma_{g_{d}^{+} g_{d}^{-}}\left(v_{a}\left(u_{d}^{-}\right)\right)\right\|_{u_{d}^{+}}}
$$

for any non-zero vector $v_{a}\left(u_{d}^{-}\right) \in \tilde{L}_{a}\left(u_{d}^{-}\right)$.

We will now choose preferred vectors $v_{a}\left(u_{d}^{-}\right) \in \tilde{L}_{a}\left(u_{d}^{-}\right)$.

Let $d^{+}$and $d^{-}$be the components of $\tilde{k} \backslash \tilde{\lambda}$ that contain the positive and negative endpoints of $\tilde{k}$, respectively. In particular, their endpoints $x_{d^{+}}^{-}$and $x_{d^{-}}^{+}$are the points of $\tilde{k} \cap \tilde{\lambda}$ that are closest to the positive and negative endpoints in $\tilde{k}$, respectively. As usual, let $u_{d^{ \pm}}^{\mp} \in T^{1} \widetilde{S}$ be defined by the vector tangent to the (oriented) leaf $g_{d^{ \pm}}^{\mp}$ of $\tilde{\lambda}$ passing through $x_{d^{ \pm}}^{\mp}$. See Figure 10. 
The flag map $\mathcal{F}_{\varrho}: \partial_{\infty} \widetilde{S} \rightarrow \operatorname{Flag}\left(\mathbb{R}^{n}\right)$ associates several lines of $\mathbb{R}^{n}$ with the vector $u_{d^{+}}^{-} \in T^{1} \widetilde{S}$. This includes the $n$ lines $\tilde{L}_{a}\left(u_{d^{+}}^{-}\right)=\mathcal{F}_{\varrho}(x)^{(a)} \cap \mathcal{F}_{\varrho}(y)^{(n-a+1)}$ of $\S 3.1$, defined by the flags $\mathcal{F}_{\varrho}(x)$ and $\mathcal{F}_{\varrho}(y)$ respectively associated with the positive endpoint $x$ and the negative endpoint $y$ of the leaf $g_{d^{+}}^{-}$. We can also consider the line $\mathcal{F}_{\varrho}(z)^{(1)}$ of the flag $\mathcal{F}_{\varrho}(z)$ associated with the third vertex $z$ of the triangle component of $\widetilde{S} \backslash \tilde{\lambda}$ that contains $d^{+}$. Pick a non-trivial vector $v\left(u_{d^{+}}^{-}\right)$in this line $\mathcal{F}_{\varrho}(z)^{(1)}$, and let $v_{a}\left(u_{d^{+}}^{-}\right) \in \tilde{L}_{a}\left(u_{d^{+}}^{-}\right)$be the projection of $v\left(u_{d^{+}}^{-}\right)$parallel to the $\tilde{L}_{b}\left(u_{d^{+}}^{-}\right)$with $b \neq a$.

In particular, the quantity

$$
\frac{\left\|v_{a}\left(u_{d^{+}}^{-}\right)\right\|_{u_{d^{+}}^{-}}}{\left\|v_{a+1}\left(u_{d^{+}}^{-}\right)\right\|_{u_{d^{+}}^{-}}}
$$

is independent of the choice of the vector $v\left(u_{d^{+}}^{-}\right) \in \mathcal{F}_{\varrho}(z)^{(1)}$. Note that this ratio is finite and positive by genericity of the flag triple $\left(\mathcal{F}_{\varrho}(x), \mathcal{F}_{\varrho}(y), \mathcal{F}_{\varrho}(z)\right)$.

We can introduce similar definitions at the point $x_{d^{-}}^{+}$of $\tilde{k} \cap \tilde{\lambda}$ that is closest to the negative endpoint of $\tilde{k}$. Considering the triangle component of $\widetilde{S} \backslash \tilde{\lambda}$ that contains the negative endpoint of $\tilde{k}$, this leads to a well-defined positive ratio

$$
\frac{\left\|v_{a}\left(u_{d^{-}}^{+}\right)\right\|_{u_{d^{-}}^{+}}}{\left\|v_{a+1}\left(u_{d^{-}}^{+}\right)\right\|_{u_{d^{-}}^{+}}}
$$

LEMMA 7.11. Let $k$ be an arc in $\widehat{U}$ that is tightly transverse to $\hat{\lambda}$. Then, for the above definitions,

$$
\sigma_{a}^{\varrho}(k)=\int_{k \backslash\left(d^{+} \cup d^{-}\right)}\left(\Omega_{a}-\Omega_{a+1}\right)+\log \frac{\left\|v_{a}\left(u_{d^{+}}^{-}\right)\right\|_{u_{d^{+}}^{-}}}{\left\|v_{a+1}\left(u_{d^{+}}^{-}\right)\right\|_{u_{d^{+}}^{-}}} \log \frac{\left\|v_{a}\left(u_{d^{-}}^{+}\right)\right\|_{u_{d^{+}}^{+}}}{\left\|v_{a+1}\left(u_{d^{-}}^{+}\right)\right\|_{u_{d^{+}}^{+}}} .
$$

Note that the notation is ambiguous in the special case where $u_{d^{+}}^{-}=u_{d^{-}}^{+}$, which occurs when the arc $k$ crosses $\hat{\lambda}$ in only one point. We will leave to the reader the easy task of lifting the ambiguity in this case.

Proof. By a well-known result of Birman-Series [1], the intersection $\tilde{k} \cap \tilde{\lambda}$ has Hausdorff dimension zero. Since the topological closed 1-form $\Omega_{a}$ is Hölder continuous, if follows that

$$
\int_{k \backslash\left(d^{+} \cup d^{-}\right)} \Omega_{a}=\sum_{d} \int_{d} \Omega_{a}
$$

where the sum is over all components $d$ of $\tilde{k} \backslash \tilde{\lambda}$ that are different from $d^{+}$and $d^{-}$. (The critical property is that the image of a set of Hausdorff dimension zero under a Hölder continuous function has Hausdorff dimension zero, and in particular has Lebesgue measure zero in $\mathbb{R}$.) 
We now apply Lemma 7.10 while choosing $v_{a}\left(u_{d}^{-}\right)=\Sigma_{g_{d}^{-}} g_{d^{-}}\left(v_{a}\left(u_{d^{-}}^{+}\right)\right) \in L_{a}\left(u_{d}^{-}\right)$, where $v_{a}\left(u_{d^{-}}^{+}\right) \in \tilde{L}_{a}\left(u_{d^{-}}^{+}\right)$is determined as above by the vertices of the triangle component of $\widetilde{S} \backslash \tilde{\lambda}$ that contains $d^{+}$. Then,

$$
\int_{k \backslash\left(d^{+} \cup d^{-}\right)} \Omega_{a}=\sum_{d} \log \frac{\left\|\Sigma_{g_{d}^{-} g_{d^{-}}^{+}}\left(v_{a}\left(u_{d^{-}}^{+}\right)\right)\right\|_{u_{d}^{-}}}{\left\|\Sigma_{g_{d}^{+} g_{d^{-}}^{+}}\left(v_{a}\left(u_{d^{-}}^{+}\right)\right)\right\|_{u_{d}^{+}}}
$$

by observing that

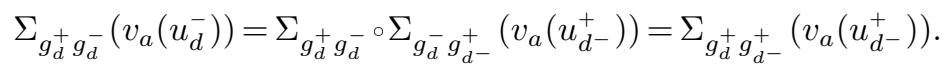

If $g_{x}$ denotes the oriented leaf of $\tilde{\lambda}$ passing through $x \in \tilde{k} \cap \tilde{\lambda}$ and if $u_{x} \in T^{1} \widetilde{S}$ is the unit vector tangent to $g_{x}$ at $x$, the map $x \mapsto\left\|\Sigma_{g_{x} g_{d^{+}}}\left(v_{a}\left(u_{d^{-}}^{+}\right)\right)\right\|_{u_{x}}$ is Hölder continuous, because $g_{x}$ depends Lipschitz continuously on $x$ by [13, $\left.\S 5.2 .6\right]$, and because the slithering map $\Sigma_{g g_{d^{-}}}$is a Hölder continuous function of the leaf $g$ by Proposition 5.1. Using again the fact that $\tilde{k} \cap \tilde{\lambda}$ has Hausdorff dimension zero, it follows that

$$
\int_{k \backslash\left(d^{+} \cup d^{-}\right)} \Omega_{a}=\sum_{d} \log \frac{\left\|\Sigma_{g_{d}^{-} g_{d^{-}}^{+}}\left(v_{a}\left(u_{d^{-}}^{+}\right)\right)\right\|_{u_{d}^{-}}}{\left\|\Sigma_{g_{d}^{+} g_{d^{-}}^{+}}\left(v_{a}\left(u_{d^{-}}^{+}\right)\right)\right\|_{u_{d}^{+}}}=\log \frac{\left\|v_{a}\left(u_{d^{-}}^{+}\right)\right\|_{u_{d^{-}}^{+}}}{\left\|\Sigma_{g_{d^{+}} g_{d^{-}}^{+}}\left(v_{a}\left(u_{d^{-}}^{+}\right)\right)\right\|_{u_{d^{+}}^{-}}} .
$$

By construction, the slithering map $\Sigma_{g_{d^{-}}^{-} g_{d^{-}}^{+}}$sends $\tilde{L}_{a}\left(u_{d^{-}}^{+}\right)$to $\tilde{L}_{a}\left(u_{d^{+}}^{-}\right)$. In particular, there exists a non-zero number $\mu_{a}$ such that $\Sigma_{g_{d^{+}}^{-} g_{d^{-}}^{+}}\left(v_{a}\left(u_{d^{-}}^{+}\right)\right)=\mu_{a} v_{a}\left(u_{d^{+}}^{-}\right)$. Then,

$$
\begin{aligned}
\int_{k \backslash\left(d^{+} \cup d^{-}\right)}\left(\Omega_{a}-\Omega_{a+1}\right) & =\log \frac{\left\|v_{a}\left(u_{d^{-}}^{+}\right)\right\|_{u_{d^{-}}^{+}}}{\left\|v_{a+1}\left(u_{d^{-}}^{+}\right)\right\|_{u_{d^{+}}^{+}}}-\log \frac{\left\|v_{a}\left(u_{d^{+}}^{-}\right)\right\|_{u_{d^{+}}^{-}}}{\left\|v_{a+1}\left(u_{d^{+}}^{-}\right)\right\|_{u_{d^{+}}^{-}}}-\log \left|\frac{\mu_{a}}{\mu_{a+1}}\right| \\
& =\log \frac{\left\|v_{a}\left(u_{d^{-}}^{+}\right)\right\|_{u_{d^{-}}^{+}}}{\left\|v_{a+1}\left(u_{d^{-}}^{+}\right)\right\|_{u_{d^{-}}^{+}}}-\log \frac{\left\|v_{a}\left(u_{d^{+}}^{-}\right)\right\|_{u_{d^{+}}^{-}}}{\left\|v_{a+1}\left(u_{d^{+}}^{-}\right)\right\|_{u_{d^{+}}^{-}}}+\sigma_{a}^{\varrho}(k),
\end{aligned}
$$

by definition of the shearing parameter $\sigma_{a}^{\varrho}(k)$ in $\S 5.2$ (and using Lemma 1.6).

Lemma 7.12. For every homology class $[\alpha] \in H_{1}(\widehat{U} ; \mathbb{R})$,

$$
\left\langle\left[\Omega_{a}\right]-\left[\Omega_{a+1}\right],[\alpha]\right\rangle=[\alpha] \cdot\left[\sigma_{a}^{\varrho}\right] .
$$

Proof. We already observed, in the proof of Proposition 4.5, that $H_{1}\left(\widehat{U}, \partial_{\mathrm{h}} \widehat{U} ; \mathbb{R}\right)$ admits a basis where each element is represented by a generic tie of $\widehat{U}$. We can therefore write the image $[\alpha] \in H_{1}\left(\widehat{U}, \partial_{\mathrm{h}} \widehat{U} ; \mathbb{R}\right)$ as a linear combination $[\alpha]=\sum_{i} \mu_{i}\left[k_{i}\right]$ of classes represented by generic ties $k_{i}$, with coefficients $\mu_{i} \in \mathbb{R}$.

Recall that the ties of $\widehat{U}$ are oriented to the right for the canonical orientation of the leaves of $\hat{\lambda}$. In particular, the components of the horizontal boundary $\partial_{\mathrm{h}} \widehat{U}$ are of two 
types: those components where the orientation of the ties point outside of $\widehat{U}$, and those where it points inside. Also, because of this orientation convention,

$$
[\alpha] \cdot\left[\sigma_{a}^{\varrho}\right]=\sum_{i} \mu_{i}\left[k_{i}\right] \cdot\left[\sigma_{a}^{\varrho}\right]=\sum_{i} \mu_{i} \sigma_{a}^{\varrho}\left(k_{i}\right)
$$

by definition of the homology class $\left[\sigma_{a}^{\varrho}\right] \in H_{1}\left(\widehat{U}, \partial_{\mathrm{v}} \widehat{U} ; \mathbb{R}\right)$ associated with the relative tangent cycle $\sigma_{a}^{\varrho} \in Z(\hat{\lambda}$, slits; $\mathbb{R})$ by Proposition 4.5 .

We now modify each arc $k_{i}$ by a homotopy respecting $\hat{\lambda}$ and $\partial_{\mathrm{h}} \widehat{U}$ to obtain an arc $k_{i}^{\prime}$ such that the following holds: for every component $C$ of the horizontal boundary $\partial_{\mathrm{h}} \widehat{U}$, there is an arc $k_{C} \subset \widehat{U}$ such that, for every arc $k_{i}^{\prime}$ with an endpoint in $C$, the component of $k_{i}^{\prime} \backslash \hat{\lambda}$ containing this endpoint is equal to $k_{C}$. The only case where this regrouping of arcs near the horizontal boundary requires some care is when the original tie $k_{i}$ meets $\hat{\lambda}$ in one point; in this special situation, one needs to first choose the relevant arcs $k_{C}$ so that $k_{i}^{\prime}=k_{i}$, and then modify the other $k_{j}$ accordingly.

Now, by Lemma 7.11,

$$
\begin{gathered}
\sum_{i} \mu_{i} \sigma_{a}^{\varrho}\left(k_{i}^{\prime}\right)=\sum_{i} \mu_{i} \int_{k_{i}^{\prime}}\left(\Omega_{a}-\Omega_{a+1}\right)-\sum_{i} \mu_{i} \int_{d_{i}^{+}}\left(\Omega_{a}-\Omega_{a+1}\right)-\sum_{i} \mu_{i} \int_{d_{i}^{-}}\left(\Omega_{a}-\Omega_{a+1}\right) \\
+\sum_{i} \mu_{i} \log \frac{\left\|v_{a}\left(u_{d_{i}^{+}}^{-}\right)\right\|_{u_{d_{i}^{+}}^{-}}}{\left\|v_{a+1}\left(u_{d_{i}^{-}}^{-}\right)\right\|_{u_{d_{i}^{+}}^{-}}}-\sum_{i} \mu_{i} \log \frac{\left\|v_{a}\left(u_{d_{i}^{+}}^{+}\right)\right\|_{u_{d_{i}^{+}}^{+}}}{\left\|v_{a+1}\left(u_{d_{i}^{+}}^{+}\right)\right\|_{u_{d_{i}^{+}}}}
\end{gathered}
$$

where $d_{i}^{+}$and $d_{i}^{-}$are the components of $k_{i}^{\prime} \backslash \hat{\lambda}$ containing the positive and negative endpoints of $k_{i}^{\prime}$, respectively. In particular, each $d_{i}^{ \pm}$is equal to one of the arcs $k_{C}$ associated with the components $C$ of the horizontal boundary $\partial_{\mathrm{h}} \widehat{U}$.

The key observation is now that $[\alpha]=\sum_{i} \mu_{i}\left[k_{i}\right] \in H_{1}\left(\widehat{U}, \partial_{\mathrm{h}} \widehat{U} ; \mathbb{R}\right)$ comes from an element of $H_{1}(\widehat{U} ; \mathbb{R})$, and in particular has boundary zero. This implies that, for each component $C$ of $\partial_{\mathrm{h}} \widehat{U}$ where the ties point outwards, the sum of the $\mu_{i}$ such that $k_{i}$ has an endpoint in $C$ is equal to zero; equivalently, the $\mu_{i}$ such that $d_{i}^{+}=k_{C}$ add up to zero. Similarly, for each component $C$ of $\partial_{\mathrm{h}} \widehat{U}$ where the ties point inwards, the sum of the coefficients $\mu_{i}$ such that $d_{i}^{-}=k_{C}$ is equal to zero.

This implies that most terms cancel out in the above sum, and that

$$
\sum_{i} \mu_{i} \sigma_{a}^{\varrho}\left(k_{i}^{\prime}\right)=\sum_{i} \mu_{i} \int_{k_{i}^{\prime}}\left(\Omega_{a}-\Omega_{a+1}\right)=\left\langle\left[\Omega_{a}\right]-\left[\Omega_{a+1}\right],[\alpha]\right\rangle .
$$

For the second equality note that, because the $\mu_{i}$ for which the positive (resp. negative) endpoint of $k_{i}^{\prime}$ is in a given component $C$ of $\partial_{\mathrm{h}} \widehat{U}$ add up to zero, the chain $\sum_{i} \mu_{i} k_{i}^{\prime}$ is closed and represents the class $[\alpha] \in H_{1}(\widehat{U} ; \mathbb{R})$. 
This proves that

$$
[\alpha] \cdot\left[\sigma_{a}\right]=\sum_{i} \mu_{i} \sigma_{a}\left(k_{i}\right)=\sum_{i} \mu_{i} \sigma_{a}\left(k_{i}^{\prime}\right)=\left\langle\left[\Omega_{a}\right]-\left[\Omega_{a+1}\right],[\alpha]\right\rangle .
$$

The combination of Lemmas 7.9 and 7.12 completes the proof of Theorem 7.5.

Corollary 7.13. Let $\mu$ be a non-trivial transverse measure for the orientation cover $\hat{\lambda}$, and let $[\mu] \in H_{1}(\widehat{U} ; \mathbb{R})$ be its associated homology class as in $\S 4.3$. Then,

$$
[\mu] \cdot\left[\sigma_{a}^{\varrho}\right]>0
$$

for each component $\sigma_{a}^{\varrho} \in \mathcal{Z}(\hat{\lambda}$, slits; $\mathbb{R}) \cong H_{1}\left(\widehat{U}, \partial_{\mathrm{v}} \widehat{U} ; \mathbb{R}\right)$ of the shearing cycle

$$
\sigma^{\varrho} \in Z\left(\lambda, \text { slits; } \widehat{\mathbb{R}}^{n-1}\right)
$$

of a Hichin character $\varrho \in \operatorname{Hit}_{n}(S)$.

Proof. This is an immediate consequence of Theorem 7.5 and Proposition 7.4.

\section{Parametrizing Hitchin components}

In $\S 3$ and $\S 5.2$, we associated certain invariants with a Hitchin character $\varrho \in \operatorname{Hit}_{n}(S)$.

The first type of invariants are the triangle invariants $\tau_{a b c}^{\varrho}(s)$, defined as $s$ ranges over the slits of $\lambda$ and as $a, b, c \geqslant 1$ range over all integers such that $a+b+c=n$. Noting that there are $\frac{1}{2}(n-1)(n-2)$ such triples $(a, b, c)$ and $12(g-1)$ slits of $S \backslash \lambda$, we can combine all these invariants into a single map

$$
\operatorname{Hit}_{n}(S) \longrightarrow \mathbb{R}^{6(g-1)(n-1)(n-2)} .
$$

The second invariant is the shearing cycle $\sigma^{\varrho} \in \mathcal{C}\left(\lambda\right.$, slits; $\left.\widehat{\mathbb{R}}^{n}\right)$, which provides a map

$$
\operatorname{Hit}_{n}(S) \longrightarrow \mathcal{C}\left(\lambda, \text { slits; } \widehat{\mathbb{R}}^{n}\right) \cong \mathbb{R}^{18(g-1)(n-1)},
$$

where the isomorphism is given by Proposition 4.7.

Combining these two maps, we define

$$
\Phi: \operatorname{Hit}_{n}(S) \longrightarrow \mathbb{R}^{6(g-1)(n-1)(n-2)} \times \mathfrak{C}\left(\lambda, \text { slits; } \widehat{\mathbb{R}}^{n}\right) \cong \mathbb{R}^{6(g-1)(n+1)(n-1)},
$$

which sends each Hitchin character $\varrho \in \operatorname{Hit}_{n}(S)$ to its triangle invariants and its shearing cycle. We proved in Corollary 6.8 that this map $\Phi$ is injective. We will show that $\Phi$ induces a homeomorphism between $\operatorname{Hit}_{n}(S)$ and an open convex polyhedral cone $\mathcal{P}$ contained in a linear subspace of $\mathbb{R}^{6(g-1)(n+1)(n-1)}$. 
LEMMA 8.1. The above map

$$
\Phi: \operatorname{Hit}_{n}(S) \longrightarrow \mathbb{R}^{6(g-1)(n-1)(n-2)} \times \mathcal{C}\left(\lambda, \text { slits } ; \widehat{\mathbb{R}}^{n}\right)
$$

is continuous.

Proof. The key property is that the flag curve $\mathcal{F}_{\varrho}: \partial_{\infty} \widetilde{S} \rightarrow$ Flag $\left(\mathbb{R}^{n}\right)$ depends continuously on the Hitchin homomorphism $\varrho: \pi_{1}(S) \rightarrow \mathrm{PSL}_{n}(\mathbb{R})$, and is uniformly Hölder continuous as $\varrho$ ranges over a compact subset of the space of homomorphisms $\pi_{1}(S) \rightarrow \mathrm{PSL}_{n}(\mathbb{R})$. These two properties follow from the application to the setup of $\S 3.1$ of the classical structural stability theorems for Anosov flows, and Hölder continuity properties for their stable and unstable foliations; see for instance [24, §18 and $\S 19]$.

The continuity property immediately shows that the triangle invariants $\tau_{a b c}^{\varrho}(s)$ depend continuously on $\varrho$.

The case of the shearing cycle $\sigma^{\varrho} \in \mathcal{C}\left(\lambda\right.$, slits; $\left.\widehat{\mathbb{R}}^{n}\right)$ requires an additional argument, because its construction relies on the slithering maps $\Sigma_{g g^{\prime}}: \mathbb{R}^{n} \rightarrow \mathbb{R}^{n}$. The uniform Hölder continuity property makes the estimates used in the construction of slithering maps in $\S 5.1$ uniform, and guarantees uniform convergence in this construction. It follows that, for any two leaves $g$ and $g^{\prime}$ of $\tilde{\lambda}$, the slithering map $\Sigma_{g g^{\prime}}$ depends continuously on $\varrho$. After this, the continuous dependence of the flag map $\mathcal{F}_{\varrho}$ on $\varrho$ is enough to prove that $\sigma^{\varrho}$ depends continuously on $\varrho$.

\subsection{Constraints between invariants}

There are clear constraints on the image of $\Phi$. The first one is the following consequence of Lemma 1.1, which we have already encountered in Lemma 3.4.

Triangle rotation condition. If the spikes of the component $T$ of $S \backslash \lambda$ are indexed as $s, s^{\prime}$ and $s^{\prime \prime}$ in counterclockwise order around $T$, then

$$
\tau_{a b c}^{\varrho}(s)=\tau_{b c a}^{\varrho}\left(s^{\prime}\right)=\tau_{c a b}^{\varrho}\left(s^{\prime \prime}\right) .
$$

The second constraint comes from the quasi-additivity property of the shearing cycle $\sigma^{\varrho}$. Recall that the lack of additivity of the ath component $\sigma_{a}^{\varrho} \in \mathcal{Z}\left(\hat{\lambda}\right.$, slits; $\left.\mathbb{R}^{n-1}\right)$ of

$\sigma^{\varrho} \in Z\left(\lambda\right.$, slits; $\left.\widehat{\mathbb{R}}^{n-1}\right) \subset \mathcal{Z}\left(\hat{\lambda}\right.$, slits; $\left.\mathbb{R}^{n-1}\right)$ is measured by its boundary $\partial \sigma_{a}^{\varrho}$, which associates a number $\partial \sigma_{a}^{\varrho}(\hat{s}) \in \mathbb{R}$ with each spike $\hat{s}$ of the orientation cover $\hat{\lambda}$ of the geodesic lamination $\lambda$. The spikes $\hat{s}$ can be positive or negative, according to whether the canonical orientation of the leaves of $\hat{\lambda}$ orients the two leaves that are adjacent to $\hat{s}$ towards $\hat{s}$ or away from $\hat{s}$ (namely, according to whether $\hat{s}$ sits at the positive or negative end of these leaves). 
The following constraint comes from the computation of $\partial \sigma_{a}^{\varrho}$ provided by Proposition 5.15 and Lemma 5.14 .

Shearing cycle boundary condition. For every positive slit $s^{+}$of $\hat{\lambda}$ projecting to a slit $s$ of $\lambda$,

$$
\partial \sigma_{a}^{\varrho}\left(s^{+}\right)=\sum_{b+c=n-a} \tau_{a b c}^{\varrho}(s) .
$$

Note that this property for positive slits, combined with the equivariance property of $\sigma^{\varrho} \in Z\left(\lambda\right.$, slits; $\left.\widehat{\mathbb{R}}^{n-1}\right) \subset Z\left(\hat{\lambda}\right.$, slits; $\left.\mathbb{R}^{n-1}\right)$ with respect to the covering involution of the cover $\hat{\lambda} \rightarrow \lambda$, determines $\partial \sigma_{a}^{\varrho}$ on negative slits. More precisely,

$$
\partial \sigma_{a}^{\varrho}\left(s^{-}\right)=-\sum_{b+c=a} \tau_{(n-a) b c}^{\varrho}(s)
$$

for every negative slit $s^{-}$of $\hat{\lambda}$ projecting to a slit $s$ of $\lambda$.

The last condition is provided by Corollary 7.13.

Positive intersection condition.

$$
[\mu] \cdot\left[\sigma_{a}^{\varrho}\right]>0
$$

for every non-trivial transverse measure $\mu$ for $\hat{\lambda}$, where

$$
[\mu] \in H_{1}(\widehat{U} ; \mathbb{R}) \quad \text { and } \quad\left[\sigma_{a}^{\varrho}\right] \in H_{1}\left(\widehat{U}, \partial_{\mathrm{v}} \widehat{U} ; \mathbb{R}\right)
$$

are the homology classes respectively defined by $\mu \in \mathcal{Z}(\hat{\lambda} ; \mathbb{R})$ and by the ath component $\sigma_{a}^{\varrho} \in \mathcal{Z}(\hat{\lambda}$, slits; $\mathbb{R})$ of the shearing cycle $\sigma^{\varrho} \in \mathcal{Z}\left(\lambda ; \widehat{\mathbb{R}}^{n-1}\right) \subset \mathcal{Z}\left(\hat{\lambda}\right.$, slits; $\left.\mathbb{R}^{n-1}\right)$, and where $\cdot$ denotes the algebraic intersection in $\widehat{U}$.

Let $\mathcal{P}$ be the set of pairs $(\tau, \sigma)$ such that

(1) $\tau$ is a function which associates a number $\tau_{a b c}(s) \in \mathbb{R}$ with each triple of integers $a, b, c \geqslant 1$ with $a+b+c=n$, and with each slit $s$ of $\lambda$;

(2) $\sigma \in \mathcal{Z}\left(\lambda\right.$, slits; $\left.\widehat{\mathbb{R}}^{n-1}\right)$ is a tangent cycle for $\lambda$ valued in the coefficient bundle $\widehat{\mathbb{R}}^{n-1}$ and relative to the slits of $\lambda$; in particular, $\sigma$ is defined by $n-1$ relative tangent cycles $\sigma_{a} \in \mathcal{Z}(\hat{\lambda}$, slits; $\mathbb{R}) ;$

(3) $\tau$ and $\sigma$ satisfy the above triangle rotation condition, shearing cycle boundary condition and positive intersection condition.

We will call a function $\tau \in \mathbb{R}^{6(g-1)(n-1)(n-2)}$ as in (1) a triangle data function. It is rotation-invariant when it satisfies the triangle rotation condition.

Proposition 8.2. The space $\mathcal{P}$ is an open convex polyhedral cone in a linear subspace of $\mathbb{R}^{6(g-1)(n-1)(n-2)} \times \mathcal{Z}\left(\lambda\right.$, slits; $\left.\widehat{\mathbb{R}}^{n-1}\right)$ of dimension $2(g-1)\left(n^{2}-1\right)$. 
Proof. The transverse measures for the geodesic lamination $\hat{\lambda}$ form a positive cone over a finite-dimensional simplex [25], [35]. It therefore suffices to check the positive intersection condition on the vertices of this simplex (corresponding to ergodic measures). This reduces the positive intersection condition to finitely many linear inequalities, in fact at most $6(g-1)$ such inequalities by the main result of [35]. As a consequence, $\mathcal{P}$ is an open convex polyhedral cone in the linear subspace of $\mathbb{R}^{6(g-1)(n-1)(n-2)} \times \mathfrak{C}\left(\lambda\right.$, slits; $\left.\widehat{\mathbb{R}}^{n}\right)$ defined by the triangle rotation condition and the shearing cycle boundary condition. We need to compute its dimension, which will require a few lemmas.

The triangle rotation condition divides the dimension of the space of triangle data functions by 3 , in the sense that the space of rotation-invariant triangle data functions $\tau \in \mathbb{R}^{6(g-1)(n-1)(n-2)}$ is isomorphic to $\mathbb{R}^{2(g-1)(n-1)(n-2)}$. Indeed, if we pick a spike $s_{j}$ for each triangle component $T_{j}$ of $S \backslash \lambda$, such a rotation-invariant $\tau$ is completely determined by the $2(g-1)(n-1)(n-2)$ numbers $\tau_{a b c}\left(s_{j}\right)$. We will use this observation to denote by $\mathbb{R}^{2(g-1)(n-1)(n-2)}$ the space of all rotation-invariant triangle data functions $\tau$.

Consider the linear subspace $\mathcal{L} \subset \mathbb{R}^{2(g-1)(n-1)(n-2)} \times \mathcal{Z}\left(\lambda\right.$, slits; $\left.\widehat{\mathbb{R}}^{n-1}\right)$ consisting of all pairs $(\tau, \sigma)$, where $\tau$ is a rotation-invariant triangle data function and $\sigma$ is a twisted tangent cycle for $\lambda$ relative to its slits, and where $\tau$ and $\sigma$ satisfy the shearing cycle boundary condition.

To analyze $\mathcal{L}$, we introduce a new vector space $\mathcal{Z}\left(\right.$ slits; $\left.\mathbb{R}^{n-1}\right)$, consisting of all functions $\xi:\{$ slits of $\lambda\} \rightarrow \mathbb{R}^{n-1}$. For $a=1,2, \ldots, n-1$, we denote the $a$ th component of such a $\xi \in \mathcal{Z}\left(\right.$ slits; $\left.\mathbb{R}^{n-1}\right)$ by $\xi_{a}:\{$ slits of $\lambda\} \rightarrow \mathbb{R}$. The definition of the space $\mathcal{L}$ can then be expressed in terms of two maps

$$
\partial: \mathcal{Z}\left(\lambda, \text { slits; } \widehat{\mathbb{R}}^{n-1}\right) \longrightarrow \mathcal{Z}\left(\text { slits } ; \mathbb{R}^{n-1}\right) \quad \text { and } \quad \Xi: \mathbb{R}^{2(g-1)(n-1)(n-2)} \longrightarrow \mathcal{Z}\left(\text { slits; } \mathbb{R}^{n-1}\right) .
$$

The first map $\partial: \mathcal{Z}\left(\lambda\right.$, slits; $\left.\widehat{\mathbb{R}}^{n-1}\right) \rightarrow \mathcal{Z}\left(\right.$ slits; $\left.\mathbb{R}^{n-1}\right)$ is the usual boundary map of $\S 4.4$, and associates with a relative cycle $\sigma \in \mathcal{Z}\left(\lambda\right.$, slits; $\left.\widehat{\mathbb{R}}^{n-1}\right)$ the restriction

$$
\partial \sigma:\{\text { positive slits of } \hat{\lambda}\}=\{\text { slits of } \lambda\} \longrightarrow \mathbb{R}^{n-1}
$$

of its boundary $\partial \sigma$ to positive slits of the orientation cover $\hat{\lambda}$. (Recall that this restriction completely determines $\partial \sigma$ by the definition of twisted relative tangent cycles, since $\partial \sigma_{a}\left(s^{-}\right)=-\partial \sigma_{n-a}\left(s^{+}\right)$when the negative slit $s^{-}$of $\hat{\lambda}$ projects to the same slit of $\lambda$ as the positive slit $s^{+}$.)

The second map $\Xi: \mathbb{R}^{2(g-1)(n-1)(n-2)} \rightarrow Z\left(\right.$ slits; $\left.\mathbb{R}^{n-1}\right)$ associates with each rotationinvariant triangle data function $\tau \in \mathbb{R}^{2(g-1)(n-1)(n-2)}$ the function $\xi^{\tau}$ : slits of $\left.\lambda\right\} \rightarrow \mathbb{R}^{n-1}$ defined by the property that

$$
\xi_{a}^{\tau}(s)=\sum_{b+c=n-a} \tau_{a b c}(s) \in \mathbb{R}
$$


for every slit $s$ of $\lambda$ and every $a=1,2, \ldots, n-1$.

Then the subspace $\mathcal{L}$ consists of all pairs $(\tau, \sigma) \in \mathbb{R}^{2(g-1)(n-1)(n-2)} \times \mathcal{Z}\left(\lambda\right.$, slits; $\left.\widehat{\mathbb{R}}^{n-1}\right)$ such that $\partial \sigma=\Xi(\tau)$ in $\mathcal{Z}\left(\right.$ slits; $\left.\mathbb{R}^{n-1}\right)$.

LEMMA 8.3. The image of the boundary map $\partial: \mathcal{Z}\left(\lambda\right.$, slits; $\left.\widehat{\mathbb{R}}^{n-1}\right) \rightarrow Z\left(\right.$ slits; $\left.\mathbb{R}^{n-1}\right)$ consists of all $\xi \in \mathcal{Z}\left(\right.$ slits; $\left.\mathbb{R}^{n-1}\right)$ such that

$$
\sum_{s \text { slit of } \lambda} \xi_{a}(s)=\sum_{s \text { slit of } \lambda} \xi_{n-a}(s)
$$

for every $a=1,2, \ldots, n-1$. This image has codimension $\left\lfloor\frac{1}{2}(n-1)\right\rfloor$ in $Z\left(\right.$ slits $\left.; \mathbb{R}^{n-1}\right) \cong$ $\mathbb{R}^{12(g-1)(n-1)}$.

Proof. This is an immediate consequence of the homological interpretation of twisted relative tangent cycles in $\S 4.6$, and more precisely of the isomorphism $z\left(\lambda, \operatorname{slits} ; \widehat{\mathbb{R}}^{n-1}\right) \cong$ $H_{1}\left(U, \partial_{\mathrm{v}} U ; \widetilde{\mathbb{R}}^{n-1}\right)$ constructed there.

This construction is well behaved with respect to the boundary maps $\partial$ in the following sense. There is a unique isomorphism $z\left(\operatorname{slits} ; \mathbb{R}^{n-1}\right) \cong H_{0}\left(\partial_{\mathrm{v}} U ; \widetilde{\mathbb{R}}^{n-1}\right)$ defined as follows: this isomorphism associates with $\xi \in \mathcal{Z}\left(\right.$ slits $\left.; \mathbb{R}^{n-1}\right)$ the element of $H_{0}\left(\partial_{\mathrm{v}} U ; \widetilde{\mathbb{R}}^{n-1}\right) \subset$ $H_{0}\left(\partial_{\mathrm{v}} \widehat{U} ; \mathbb{R}^{n-1}\right)$ that assigns to each component of $\partial_{\mathrm{v}} \widehat{U}$ facing a positive slit $s^{+}$the weight $\xi(s) \in \mathbb{R}^{n-1}$ associated by $\xi$ with the projection $s$ of $s^{+}$(and, by definition of the twisting of the coefficient bundle $\widetilde{\mathbb{R}}^{n}$, assigns to the component of $\partial_{\mathrm{v}} \widehat{U}$ facing a negative slit $s^{-}$projecting to $s$ the vector $-\bar{\xi}(s) \in \mathbb{R}^{n-1}$ whose $a$ th coordinate is $\left.-\bar{\xi}_{a}(s)=-\xi_{n-a}(s)\right)$. Then, for these isomorphisms $z\left(\lambda\right.$, slits; $\left.\widehat{\mathbb{R}}^{n-1}\right) \cong H_{1}\left(U, \partial_{\mathrm{v}} U ; \widetilde{\mathbb{R}}^{n-1}\right)$ and $z\left(\right.$ slits; $\left.\mathbb{R}^{n-1}\right) \cong$ $H_{0}\left(\partial_{\mathrm{v}} U ; \widetilde{\mathbb{R}}^{n-1}\right)$, the boundary homomorphism $\partial: \mathcal{Z}\left(\lambda\right.$, slits; $\left.\widehat{\mathbb{R}}^{n-1}\right) \rightarrow \mathcal{Z}\left(\right.$ slits; $\left.\mathbb{R}^{n-1}\right)$ corresponds to the homological boundary $\partial: H_{1}\left(U, \partial_{\mathrm{v}} U ; \widetilde{\mathbb{R}}^{n-1}\right) \rightarrow H_{0}\left(\partial_{\mathrm{v}} U ; \widetilde{\mathbb{R}}^{n-1}\right)$.

Lemma 8.3 is then an immediate consequence of the long exact sequence

$$
\ldots \longrightarrow H_{1}\left(U, \partial_{\mathrm{v}} U ; \widetilde{\mathbb{R}}^{n-1}\right) \longrightarrow H_{0}\left(\partial_{\mathrm{v}} U ; \widetilde{\mathbb{R}}^{n-1}\right) \longrightarrow H_{0}\left(U ; \widetilde{\mathbb{R}}^{n-1}\right) \longrightarrow H_{0}\left(U, \partial_{\mathrm{v}} U ; \widetilde{\mathbb{R}}^{n-1}\right)
$$

using the properties that, because $\widehat{U}$ is connected and $\partial_{\mathrm{v}} \widehat{U}$ is non-empty,

$$
\operatorname{dim} H_{0}\left(U ; \widetilde{\mathbb{R}}^{n-1}\right)=\left\lfloor\frac{1}{2}(n-1)\right\rfloor
$$

and $H_{0}\left(U, \partial_{\mathrm{v}} U ; \widetilde{\mathbb{R}}^{n-1}\right)=0$.

LEMMA 8.4. For $n>3$, the image of $\Xi: \mathbb{R}^{2(g-1)(n-1)(n-2)} \rightarrow Z\left(\right.$ slits $\left.; \mathbb{R}^{n-1}\right)$ consists of all $\xi \in Z$ (slits; $\left.\mathbb{R}^{n-1}\right)$ such that

$$
\xi_{n-1}\left(s_{1}\right)=0 \quad \text { and } \quad \xi_{1}\left(s_{1}\right)=\sum_{a=2}^{n-2}\left(\frac{a-1}{n-3}-1\right) \xi_{a}\left(s_{1}\right)+\sum_{a=2}^{n-2} \frac{a-1}{n-3} \xi_{a}\left(s_{2}\right)+\sum_{a=2}^{n-2} \frac{a-1}{n-3} \xi_{a}\left(s_{3}\right),
$$


whenever $s_{1}, s_{2}$ and $s_{3}$ are the three spikes of the same component $T$ of $S \backslash \lambda$. In particular, the image of $\Xi$ has dimension $12(g-1)(n-3)$.

When $n=3$, the image of $\Xi: \mathbb{R}^{4(g-1)} \rightarrow \mathcal{Z}\left(\right.$ slits $\left.; \mathbb{R}^{2}\right)$ consists of all $\xi \in \mathcal{Z}\left(\right.$ slits $\left.; \mathbb{R}^{2}\right)$ such that

$$
\xi_{2}\left(s_{1}\right)=0 \quad \text { and } \quad \xi_{1}\left(s_{1}\right)=\xi_{1}\left(s_{2}\right)=\xi_{1}\left(s_{3}\right),
$$

whenever $s_{1}, s_{2}$ and $s_{3}$ are the three spikes of the same component $T$ of $S \backslash \lambda$. In particular, the image of $\Xi$ then has dimension $4(g-1)$.

When $n=2$, the image of $\Xi: \mathbb{R}^{0} \rightarrow Z($ slits $; \mathbb{R})$ is of course trivial.

Proof. If $\xi^{\tau}=\Xi(\tau)$ for a rotation-invariant function $\tau \in \mathbb{R}^{2(g-1)(n-1)(n-2)}$, then by definition $\xi_{n-1}^{\tau}(s)=\sum_{b+c=1} \tau_{(n-1) b c}(s)=0$ for every slit $s$, since all indices $b$ and $c$ are supposed to be at least 1 .

Less trivially, if $n>3$ and if $s_{1}, s_{2}$ and $s_{3}$ are the three spikes of a same component $T$ of $S \backslash \lambda$, in this order counterclockwise around $T$,

$$
\begin{aligned}
& \sum_{a=2}^{n-2} \frac{a-1}{n-3} \xi_{a}^{\tau}\left(s_{1}\right)+\sum_{a=2}^{n-2} \frac{a-1}{n-3} \xi_{a}^{\tau}\left(s_{2}\right)+\sum_{a=2}^{n-2} \frac{a-1}{n-3} \xi_{a}^{\tau}\left(s_{3}\right) \\
& \quad=\sum_{a=1}^{n-2} \frac{a-1}{n-3} \sum_{b+c=n-a} \tau_{a b c}\left(s_{1}\right)+\sum_{a=1}^{n-2} \frac{a-1}{n-3} \sum_{b+c=n-a} \tau_{a b c}\left(s_{2}\right)+\sum_{a=1}^{n-2} \frac{a-1}{n-3} \sum_{b+c=n-a} \tau_{a b c}\left(s_{3}\right) \\
& \quad=\sum_{a=1}^{n-2} \frac{a-1}{n-3} \sum_{b+c=n-a} \tau_{a b c}\left(s_{1}\right)+\sum_{b=1}^{n-2} \frac{b-1}{n-3} \sum_{a+c=n-b} \tau_{a b c}\left(s_{1}\right)+\sum_{c=1}^{n-2} \frac{c-1}{n-3} \sum_{a+b=n-c} \tau_{a b c}\left(s_{1}\right) \\
& =\sum_{a, b, c}\left(\frac{a-1}{n-3}+\frac{b-1}{n-3}+\frac{c-1}{n-3}\right) \tau_{a b c}\left(s_{1}\right) \\
& =\sum_{a, b, c} \tau_{a b c}\left(s_{1}\right) \\
& \quad=\sum_{a=1}^{n-1} \xi_{a}^{\tau}\left(s_{1}\right),
\end{aligned}
$$

where the second equality uses the rotation invariance of $\tau$. It follows that

$$
\xi_{1}^{\tau}\left(s_{1}\right)=\sum_{a=2}^{n-2}\left(\frac{a-1}{n-3}-1\right) \xi_{a}^{\tau}\left(s_{1}\right)+\sum_{a=2}^{n-2} \frac{a-1}{n-3} \xi_{a}^{\tau}\left(s_{2}\right)+\sum_{a=2}^{n-2} \frac{a-1}{n-3} \xi_{a}^{\tau}\left(s_{3}\right)
$$

As a consequence, any function $\xi=\Xi(\tau)$ in the image of $\Xi$ satisfies the relations of Lemma 8.4.

Conversely, as a ranges from 2 to $n-2$ and $s$ ranges over all slits of $\lambda$, the functions $\tau \mapsto \xi_{a}^{\tau}(s)$ are linearly independent in the space $\mathbb{R}^{2(g-1)(n-1)(n-2)}$ of rotation-invariant 
triangle data functions $\tau$. Indeed, this follows from a simple computation focusing on the coefficients of the terms $\tau_{1 b c}(s)$ and $\tau_{2 b c}(s)$ in any linear relation between these functions.

The dimension computation then follows from the fact that $\lambda$ has $12(g-1)$ slits. This completes the proof of Lemma 8.4 in the case considered, when $n>3$.

The proof is much simpler when $n=3$, as the triangle data function $\tau$ assigns only one number $\tau_{111}(s)$ to each slit $s$. This makes the argument in this case completely straightforward.

LEMma 8.5. The intersection $\operatorname{im}(\partial) \cap \operatorname{im}(\Xi)$ of the images $\operatorname{im}(\partial)=\partial\left(Z\left(\lambda\right.\right.$, slits; $\left.\left.\widehat{\mathbb{R}}^{n-1}\right)\right)$ and $\operatorname{im}(\Xi)=\Xi\left(\mathbb{R}^{2(g-1)(n-1)(n-2)}\right)$ has dimension

$$
\begin{cases}12(g-1)(n-3)-\left\lfloor\frac{1}{2}(n-1)\right\rfloor, & \text { if } n>3, \\ 4 g-5, & \text { if } n=3 .\end{cases}
$$

As in Lemma 8.4, the case $n=2$ is trivial since $\operatorname{im}(\Xi)=0$ in this case.

Proof. This is an immediate consequence of the characterization of these images in Lemmas 8.3 and 8.4. Indeed, one very easily checks that the restrictions of the $\left\lfloor\frac{1}{2}(n-1)\right\rfloor$ relations of Lemma 8.3 to the image $\operatorname{im}(\Xi)$ are linearly independent.

We now return to the subspace $\mathcal{L} \subset \mathbb{R}^{2(g-1)(n-1)(n-2)} \times \mathcal{Z}\left(\lambda\right.$, slits; $\left.\widehat{\mathbb{R}}^{n-1}\right)$, consisting of all pairs $(\tau, \sigma)$ such that $\partial \sigma=\Xi(\tau)$ in $Z\left(\operatorname{slits} ; \mathbb{R}^{n-1}\right)$. The maps $\Xi$ and $\partial$ induce the same linear map $\mathcal{L} \rightarrow \mathcal{Z}\left(\right.$ slits; $\left.\mathbb{R}^{n-1}\right)$, associating $\Xi(\tau)=\partial \sigma$ with each $(\tau, \sigma) \in \mathcal{L}$. The image of this map is $\operatorname{im}(\partial) \cap \operatorname{im}(\Xi)$, and its kernel is the direct sum of ker $\Xi$ and ker $\partial$. Note that ker $\partial$ is just the space $z\left(\lambda ; \widehat{\mathbb{R}}^{n-1}\right)$ of closed tangent cycles. Therefore, by combining Lemma 8.5, Lemma 8.4 and Proposition 4.7,

$$
\begin{aligned}
\operatorname{dim} \mathcal{L}= & \operatorname{dimim}(\partial) \cap \operatorname{im}(\Xi)+\operatorname{dim} \operatorname{ker} \Xi+\operatorname{dim} \operatorname{ker} \partial \\
= & 12(g-1)(n-3)-\left\lfloor\frac{1}{2}(n-1)\right\rfloor+2(g-1)(n-1)(n-2) \\
& \quad-12(g-1)(n-3)+6(g-1)(n-1)+\left\lfloor\frac{1}{2}(n-1)\right\rfloor \\
= & 2(g-1)\left(n^{2}-1\right)
\end{aligned}
$$

when $n>3$.

When $n=3$, the same argument gives that

$$
\operatorname{dim} \mathcal{L}=(4 g-5)+0+(12 g-11)=16(g-1),
$$

which is equal to $2(g-1)\left(n^{2}-1\right)$ in this case as well.

Since $\mathcal{P}$ is an open convex polyhedral cone in the space $\mathcal{L}$, this concludes the proof of Proposition 8.2. 
Corollary 8.6. The map $\Phi: \operatorname{Hit}_{n}(S) \rightarrow \mathcal{P}$ is a local homeomorphism.

Proof. The map $\Phi$ is continuous by Lemma 8.1, and injective by Corollary 6.8 . By the invariance of the domain theorem, it is therefore a local homeomorphism, since $\operatorname{Hit}_{n}(S)$ and $\mathcal{P}$ have the same dimension by Proposition 8.2.

\subsection{An estimate from the positive intersection condition}

This section is devoted to a key estimate (Lemma 8.8) which will play a crucial role in our proof, completed in the next section $\S 8.3$, that the above map $\Phi: \operatorname{Hit}_{n}(S) \rightarrow \mathcal{P}$ is a global homeomorphism.

For the preimage $\tilde{\lambda}$ of the maximal geodesic lamination $\lambda$ in the universal cover $\widetilde{S}$ of $S$, we want to quantify the complexity of the relative position with respect to each other of the components $T$ of the complement $\widetilde{S} \backslash \tilde{\lambda}$. For this, we choose a train-track neighborhood $U$ of $\lambda$, with preimage $\widetilde{U}$ in $\widetilde{S}$.

We also select an oriented arc $\tilde{k}$ tightly transverse to $\tilde{\lambda}$ in $\widetilde{S}$; recall that this means that $\tilde{k}$ is transverse to the leaves of $\tilde{\lambda}$ and that, for each component $T$ of $\tilde{S} \backslash \tilde{\lambda}$, the intersection $T \cap \tilde{k}$ is either empty, or an arc containing an endpoint of $\tilde{k}$, or an arc joining two distinct components of $\partial T$. As in $\S 4.7$, using Proposition 4.1 , we can arrange by a homotopy respecting $\tilde{\lambda}$ that $\tilde{k}$ is contained in $\widetilde{U}$.

Let $T$ be a component of $\widetilde{S} \backslash \tilde{\lambda}$ that meets $\tilde{k}$, and does not contain any of the endpoints of $\tilde{k}$. Then $\tilde{k} \cap T$ consists of a single arc, since $\tilde{k}$ is tightly transverse to $\tilde{\lambda}$, and $\tilde{k} \cap T$ can be joined to the complement $T \backslash \widetilde{U}$ by a path contained in $T$. We define the divergence radius $r(T) \geqslant 1$ of $T$ with respect to $\widetilde{U}$ and $\tilde{k}$ as the minimum number of branches of $\widetilde{U}$ that are met by a path joining $\tilde{k} \cap T$ to the complement $T \backslash \widetilde{U}$ in $T$.

Lemma 8.7. For every integer $r_{0}$, the number of triangles $T$ with divergence radius $r(T)=r_{0}$ is uniformly bounded, independently of $r_{0}$.

Proof. Instead of counting the components $T$ of $\widetilde{S} \backslash \tilde{\lambda}$ meeting $\tilde{k}$, it is easier to count the components of $\tilde{k} \backslash \tilde{\lambda}$. Cutting $\tilde{k}$ into smaller arcs if necessary, we may assume without loss of generality that $\tilde{k}$ is sufficiently short that it projects to an arc $k$ embedded in $S$. Then there is a natural correspondence between the components of $\tilde{k} \backslash \tilde{\lambda}$ and those of $k \backslash \lambda$. For each component $d$ of $k \backslash \lambda$, let $T_{d}$ be the component of $\widetilde{S} \backslash \tilde{\lambda}$ that contains the component of $\tilde{k} \backslash \tilde{\lambda}$ corresponding to $d$, and define $r(d)=r\left(T_{d}\right)$. We need to show that the number of components $d$ of $k \backslash \lambda$ with $r(d)=r_{0}$ is uniformly bounded.

As $e$ ranges over all branches of the train-track neighborhood $U$, the components of $e \backslash \lambda$ form a family of rectangles $R_{i}$ whose union is equal to $U \backslash \lambda$. In particular, this 
decomposes $U \backslash \lambda$ in two pieces:

(1) the union of the finitely many rectangles $R_{i}$ that meet the boundary $\partial U$;

(2) $12(g-1)$ infinite chains of rectangles $R_{i_{1}} \cup R_{i_{2}} \cup \ldots \cup R_{i_{k}} \cup \ldots$, where each $R_{i_{k}}$ shares with $R_{i_{k+1}}$ a side contained in a tie of $U$, that form the spikes of $U \backslash \lambda$.

Compare Proposition 4.1 and Figure 3.

If $d$ is a component of $k \backslash \lambda$ whose divergence radius $r(d)$ is equal to 1 , then it meets one of the finitely many rectangles $R_{i}$ of (1) above. The number of components of $k \backslash \lambda$ meeting a given rectangle $R_{i}$ is uniformly bounded, by a constant depending on the diameter of the rectangles $R_{i}$ and on the minimum distance between $\tilde{k}$ and its iterates under the action of $\pi_{1}(S)$. Therefore, there are only finitely many components of $k \backslash \lambda$ with divergence radius 1.

If $d$ is a component of $k \backslash \lambda$ with $r(d)>1$, it is contained in one of the spikes $R_{i_{1}} \cup$ $R_{i_{2}} \cup \ldots \cup R_{i_{k}} \cup \ldots$ as in (2) above. In fact, $d$ meets the $(r(d)-1)$-th rectangle $R_{i_{r(d)-1}}$ of this spike, by definition of the divergence radius $r(d)$. Since the number of components of $k \backslash \lambda$ meeting each $R_{i}$ is uniformly bounded, and since there are only $12(g-1)$ spikes, it follows that for $r_{0}>1$ the number of components $d$ of $k \backslash \lambda$ with $r(d)=r_{0}$ is uniformly bounded.

To explain the divergence radius terminology, consider the two sides of $T$ that meet $\tilde{k}$. These two leaves of $\tilde{\lambda}$ follow the same train route in $\widetilde{U}$ over a length of approximately $r(T)$ branches (up to a bounded error term) before diverging at some switch of $\widetilde{U}$.

The side of the oriented arc $\tilde{k}$ where this divergence occurs will greatly matter. There are two possibilities for the two sides of $T$ meeting $\tilde{k}$ : Either they are asymptotic on the left-hand side of $\tilde{k}$, or they are asymptotic on the right-hand side. We say that $T$ points to the left of $\tilde{k}$ in the first case, and points to the right in the second case. We already encountered this terminology in $\S 4.7$ and $\S 6.2$.

Finally, remember that $\hat{\lambda}$ denotes the orientation cover of $\lambda$, and that the covering map $\hat{\lambda} \rightarrow \lambda$ uniquely extends to a cover $\widehat{U} \rightarrow U$ for some train-track neighborhood $\widehat{U}$ of $\hat{\lambda}$.

Let $T_{0}$ be the component of $\widetilde{S} \backslash \tilde{\lambda}$ containing the negative endpoint of $\tilde{k}$. Using the point of view of $\S 4.7$, a relative tangent cycle $\sigma \in \mathcal{Z}(\hat{\lambda}$, slits; $\mathbb{R})$ associates a number $\sigma\left(T_{0}, T\right) \in \mathbb{R}$ with each component $T$ of $\widetilde{S} \backslash \tilde{\lambda}$.

Lemma 8.8. Suppose that the relative tangent cycle $\sigma \in \mathcal{Z}(\hat{\lambda}$, slits; $\mathbb{R}) \cong H_{1}\left(\widehat{U}, \partial_{\mathrm{v}} \widehat{U} ; \mathbb{R}\right)$ satisfies the positive intersection property of $\S 8.1:[\mu] \cdot[\sigma]>0$ for every non-trivial transverse measure $\mu$ for $\hat{\lambda}$, defining a homology class $[\mu] \in H_{1}(\widehat{U} ; \mathbb{R})$. Then, there exists a constant $C>0$ such that, for all but finitely many components $T$ of $\widetilde{S} \backslash \tilde{\lambda}$ meeting $\tilde{k}$,

- $\sigma\left(T_{0}, T\right) \geqslant C r(T)$ if $T$ points to the right of $\tilde{k}$;

- $\sigma\left(T_{0}, T\right) \leqslant-C r(T)$ if $T$ points to the left of $\tilde{k}$. 


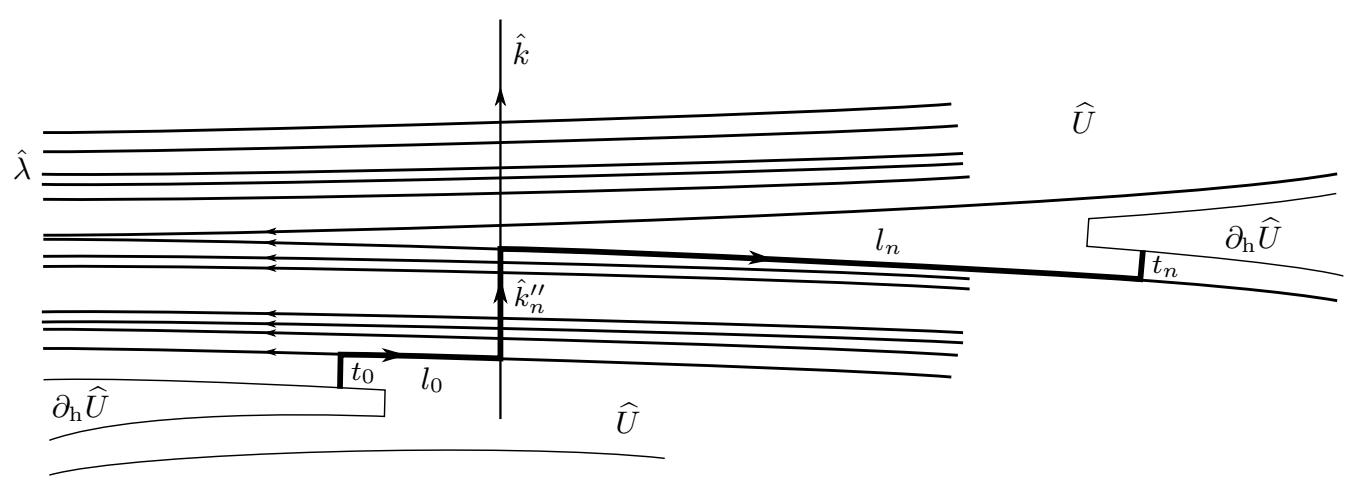

Figure 11. The $\operatorname{arc} \hat{k}_{n}^{\prime}=\hat{k}_{n}^{\prime \prime} \cup l_{0} \cup l_{n} \cup t_{0} \cup t_{n}$.

Proof. Pick a tie $k_{e}$ in each branch $e$ of the train-track neighborhood $\widehat{U}$. Then, for each transverse measure $\mu$ for $\hat{\lambda}$, define

$$
\|\mu\|=\sum_{e} \mu\left(k_{e}\right)
$$

where the sum is over all branches $e$ of $\widehat{U}$. This defines a norm $\|\cdot\|$ on the space $\mathcal{M}(\hat{\lambda}) \subset z(\hat{\lambda} ; \mathbb{R})$ of transverse measures for $\hat{\lambda}$. The space of transverse measures of norm 1 is compact for the weak* topology and, since $\sigma$ satisfies the positive intersection condition, there consequently exists a number $\varepsilon>0$ such that $[\mu] \cdot[\sigma] \geqslant \varepsilon$ for every transverse measure $\mu$ with $\|\mu\|=1$. Let us show that the conclusion of the lemma holds for every $C<\varepsilon$.

For this, we use a proof by contradiction. Suppose that the property does not hold. Then, there exists a sequence of distinct components $T_{n}$ of $\widetilde{S} \backslash \tilde{\lambda}$ meeting $\tilde{k}$ such that $\sigma\left(T_{0}, T_{n}\right)<\operatorname{Cr}\left(T_{n}\right)$ if $T_{n}$ points to the right of $\tilde{k}$, and $\sigma\left(T_{0}, T_{n}\right)>-\operatorname{Cr}\left(T_{n}\right)$ if it points to the left. Passing to a subsequence if necessary, we can arrange that either all $T_{n}$ point to the right, or they all point to the left.

Let us focus attention on the case where all $T_{n}$ point to the left, in which case $\sigma\left(T_{0}, T_{n}\right)>-\operatorname{Cr}\left(T_{n}\right)$ for every $n$. The other case will be similar.

Let $\tilde{k}_{n}$ be the subarc of $\tilde{k}$ going from the negative endpoint of $\tilde{k}$ to an arbitrary point of $\tilde{k} \cap T_{n}$. Let $k_{n}$ be the projection of $\tilde{k}_{n} \subset \widetilde{U}$ to $U$. Among the two lifts of $k_{n}$ to the cover $\widehat{U}$ of $U$, let $\hat{k}_{n}$ be the one where the canonical orientation of the leaves of $\hat{\lambda}$ points to the left for the orientation of $\hat{k}_{n}$ coming from the orientation of $\tilde{k}$. (We are here using the fact that $\tilde{k}$ is tightly transverse to $\tilde{\lambda}$.) In particular, $\hat{k}_{n}$ is tightly transverse to $\hat{\lambda}$ in $\widehat{U}$, and $\sigma\left(T_{0}, T\right)=\sigma\left(\hat{k}_{n}\right)$ by the construction of $\S 4.7$.

Let $\left[\hat{k}_{n}\right] \in H_{1}\left(\widehat{U}, \partial_{\mathrm{h}} \widehat{U} ; \mathbb{R}\right)$ be the relative homology class associated with $\hat{k}_{n}$ as in Step 2 of the proof of Proposition 4.5. Namely, $\left[\hat{k}_{n}\right]$ is represented by an arc $\hat{k}_{n}^{\prime} \subset \widehat{U}$ with $\partial \hat{k}_{n}^{\prime} \subset \partial_{\mathrm{h}} \widehat{U}$ that is made up of the following five pieces: the arc $\hat{k}_{n}^{\prime \prime}$ obtained from $\hat{k}_{n}$ by 
removing the two components of $\hat{k}_{n} \backslash \hat{\lambda}$ that contain its endpoints; two arcs $l_{0}$ and $l_{n}$ in the leaves of $\hat{\lambda}$ that contain the endpoints of $\hat{k}_{n}^{\prime \prime}$; two $\operatorname{arcs} t_{0}$ and $t_{n}$ contained in ties of $\widehat{U}$, with one endpoint in the horizontal boundary $\partial_{\mathrm{h}} \widehat{U}$, with the other endpoint in $\hat{\lambda}$, and whose interior is disjoint from $\hat{\lambda}$. We choose the indexing so that $l_{n}$ joins the positive endpoint of $\hat{k}_{n}^{\prime \prime}$ to the negative endpoint of $t_{n}$, and $l_{0}$ joins the positive endpoint of $t_{0}$ to the negative endpoint of $\hat{k}_{n}^{\prime \prime}$. In addition, we can arrange that $t_{0}$ and $l_{0}$ are independent of $n$. See Figure 11.

By Step 2 of the proof of Proposition 4.5, the homology classes $[\sigma] \in H_{1}\left(\widehat{U}, \partial_{\mathrm{v}} \widehat{U} ; \mathbb{R}\right)$ and $\left[\hat{k}_{n}\right]=\left[\hat{k}_{n}^{\prime}\right] \in H_{1}\left(\widehat{U}, \partial_{\mathrm{h}} \widehat{U} ; \mathbb{R}\right)$ are such that

$$
\left[\hat{k}_{n}^{\prime}\right] \cdot[\sigma]=\sigma\left(\hat{k}_{n}\right)=\sigma\left(T_{0}, T_{n}\right) .
$$

By definition of the divergence radius $r\left(T_{n}\right)$, the $\operatorname{arc} l_{n}$ crosses approximately $r\left(T_{n}\right)$ branches of $\widehat{U}$ (counted with multiplicity). Because the triangles $T_{n}$ are all distinct, $r\left(T_{n}\right)$ tends to infinity as $n$ tends to $\infty$ by Lemma 8.7. Passing to a subsequence if necessary, the standard weak* compactness argument provides a non-trivial transverse measure $\mu$ for $\hat{\lambda}$ such that

$$
\int_{k} \mu=\lim _{n \rightarrow \infty} \frac{1}{r\left(T_{n}\right)} \#\left(k \cap l_{n}\right)
$$

for every arc $k$ transverse to $\hat{\lambda}$, where $\#\left(k \cap l_{n}\right)$ denotes the number of points of $k \cap l_{n}$. In addition, $\|\mu\|=1$ by definition of the norm $\|\cdot\|$.

Note that $\hat{k}_{n}^{\prime} \backslash l_{n}$ has uniformly bounded length. In addition, the orientation of $l_{n}$ coming from the orientation of $\hat{k}_{n}^{\prime}$ is opposite to the canonical orientation of the leaf of $\hat{\lambda}$ that contains it. Therefore,

$$
\lim _{n \rightarrow \infty} \frac{1}{r\left(T_{n}\right)}\left[\hat{k}_{n}^{\prime}\right]=-[\mu]
$$

in $H_{1}\left(\widehat{U}, \partial_{\mathrm{h}} \widehat{U} ; \mathbb{R}\right)$. Then, intersecting with the class $[\sigma] \in H_{1}\left(\widehat{U}, \partial_{\mathrm{v}} \widehat{U} ; \mathbb{R}\right)$ represented by $\sigma \in \mathcal{Z}(\hat{\lambda}$, slits; $\mathbb{R})$ gives

$$
[\mu] \cdot[\sigma]=-\lim _{n \rightarrow \infty} \frac{1}{r\left(T_{n}\right)}\left[\hat{k}_{n}^{\prime}\right] \cdot[\sigma]=-\lim _{n \rightarrow \infty} \frac{1}{r\left(T_{n}\right)} \sigma\left(T_{0}, T_{n}\right) \leqslant C,
$$

since $\sigma\left(T_{0}, T_{n}\right)>-C r\left(T_{n}\right)$ by hypothesis.

Therefore, we have constructed a transverse measure $\mu$ for $\hat{\lambda}$ such that $[\mu] \cdot[\sigma] \leqslant C$ and $\|\mu\|=1$. But this contradicts our hypothesis that $C<\varepsilon \leqslant[\mu] \cdot[\sigma]$ for any such $\mu$, and provides the contradiction sought when all $T_{n}$ point to the left of $\tilde{k}$.

The argument is similar when all $T_{n}$ point to the right. The only difference is that the transverse measure $\mu$ then constructed represents the homology class

$$
[\mu]=\lim _{n \rightarrow \infty} \frac{1}{r\left(T_{n}\right)}\left[\hat{k}_{n}^{\prime}\right]
$$


in $H_{1}\left(\widehat{U}, \partial_{\mathrm{h}} \widehat{U} ; \mathbb{R}\right)$, because the orientation of $l_{n}$ now coincides with the canonical orientation of the leaf of $\hat{\lambda}$ containing it. Since the inequality $\sigma\left(T_{0}, T_{n}\right)<\operatorname{Cr}\left(T_{n}\right)$ is also reversed, this again provides a transverse measure $\mu$ for $\hat{\lambda}$ such that $[\mu] \cdot[\sigma]<C<\varepsilon$ and $\|\mu\|=1$, concluding the proof in this case as well.

COMPLEMENT 8.9. The conclusion of Lemma 8.8 holds when $\sigma$ is replaced by any $\sigma^{\prime}$ in a small neighborhood of $\sigma$ in the finite-dimensional vector space $\mathcal{Z}(\hat{\lambda}$, slits; $\mathbb{R})$.

Proof. By compactness of the space of transverse measures $\mu$ with $\|\mu\|=1$, we can choose $\varepsilon>0$ so that $[\mu] \cdot\left[\sigma^{\prime}\right] \geqslant \varepsilon$ for every $\sigma^{\prime} \in \mathcal{Z}(\hat{\lambda}$, slits; $\mathbb{R})$ sufficiently close to $\sigma$ and every transverse measure $\mu$ with $\|\mu\|=1$. Then the proof shows that the conclusion of Lemma 8.8 holds for any such $\sigma^{\prime}$ and $C<\varepsilon$.

\subsection{Realization of invariants, and parametrization of $\operatorname{Hit}_{n}(S)$}

At the beginning of $\S 8$, we introduced the map

$$
\Phi: \operatorname{Hit}_{n}(S) \longrightarrow \mathcal{P} \subset \mathbb{R}^{6(g-1)(n-1)(n-2)} \times \mathcal{Z}\left(\lambda, \text { slits; } \widehat{\mathbb{R}}^{n}\right)
$$

that associates its triangle invariants and shearing cycle with a Hitchin character. We showed in $\S 8.1$ that the image of $\Phi$ is contained in the convex polyhedral cone $\mathcal{P}$ defined by the triangle rotation condition, the shearing cycle boundary condition and the positive intersection condition. We also showed in Corollary 8.6 that $\Phi: \operatorname{Hit}_{n}(S) \rightarrow \mathcal{P}$ is a local homeomorphism.

Proposition 8.10. The map $\Phi: \operatorname{Hit}_{n}(S) \rightarrow \mathcal{P}$ is proper.

Proof. We need to prove the following property: Let $\left\{\varrho_{i}\right\}_{i \in \mathbb{N}}$ be a sequence $\operatorname{in}_{\operatorname{Hit}_{n}}(S)$ such that $\left\{\Phi\left(\varrho_{i}\right)\right\}_{i \in \mathbb{N}}=\left\{\left(\tau^{\varrho_{i}}, \sigma^{\varrho_{i}}\right)\right\}_{i \in \mathbb{N}}$ converges to a point $\left(\tau^{\infty}, \sigma^{\infty}\right) \in \mathcal{P}$; then the sequence $\left\{\varrho_{i}\right\}_{i \in \mathbb{N}}$ admits a converging subsequence.

For this, we will revisit our proof that a Hitchin character is determined by its triangle invariants and its shearing cycle, as in $\S 6.2$. In that proof, we showed that the fundamental group $\pi_{1}(S)$ is generated by elements $\gamma$ of the type described in Lemma 6.4, and then proved that

$$
\varrho_{i}(\gamma)=\left(\prod_{T \in \mathcal{T}_{g_{0}\left(\gamma h_{0}\right)}}\left(\Theta_{E_{0} F_{0}}^{\sigma^{e_{i}}\left(T_{0}, T\right)} \circ \widehat{\Sigma}_{T^{\circ}}^{i} \Theta_{E_{0} F_{0}}^{-\sigma^{e_{i}}\left(T_{0}, T\right)}\right)\right)^{-1} \circ \Theta_{E_{0} F_{0}}^{\sigma^{\rho_{i}}\left(T_{0}, \gamma T_{0}\right)} \circ \Phi_{0}^{i} \in \mathrm{PGL}_{n}(\mathbb{R})
$$

with the notation of Lemma 6.7, except that $\widehat{\Sigma}_{T}^{i}$ and $\Phi_{0}^{i}$ were respectively called $\widehat{\Sigma}_{T}^{\prime}$ and $\Phi_{0}$ there. Recall that the terms $\Theta_{E_{0} F_{0}}^{\sigma^{\varrho_{i}}\left(T_{0}, T\right)}$ depend only on the shearing cycles $\sigma^{\varrho_{i}}$, whereas $\widehat{\Sigma}_{T}^{i}$ and $\Phi_{0}^{i}$ depend only on the triangle invariants of $\varrho_{i}$. Also, the expression of 
$\widehat{\Sigma}_{T}^{i}$ given in the proof of Lemma 6.6 involves two cases, according to whether $T$ points to the left or to the right as seen from $T_{0}$, as defined there (or again in $\S 8.2$ ).

Lemma 8.11. There exists a constant $C$, independent of $T$, such that

$$
\left\|\Theta_{E_{0} F_{0}}^{\sigma^{e_{i}}\left(T_{0}, T\right)} \circ \widehat{\Sigma}_{T^{\circ}}^{i} \Theta_{E_{0} F_{0}}^{-\sigma^{\varrho_{i}}\left(T_{0}, T\right)}-\operatorname{Id}_{\mathbb{R}^{n}}\right\| \leqslant C \max _{a} e^{-(n-1) \sigma_{a}^{e_{i}}\left(T_{0}, T\right)},
$$

if $T$ points to the right between $T_{0}$ and $\gamma T_{0}$ (as seen from $T_{0}$ ), and

$$
\left\|\Theta_{E_{0} F_{0}}^{\sigma^{\varrho_{i}}\left(T_{0}, T\right)} \circ \widehat{\Sigma}_{T}^{i}{ }^{\circ} \Theta_{E_{0} F_{0}}^{-\sigma^{\varrho_{i}}\left(T_{0}, T\right)}-\operatorname{Id}_{\mathbb{R}^{n}}\right\| \leqslant C \max _{a} e^{(n-1) \sigma_{a}^{\varrho_{i}}\left(T_{0}, T\right)},
$$

if $T$ points to the left.

Proof. Fix for $\mathbb{R}^{n}$ a basis in which the ath term belongs to the line $E_{0}^{(a)} \cap F_{0}^{(n-a+1)}$. Then, by definition, the matrix of $\Theta_{E_{0} F_{0}}^{\sigma^{e_{i}}\left(T_{0}, T\right)}$ in this basis is diagonal, with diagonal entries $e^{u_{1}}, e^{u_{2}}, \ldots, e^{u_{n}}$, where $u_{1}, u_{2}, \ldots, u_{n}$ are uniquely determined by the properties that $u_{a}-u_{a+1}=\sigma_{a}^{\varrho_{i}}\left(T_{0}, T\right)$ and $\sum_{a=1}^{n} u_{a}=0$.

Consider for instance the case where $T$ points to the left. Then the map $\widehat{\Sigma}_{T}^{i}$ respects the flag $E_{0}$, and acts by the identity on each of the lines $E_{0}^{(a)} / E_{0}^{(a-1)}$. Therefore, in the above basis for $\mathbb{R}^{n}$, the matrix $A$ of $\widehat{\Sigma}_{T}^{i}$ is upper triangular with all diagonal entries equal to 1 .

By construction, the map $\widehat{\Sigma}_{T}^{i}$ is completely determined by, and depends continuously on, the triangle invariants $\tau_{a b c}^{\varrho_{i}}(s)$ associated with the slit $s$ of $\lambda$ that is the projection of the spike of $T$ delimited by the two components of $\partial T$ that separate $T_{0}$ from $\gamma T_{0}$. Since these triangle invariants converge to $\tau_{a b c}^{\infty}(s)$, we conclude that each $a b$-entry $A_{a b}$ of the matrix $A$ is uniformly bounded by a constant $C$. We already observed that $A_{a b}=0$ if $a>b$ and $A_{a a}=1$.

Multiplying matrices, we conclude that for $a<b$ the $a b$-entry of the matrix of

$$
\Theta_{E_{0} F_{0}}^{\sigma^{\varrho_{i}}\left(T_{0}, T\right)} \circ \widehat{\Sigma}_{T^{\circ}}^{i} \Theta_{E_{0} F_{0}}^{-\sigma^{\varrho_{i}}\left(T_{0}, T\right)}-\operatorname{Id}_{\mathbb{R}^{n}}
$$

is equal to $A_{a b} e^{u_{a}-u_{b}}$ and bounded by

$$
\left|A_{a b}\right| e^{u_{a}-u_{b}} \leqslant C e^{u_{a}-u_{b}}=C e^{-\sum_{c=a}^{b-1}\left(u_{c+1}-u_{c}\right)}=C e^{\sum_{c=a}^{b-1} \sigma_{c}^{Q_{i}}\left(T_{0}, T\right)} \leqslant C \max _{c} e^{(n-1) \sigma_{c}^{e_{i}}\left(T_{0}, T\right)} .
$$

The other entries of this matrix are 0 since $A_{a b}=0$ if $a>b$, and since $A_{a a}=1$.

This proves the estimate required when the triangle $T$ points to the left.

The proof is almost identical when $T$ points to the right, except that the matrix $A$ is now lower triangular.

We now use the property that the limit $\left(\tau^{\infty}, \sigma^{\infty}\right) \in \mathbb{R}^{6(g-1)(n-1)(n-2)} \times \mathcal{Z}\left(\lambda\right.$, slits; $\left.\widehat{\mathbb{R}}^{n}\right)$ actually belongs to the polyhedron $\mathcal{P}$, and more precisely the fact that the relative tangent cycle $\sigma^{\infty} \in \mathcal{Z}\left(\lambda\right.$, slits; $\left.\widehat{\mathbb{R}}^{n}\right)$ satisfies the positive intersection condition. 
LEMMA 8.12. For $\gamma \in \pi_{1}(S)$ as above, the $\varrho_{i}(\gamma) \in \operatorname{PSL}_{n}(\mathbb{R})$ are bounded independently of $i$.

Proof. Because $\sigma^{\infty}$ satisfies the positive intersection condition, the combination of Lemma 8.8, Complement 8.9 and Lemma 8.11 provides constants $C, D>0$ such that, in the expression

$$
\varrho_{i}(\gamma)=\left(\prod_{T \in \mathcal{T}_{g_{0}\left(\gamma h_{0}\right)}}\left(\Theta_{E_{0} F_{0}}^{\sigma^{\varrho_{i}}\left(T_{0}, T\right)} \circ \widehat{\Sigma}_{T}^{i} \circ \Theta_{E_{0} F_{0}}^{-\sigma^{\varrho_{i}}\left(T_{0}, T\right)}\right)\right)^{-1} \circ \Theta_{E_{0} F_{0}}^{\sigma^{\varrho_{i}}\left(T_{0}, \gamma T_{0}\right)} \circ \Phi_{0}^{i}
$$

the contribution of each triangle $T$ is such that

$$
\left\|\Theta_{E_{0} F_{0}}^{\sigma^{\varrho_{i}}\left(T_{0}, T\right)} \circ \widehat{\Sigma}_{T}^{i} \circ \Theta_{E_{0} F_{0}}^{-\sigma^{\varrho_{i}}\left(T_{0}, T\right)}-\operatorname{Id}_{\mathbb{R}^{n}}\right\| \leqslant C e^{-D r(T)},
$$

for the divergence radius $r(T)$ defined in $\S 8.2$. In addition, for every integer $r_{0} \geqslant 1$, Lemma 8.7 shows that the number of triangles $T$ such that $r(T)=r_{0}$ is bounded independently of $r_{0}$. It follows that the product

$$
\coprod_{T \in \mathcal{T}_{g_{0}\left(\gamma h_{0}\right)}}\left(\Theta_{E_{0} F_{0}}^{\sigma^{\varrho_{i}}\left(T_{0}, T\right)} \circ \widehat{\Sigma}_{T}^{i} \circ \Theta_{E_{0} F_{0}}^{-\sigma^{\varrho_{i}}\left(T_{0}, T\right)}\right)
$$

converges and is uniformly bounded.

By construction, the remaining terms $\Theta_{E_{0} F_{0}}^{\sigma^{e_{i}}\left(T_{0}, \gamma T_{0}\right)}$ and $\Phi_{0}^{i}$ are completely determined by, and depends continuously on, the triangle and shearing invariants of $\varrho_{i}$. Since these invariants converge, it follows that these two terms are also uniformly bounded.

Lemma 8.12 shows that the sequence $\left(\varrho_{i}(\gamma)\right)_{i \in \mathbb{N}}$ admits a converging subsequence in $\mathrm{PSL}_{n}(\mathbb{R})$. Doing this for all $\gamma$ in the finite set of generators for $\pi_{1}(S)$ provided by Lemma 6.4 , we conclude that the sequence $\left(\varrho_{i}\right)_{i \in \mathbb{N}}$ admits a converging subsequence in $\operatorname{Hit}_{n}(S)$.

Therefore, every sequence of $\operatorname{Hit}_{n}(S)$ whose image under $\Phi$ converges in the polyhedron $\mathcal{P}$ admits a converging subsequence in $\operatorname{Hit}_{n}(S)$. This proves that the map $\Phi: \operatorname{Hit}_{n}(S) \rightarrow \mathcal{P}$ is proper, and concludes the proof of Proposition 8.10.

THEOREM 8.13. The map $\Phi: \operatorname{Hit}_{n}(S) \rightarrow \mathcal{P}$ is a homeomorphism from the Hitchin component $\operatorname{Hit}_{n}(S)$ to the polyhedron $\mathcal{P} \subset \mathbb{R}^{6(g-1)(n-1)(n-2)} \times \mathcal{Z}\left(\lambda\right.$, slits; $\left.\widehat{\mathbb{R}}^{n}\right)$.

Proof. The map $\Phi$ is a local homeomorphism by Corollary 8.6, and proper by Proposition 8.10. Since $\Phi$ is injective by Corollary 6.8 and since the convex polytope $\mathcal{P}$ is connected, this proves that $\Phi$ is a homeomorphism. 
Remark 8.14. The formulas of $\S 6.2$, in particular Lemma 6.7, provide an explicit construction for the inverse map $\Phi^{-1}: \mathcal{P} \rightarrow \operatorname{Hit}_{n}(S)$. The boundedness estimates that we just used in the proof of Lemma 8.12 show that the infinite products involved in these formulas do converge. This immediately proves that this inverse map $\Phi^{-1}$ is real analytic.

It can be shown that the forward map $\Phi$ is also analytic, using the fact [10] that the flag curve $\mathcal{F}_{\varrho}: \partial_{\infty} \widetilde{S} \rightarrow \operatorname{Flag}\left(\mathbb{R}^{n}\right)$ depends real analytically on the homomorphism $\varrho$. However, this is beyond the scope of this article.

\subsection{Constraints among triangle invariants, and on shearing cycles}

The shearing cycle boundary condition does more than connecting the boundary of the shearing cycle $\sigma^{\varrho}$ of a Hitchin character $\varrho \in \operatorname{Hit}_{n}(S)$ to its triangle invariants $\tau_{a b c}^{\varrho}(s)$. It also puts constraints between the triangle invariants themselves, and restricts the twisted relative tangent cycles that can occur as shearing cycles of Hitchin characters. As a complement to Theorem 8.13, this section is devoted to emphasizing these somewhat unexpected phenomena, which we already encountered in Lemmas 8.3 and 8.4.

COROLlary 8.15. A rotation-invariant triangle data function $\tau \in \mathbb{R}^{2(g-1)(n-1)(n-2)}$ is the triangle invariant $\tau^{\varrho}$ of a Hitchin character $\varrho \in \operatorname{Hit}_{n}(S)$ if and only if

$$
\sum_{s \text { slit of } \lambda} \sum_{b+c=n-a} \tau_{a b c}(s)=\sum_{s \text { slit of } \lambda} \sum_{b+c=a} \tau_{(n-a) b c}(s)
$$

for every $a=1,2, \ldots, n-1$.

As a consequence, the triangle invariants of Hitchin characters form a linear subspace of codimension $\left\lfloor\frac{1}{2}(n-1)\right\rfloor$ in the space $\mathbb{R}^{2(g-1)(n-1)(n-2)}$ of all rotation-invariant triangle data functions.

Proof. Theorem 8.13 shows that $\tau$ is the triangle invariant of a Hitchin character if and only if there exists a relative cycle $\sigma \in \mathcal{Z}\left(\lambda\right.$, slits; $\left.\widehat{\mathbb{R}}^{n-1}\right)$ such that the pair $(\tau, \sigma)$ satisfies the shearing boundary condition, and such that $\sigma$ satisfies the positive intersection condition.

The proof of Proposition 8.2, and in particular Lemmas 8.3 and 8.5, takes care of the first constraint. More precisely, with the notation of that proof, there exists $\sigma \in \mathcal{Z}\left(\lambda\right.$, slits; $\left.\widehat{\mathbb{R}}^{n-1}\right)$ such that $(\tau, \sigma)$ satisfies the shearing boundary cycle condition if and only if $\Xi(\tau)$ belongs to the image $\operatorname{im}(\partial)$. Lemma 8.3 shows that this is equivalent to the condition stated in Corollary 8.15, while Lemma 8.5 shows that $\Xi^{-1}(\operatorname{im}(\partial))$ has codimension $\left\lfloor\frac{1}{2}(n-1)\right\rfloor$ in $\mathbb{R}^{2(g-1)(n-1)(n-2)}$.

The only thing left to prove is that the positive intersection condition has no impact on this property. Namely, if there exists $\sigma \in \mathcal{Z}\left(\lambda\right.$, slits; $\left.\widehat{\mathbb{R}}^{n-1}\right)$ such that $(\tau, \sigma)$ satisfies the 
shearing cycle boundary condition, the relative tangent cycle $\sigma$ can be chosen so that, in addition, it satisfies the positive intersection condition.

For this, we will use the existence of a closed twisted tangent cycle $\sigma_{0} \in \mathcal{Z}\left(\lambda ; \widehat{\mathbb{R}}^{n-1}\right)$ that satisfies the positive intersection condition. An easy way to construct such a tangent cycle is to consider the shearing cycle $\sigma_{0}=\sigma^{\varrho_{0}} \in Z\left(\lambda\right.$, slits; $\left.\widehat{\mathbb{R}}^{n-1}\right)$ of a Hitchin character $\varrho_{0} \in$ $\operatorname{Hit}_{2}(S) \subset \operatorname{Hit}_{n}(S)$ coming from a discrete homomorphism $\varrho: \pi_{1}(S) \rightarrow \mathrm{PSL}_{2}(\mathbb{R}) \subset \mathrm{PSL}_{n}(\mathbb{R})$. All triangle invariants $\tau_{a b c}^{\varrho_{0}}(s)$ of such a Hitchin character are equal to zero; the easiest way to see this is to apply Lemma 1.1 and to observe that, for every triangle component of $\widetilde{S} \backslash \tilde{\lambda}$ with vertices $\tilde{s}, \tilde{s}^{\prime}$ and $\tilde{s}^{\prime \prime}$, there is an element of $\mathrm{PGL}_{n}(\mathbb{R})$ coming from an element of $\mathrm{PGL}_{2}(\mathbb{R})$ that fixes the flag $\mathcal{F}_{\varrho_{0}}(\tilde{s}) \in \operatorname{Flag}\left(\mathbb{R}^{n}\right)$ and exchanges $\mathcal{F}_{\varrho_{0}}(\tilde{s})$ and $\mathcal{F}_{\varrho_{0}}(\tilde{s})$. It therefore follows from the shearing cycle boundary condition that $\partial \sigma_{0}=0$, namely that $\sigma_{0}$ is closed. And $\sigma_{0}$ satisfies the positive intersection condition by Corollary 7.13.

If the rotation-invariant triangle data function $\tau \in \mathbb{R}^{2(g-1)(n-1)(n-2)}$ satisfies the conditions of Corollary 8.15, we just showed that there exists $\sigma \in Z\left(\lambda\right.$, slits; $\left.\widehat{\mathbb{R}}^{n-1}\right)$ such that $(\tau, \sigma)$ satisfies the shearing cycle boundary condition. For $c>0$ sufficiently large, $\sigma+c \sigma_{0}$ satisfies the positive intersection condition since this property holds for $\sigma_{0}$ and since the space of transverse measures for $\hat{\lambda}$ is finite-dimensional [25], [35]. In addition, the pair $\left(\tau, \sigma+c \sigma_{0}\right)$ satisfies the shearing cycle boundary condition since $\partial\left(\sigma+c \sigma_{0}\right)=\partial \sigma$, and the triangle rotation condition by choice of $\tau$. As a consequence, Theorem 8.13 provides a Hitchin character $\varrho \in \operatorname{Hit}_{n}(S)$ whose triangle invariant $\tau^{\varrho}$ is $\tau$, and whose shearing cycle $\sigma^{\varrho}$ is equal to $\sigma+c \sigma_{0}$.

There is a similar characterization of the shearing cycles of Hitchin characters.

COROLlary 8.16. Let $n>3$. For a twisted relative tangent cycle $\sigma \in \mathcal{Z}\left(\lambda\right.$, slits; $\left.\widehat{\mathbb{R}}^{n-1}\right)$ and for $a=1,2, \ldots, n-1$, let $\partial \sigma_{a}$ be the a-th component of its boundary $\partial \sigma:\{$ slits of $\hat{\lambda}\} \rightarrow$ $\mathbb{R}^{n-1}$. Then, $\sigma$ is the shearing cycle $\sigma^{\varrho}$ of a Hitchin character $\varrho \in \operatorname{Hit}_{n}(S)$ if and only if $\sigma$ satisfies the positive intersection condition and

$$
\begin{aligned}
\partial \sigma_{n-1}\left(s_{1}^{+}\right) & =0 \\
\partial \sigma_{1}\left(s_{1}^{+}\right) & =\sum_{a=2}^{n-2}\left(\frac{a-1}{n-3}-1\right) \partial \sigma_{a}\left(s_{1}^{+}\right)+\sum_{a=2}^{n-2} \frac{a-1}{n-3} \partial \sigma_{a}\left(s_{2}^{+}\right)+\sum_{a=2}^{n-2} \frac{a-1}{n-3} \partial \sigma_{a}\left(s_{3}^{+}\right),
\end{aligned}
$$

whenever $s_{1}^{+}, s_{2}^{+}$and $s_{3}^{+}$are positive slits of the orientation cover $\hat{\lambda}$ that project to the three spikes of the same component $T$ of $S \backslash \lambda$.

As a consequence, the shearing cycles of Hitchin characters form an open convex polyhedral cone in a linear subspace of codimension $24(g-1)$ of

$$
Z\left(\lambda, \text { slits; } \widehat{\mathbb{R}}^{n-1}\right) \cong \mathbb{R}^{18(g-1)(n-1)} .
$$


Proof. This is a consequence of Lemma 8.4 and Proposition 4.7.

CoROLlary 8.17. When $n=3$, a twisted relative tangent cycle $\sigma \in \mathcal{Z}\left(\lambda\right.$, slits; $\left.\widehat{\mathbb{R}}^{2}\right)$ is the shearing cycle $\sigma^{\varrho}$ of a Hitchin character $\varrho \in \operatorname{Hit}_{3}(S)$ if and only if $\sigma$ satisfies the positive intersection condition and

$$
\partial \sigma_{2}\left(s_{1}^{+}\right)=0 \quad \text { and } \quad \partial \sigma_{1}\left(s_{1}^{+}\right)=\partial \sigma_{1}\left(s_{2}^{+}\right)=\partial \sigma_{1}\left(s_{3}^{+}\right)=0,
$$

whenever $s_{1}^{+}, s_{2}^{+}$and $s_{3}^{+}$are positive slits of the orientation cover $\hat{\lambda}$ that project to the three spikes of the same component $T$ of $S \backslash \lambda$. As a consequence, the shearing cycles of Hitchin characters form an open convex polyhedral cone in a subspace of codimension $20(g-1)$ of $z\left(\lambda\right.$, slits; $\left.\widehat{\mathbb{R}}^{2}\right) \cong \mathbb{R}^{36(g-1)}$.

When $n=2$, a twisted relative tangent cycle $\sigma \in \mathcal{Z}(\lambda$, slits; $\widehat{\mathbb{R}})=\mathcal{Z}(\lambda$, slits; $\mathbb{R})$ is the shearing cycle $\sigma^{\varrho}$ of a Hitchin character $\varrho \in \operatorname{Hit}_{2}(S)$ if and only if $\sigma$ is closed (namely $\sigma \in$ $Z(\lambda ; \mathbb{R}))$ and satisfies the positive intersection condition. The shearing cycles of Hitchin characters (=fuchsian representations) consequently form an open convex polyhedral cone in a subspace of codimension $12(g-1)$ of $Z(\lambda$, slits; $\mathbb{R}) \cong \mathbb{R}^{18(g-1)}$.

Proof. This also follows from Lemma 8.4 and Proposition 4.7.

We conclude this article by giving, in the next two sections, two brief applications of the machinery developed in this article. In particular, these applications require the full generality of geodesic laminations (as opposed to the much simpler case of geodesic laminations with finitely many leaves considered in [9]).

\section{The action of pseudo-Anosov homeomorphisms on the Hitchin component}

Let $\varphi: S \rightarrow S$ be a pseudo-Anosov homeomorphism of the surface $S$. We can use our parametrization of $\operatorname{Hit}_{n}(S)$ to show that the action of $\varphi$ on the Hitchin component $\operatorname{Hit}_{n}(S)$ is concentrated in a relatively small factor of $\operatorname{Hit}_{n}(S)$, where it acts linearly. This section is only intended as an illustration of the possible applications of the main results of the article; we are consequently limiting its scope to avoid making an already long article much longer.

The pseudo-Anosov property of $\varphi$ is usually expressed in terms of transverse measured foliations on the surface $S$ [40], [17]. It will be more convenient to use the point of view of [14], so that the homeomorphism $\varphi: S \rightarrow S$ is (isotopic to) a pseudo-Anosov homeomorphism if there exist a geodesic lamination $\lambda^{\mathrm{s}}$, a transverse measure $\mu^{\mathrm{s}}$ for $\lambda^{\mathrm{s}}$ and a number $R>1$ such that, after an isotopy of $\varphi$, the following conditions hold:

(1) each component of the complement of the topological support $\lambda^{\mathrm{s}}$ of $\mu^{\mathrm{s}}$ is a topological disk; 
(2) $\varphi\left(\lambda^{\mathrm{s}}\right)=\lambda^{\mathrm{s}}$;

(3) the pull back $\varphi^{*}\left(\mu^{\mathrm{s}}\right)$ of the transverse measure $\mu^{\mathrm{s}}$ is equal to $R \mu^{\mathrm{s}}$.

The homomorphism $\varphi: S \rightarrow S$ acts on the character variety $X_{\mathrm{PSL}_{n}(\mathbb{R})}(S)$ as $\varrho \mapsto \varrho \circ \varphi_{*}$, where $\varphi_{*}: \pi_{1}(S) \rightarrow \pi_{1}(S)$ is any homomorphism induced by $\varphi$ (by choosing a path joining the base point to its image under $\varphi$ ). When $\varrho \in \mathcal{X}_{\mathrm{PSL}_{n}(\mathbb{R})}(S)$ comes from a Teichmüller character of $\operatorname{Hit}_{2}(S)$, it is immediate that so does $\varrho \circ \varphi_{*}$. By connectedness, it follows that the action $\varrho \mapsto \varrho \circ \varphi_{*}$ respects the Hitchin component $\operatorname{Hit}_{n}(S)$.

Replacing $\varphi$ by one of its powers does not significantly change its dynamics.

Lemma 9.1. There exists an integer $k>0$ and a maximal geodesic lamination $\lambda^{+}$ containing $\lambda^{\mathrm{s}}$ such that $\varphi^{k}\left(\lambda^{+}\right)=\lambda^{+}$after isotopy of $\varphi^{k}$. In addition, $\varphi^{k}$ can be chosen so that it respects each slit of $\lambda^{+}$.

Proof. The homeomorphism $\varphi$ permutes the finitely many slits of $\lambda^{\mathrm{s}}$. Therefore, there exists $k$ such that $\varphi^{k}$ respects each slit.

Let $\lambda^{+}$be any maximal geodesic lamination containing $\lambda^{\mathrm{s}}$. Because each component of $S \backslash \lambda^{\mathrm{s}}$ is a topological disk, or more precisely an ideal polygon, $\lambda^{+}$is obtained from $\lambda^{\mathrm{s}}$ by adding finitely many diagonal leaves joining spikes of these polygons. Since $\varphi^{k}$ respects each slit of $\lambda^{\mathrm{s}}$, namely each spike of $S \backslash \lambda^{\mathrm{s}}$, it can easily be isotoped to respect these diagonal leaves (as well as $\lambda^{\mathrm{s}}$ ). By construction, $\varphi^{k}$ respects each slit of $\lambda^{+}$.

We can now use the maximal geodesic lamination $\lambda^{+}$to construct a parametrization of the Hitchin component $\operatorname{Hit}_{n}(S)$ by the polytope

$$
\mathcal{P} \subset \mathbb{R}^{6(g-1)(n-1)(n-2)} \times \mathcal{Z}\left(\lambda^{+}, \text {slits; } \widehat{\mathbb{R}}^{n-1}\right)
$$

as in Theorem 8.13.

Because $\varphi^{k}$ respects the geodesic lamination $\lambda^{+}$, it acts on $z\left(\lambda^{+}\right.$, slits; $\left.\widehat{\mathbb{R}}^{n-1}\right)$ as follows. Lift $\varphi$ to a homeomorphism $\widetilde{\varphi}: \widetilde{S} \rightarrow \widetilde{S}$ of the universal cover $\widetilde{S}$; in particular, $\widetilde{\varphi}^{k}$ respects the pre-image $\tilde{\lambda}^{+}$of $\lambda^{+}$. Then, using the point of view of $\S 4.7$, define a linear map $\varphi_{\bullet}^{k}: \mathcal{Z}\left(\lambda^{+}\right.$, slits; $\left.\widehat{\mathbb{R}}^{n-1}\right) \rightarrow \mathcal{Z}\left(\lambda^{+}\right.$, slits; $\left.\widehat{\mathbb{R}}^{n-1}\right)$ by the property that $\varphi_{\bullet}^{k}(\alpha)\left(T, T^{\prime}\right)=$ $\alpha\left(\widetilde{\varphi}^{k}(T), \widetilde{\varphi}^{k}\left(T^{\prime}\right)\right)$ for any two components $T$ and $T^{\prime}$ of $\widetilde{S} \backslash \tilde{\lambda}^{+}$.

Proposition 9.2. For the homeomorphism

$$
\Phi: \operatorname{Hit}_{n}(S) \longrightarrow \mathcal{P} \subset \mathbb{R}^{6(g-1)(n-1)(n-2)} \times \mathcal{Z}\left(\lambda^{+}, \text {slits; } \widehat{\mathbb{R}}^{n-1}\right)
$$

provided by Theorem 8.13, the action of $\varphi^{k}$ on $\operatorname{Hit}_{n}(S)$ corresponds to the restriction to $\mathcal{P}$ of the product of the identity $\operatorname{Id}_{\mathbb{R}^{6(g-1)(n-1)(n-2)}}$ and of the linear action of $\varphi^{k}$ on $z\left(\lambda^{+}\right.$, slits; $\left.\widehat{\mathbb{R}}^{n-1}\right)$. 
Proof. For $\varrho \in \operatorname{Hit}_{n}(S)$, we need to compare the triangle invariants $\tau_{a b c}^{\varrho \circ \varphi_{*}^{k}}(s)$ and the shearing cycle $\sigma^{\varrho \circ \varphi_{*}^{k}} \in \mathcal{Z}\left(\lambda^{+}\right.$, slits; $\left.\widehat{\mathbb{R}}^{n-1}\right)$ of $\varrho \circ \varphi_{*}^{k}$ to those of $\varrho$.

Lift $\varphi$ to a homeomorphism $\widetilde{\varphi}: \widetilde{S} \rightarrow \widetilde{S}$ of the universal cover $\widetilde{S}$, which is equivariant with respect to $\varphi_{*}: \pi_{1}(S) \rightarrow \pi_{1}(S)$ in the sense that $\widetilde{\varphi}(\gamma x)=\varphi_{*}(\gamma) \widetilde{\varphi}(x)$ for every $x \in \widetilde{S}$ and $\gamma \in \pi_{1}(S)$. It is well known what $\widetilde{\varphi}$ extends to a homeomorphism of $\widetilde{S} \cap \partial_{\infty} \widetilde{S}$. The flag maps $\mathcal{F}_{\varrho}$ and $\mathcal{F}_{\varrho^{\circ} \varphi_{*}^{k}}: \partial_{\infty} \widetilde{S} \rightarrow \operatorname{Flag}\left(\mathbb{R}^{n}\right)$ are then related by the property that $\mathcal{F}_{\varrho^{\circ} \varphi_{*}^{k}}=\mathcal{F}_{\varrho^{\circ}} \widetilde{\varphi}^{k}$. Going back to the definitions of these invariants and remembering that $\varphi^{k}$ respects each slit of $\lambda^{+}$, it immediately follows that $\varrho$ and $\varrho \circ \varphi_{*}^{k}$ have the same triangle invariants $\tau_{a b c}^{\varrho \circ \varphi_{*}^{k}}(s)=\tau_{a b c}^{\varrho}(s)$, and that $\sigma^{\varrho^{\circ} \varphi_{*}^{k}}=\varphi_{\bullet}^{k}\left(\sigma^{\varrho}\right)$.

This is better described in terms of the map $\pi: \operatorname{Hit}_{n}(S) \rightarrow \mathbb{R}^{6(g-1)(n-1)(n-2)}$ corresponding to the projection of $\operatorname{Hit}_{n}(S) \cong \mathcal{P}$ to the first factor of $\mathbb{R}^{6(g-1)(n-1)(n-2)} \times$ $z\left(\lambda^{+}\right.$, slits; $\left.\widehat{\mathbb{R}}^{n-1}\right)$. Namely, $\pi$ associates its triangle invariants $\tau_{a b c}^{\varrho}(s)$ with a Hitchin character $\varrho \in \operatorname{Hit}_{n}(S)$. The image $\mathcal{L}=\pi\left(\operatorname{Hit}_{n}(S)\right)$ is the vector space of dimension

$$
2(g-1)(n-1)(n-2)-\left\lfloor\frac{1}{2}(n-1)\right\rfloor
$$

determined by Corollary 8.15. This defines a fibration $\pi: \operatorname{Hit}_{n}(S) \rightarrow \mathcal{L}$, where the fiber $\pi^{-1}(\tau)$ above each $\tau \in \mathcal{L}$ is a convex polyhedral cone of dimension

$$
3(g-1)(n-1)+\left\lfloor\frac{1}{2}(n-1)\right\rfloor
$$

in $z\left(\lambda^{+}\right.$, slits; $\left.\widehat{\mathbb{R}}^{n-1}\right) \cong \mathbb{R}^{18(g-1)(n-1)}$.

Then, Proposition 9.2 states that the action of $\varphi^{k}$ on $\operatorname{Hit}_{n}(S)$ respects each fiber $\pi^{-1}(\tau)$, and acts on each of these polyhedral cones $\pi^{-1}(\tau) \subset z\left(\lambda^{+}\right.$, slits; $\left.\widehat{\mathbb{R}}^{n-1}\right)$ by restriction of $\varphi^{k}: z\left(\lambda^{+}\right.$, slits; $\left.\widehat{\mathbb{R}}^{n-1}\right) \rightarrow z\left(\lambda^{+}\right.$, slits; $\left.\widehat{\mathbb{R}}^{n-1}\right)$.

If $U$ is a train-track neighborhood of $\lambda^{+}$, the endomorphism $\varphi^{k}$ of $z\left(\lambda^{+}\right.$, slits; $\left.\widehat{\mathbb{R}}^{n-1}\right) \cong$ $H_{1}\left(U, \partial_{\mathrm{v}} U ; \widetilde{\mathbb{R}}^{n-1}\right)$ can be explicitly described in terms of a classical object associated with the pseudo-Anosov homeomorphism $\varphi$, namely the incidence matrix of $\varphi$ with respect to the train track $U$ (see for instance [17, Exposés 9-10]). However, this would take us beyond the intended scope of this article.

\section{Length functions of measured laminations}

One of the motivations for this article is to extend to the Hitchin component the differential calculus of lengths of simple closed curves that was developed for hyperbolic geometry in [39], [42], [6], [5].

For a Hitchin character $\varrho \in \operatorname{Hit}_{n}(S)$, the length functions $\ell_{1}^{\varrho}, \ell_{2}^{\varrho}, \ldots, \ell_{n-1}^{\varrho}$, of [15] and $\S 7.1$ can be restricted to Thurston's space $\mathcal{M L}(S)$ of measured geodesic laminations. 
There is just a little subtlety, which is that the geodesic currents discussed in $\S 7.1$ form a completion of the set of homotopy classes of oriented closed curves, whereas $\mathcal{M} \mathcal{L}(S)$ completes the set of homotopy classes of unoriented simple closed curves.

An unoriented simple closed curve $\gamma$ in $S$ defines two oriented curves $\gamma^{*}$ and $\gamma^{* *}$, one for each orientation, and therefore two measure geodesic currents that we also denote by $\gamma^{*}, \gamma^{* *} \in \mathrm{e}^{\mathrm{m}}(S)$. Then there is a unique continuous embedding $\iota: \mathcal{M} \mathcal{L}(S) \rightarrow \mathrm{e}^{\mathrm{m}}(S)$ that is homogeneous, in the sense that $\iota(t \mu)=t \iota(\mu)$ for every $\mu \in \mathcal{M} \mathcal{L}(S)$ and every $t>0$, and such that $\iota(\gamma)=\frac{1}{2}\left(\gamma^{*}+\gamma^{* *}\right)$ for every simple closed curve $\gamma \in \mathcal{M} \mathcal{L}(S)$; see for instance [3]. Combining this embedding with $\ell_{a}^{\varrho}: \mathrm{C}^{\mathrm{m}}(S) \rightarrow \mathbb{R}$ defines, for each $a=1,2, \ldots, n-1$, a length function $\ell_{a}^{\varrho}: \mathcal{N} \mathcal{L}(S) \rightarrow \mathbb{R}$. The definition, and in particular the introduction of the factor $\frac{1}{2}$, is designed so that when $n=2$ the function $\ell_{1}^{\varrho}$ coincides with Thurston's length function $\ell^{\varrho}: \mathcal{M} \mathcal{L}(S) \rightarrow \mathbb{R}$ for the hyperbolic metric on $S$ associated with $\varrho \in \operatorname{Hit}_{2}(S)$, which plays a fundamental role in hyperbolic geometry; see for instance [40], [17], [39], [3], [33] for a few applications of this length function $\ell^{\varrho}$.

Because $\ell_{a}^{\varrho}\left(\gamma^{* *}\right)=\ell_{n-a}^{\varrho}\left(\gamma^{*}\right)$, the length functions $\ell_{a}^{\varrho}$ and $\ell_{n-a}^{\varrho}$ coincide on $\mathcal{M} \mathcal{L}(S)$ so that, in practice, we have only $\left\lfloor\frac{1}{2} n\right\rfloor$ length functions $\ell_{a}^{\varrho}: \mathcal{M} \mathcal{L}(S) \rightarrow \mathbb{R}$.

The space $\mathcal{M} \mathcal{L}(S)$ of measured geodesic laminations is homeomorphic to $\mathbb{R}^{6(g-1)}$, but admits no differentiable structure that is respected by the action of the mapping class group. As a consequence, we cannot use the standard concepts of differential calculus on this space.

However, $\mathcal{M} \mathcal{L}(S)$ is naturally endowed with a piecewise integral linear structure; this means that it admits an atlas locally modelling it over $\mathbb{R}^{6(g-1)}$ where the coordinate changes are piecewise linear and where the linear pieces of these coordinate changes have integer coefficients [39], [36]. In particular, because a piecewise linear map does have a tangent map, a consequence of the piecewise linear structure is that $\mathcal{M} \mathcal{L}(S)$ admits a well-defined tangent space $T_{\mu} \mathcal{M} \mathcal{L}(S)$ at each point $\mu \in \mathcal{M} \mathcal{L}(S)$.

Each tangent space $T_{\mu} \mathcal{M} \mathcal{L}(S)$ is homeomorphic to $\mathbb{R}^{6(g-1)}$ and is homogeneous, in the sense that there is a well-defined multiplication of tangent vectors by non-negative numbers, but it is not always a vector space. Indeed, there exist points $\mu \in \mathcal{N} \mathcal{L}(S)$ where the tangent space $T_{\mu} \mathcal{M} \mathcal{L}(S)$ admits no vector space structure which is respected by all coordinate charts; a typical example of such points are the positive real multiples of simple closed curves, which are dense in $\mathcal{M} \mathcal{L}(S)$. Conversely, at a measured geodesic lamination $\mu$ whose support is a maximal geodesic lamination, the piecewise integral linear structure does define a natural vector space structure on the tangent space $T_{\mu} \mathcal{M} \mathcal{L}(S)$; these $\mu$ form a subset of full measure in $\mathcal{M} \mathcal{L}(S)$. See [42], for instance. 
THEOREM 10.1. For a Hitchin character $\varrho \in \operatorname{Hit}_{n}(S)$ and for every $a=1,2, \ldots,\left\lfloor\frac{1}{2} n\right\rfloor$, the length function $\ell_{a}^{\varrho}: \mathcal{M} \mathcal{L}(S) \rightarrow \mathbb{R}$ admits a tangent map $T_{\mu} \ell_{a}^{\varrho}: T_{\mu} \mathcal{M} \mathcal{L}(S) \rightarrow \mathbb{R}$ at each $\mu \in \mathcal{M} \mathcal{L}(S)$, in the following sense. For $\mu \in \mathcal{M} \mathcal{L}(S)$ and $v \in T_{\mu} \mathcal{M} \mathcal{L}(S)$, let $t \mapsto \alpha_{t}$ be a curve in $\mathcal{M} \mathcal{L}(S)$ such that $\alpha_{0}=\mu$ and the right-hand-side tangent derivative

$$
\left.\frac{d}{d t^{+}} \alpha_{t}\right|_{t=0}
$$

exists and is equal to $v$. Then

$$
\left.\frac{d}{d t^{+}} \ell_{a}^{\varrho}\left(\alpha_{t}\right)\right|_{t=0}=T_{\mu} \ell_{a}^{\varrho}(v) \in \mathbb{R} .
$$

Proof. See $[15, \S 3.2]$.

The proof of Theorem 10.1 relies on two key ingredients: the analytic interpretation [7], [6] of tangent vectors $v \in T_{\mu} \mathcal{M} \mathcal{L}(S)$ as a certain type of Hölder geodesic currents as in $\S 7.1$; and the continuity of the length function $\ell_{a}^{\varrho}: \mathrm{e}^{\mathrm{H}}(S) \rightarrow \mathbb{R}$ for the Hölder topology, proved in [15]. In particular, $T_{\mu} \ell_{a}^{\varrho}(v)$ is equal to the ath length $\ell_{a}^{\varrho}(v)$ of the Hölder geodesic current $v \in \mathrm{e}^{\mathrm{H}}(S)$ associated with $v \in T_{\mu} \mathcal{M} \mathcal{L}(S)$.

The results of the current paper, and in particular Theorem 7.5, provide a description of the tangent map $T_{\mu} \ell_{a}^{\varrho}$ on the faces of $T_{\mu} \mathcal{M} \mathcal{L}(S)$.

This is based on a more combinatorial interpretation, also developed in [6] and [7], of tangent vectors $v \in T_{\mu} \mathcal{M} \mathcal{L}(S)$ as tangent cycles for geodesic laminations $\lambda$ containing the support $\lambda_{\mu}$ of $\mu$; these tangent cycles must satisfy a certain positivity condition (unrelated to the positive intersection condition of $\$ 8.1$ ). This decomposes the tangent space $T_{\mu} \mathcal{M} \mathcal{L}(S)$ into a family of cones $F_{\lambda}$, indexed by geodesic laminations $\lambda$ containing the support $\lambda_{\mu}$ of $\mu$, where $F_{\lambda}$ consists of those tangent vectors $v \in T_{\mu} \mathcal{M} \mathcal{L}(S)$ that can be described as tangent cycles for $\lambda$. In particular, each $F_{\lambda}$ is naturally identified to a convex polyhedral cone in the vector space $z(\lambda ; \mathbb{R})$ of all tangent cycles for $\lambda$, and the homogeneous and additive structure induced on the cone $F_{\lambda}$ by the vector space structure of $\mathcal{Z}(\lambda ; \mathbb{R})$ is compatible with the piecewise linear structure of $\mathcal{N} \mathcal{L}(S)$. The $F_{\lambda}$ are the faces of $T_{\mu} \mathcal{M} \mathcal{L}(S)$ for the piecewise linear structure of $\mathcal{M} \mathcal{L}(S)$. See [42] for a slightly different approach.

In the generic case where the support $\lambda_{\mu}$ of $\mu \in \mathcal{M} \mathcal{L}(S)$ is maximal, there is only one face in $T_{\mu} \mathcal{M} \mathcal{L}(S)$, namely $F_{\lambda_{\mu}}$. This face $F_{\lambda_{\mu}}$ is equal to the whole vector space $\mathcal{Z}\left(\lambda_{\mu} ; \mathbb{R}\right)$ of tangent cycles for $\lambda_{\mu}$.

Because of the positivity condition involved in the interpretation of tangent vectors $v \in T_{\mu} \mathcal{M} \mathcal{L}(S)$ as tangent cycles for geodesic laminations, it is quite possible that different geodesic laminations $\lambda$ and $\lambda^{\prime}$ define the same face $F_{\lambda}=F_{\lambda^{\prime}}$. The correspondence $\lambda \mapsto F_{\lambda}$ 
can be made bijective by restricting attention to chain recurrent geodesic laminations [42], [7]. Instead of chain recurrent geodesic laminations, we will focus on the case where the geodesic lamination $\lambda$ is maximal, as it is better adapted to our purposes. Every geodesic lamination $\lambda^{\prime}$ is contained in a maximal geodesic lamination $\lambda$, so that every face of $T_{\mu} \mathcal{M} \mathcal{L}(S)$ is contained in a face $F_{\lambda}$ associated with a maximal geodesic lamination $\lambda$. Note that, although $\lambda$ is maximal, the dimension of the associated face $F_{\lambda}$ may be significantly smaller than the dimension $6(g-1)$ of $T_{\mu} \mathcal{M} \mathcal{L}(S)$.

THEOREM 10.2. The tangent map $T_{\mu} \ell_{a}^{\varrho}: T_{\mu} \mathcal{M} \mathcal{L}(S) \rightarrow \mathbb{R}$ is linear on each face of $T_{\mu} \mathcal{M} \mathcal{L}(S)$.

More precisely, if the face $F_{\lambda} \subset T_{\mu} \mathcal{M} \mathcal{L}(S)$ is associated with a maximal geodesic lamination $\lambda$, if we interpret the tangent vector $v \in F_{\lambda}$ as a tangent cycle for $\lambda$, and if $\sigma^{\varrho} \in Z\left(\lambda\right.$, slits; $\left.\widehat{\mathbb{R}}^{n-1}\right)$ is the shearing cycle of $\varrho$, then

$$
T_{\mu} \ell_{a}^{\varrho}(v)=\left[\sigma_{a}^{\varrho}\right] \cdot[v]
$$

where, as in $\S 4.5$ and $\S 7.2$, the dot $\cdot$ denotes the algebraic intersection number in a traintrack neighborhood $\widehat{U}$ of the orientation cover $\hat{\lambda}$ of $\lambda,\left[\sigma_{a}^{\varrho}\right] \in H_{1}\left(\widehat{U}, \partial_{\mathrm{v}} \widehat{U} ; \mathbb{R}\right)$ is the a-th component of the twisted relative homology class

$$
\left[\sigma^{\varrho}\right] \in H_{1}\left(U, \partial_{\mathrm{v}} U ; \widetilde{\mathbb{R}}^{n-1}\right) \subset H_{1}\left(\widehat{U}, \partial_{\mathrm{v}} \widehat{U} ; \mathbb{R}^{n-1}\right)
$$

defined by $\sigma^{\varrho} \in Z\left(\lambda\right.$, slits; $\left.\widehat{\mathbb{R}}^{n-1}\right)$, and $[v] \in H_{1}(\widehat{U} ; \mathbb{R})$ is the homology class represented by $v \in Z(\lambda ; \mathbb{R}) \subset Z(\hat{\lambda} ; \mathbb{R})$.

Proof. We already observed that $T_{\mu} \ell_{a}^{\varrho}(v)=\ell_{a}^{\varrho}(v)$ where the right-hand side interprets $v$ as a tangent cycle for $\lambda$ and involves the function $\ell_{a}^{\varrho}: Z(\hat{\lambda}) \rightarrow \mathbb{R}$ introduced in $\S 7.1$. The formula then occurs as a special case of Theorem 7.5.

\section{References}

[1] Birman, J. S. \& Series, C., Geodesics with bounded intersection number on surfaces are sparsely distributed. Topology, 24 (1985), 217-225.

[2] Bonahon, F., Bouts des variétés hyperboliques de dimension 3. Ann. of Math., 124 (1986), 71-158.

[3] - The geometry of Teichmüller space via geodesic currents. Invent. Math., 92 (1988), 139-162.

[4] - Geodesic currents on negatively curved groups, in Arboreal Group Theory (Berkeley, CA, 1988), Math. Sci. Res. Inst. Publ., 19, pp. 143-168. Springer, New York, 1991.

[5] - Shearing hyperbolic surfaces, bending pleated surfaces and Thurston's symplectic form. Ann. Fac. Sci. Toulouse Math., 5 (1996), 233-297.

[6] - Geodesic laminations with transverse Hölder distributions. Ann. Sci. École Norm. Sup., 30 (1997), 205-240. 
[7] — Transverse Hölder distributions for geodesic laminations. Topology, 36 (1997), 103-122.

[8] - Geodesic laminations on surfaces, in Laminations and Foliations in Dynamics, Geometry and Topology (Stony Brook, NY, 1998), Contemp. Math., 269, pp. 1-37. Amer. Math. Soc., Providence, RI, 2001.

[9] Bonahon, F. \& Dreyer, G., Parameterizing Hitchin components. Duke Math. J., 163 (2014), 2935-2975.

[10] Bridgeman, M., Canary, R., Labourie, F. \& Sambarino, A., The pressure metric for Anosov representations. Geom. Funct. Anal., 25 (2015), 1089-1179.

[11] Bridson, M. R. \& Haefliger, A., Metric Spaces of Non-Positive Curvature. Grundlehren der Mathematischen Wissenschaften, 319. Springer, Berlin-Heidelberg, 1999.

[12] Calegari, D., Foliations and the Geometry of 3-Manifolds. Oxford Mathematical Monographs. Oxford Univ. Press, Oxford, 2007.

[13] Canary, R. D., Epstein, D. B. A. \& Green, P., Notes on notes of Thurston, in Analytical and Geometric Aspects of Hyperbolic Space (Coventry/Durham, 1984), London Math. Soc. Lecture Note Ser., 111, pp. 3-92. Cambridge Univ. Press, Cambridge, 1987.

[14] Casson, A. J. \& Bleiler, S. A., Automorphisms of Surfaces after Nielsen and Thurston. London Mathematical Society Student Texts, 9. Cambridge Univ. Press, Cambridge, 1988.

[15] Dreyer, G., Length functions of Hitchin representations. Alg. Geom. Top., 13 (2013), 3153-3173.

[16] - Thurston's cataclysm deformations for Anosov representations. Preprint, 2013. arXiv:1301.6961 [math.GT].

[17] Fathi, A., Laudenbach, F. \& Pó́naru, V., Travaux de Thurston sur les surfaces. Astérisque, 66. Société Mathématique de France, Paris, 1979.

[18] Fock, V. V. \& Goncharov, A. B., Moduli spaces of local systems and higher Teichmüller theory. Publ. Math. Inst. Hautes Études Sci., 103 (2006), 1-211.

[19] Ghys, É. \& DE LA HARPe, P. (EDS.), Sur les groupes hyperboliques d'après Mikhael Gromov. Progress in Mathematics, 83. Birkhäuser, Boston, MA, 1990.

[20] Gromov, M., Hyperbolic groups, in Essays in Group Theory, Math. Sci. Res. Inst. Publ., 8, pp. 75-263. Springer, New York, 1987.

[21] Guichard, O., Composantes de Hitchin et représentations hyperconvexes de groupes de surface. J. Differential Geom., 80 (2008), 391-431.

[22] Guichard, O. \& Wienhard, A., Anosov representations: domains of discontinuity and applications. Invent. Math., 190 (2012), 357-438.

[23] Hitchin, N. J., Lie groups and Teichmüller space. Topology, 31 (1992), 449-473.

[24] Katok, A. \& Hasselblatt, B., Introduction to the Modern Theory of Dynamical Systems. Encyclopedia of Mathematics and its Applications, 54. Cambridge Univ. Press, Cambridge, 1995.

[25] Kаток, A. B., Invariant measures of flows on orientable surfaces. Dokl. Akad. Nauk SSSR, 211 (1973), 775-778.

[26] Kenyon, R., A group of paths in $\mathbf{R}^{2}$. Trans. Amer. Math. Soc., 348 (1996), 3155-3172.

[27] Labourie, F., Anosov flows, surface groups and curves in projective space. Invent. Math., 165 (2006), 51-114.

[28] - Flat projective structures on surfaces and cubic holomorphic differentials. Pure Appl. Math. Q., 3 (2007), 1057-1099.

[29] - Cyclic surfaces and Hitchin components in rank 2. Ann. of Math., 185 (2017), 1-58.

[30] Loftin, J. C., Affine spheres and convex $\mathbb{R P}^{n}$-manifolds. Amer. J. Math., 123 (2001), 255-274. 
[31] Lusztig, G., Total positivity in reductive groups, in Lie Theory and Geometry, Progr. Math., 123, pp. 531-568. Birkhäuser Boston, Boston, MA, 1994.

[32] — Total positivity in partial flag manifolds. Represent. Theory, 2 (1998), 70-78.

[33] Mirzakhani, M., Growth of the number of simple closed geodesics on hyperbolic surfaces. Ann. of Math., 168 (2008), 97-125.

[34] Mumford, D., Fogarty, J. \& Kirwan, F., Geometric Invariant Theory. Ergebnisse der Mathematik und ihrer Grenzgebiete, 34. Springer, Berlin-Heidelberg, 1994.

[35] Papadopoulos, A., Deux remarques sur la géométrie symplectique de l'espace des feuilletages mesurés sur une surface. Ann. Inst. Fourier (Grenoble), 36 (1986), 127-141.

[36] Penner, R. C. \& Harer, J. L., Combinatorics of Train Tracks. Annals of Mathematics Studies, 125. Princeton Univ. Press, Princeton, NJ, 1992.

[37] Procesi, C., The invariant theory of $n \times n$ matrices. Advances in Math., 19 (1976), 306381.

[38] Ruelle, D. \& Sullivan, D. P., Currents, flows and diffeomorphisms. Topology, 14 (1975), $319-327$.

[39] Thurston, W. P., The geomety and topology of three-manifolds. Princeton Lecture Notes, 1980. Available at http://library.msri.org/books/gt3m/.

[40] - On the geometry and dynamics of diffeomorphisms of surfaces. Bull. Amer. Math. Soc., 19 (1988), 417-431.

[41] — Three-manifolds, foliations and circles, I. Preprint, 1997. arXiv:math/9712268 [math.GT].

[42] — Minimal stretch maps between hyperbolic surfaces. Preprint, 1998. arXiv:math/9801039 [math.GT] .

Francis Bonahon

Department of Mathematics University of Southern California KAP 108

Los Angeles, CA 90089-2532

U.S.A.

fbonahon@math.usc.edu

Received May 26, 2015

Received in revised form March 19, 2017
Guillaume Dreyer

Department of Mathematics

University of Notre Dame

255 Hurley Hall

Notre Dame, IN 46556

U.S.A.

and

Department of Mathematics University of Southern California

KAP 108

Los Angeles, CA 90089-2532

U.S.A.

dreyfactor@gmail.com 